University of Louisville

ThinkIR: The University of Louisville's Institutional Repository

8-2008

\title{
Principal theories of practice : mapping the cognitive structure and effects of instructional leadership
}

Gary W. Houchens

University of Louisville

Follow this and additional works at: https://ir.library.louisville.edu/etd

\section{Recommended Citation}

Houchens, Gary W., "Principal theories of practice : mapping the cognitive structure and effects of instructional leadership" (2008). Electronic Theses and Dissertations. Paper 639.

https://doi.org/10.18297/etd/639

This Doctoral Dissertation is brought to you for free and open access by ThinkIR: The University of Louisville's Institutional Repository. It has been accepted for inclusion in Electronic Theses and Dissertations by an authorized administrator of ThinkIR: The University of Louisville's Institutional Repository. This title appears here courtesy of the author, who has retained all other copyrights. For more information, please contact thinkir@louisville.edu. 
PRINCIPAL THEORIES OF PRACTICE: MAPPING THE COGNITIVE STRUCTURE AND EFFECTS OF INSTRUCTIONAL LEADERSHIP

\title{
By
}

Gary W. Houchens

B.A., Western Kentucky University, 1993

M.A., Western Kentucky University, 2000

M.A.T., Oakland City University, 2003

\author{
A Dissertation \\ Submitted to the Faculty of the \\ Graduate School of the University of Louisville and the \\ Graduate School of Western Kentucky University \\ in Partial Fulfillment of the Requirements \\ for the Degree of \\ Doctor of Philosophy \\ Department of Educational Leadership, Foundations and Human Resource Education \\ University of Louisville \\ Department of Educational Administration, Leadership, and Research \\ Western Kentucky University
}

August 2008 

Copyright 2008 by Gary W. Houchens

All rights reserved 
PRINCIPAL THEORIES OF PRACTICE: MAPPING THE COGNITIVE STRUCTURE AND EFFECTS OF INSTRUCTIONAL LEADERSHIP

\section{By}

Gary Wayne Houchens

B.A., Western Kentucky University, 1993

M.A., Western Kentucky University, 2000

M.A.T., Oakland City University, 2003

A Dissertation Approved on

May 12, 2008

by the following Dissertation Committee:

Dissertation Director 


\section{DEDICATION}

This dissertation is dedicated to my parents

Wayne and Sandra Houchens

and my wife

Dr. Holly J. Payne 


\section{ACKNOWLEDGEMENTS}

I would like to thank my dissertation director, Dr. John L. Keedy, for guiding, mentoring and encouraging me through the process of planning, researching and writing this dissertation and through two national conference presentations. His advice and support have been invaluable, and I am so happy to bring his vision for exploring principal theories of practice to fruition. I would also like to thank my other committee members, Dr. Paul Winter, Dr. Thaddeus Dumas, Dr. Bud Schlinker, and Dr. Gayle Ecton, for their comments and assistance over the past year. I would like to express my deep thanks and appreciation to my wife, Dr. Holly J. Payne, for her understanding, patience, encouragement, and editing. She has been a constant source of strength since long before this process began. My heart is also full of gratitude and affection for my fellow doctoral students, Winnie Cohron, Dana Cosby Simmons, Janet Hurt, and James McCaslin, who have shared every step of this journey with me. Finally, many thanks to the students and staff of the Learning Opportunities Center Alternative School where I serve as principal. They are the ultimate inspiration for my own reflective practice and for this dissertation. 


\section{ABSTRACT \\ PRINCIPAL THEORIES OF PRACTICE: \\ MAPPING THE COGNITIVE STRUCTURE \& EFFECTS OF INSTRUCTIONAL LEADERSHIP}

Gary W. Houchens

May 12, 2008

This dissertation builds on the work of Argyris and Schön (1974), who explained patterns of organizational learning using a concept called theories of practice, cognitive formulas for professional problem solving. Theories of practice consist of deeply held assumptions that logically imply certain action strategies. Argyris and Schön hypothesized that by engaging in deep reflection on assumptions and action strategies, professionals could develop more effective theories of practice based on alternate assumptions and action strategies. This dissertation explores the instructional leadership theories of practice of four successful school principals using a naturalistic, qualitative, multi-case design. Data gathering methods included interviews, observations, and a written reflective exercise completed by principals. The researcher used constant comparative analysis to categorize data until the theories of practice for each principal emerged. Three research questions framed the study. The first question identified the principals' instructional leadership theories of practice. Findings revealed that these principals used three to six theories of practice based on strong moral and utilitarian assumptions regarding the inherent dignity and worth of both teachers and students, and the positive academic effects of recognizing and affirming that worth. Action strategies 
included building positive relationships, inviting teacher input, fostering collaboration, unifying staff around a common mission, and encouraging continuous teacher professional growth. The second research question investigated the effect of principal theories of practice on teachers. Teachers from the case study schools reported that principal theories of practice affected them in a variety of positive ways, including higher levels of job satisfaction and motivation, strong affiliation with the school, and a sense of personal responsibility for student outcomes. The third research question explored the extent to which principals engaged reflection on their practice. Findings were limited to only two examples: (a) principals engaged in double-loop learning by developing "special case" theories of practice for correcting underperforming teachers who failed to respond to the principals' preferred methods of leadership, and (b) by learning from feedback to place more emphasis on inviting teacher input. The final chapter discusses the study's implications for principal preparation programs, school districts, policy makers, and principal professional development. 


\section{TABLE OF CONTENTS}

PAGE

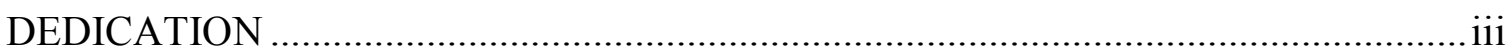

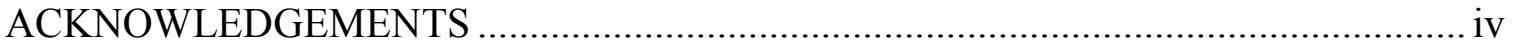

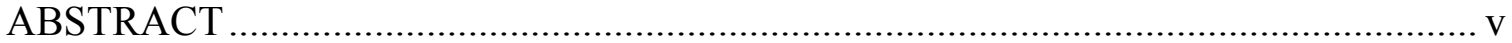

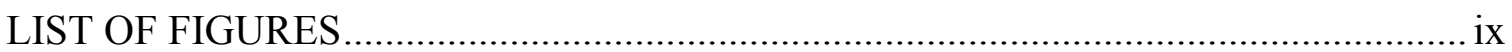

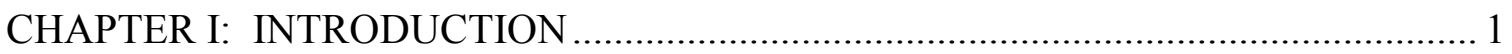

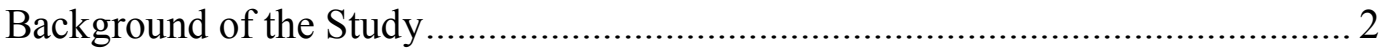

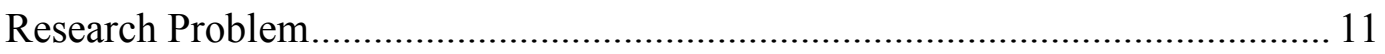

Rationale

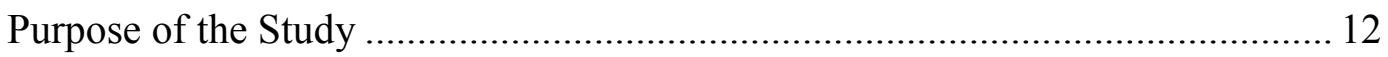

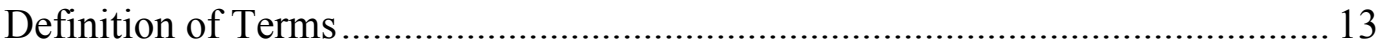

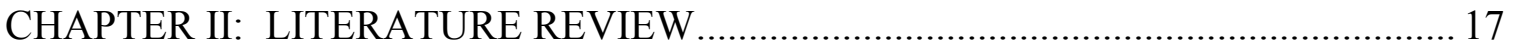

Education Reform: Standardization and Decentralization ................................... 18

Principal Effects and Student Achievement....................................................... 26

Resistance to Change: Confronting the Culture of Schools ................................... 43

Principal Effects on School Culture, Teacher Perceptions, and

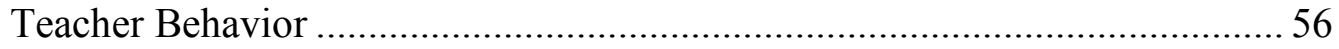

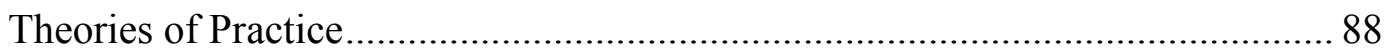

Conceptual Framework for the Study ………………........................................ 107

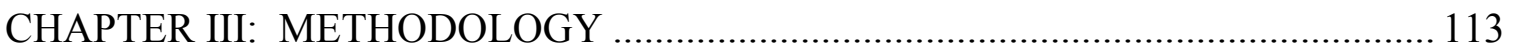

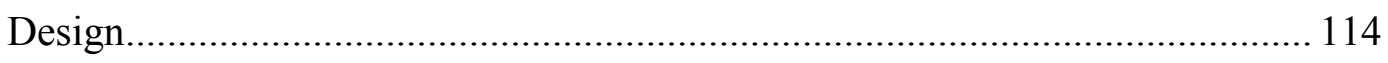

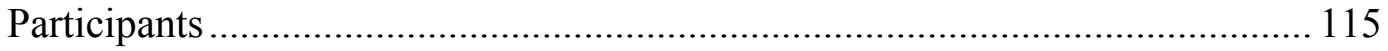

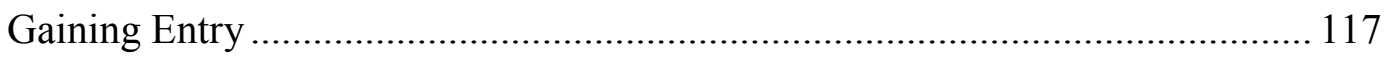

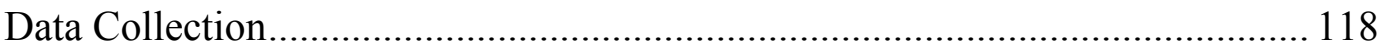

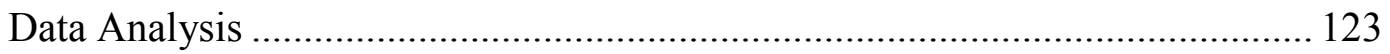




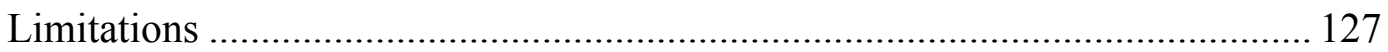

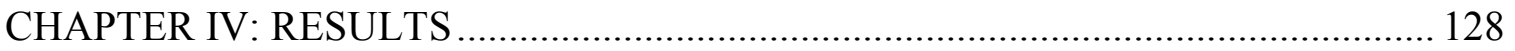

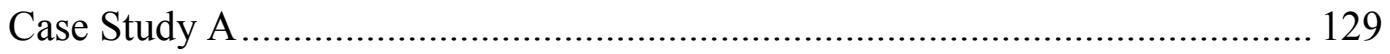

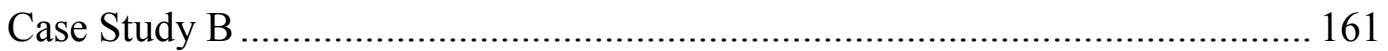

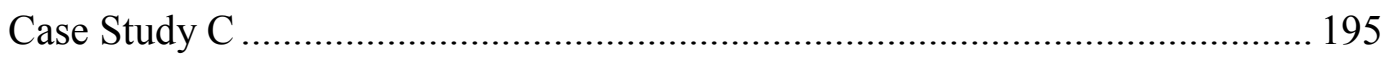

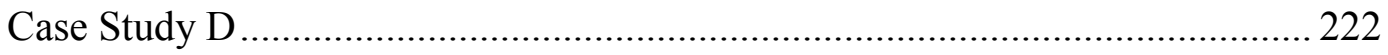

CHAPTER V: DISCUSSION, IMPLICATIONS, AND CONCLUSIONS .................. 250

Summary of Findings and Cross-Case Analysis ........................................ 250

Linkages to Previous Literature ............................................................... 273

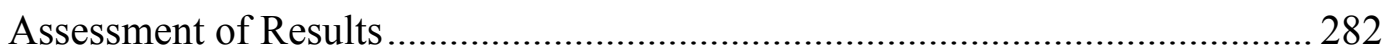

Suggestions for Education Stakeholders and Researchers ............................... 288

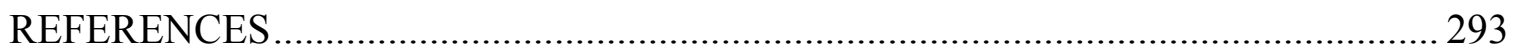

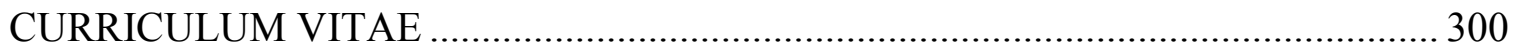




\section{LIST OF FIGURES}

FIGURE

PAGE

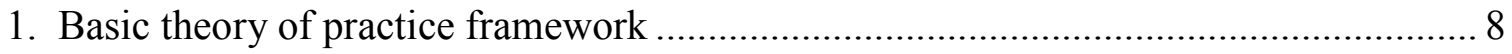

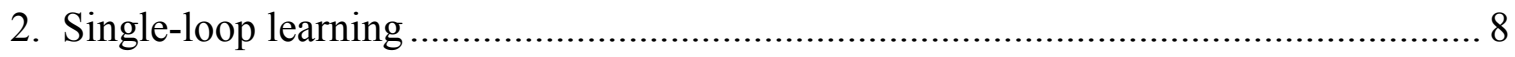

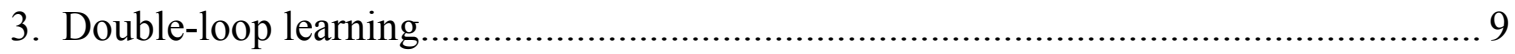

4. How policy mandates fail to raise student achievement ............................................ 108

5. Conceptual framework: How principal reflective practice might raise

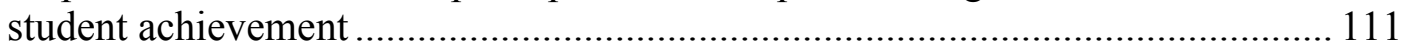

6. Visual representation of each theory of practice framework ……............................ 125

7. Case study A: Theory of practice for meeting individual student needs ................... 133

8. Case study A: Theory of practice for nurturing relationships ................................... 142

9. Case study A: Theory of practice for encouraging continuous professional

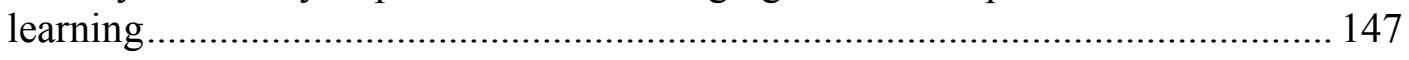

10. Case study A: Theory of practice for inviting teacher input.................................. 152

11. Case study A: Theory of practice for using directive leadership ............................ 156

12. Case study B: Theory of practice for nurturing a positive learning climate ........... 164

13. Case study B: Theory of practice for inviting teacher input .................................. 172

14. Case study B: Theory of practice for giving teachers autonomy ............................. 177

15. Case study B: Theory of practice for encouraging teacher collaboration................ 179

16. Case study B: Theory of practice for maintaining a school-wide focus on curriculum and instructional improvement ................................................... 185

17. Case study B: Theory of practice for providing feedback ……………………....... 191

18. Case study C: Theory of practice for nurturing positive relationships ………........ 198

19. Case study C: Theory of practice for unifying staff.............................................. 204

20. Case study C: Theory of practice for providing feedback ……………………......2210 
21. Case study C: Theory of practice for encouraging continuous

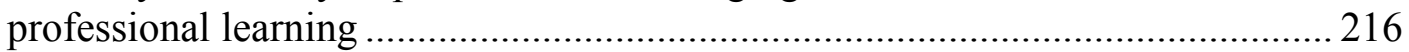

22. Case study C: Theory of practice for inviting teacher input and encouraging teacher autonomy

23. Case study D: Theory of practice for encouraging continuous professional learning...

24. Case study D: Theory of practice for inviting teacher input................................. 239

25. Case study D: Theory of practice for engaging individual students 


\section{CHAPTER 1}

\section{INTRODUCTION}

School reform efforts are fraught with difficulty. As Bassett (1998) noted, "Change comes to the universe of schools slowly, if at all, and only after perturbations that rock the firmament" (p. 1). But even when those "perturbations" come in the form of enormous accountability pressures imposed by state and federal government mandates, schools continue to operate largely as they always have.

This modern era of government policy-driven education reform began with the 1983 publication of $A$ Nation At Risk, the report of the National Commission on Excellence in Education (NCEE), and was followed by a plethora of state-level accountability and reform efforts such as the Kentucky Educational Reform Act (KERA) in 1990. Perhaps the pinnacle of these mega-policy reforms was the No Child Left Behind (NCLB) Act of 2001, which tied federal education funding to specific outcomes in student learning.

Despite these sweeping policies, however, schools remained highly resistant to change and student achievement remains stagnant (National Center for Educational Statistics, 2006). The explanations for change-resistance and low achievement are many, but several authors indicate that there is an abiding schism between theory and practice on the part of teachers and principals (Keedy, 2005; Keedy \& Achilles, 1997). Educators themselves remain cut off from the reflection, research, collaboration and experimentation describing schools that have become the centers of change, rather than 
targets of change (Sirotnik, 1989). Moreover, Cusick (1992) found the overwhelming orientation of school personnel was an obsession with control of the various unpredictable inputs in the educational process, a viewpoint that does not lend itself to the risk-taking and experimentation associated with change-oriented environments.

\section{Background of the Study}

A Nation at Risk (1983) blasted public schooling in America as a dismal failure and became a landmark document, fueling a wide variety of school reform efforts called "the greatest and most sustained concerted national effort to change the central core of assumptions and structures of the public schools in the history of the Republic" (Owens, 2004, p. 45). A multiplicity of new laws and mandates followed at both the state and federal levels to improve teacher training, to restructure the organization of schools, to standardize curricula and, above all, to hold teachers, principals and school district leaders accountable to improving student achievement in measurable ways, culminating in the No Child Left Behind Act of 2001.

Despite these massive reform efforts, many educators found little lasting change in American schools. Gordon (2003) argued that the policy-mandated focus on accountability testing made a limited impact on what happened in most classrooms. The National Assessment of Educational Progress (NAEP), administered to students nationwide at ages 9, 13, and 17, supported Gordon's assertion. NAEP data indicated little if any growth in average student scale scores in reading and math from the early 1970s until the present day (National Center for Educational Statistics, 2006).

Gordon (2003) identified teacher isolation from other educators as one of the explanations for this lack of progress. Gordon's conclusions were consistent with those 
of Keedy and Achilles (1997), who argued that structural changes had a limited impact on improving student achievement and that a shift in normative thinking (the school as a "collectivity's" values, beliefs and assumptions that are the actual guides to daily decision making in schools) must first be made to create school climates that foster critical self-reflection and the testing of new assumptions to bring about improvements in student performance. "There is precious little evidence that students will become thoughtful, independent learners through legislated external pressure," Keedy and Achilles concluded (p. 5).

The mega-policy emphasis on one-size-fits-all educational mandates misses the real issue of why schools do not change. Writing just a few years after $A$ Nation at Risk was published, Sirotnik (1989) argued that schools were difficult to change because they are not themselves places of inquiry and self-discovery. Epistemological and organizational issues in the everyday work lives of teachers and principals prevent educators from really engaging in deep self-reflection and theory development. Until schools become centers of inquiry, they cannot become centers of change. The decentralized nature of public schools dictates that schools themselves must become the centers of change, rather than the targets of change from outside forces, if lasting reform is going to occur. Cusick (1992) indicated that a climate of inquiry is highly unlikely in most schools, given that the nature and logic of school organizations focuses primarily on the issue of control.

Using descriptive studies from two decades of qualitative research, Cusick (1992) described the various subgroups of individuals — students, teachers, administrators, outside parties, and government and reform groups — and how the inter-dynamics of these 
various groups created a system with its own internal logic and rules of order. He found that the overarching theme of the entire educational system was the need for control. This need emerges from the many uncontrollable variables that are involved in the educational process - innate student abilities, socio-economic barriers to learning, limited resources, the many competing political and social values that students bring to school with them and that lead to a plethora of pressures from groups outside the formal system, and above all the tendency of students to form their own groups and operate according to value systems that may be entirely at odds with the values the school wants them to learn and operate by.

Because the teacher must be concerned primarily with control of events in his or her classroom, teachers begin to work in isolation from one another. "A professional distance [between teachers] is maintained," Cusick wrote. "Each [teacher] has the problem of imposing the school's definition of reality onto students. This is a personal problem and teachers solve it personally" (p. 96).

This isolation not only shapes the relationships of teachers with one another, but of teachers and their administrators. Principals need teachers to control the events within their classrooms and teachers need principals to control as many variables outside the classroom as possible to keep them from spilling through the classroom door. Thus, the teachers' primary expectation for principals is the smooth, orderly operation of the school's day-to-day affairs, rigorous discipline of unruly students, and protection from aggressive parents. The result for the principal, like the teacher, is a carefully nuanced emphasis on control. "Operationalized, school administration is the taking of one of the innumerable elements that is or threatens to be out of kilter with the others and putting it 
back into the routine, or even fitting something new into the routine" (Cusick, 1992, p. 124).

Keedy and Achilles (1997) and Keedy (2005) suggested that development of theories of practice by school administrators could become a central vehicle for facilitating change in schools. Such an approach is congruent with the idea of schools as centers of inquiry (Sirotnik, 1989) and would have definite implications for the control orientation of schools (Cusick, 1992). Keedy and Achilles (1997) argued that the collective values, beliefs and assumptions of teachers and administrators significantly mediate the long-term effectiveness of structural changes in schools, such as site-based management and reforms in teacher professional development and instructional practice. The authors argued that changing relationships among education stakeholders is the key litmus test for the effectiveness of school restructuring efforts:

Classrooms in the USA are, generally, boring places; the modal number of questions that students ask of their teachers per high school class period is two. Sizer puts it bluntly: teachers, rather than students "do the work"... [Students] are not engaged in the material and do not have to make their own meanings. (Keedy \& Achilles, 1997, citing Sarason, p. 2)

Keedy and Achilles (1997) contrasted this kind of student-teacher relationship with the kinds of relationships one would expect to find in a restructuring school, characterized by “compassion, cooperative effort, student mindfulness and mutual respect for articulate and diverse positions on crucial issues" (p. 3). Likewise, the authors argued that relationships among teachers and between teachers and principals are also transformed in a genuinely restructuring school, taking on more collaborative approaches to decisionmaking and marked by trust, openness and mutual support for self-inquiry and selfdevelopment. This kind of relationship stands in stark contrast to the kinds of teacher- 
teacher and teacher-principal relationships described by Cusick (1992), which are focused on isolation and could be described as "you do your job and I'll do mine."

Keedy (2005) outlined how this historic lack of reflection among educators emerged from the Positivist and Technical-Rationality movements in the late $19^{\text {th }}$ and early $20^{\text {th }}$ century and documents how these philosophies contributed to an everdeepening schism between theory and practice, both in the training of education administrators and in the schools where they serve. That schools are not places of critical self-reflection is therefore not a new problem, nor are some of the possible solutions to the theory-practice schism new. However, the emphasis on structural changes to improve schools has distracted teachers and principals from the real work of questioning normative thinking that is necessary for healing the theory-practice divide.

The key vehicle that Keedy and Achilles (1997) proposed for changing the normative thinking of schools and thereby bridging the gap between theory/research and practice is the development of self-reflective, teacher and principal-oriented theories of practice, in which "practitioners actively and persistently analyse their assumptions in relation to the grounds supporting their practice" by critiquing the assumptions that lie beneath their decisions and actions, articulating alternate assumptions, values or beliefs, and testing the effectiveness of these new assumptions in the context of their own work environments, i.e., their schools and classrooms" (p. 5). The results of testing these new "theories of action" inform a continuous loop of inquiry, testing, learning, and inquiry. This approach was first systematically operationalized by Argyris and Schön (1974) and utilizes a system of "double-loop learning" that has the potential to expose and transform normative thinking in the way Keedy and Achilles suggested. 
In their text Theory in Practice (1974), Argyris and Schön identified models for how effective and ineffective learning takes place within individuals and groups. According to Argyris and Schön, individuals possess "theories in action," which are the mental maps we use to negotiate a wide variety of problems we encounter in our daily lives.

Theories of action may fall into two categories: theories in use and espoused theories. Theories in use are containers for a multitude of attitudes, beliefs and values that guide and motivate our decisions. They "contain assumptions about self, others and the environment-these assumptions constitute a microcosm of science in everyday life" (Argyris \& Schön, 1974, p. 30). Because they contain a deeply psychological component, theories in use often manifest as defense mechanisms designed to protect our self-esteem or hide our true feelings from self or others. These theories in use may or may not be conscious to the individual and may even be at odds with their "espoused theories," which are the ways in which we explain our decision-making processes to others (see Figure 1).

Because theories in use are (a) so deeply entrenched in the individual psyche, (b) often subconscious to the individual, and (c) often at odds with espoused theories of action (how we say we behave to others or how we rationalize our behavior to others), they deeply affect the way individuals learn. Argyris and Schön (1978) described the typical, reflexive way we learn as single-loop learning, in which the individual sees that his or her behavior has not successfully resolved a problem. In single-loop learning, the individual then adjusts the action strategy to achieve a different outcome without ever questioning the underlying values and assumptions about the situation (see Figure 2). 


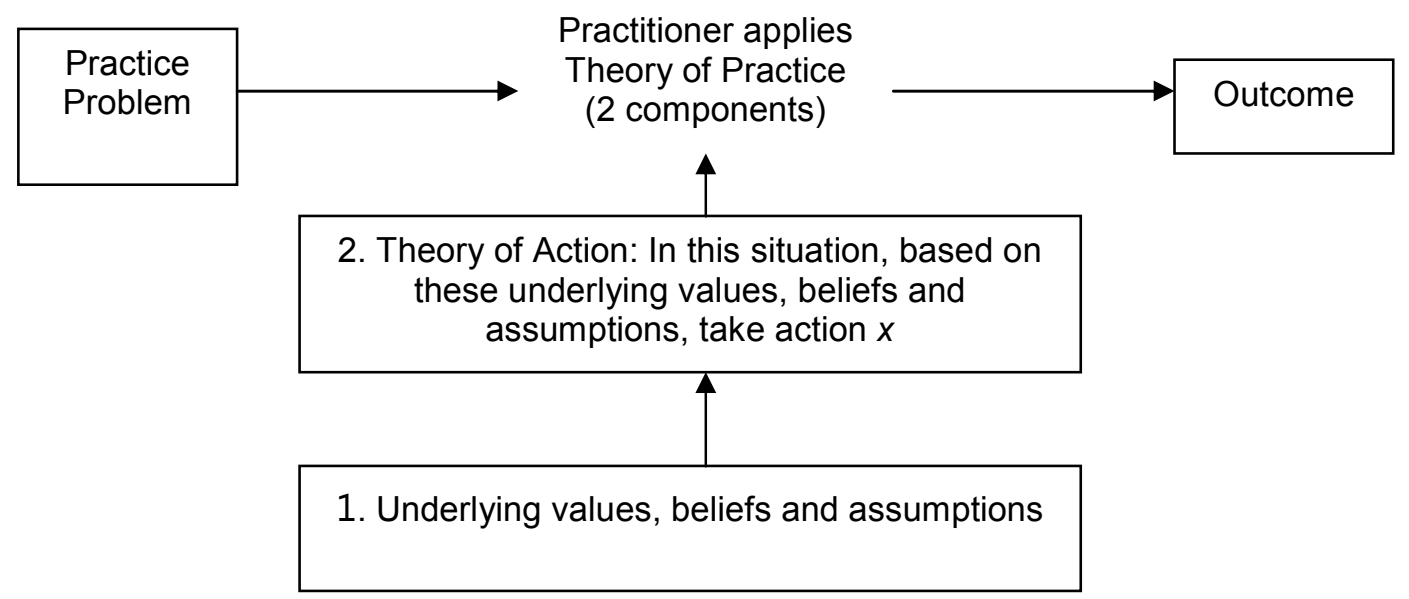

Figure 1. The basic theory of practice framework demonstrates how the practitioner responds to a problem with a theory of action based on a set of underlying values, beliefs and assumptions, to achieve a desired outcome.

In double-loop learning, on the other hand, the failure of a particular action to achieve the desired result will lead not only to a re-evaluation of the action strategy itself, but also the values, principles and assumptions the person possesses that affect the way action strategies are developed in the first place. They found double-loop learning to be superior in that it allows far more creativity and flexibility in developing new strategies to

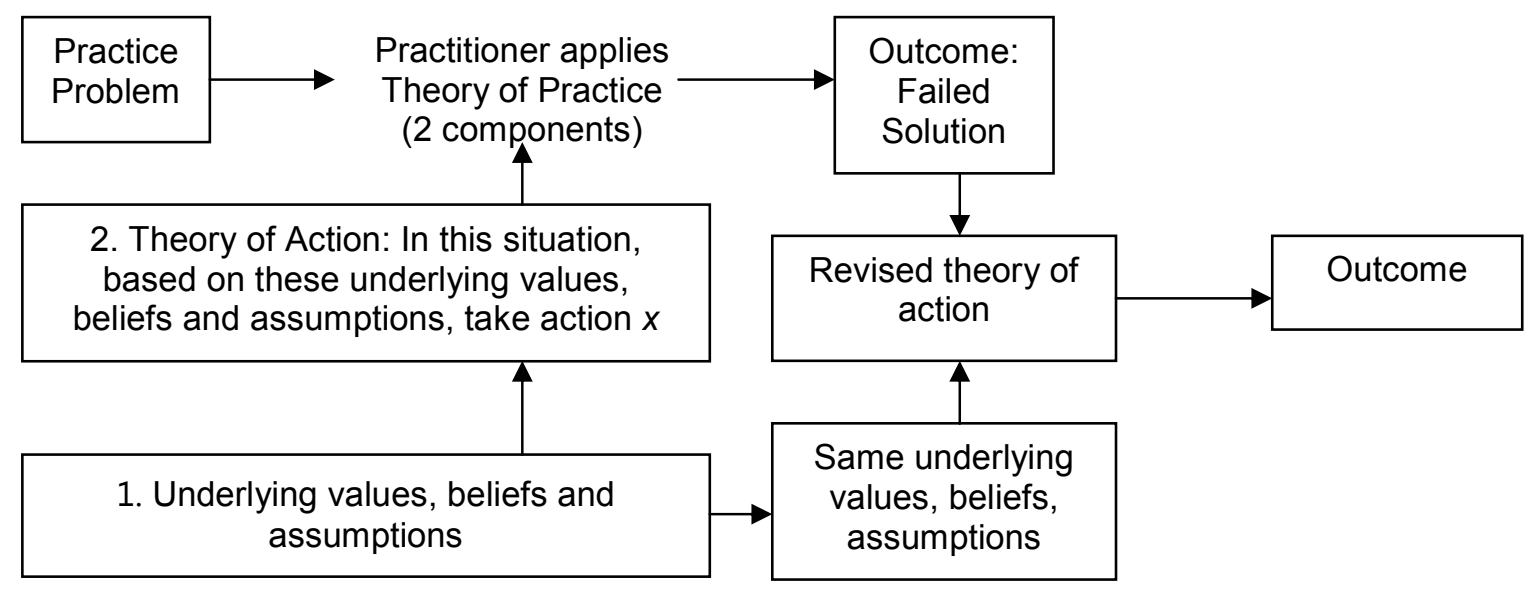

Figure 2. Single-loop learning, reflecting a revised theory of action based on the original set of underlying values, beliefs and assumptions. 
address the ever-changing problems presented by constantly-shifting contexts and circumstances (see Figure 3).

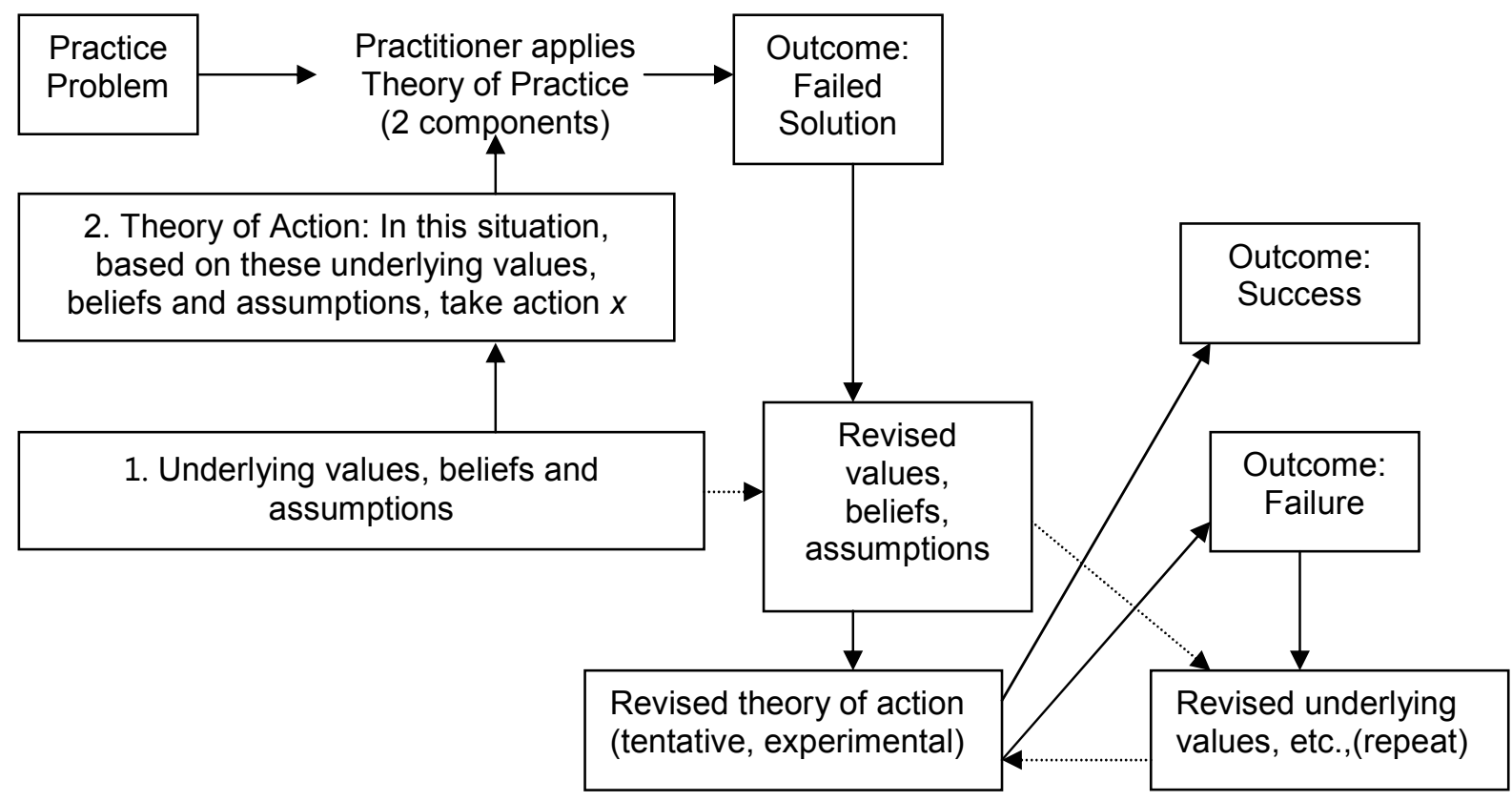

Figure 3. Double-loop learning, where a new theory of action is developed based a revised set of values, beliefs and assumptions (the Reflective Practitioner).

Theories in use may then either inhibit double-loop learning (which the authors described as Model I behavior) or enhance it (Model II). Model I behavior, according to Argyris and Schön (1974), contains the implicit assumption that all problems involve a win-lose outcome for individuals. Thus, Model I behavior involves an attempt to control the external circumstances of a situation and avoid the vulnerability of making one's feelings and internal motivations known to others. Defensive behavior that reinforces the individual's underlying assumptions is the primary component of Model I. Argyris, Putnam, and Smith (1985) argued that Model I behavior discourages inquiry and promotes defensive relationships, results in low freedom of choice, reduces production of valid information and provides little public testing of ideas. 
Model II behavior, by contrast, encourages double-loop learning, inquiry and questioning of values and assumptions, and the deliberate identification and testing of theories in use. In an organizational context, Model II typically involves shared leadership, open, dialogical processes of problem-solving and participatory decisionmaking. In practice, this means carefully bringing stakeholders into open discussion of problems, making oneself vulnerable by identifying one's underlying motivations and values, even when the outcome is unknown, collectively articulating strategies of action for addressing the problem, gathering data to test the effectiveness of the outcome, and reflecting on the outcome's implications for our underlying assumptions and action strategies in an on-going process.

The present study explored how successful principals used theories of practice to shape their instructional leadership. Argyris and Schön (1974) first articulated the concept of theories of practice and Schön (1983) later developed this construct into the broader concept of reflective professional practice. The present study investigated the ways in which principal theories of practice influence the attitudes and perceptions of teachers in successful schools, and explored the extent to which the case study principals engaged in double-loop learning and reflective practice. If successful principals can effectively use theories of practice to encourage teacher self-reflection and professional growth and to mitigate the negative, isolating effects of the control orientation, then both practicing principals and students in schools of educational administration could benefit from deeper levels of reflective practice. By reflectively using theories of practice, principals might foster school climates more aligned to Sirotnik's (1987) idea of schools as "centers of inquiry" with a far greater capacity for increasing student achievement. 


\section{Research Problem}

In response to accountability pressures, Kentucky, like many other states, established goals for each school to improve student achievement at yearly and biannual increments. Poor student performance can lead to a variety of sanctions and school principals are personally responsible for student achievement (Pankratz \& Petrosko, 2000). The federal government ties educational funding to schools demonstrating adequate yearly progress in student achievement (NCLB, 2001).

Despite these pressures, however, schools remain highly resistant to the relational changes necessary for promoting student achievement. Without changes in norms of behavior, including more open, trusting relationships among school staff, schools are likely to remain characterized by professional isolation and a strong control orientation (Cusick, 1992; Keedy \& Achilles, 1997). Research indicates that school principals play a key, if indirect role, in promoting higher student achievement (Hallinger \& Heck, 1998; Marzano, Waters, \& McNulty, 2005;Witziers, Bosker, \& Kruger, 2003).

Principals have a positive impact on school culture by encouraging teacher empowerment through self-managed teacher leadership teams (Davis \& Wilson, 2000; Short, 1994), encouraging teacher networking and building relationships of trust (Spillane \& Thompson, 1997), engaging in specific instructional leadership practices that increase teacher motivation and self-efficacy (Basom \& Frase, 2004; Blase \& Blase, 1999), and sharing leadership and being open to reciprocal influences from effective teacher leaders (Anderson, 2004; Keedy \& Simpson, 2001). Schools are essentially sets of interconnected relationships (Keedy \& Achilles, 1997). By changing the nature of 
relationships, the school itself is changed, and the trust and openness necessary to becoming a center of inquiry is created.

\section{Rationale}

Principal use of theories of practice, especially regarding the principal's role as instructional leader of the school, could be a mechanism for promoting these new norms of behavior and relationships of openness and trust. Empirical studies applying Argyris and Schön's (1974) models of reflective practice accurately describe the professional behavior of teachers (Ferry \& Ross-Gordon, 1998; Kirby \& Paradise, 1992; Kirby \& Teddlie, 1989; Tsangaridou \& O’Sullivan, 2000), and have promise for improving the effectiveness of principals (Erlandson, 1994; Polite, 2000). However, the scope of these studies was limited and further research on school principals' use of reflective practice is needed.

\section{Purpose of the Study}

This study investigated how reflective principals in successful Kentucky schools used theories of practice to shape their instructional leadership, the impact of those theories of practice on teachers, and the extent to which the principals engaged in doubleloop learning and reflective practice. If principals can utilize theories of practice to enhance their instructional leadership and promote a climate for higher student achievement, this study may point the way to further research and training for principaldeveloped theories of practice.

Area superintendents and district administrators nominated the selected principals for being self-reflective about their instructional leadership. The principals presided over increases in student achievement as measured by Kentucky's state-wide accountability 
test, the Commonwealth Accountability Testing System (CATS) over a two biennia period. Specifically, these schools met or exceeded their CATS goals as established by the state for two consecutive biennia.

There are many reasons for school success. This study focused on the relationships between teachers and principals in successful schools and how the principals' theories of practice regarding instructional leadership shaped those relationships. Three central research questions framed the study:

1. What are the theories of practice of instructional leadership for these principals?

2. How do principal theories of practice regarding instructional leadership affect teachers?

3. Does their use of theories of practice conform with Argyris and Schön's conception of double-loop learning and Schön's conception of the "Reflective Practitioner?"

\section{Definition of Terms}

\section{Espoused Theories and Theories in Use}

Terms related to theories of practice include espoused theories and theories in use. According to Argyris and Schön (1974), an espoused theory is simply the explanation a professional gives for how he or she typically solves specific problems in the workplace, "the theory of action to which he [sic] gives allegiance, and which, upon request, he communicates to others" (p. 7). Theories-in-use, on the other hand, are the theories that actually govern a person's behavior and can be constructed only by observation of the person's behavior. A common issue in studying theories of practice, according to Argyris and Schön, is the inconsistency between a professional's espoused 
theories and theories-in-use. This study seeks to clarify the congruence of espoused theories and theories-in-use of the participants.

Instructional Leadership

DeBevoise (1982) offered an early definition of instructional leadership as "those actions that a principal takes, or delegates to others, to promote growth in student learning" (p. 14). A wide variety of behaviors fall within this definition, and Cuban (1984) acknowledged the difficulty in identifying specific instructional leadership behaviors as opposed to non-instructional behaviors on the part of principals. Wildy and Dimmock (1993) clarified the definition of instructional leadership to six specific sets of principal activities: (a) defining the purpose of schooling; (b) setting school-wide goals, (c) providing the resources needed for learning to occur, (d) supervising and evaluating teachers, (e) coordinating staff development, and (f) creating collegial relationships with and among teachers. Blase and Blase (1998) identified a shift in thinking about instructional leadership over the last few decades from one of instructional supervision, which implied more autocratic, top-down approaches to decision-making, to more open and collaborative approaches which promoted self-reflection and a desire for professional growth on the part of teachers. Based on these descriptions of instructional leadership, this study defined instructional leadership as principal behaviors which were meant to promote higher levels of student achievement through the principal's interactions with teachers.

\section{Theories of Action}

According to Argyris and Schön (1974), theories are "vehicles for explanation, prediction, or control" (p. 5). All humans, whether they are conscious of it or not, 
operate according to thousands of theories to explain their experience, predict future events, and control outcomes in various situations. All theories are situational, and based on an underlying set of values, beliefs and assumptions that frame an individual's perception of the world, which include assumptions about desirable outcomes for a variety of situations. Thus, theories appear in an "if . . . then" format: if the individual faces a particular situation, then based on the individual's values, beliefs and assumptions about this situation, the individual should then take a particular action to either explain, predict or control the situation or outcome. Argyris and Schön called this if-then formulation a theory of action. "A full schema of a theory of action, then, would be as follows: in situation $S$, if you want to achieve consequence $C$, under assumptions $a_{1} \ldots$ $a_{n}$, do $A$ " (p. 6).

Theories of Practice

Argyris and Schön (1974) went on to define theories of practice as "special cases" of theories of action that are rooted problems arising in a professional's specific work context. Theories of practice describe routines, procedures and specific practices for dealing with problems common to the practice environment. "A practice is a sequence of actions undertaken by a person to serve others, who are considered clients. Each action in the sequence of actions repeats some aspect of other actions in the sequence, but each action is in some way unique. In medicine, for example, a typical sequence would be a diagnostic work-up, treatment of acute illness, a well-baby visit, chronic care, and consultation" (p. 6).

A theory of practice, then, consists of a set of interrelated theories of action that specify for the situations of practice the actions that will, under the relevant assumptions, yield intended consequences. Theories of practice usually contain theories of intervention - that is, theories of action aimed at enhancing 
effectiveness; these may be differentiated according to the roles in which intervention is attempted - for example, consulting and teaching. (p. 6)

This study explored the theories of practice of successful principals with regard to their role as instructional leader-their assumptions and theories of action for confronting and solving problems of instruction, learning and student achievement in a school context unique to the role of school principal.

\section{Reflective Practitioner}

Schön $(1983,1987)$ refined the concept of theories of practice by describing how a professional utilizes theories of practice to intentionally and reflectively question one's assumptions and theories of action in a process called "reflection-in-action." According to Schön, six indicators describe the reflection-in-action process: a) recognition of the problem; (b) recognition of incongruities between one's espoused theories and theoriesin-use; (c) evidence of reframing of the problem; (d) generation of new solutions; (e) testing-in-action of solutions; and (f) evaluation of outcomes. Principals who utilize these processes are considered reflective practitioners.

A review of literature that frames this study in Chapter II begins with an overview of mega-policy school reform efforts, followed by a discussion of literature on the change resistance of schools, the role of school principals in promoting higher student achievement, and research on educator theories of practice. Chapter II concludes with a conceptual framework for this study. 


\section{CHAPTER II \\ LITERATURE REVIEW}

This review of literature includes five major sections. The first section describes major school reform efforts, including the report A Nation at Risk (1983), the Kentucky Education Reform Act (1990), the federal No Child Left Behind Act (2001), and the role of School-Based Decision-Making (SBDM) councils in Kentucky's school reform efforts. The second section explores research on the relationship between principal behavior and student outcomes. The next section explores literature on the resistance to change within schools and how the nature and logic of schools must be transformed through effective leadership in order to affect improvements in student learning. Section four examines specific research studies on the principal's role in transforming relationships within schools. The last section describes empirical literature on the concept of theories of practice, first articulated by organizational behaviorists Argyris and Schön (1974), which might point the way toward enhancing instructional leadership for school improvement. The chapter concludes by articulating a conceptual framework of how theories of practice of instructional leadership may serve as a bridge to help principals foster more open, collaborative relationships in their schools and thereby enhance student achievement. 


\section{Education Reform: Standardization \& Decentralization}

For at least two-and-a-half decades, education in the United States has largely been shaped by federal and state mega-policy reform efforts. Policy-makers designed these initiatives to improve student achievement by standardizing curricula, holding schools accountable through high-stakes achievement testing, and restructuring education through a variety of changes in funding and governance of public schools. The last point - school governance - suggested that despite the one-size-fits-all approach to curriculum and assessment of these policies, education reformers believed that decentralization of decision-making power would be a key component in helping schools meet these accountability standards. This section is divided into two subsections: (a) an exploration of federal educational reform initiatives, embodied in the 1983 report $A$ Nation at Risk and the 2001 reauthorization of the Elementary and Secondary Education Act, better known as the No Child Left Behind (NCLB) Act; (b) a focus on the Kentucky Education Reform Act (KERA) of 1990, an early example of a state-level reform effort that embodied both standardization of curriculum and assessment and decentralization of decision-making authority within the schools.

\section{Federal Initiatives: A Nation at Risk and NCLB}

In 1983, Secretary of Education Terrell Bell assembled the National Commission for Excellence in Education (NCEE) and released a report entitled A Nation at Risk: An Open Letter to the American People. The report sounded an alarming call for drastically improving the state of education in America, and argued that the contemporary situation paralleled 1957, when the Soviet Union launched the satellite Sputnik and called into question the United State's technological and scientific superiority. The current 
education situation, the report argued, threatened to shove the U.S. into a state of economic and political collapse (NCEE, 1983).

A Nation at Risk (NCEE, 1983) citing student test data compared with other nations, the increase in college remedial courses, and a host of data from other reports and assessments, called for substantial educational reform and recommended four main areas of concern having to do with curriculum, expectations for student achievement, the use of instructional time, and assessment. The report recommended that schools increase their graduation requirements and that elementary and secondary curricula be standardized and made more rigorous. The authors called for higher academic expectations for student performance, grading practices that reflect real student achievement, standardized testing at key transition points to monitor student progress, and more instructional time through longer school days and years. Finally, A Nation at Risk suggested improvements in teacher preparation programs, higher requirements for admission into teacher certification programs, mentoring and internship plans for new teachers, competitive teacher pay based on student outcomes, and involvement of citizens in oversight of reform efforts and in school governance and financing decisions.

The NCEE report was controversial when it appeared in 1983 and remains so (Bracey, 2003; Gardner, 1984; Guthrie \& Springer, 2004; Holton, 2003; Howard, 2003; Voskuil, 1999), but there is no question that $A$ Nation at Risk ushered in a new era of major school reform efforts at both the state and federal level. Many professional educators and researchers met the report with great skepticism and criticism. Writing soon after the report's release, Gardner (1984) argued that $A$ Nation at Risk reflected a "lack of critical analysis" of the real issues involved in public education (p. 13). Others 
argued that educational performance was often tied with a nation's economic growth, and the U.S. in 1983 faced a serious recession (Voskuil, 1999). Still others questioned data used in the report and the limited perspective of the commission members. Bracey (2003) noted that some of the commissioners had visited only one other country (Japan) in their assessment of U.S. achievement relative to other nations. Finally, others perceived a widespread perception that political agendas to weaken public education motivated the report (Guthrie \& Springer, 2004).

State and federal governments responded to the report with a plethora of new reform initiatives motivated by the goal of improving student achievement by moving "away from measuring the quality of the schools by the resources they receive" to "a plane where school performance is judged on outcomes students' achieve" (Guthrie \& Springer, 2004, p. 9). Given the decentralized nature of education in the U.S., and that historically states have taken most of the responsibility for public schooling, it is not surprising that states would take the lead. However, frustrated with the slow and uneven progress of educational reform in the states, in 2001 federal lawmakers took matters into their own hands during the reauthorization of the Elementary and Secondary Education Act, which allocates federal money for education to the states. Dubbed the No Child Left Behind (NCLB) Act, the sweeping new legislation tied federal education dollars to improved student achievement.

NCLB required states to do the following to continue receiving federal assistance: (a) ensure that only "highly-qualified" teachers teach classes, (b) use only research-based practices for improving student achievement, and (c) use standardized testing procedures for reading and math in grades 3,6 , and 8 to demonstrate adequate yearly progress (AYP) 
in improving student achievement and closing gaps in achievement of various student sub-groups based on ethnicity, socioeconomic, language and disability status (NCLB, 2001). Schools that fail to make AYP face sanctions and are eligible for supports to make improvements or lose federal education funding. Far from Ronald Reagan's ideas of scaling back federal involvement in education, NCLB became the largest mega-policy approach to school reform in the nation's history, and had a major impact on state-level reform efforts, including in states like Kentucky that already had long-standing school improvement efforts underway.

The Kentucky Education Reform Act (KERA) and School-Based Decision Making In 1989, a group called the Coalition for Better Schools representing 66 of the 176 school districts in Kentucky sued the state government, arguing that Kentucky's propertytax-based system of school financing was illegal given that the state constitution required an "efficient system" of public education for all students in the Commonwealth. The Kentucky Supreme Court agreed with the plaintiffs, ruling the state's school funding system unconstitutional and ordered the state legislature to revise the system accordingly (Rose v. Council for Better Education, Inc., 1989). The next year the General Assembly passed the Kentucky Education Reform Act (KERA) of 1990, "one of the most farreaching state educational reform efforts in recent history" (Petrosko, 1993, p. 4).

Among its many provisions, KERA (a) established a funding formula guaranteeing a minimum amount of state education dollars per student, adjusted for a district's particular needs, (b) set academic expectations for student learning in six different areas, (c) standardized a state curriculum for all students, (d) established a statewide, criterion-referenced testing system, the Kentucky Instructional Results Information 
System (KIRIS), later revised to the Commonwealth Accountability Testing System (CATS), with the goal that all students would perform at proficient levels in all content areas by the year 2014, (e) set goals for increased attendance and successful transitions to adult life and reduced grade retentions and dropouts, (f) increased professional development for teachers, (g) established funding for preschool education and Family Resource and Youth Service Centers in low-income districts, and (h) established a system of rewards and penalties for schools that succeeded or failed in meeting their goals (Kentucky Department of Education, n.d.). With the advent of the No Child Left Behind Act, the state legislature revised KERA to align with goals of NCLB, expanded its testing system and established goals for making adequate yearly progress (AYP) based on NCLB measures (KDE, 2005).

A key component of KERA included the establishment of School-Based Decision Making (SBDM) Councils. The law required that most Kentucky schools set up a council comprised of the principal and a specific formula of parents and teachers, with appropriate minority representation when the diversity of a school's student body met a certain threshold. The SBDM Councils' responsibilities included a variety of governance decisions, such as budgets, staff assignments (including hiring principals), professional development, curriculum and establishing, monitoring and revising a Comprehensive School Improvement Plan (CSIP).

Björk and Keedy (2003) saw the move toward decentralization of decisionmaking in Kentucky as "a leitmotif of a larger score being played out in education reform in the United States" (p. 34). They identified a widespread consensus of both progressive and conservative education reformers that decentralization of authority within the schools 
constituted a necessary element to empowering teachers and overcoming the culture of isolation, control and hierarchy that characterized schools in the U.S. (Cusick, 1992; Keedy \& Achilles, 1997). Empirical studies, however, have found that school-based decision making effects are "far from compelling," though Kentucky's form of decentralization "appears to hold the greatest promise for student growth" (Leithwood \& Menzies, 1998, p. 235).

Leithwood and Menzies (1998) conducted a review of 77 empirical studies on SBDM effects between the years 1985 and 1995 to identify obstacles SBDM councils faced in successfully implementing decentralization of decision making. The researchers found that the most widespread obstacle faced by councils was "interpersonal conflict," and that when teachers and principals adhered to their traditional decision-making roles, the effectiveness of councils was extremely limited. They concluded from this review of literature that SBDM success depended on deliberate efforts to promote non-traditional models of leadership and principal efforts to share power. Principals have "an especially crucial role" in the implementation and outcomes of school-based decision making, (Leithwood \& Menzies, 1998, p. 236).

Klecker, Austin and Burns (1999) found that SBDM Councils in Kentucky often focused on non-instructional concerns, which may limit their effectiveness in promoting student achievement. Klecker et al. (1999) examined the status of implementation of SBDM councils in Kentucky and the types of decisions made by SBDM councils during the 1996-1997 school year and part of the 1997-1998 school year. The population for the study included school councils from 1,032 schools in Kentucky identified as having SBDM Councils in October 1997. The researchers generated a stratified random sample 
of 344 schools representing elementary, middle and high schools from throughout the state, and an achieved sample of 137 councils (40\%) responded to the researchers' request to participate in the study. The chi-square goodness of fit statistic confirmed that the achieved sample still adequately represented the population.

Klecker at al. (1999) reviewed demographic information for each council and agendas and minutes from council meetings from July 1, 1996 to November 30, 1997. The researchers coded data for analysis using thirteen categories, including the nine categories of responsibility for SBDM Councils mandated by state law. A second researcher categorized a random sample of data to strengthen the trustworthiness of researcher interpretations, with an inter-rater reliability of .93. With categorical variables such as region, school level, and length of principal tenure serving as independent variables, the researchers conducted t-tests and analysis of variance (ANOVA) to determine if significant differences existed among groups in terms of the categories of decisions that dominated the council agendas.

Among the findings, Klecker et al. (1999) discovered no significant relationship between principal-level factors and SBDM Council decisions. Elementary councils made fewer decisions about curriculum than high schools, but high school councils made more decisions about discipline and personnel than elementary. Regardless of school level, councils made significantly more decisions about budgeting, procedures and personnel than about curriculum and instruction.

Din (1997) laid the groundwork for Klecker et al. (1999) by examining categories of decisions made by SBDM Councils in Kentucky's rural districts. Din surveyed a stratified random sample of SBDM Councils in rural Kentucky districts. A response rate 
of $52 \%$ gleaned an achieved sample of 132 schools. The researcher surveyed school council members using an instrument to measure the extent to which councils worked toward the sixteen "missions" for SBDM Councils identified in state law. Results indicated that rural councils in Kentucky spent, on average, $34 \%$ of their time on instructional issues, with a range of $5 \%$ to $90 \%$, and respondents indicated that lack of instructional focus was a key challenge their councils faced. Din concluded that in many cases principals held on to decision-making power within schools despite the presence of SBDM Councils, and that the inconsistency in an instructional focus across rural schools was a key challenge to Council effectiveness in improving student achievement. In surveying research on the limited effects of school councils on student achievement, Björk and Keedy (2003) found that councils struggled to recruit parents and teachers, especially those with the most experience, perhaps in part because of the time commitment involved and lack of instructional focus (Björk \& Keedy, citing Newton, Keedy, \& Winter, 2001; Logan, 2000; Winter, Keedy, \& Newton, 2000). In summary, Kentucky's educational reform efforts mirror the mega-policy initiatives called for in A Nation at Risk and embodied in the federal No Child Left Behind Act and suggest that decentralized decision making at the local level is a key to successfully meeting the goals of these accountability efforts. There is a question, however, about how much either the centralization of accountability or the decentralization of decision making have impacted student achievement. Literature reveals that a key variable in school effectiveness, including the success of SBDM Councils, is the principal. The next two sections of this literature review explore 
empirical research on the effects of principals on student achievement and in shaping school culture.

\section{Principal Effects and Student Achievement}

The previous section outlined some of the most significant school accountability efforts at both the national and state level. These efforts put great pressure on schools to improve student achievement. The school principal has a central responsibility in governing and leading schools in such a way that students can learn at ever higher levels. This section reviews the literature on principal effects and student outcomes. Early studies struggled to articulate a definition of "instructional leadership" so that researchers knew what exactly to measure relative to student achievement, and found primarily indirect effects and accounted for only a small amount of the variance in student outcomes. Recent studies such as the one conducted by Marzano, Waters, and McNulty (2005), however, reveal more substantial results.

School effectiveness research in the 1970s assumed school leaders did indeed have a major impact on student outcomes and that the relationship was direct—in other words, principal behavior could lead directly to higher student achievement (Brookover, Beady, Flood, Schweitzer, \& Wisenbaker, 1979; Edmonds, 1979; Rutter, Maugham, Mortimore, Ouston, \& Smith, 1979, cited in Witziers, Bosker, \& Kruger, 2003). This line of research suggested that "principals should have high expectations of teachers and student achievement, supervise teachers, coordinate the curriculum, emphasize basic skills, and monitor student progress," (Witziers et al., 2003, p. 401). Bossert, Dwyer, Rowan and Lee (1982), however, in an article on the "instructional management" role of the principal, emphasized instructional leadership as an overall orientation toward student 
progress which is highly influenced by the particular school context. This understanding of instructional leadership suggested the effects of principal behavior may be more indirect and called for further studies to explore the difference. Over the next two and a half decades, researchers endeavored to operationalize instructional leadership as a topic of study and to examine the effects—-both direct and indirect—of instructional leadership on student achievement.

Andrews and Soder (1987) conducted a two-year study of Seattle schools investigating the role principals play in influencing student academic outcomes, especially for students the authors deemed "low achievers." Participants in the study included teachers from 33 Seattle elementary schools surveyed in the spring of 1984 and 1985. The researchers developed a survey questionnaire seeking teacher perceptions of their principals' instructional leadership relative to 18 specific behaviors, which constituted four key roles: (a) resource provider, (b) instructional resource, (c) communicator, and (d) visible presence.

From the teacher responses, the researchers ranked the principals $(n=33)$ according to their scores, with the 11 highest scorers in the first group, designated as "strong leaders," the middle 11 in a second group called "average leaders," and the lowest 11 in the third group called "weak leaders." The independent variable for the study, therefore, was whether the principal was ranked as a strong, average or weak instructional leader. The dependent variable was average student gains in normal curve equivalent scores on the reading and math sections of the California Achievement Test over a two-year period, with the students' 1982 and 1984 test performance serving as preand post-test data. The researchers only used data from students included in both the 
1982 and 1984 samples. The authors conducted an analysis of variance (ANOVA) to identify significant differences in student scores depending on whether their principal was ranked "strong," "average," or "weak" as an instructional leader.

Students scored significantly higher in strong-leader schools than those of students in average- or weak-leader schools. Likewise, minority students and students on free or reduced lunch experienced the greatest gains in strong-leader schools, while results were directionally inconsistent in average or weak schools.

Whereas Andrews and Soder's study (1987) focused on elementary principals, Blank (1987) investigated whether students achieved at significantly higher levels in urban high schools where principals engaged in higher levels of instructional leadership. The researcher conducted structured interviews with principals, district administrators and teachers from a stratified random sample of high schools $(n=16)$ from the 161 U.S. cities with populations greater than 100,000. Respondents ranked the level of instructional leadership in their school relative to six roles: (a) instructional improvement and innovation, (b) setting educational goals, (c) staff development, (d) district or community support, (e) involving staff in planning, and (f) exercising authority. The author coded responses to generate a scale of instructional leadership and then grouped schools according to whether their principal engaged in high-, medium- or low-levels of instructional leadership in each of the six roles. These groups constituted independent variables for the study. The dependent variable was measured in three forms of student achievement: average student scores in math and reading and average daily attendance. Analysis of variance (ANOVA) indicated that high levels of two leadership indicators - making decisions on curriculum issues and increasing academic learning 
time, were positively associated with high student scores in mathematics, even when controlling for percentage of low-income students. Reading scores were unaffected. Blank (1987) noted this finding was confirmed by previous research indicating that student learning in math was less susceptible to negative influences of variables in student homes and the media than was reading achievement. None of the areas of instructional leadership were associated with rates of student attendance.

By the end of the 1980s, studies such as those cited above established that instructional leadership was a multidimensional activity that had an indirect relationship to student achievement. These studies tended to be qualitative or correlational in nature and focused almost exclusively on instructional leadership as a personal trait or set of personal traits possessed by principals (and neglected contextual factors influencing instructional leadership). Furthermore, researchers had been unable to establish a model for measuring the relative contributions of the various behaviors that research suggested constituted instructional leadership as a whole.

Heck, Larsen, and Marcoulides (1990) addressed the limitations of previous studies. These authors developed a modeling procedure to investigate causal relationships between specific principal behaviors and student achievement and controlled for contextual variables that might affect outcomes. Based on earlier research into instructional leadership, Heck et al. (1990) proposed a predictive model of instructional leadership that suggested the way a principal governs the school impacts the school's climate and instructional organization, which in turn influences student achievement. Heck et al. (1990) tested this model by surveying a sample of teachers and principals on 22 instructional leadership activities in their schools, representing the three 
behavioral domains of the model (governance, school climate and instructional organization), and then measured which principal behaviors had the largest impact on student achievement.

The sample for the Heck et al. study (1990) included teachers $(n=168)$ and principals ( $n=30$ ) from 30 schools identified as consistently outperforming or underperforming with a "comparison band" of other schools based on student achievement on reading and math sections of the California Assessment Program (CAP) and that had the same principal for three consecutive years. The researchers selected comparison schools by controlling for socioeconomic status and language background within the population. The sample included both elementary and high schools. The independent variable for the study was whether the school was ranked high or low achieving compared with others. The dependent variable was average scores measuring the intensity of each instructional leadership behavior for the high-achieving schools and low-achieving schools. The researchers conducted $t$-tests to find significant differences in leadership behaviors between the high- and low-performing schools. The authors found significantly higher levels of instructional leadership in the higher-performing schools in all leadership behaviors except "involves parents in school program" and "develops school goals." Heck et al. (1990) then aggregated from the individual to school-level and conducted a second set of $t$-tests which indicated fewer significant differences, but still indicated consistently higher levels of instructional leadership in the high-achieving schools.

Heck et al. (1990) utilized structural equation modeling to test hypothesized causal relationships within their model of instructional leadership. The three domains of 
leadership behavior (governance, school instructional organization, and climate) served as independent variables. The dependent variable was student achievement over a threeyear period on the reading and math components of the California Achievement Program (CAP) test. The data confirmed the fit of Heck et al.'s (1990) model for instructional leadership as indicated by the coefficients of determination, goodness-of-fit index, and the Bentler and Bonett normed index. The root mean squared statistic indicated that the model explained almost all of the variance and covariance within the data. Heck et al. tested the model separately for principals and teachers and found that the model fit slightly less well with the principal data but still confirmed the overall structure of the model.

The data confirmed the authors' hypotheses (based on Bossert et al., 1982) that a principal's approach to school governance affects the school's climate and instructional organization, and that together governance, climate, and instructional organization impact student achievement. The study suggested that while involving parents in the school's instructional program had little impact on student achievement, principals in highperforming schools actively involved teachers in instructional decision-making at significantly greater levels. Approximately an equal number of principal behaviors related to climate and instructional organization significantly predicted student success, including expressing and enforcing high expectations for student academic and social behavior, establishing clear school-wide goals, encouraging the study of instructional strategies, communicating the school's progress with the community, and encouraging faculty enthusiasm and good morale. 
In a follow-up study using the same data set, Heck and Marcoulides (1990) explored whether the many contextual differences between elementary and high schools influenced principal leadership at each school level. Previous researchers revealed substantial contextual differences and suggested that because of the larger size and multitiered hierarchical structure of most high schools, instructional leadership might have a stronger influence on student outcomes at the elementary level. Heck and Marcoulides investigated whether the model developed and tested by Heck et al. (1990) fit differently for elementary schools versus high schools.

For this study, the independent variable was an elementary or high school and the dependent variable was the difference in parameter estimates from the structural equation model conducted by Heck et al. (1990) for the two groups. Tests for coefficient of determination, goodness of fit, Bentler \& Bonnett Normed Index and root mean squared residual all indicated no significant differences between elementary and high schools and confirmed that the model of instructional leadership fit across school levels. Likewise, the researchers found no significant relationships between latent variables (governance, climate, and instructional organization) across levels.

Heck and Marcoulides (1990) confirmed that principal behaviors have a significant indirect impact on student achievement regardless of whether the principal works in an elementary or secondary school, and Heck et al. (1990) found contextual factors and perceptions of teachers and principals regarding instructional leadership useful means of predicting student outcomes. Building on these studies, Krug (1992) explored how effectively both instructional leadership and "instructional climate" predict student outcomes and sought further confirmation as to the consistency of these 
predictive factors across grade levels. Krug also investigated how combinations of principal, teacher and student perceptions of instructional climate and leadership predicted student success.

The sample for Krug's (1992) study included teachers $(n=1,523)$, principals $(n=$ $81)$ and students $(n=9,415)$ in grades 3,6 , and 8 from 81 suburban schools in the Chicago area, representing elementary (75\%), middle (21\%) and high school (5\%) levels. Independent variables for the various tests included the extent of principal perceptions of their instructional leadership and other personal factors such as motivation, job satisfaction, etc., and teacher and student perceptions of their school's instructional climate. The dependent variable was average student achievement on the reading and mathematics sections of the 1990 Illinois Goal Assessment Program (IGAP) for grades three, six, and eight.

Principals in the sample responded to the Instructional Leadership Inventory (ILI), which included 48 Likert-type items measuring the frequency of principal behavior in five dimensions of instructional leadership: (a) defining mission, (b) managing curriculum and instruction, (c) supervising and supporting teaching, (d) monitoring student progress, and (e) promoting instructional climate. Principals also responded to the School Administrator Assessment Survey (SAAS), a self-report instrument measuring principal perceptions of their motivation, current job opportunities, school climate, job satisfaction and commitment. Previous studies validated both the ILI and SAAS. Teachers responded to the Instructional Climate Inventory (Form T), a version of the Instructional Leadership Inventory that paralleled the 48 -items on the ILI and measured the extent to which teachers perceived their principal engaging in instructional leadership 
activities and also included 60 items measuring school climate and teacher levels of commitment and job satisfaction. Students responded to another, 20 -item version of the Instructional Climate Inventory assessing school climate. Previous research studies had validated the instruments.

Previous research indicated that student perceptions of school climate decline progressively as students reach the early high school years, and then rise again slightly. To account for this, Krug (1992) converted student raw scores into standard scores based on grade-appropriate norms. Likewise, the researcher addressed difference in perceptions between elementary and high school teachers by adjusting scores accordingly based on norms identified in earlier studies. To ensure the generalizability of student and teacher aggregate scores from the surveys, Krug conducted an analysis of variance (ANOVA) comparing small random samples of both students and teachers to the mean scores for both groups as a whole and found no significant differences.

Following aggregation of scores, 30 psychological variables emerged for testing against student performance in each school. Following factor analysis, five factors emerged. The researcher conducted a step-wise correlation comparing the predictor variables with student achievement and excluded moderator variables. Findings indicated that principal self-perceptions regarding their instructional leadership significantly predicted student achievement. Teacher ratings did not correlate to student outcomes, suggesting that principal perceptions are a more important predictor. The combined teacher and student ratings for climate variables such as satisfaction, commitment, strength of climate and sense of accomplishment significantly correlated to student achievement. The strongest relationships emerged at the third-grade level, 
suggesting that instructional leadership, while holding a significant impact across grade levels, had the highest impact in the early grades.

Snyder and Ebmeir (1993) investigated variables that, in conjunction with principal leadership, predicted student achievement outcomes by utilizing path analysis. Snyder and Ebmeir hypothesized a path model suggesting that four contextual variables affecting principals, teachers, students and the school as a whole impacted both principal process variables and school variables relative to maintenance of motivational and values structures, adaptation to demands originating outside of the school, goal attainment and integration of all the various tasks necessary for student achievement. The authors next hypothesized that these principal-level and school-level process variables influenced a set of intermediate student outcomes (social and academic), intermediate staff outcomes, and collective intermediate outcomes for the entire school climate, which finally influenced specific outcome variables for student achievement and social and physical development.

To test this path model, the researchers selected 30 schools from volunteer school districts in Kansas and Missouri. The researcher administered questionnaires to teachers in all 30 schools and a random sample of students and parents. The questionnaire sought to measure 24 variables representing the context ("presage") and process variables of the model. These 24 variables served as independent variables for the tests used in the study. Intermediate outcome measures such as student self-concept and self-reliance and teacher job satisfaction and morale served as dependent variables measured by the survey questionnaires. The path analysis utilized correlation and multiple regression techniques to determine the direct and indirect effects and relationships among variables, which the 
researchers then compared to the hypothesized paths of the model to confirm or disconfirm its fit, and trimmed the path models accordingly.

Snyder and Ebmeir's study (1993) found, among other things, that while principal behaviors directly impacted teacher outcomes and teacher perceptions, they did not directly affect student outcomes. Principal behaviors, however, did have an indirect influence on student norms, students' sense of academic futility and on parent satisfaction. In this way, Snyder and Ebmeir confirmed previous research indicating primarily indirect principal effects on student achievement, and confirmed Heck et al.'s (1990) assertion that these effects were not just relational but causal as well.

Heck (1993) researched the interplay of contextual variables and principal behavior on student outcomes in a study based in Singapore. The sample for Heck's study included elementary and secondary teachers $(n=156)$ from 26 randomly-selected schools. Participants responded to a 42-item Likert-type survey assessing teacher perceptions of teacher-principal interactions, school governance and instructional structures, climate and culture. A confirmatory factor analysis narrowed the study down to 21 observable leadership behaviors that aligned with the three factors identified by Heck et al.'s (1990) model of instructional leadership domains: governance, climate and instructional organization. Thus Heck's (1993) questionnaire proved a valid instrument for measuring instructional leadership and school contextual variables according to the Heck et al. (1990) model.

In the first portion of Heck's (1993) study, the researcher treated contextual factors such as school level, size, type and level of teacher education and experience as independent variables and conducted a multivariate analysis of variance (MANOVA) to 
determine whether these factors predicted significant differences in teacher perceptions of governance structure, school climate/culture and instructional organization (which served as dependent variables). Heck found no significant differences, indicating that contextual variables did not influence perceptions of the leadership processes under investigation. Next, the researcher conducted a discriminant-function analysis to determine whether teacher responses to the questionnaire could predict whether the teacher worked at a high-, medium- or low-performing school. High-achieving schools had more teachers with high-expectations for student achievement, and principals who actively involved teachers in instructional decision-making, and who had high perceptions of the school's climate and culture. Heck (1993) further confirmed that principals significantly impact teacher perceptions, which then predict higher levels of student achievement.

Pounder, Ogawa and Adams (1995) broadened the understanding of "leadership" as not just something that principals do, but an organizational quality that schools may possess to greater or lesser degrees and which impacts organizational performance. Pounder et al. investigated the relationship between the presence of leadership among teachers and secretaries as well as the principal and its impact on four measures of student outcomes. The researchers conducted a path analysis to determine how Parson's (cited in Pounder et al., 1995) four dimensions of effective organizations (adaptation, goal achievement, integration, and latency) impact student outcomes.

The stratified random sample included employees $(n=1,061)$ from one large suburban district in the intermountain west representing different employee roles at each of 60 schools, including all secondary schools and a random selection of elementary schools. Since some schools failed to return enough usable surveys, the final sample 
included 57 schools. Respondents included teachers (78\%), plus secretaries, custodians, counselors and administrators from each school site. Participants responded to a survey featuring Likert-type items designed to measure perceived levels of organizational leadership exercised by various individuals or groups within the school (principal, school secretary, staff members acting alone, collective groups of faculty members and patrons from the school community). The level of leadership within each group served as the independent variables. The four dimensions of leadership (adaptation, goal achievement, integration and latency) served as intermediate variables, and student outcomes as measured by average student test scores, student attendance rates and staff turnover over a three-year period served as dependent variables.

Simple descriptive statistics indicated that employees perceived the principal to have the greatest level of influence over the school. A series of multiple-regression analyses between the independent, intermediate and dependent variables served to confirm or disconfirm pathways of influence within the school community and with specific outcomes. In terms of principal effectiveness, Pounder et al. (1995) found that principals had a significant influence over the intermediate variable of "latency" (which has to do with school culture), and latency correlated with higher levels of perceived effectiveness and low rates of faculty/staff turnover. In terms of total organizational leadership, the focus of the Pounder et al. study, the degree of total organizational leadership impacted the school's sense of goal-orientation, which in turn highly correlated with increased levels of student achievement.

Witziers, Bosker, and Kruger (2003) summarized previous research on principal effects by conducting a quantitative meta-analysis. Witziers et al. (2003) collected 
quantitative studies $(n=37)$ on principal effects and student achievement from 1986 (the year that marked the first sophisticated path analysis and causation models) to 1996. To focus the meta-analysis, the researchers used only studies that had a clear operational conceptualization of instructional leadership and specific and valid measures of student achievement and that did not focus on other outcomes. Additionally, the authors used data from the International Association for the Evaluation of Educational Achievement (IEEA), a literacy assessment conducted in 25 countries to create separate cases for each country to compare principal effects from one country to the next.

Witziers et al. (2003) conducted three distinct meta-analyses for their study. For the first analysis, the researchers reviewed results of all the studies simultaneously, averaging effect sizes when studies had measured separate sub-domains of instructional leadership. A second meta-analysis focused only on studies that had a one-dimensional conceptualization of instructional leadership. Finally, the third meta-analysis involved a series of analyses on each of the sub-dimensions featured in the studies. To create a consistent form of data, the researchers converted results from all studies to a common correlation form using Fisher's $\mathrm{Z}$ transformation of the correlation coefficient. In all cases, the researchers conducted each analysis twice, removing outliers the second time through, in order to check the robustness of the findings. These meta-analyses investigated which factors explained the variation in effect sizes among the collected studies.

Witziers et al.'s (2003) first meta-analysis confirmed that principal behavior did indeed have a positive and significant effect on student achievement, but with relatively small effect sizes (.11 for studies in elementary schools in the United States, based on 
respondents other than the principal and using multi-level modeling techniques).

Moreover, the researchers found no significant effects for studies in secondary schools. In the subsequent meta-analyses, Witziers et al. (2003) found that one-dimensional measures of principal leadership failed to significantly equate with student outcomes, and studies that focused on sub-dimensions of leadership tended to have small effect sizes. Four specific leadership behaviors did have significant effects with somewhat larger effect sizes: supervision and evaluation, monitoring, visibility and defining and communicating mission (the last having the largest effect size, and thus, the behavior with the largest real impact on student achievement). In their study of moderating variables' impact on student outcomes when paired with principal behaviors, the researchers found only a few significant cases, and sometimes in inconsistent directions. For example, being a secondary school was a moderating variable that had a significant impact on outcomes - it meant that principal effect sizes were lower. In summary, Wiztiers et al. (2003) confirmed many previous research discoveries of indirect principal effects on student achievement, with small overall effect sizes.

Marzano, Waters, and McNulty (2005), however, addressed some of the limitations in Witziers et al.'s study and provided a much more comprehensive overview of previous research on principal impacts on student achievement. Marzano et. al (2005) expressed a concern that previous meta-analyses had, on the one hand, not included large enough samples of studies, and that the other studies may have included data from schools outside the United States too culturally or demographically different for reliable comparison. By searching for articles, dissertations and research reports between 1970 and 2003, the authors collected 5,000 studies on the impact of principal behavior on 
student achievement. To focus their meta-analysis, Marzano et al. limited their investigation to studies that met the following criteria: (a) involved schools spanning kindergarten through twelfth grade, (b) included only schools in the U.S. or schools that closely resembled those in the U.S. (such as in Canada or the UK), (c) examined direct or indirect effects of principal activity on student achievement, (d) measured student achievement in terms of a standardized achievement test or state assessment (or a composite index from one or both), and (e) computed and reported effect size. With these boundaries for their meta-analysis established, Marzano et al. identified studies ( $n=$ 69) representing 2,802 schools, approximately 14,000 teachers and 1.4 million students between the years 1978 and 2001.

Marzano et al. (2005) sought to improve on Witziers et al.'s (2003) meta-analysis in three ways: a) by including only schools from the U.S. or schools very similar to those in the U.S., b) by utilizing a process for computing outlier data, and c) by using methods to correct for attenuation, or the gradual weakening of correlational coefficients due to the imprecise nature of many of the measurements used in the collected studies.

After conducting their meta-analysis, Marzano et al. (2005) found a substantially stronger relationship $(r=.25)$ between instructional leadership and student achievement than previous meta-analyses had indicated. More precisely, the researchers found that one standard deviation of increase in instructional leadership equated to a ten-point percentile gain in student achievement. Through a factor analysis, Marzano et al. found 21 specific principal behaviors significantly related to increases in student performance. The strongest relationships emerged in the area of situational awareness, using information to address current and potential school problems $(r=.33)$, followed by 
flexibility, adapting leadership behavior as needs present themselves $(r=.28)$, and then managing discipline, monitoring and evaluating and outreach to the community and stakeholders $(r=.27)$.

Additionally, Marzano et al. (2005) found that the effect of specific behaviors was greatly influenced by the order of magnitude of change the behavior implied. Drawing on previous literature, Marzano et al. grouped change initiatives into two categories, firstand second-order change. First-order change initiatives are incremental and are intended to simply apply existing norms and paradigms to new problems in the organization. Second-order change situations, on the other hand, involve a wholesale transformation of beliefs, practices and norms of behavior. All 21 principal leadership behaviors correlated with positive outcomes in first-order change situations. Marzano et al. found that only seven of the 21 behaviors correlated to outcomes in second-order change situations: (a) knowledge of curriculum, instruction, and assessment, (b) optimizer, (c) intellectual stimulation, (d) change agent, (e) monitoring/evaluation, (f) flexibility, and (g) the capability of expressing one's ideas and beliefs in a coherent, meaningful way. Thus, Marzano et al. (2005) emphasized the importance of principals carefully studying the context of their particular schools and emphasizing instructional behaviors that are appropriate for the order and magnitude of change the situation presents.

In sum, principals play a key role in helping schools achieve the student outcomes goals of state and federal accountability initiatives. Researchers for three decades, culminating with the work of Marzano et al. (2005) have progressively established a connection between principal behavior and student outcomes, though this relationship may be indirect and mediated by other variables related to school culture - the values, 
beliefs and assumptions that guide daily decision making and which are largely unchanged despite the onslaught of school reform efforts. The next section explores school culture and the change-resistance that seems to accompany it, and suggests the role principals might play in transforming school culture.

\section{Resistance to Change: Confronting the Culture of Schools}

This section explores literature on schools as systems and the culture of autonomy and isolation within schools, which stands in stark contrast to the culture of openness, trust and collaborative inquiry that one would expect to find in a restructuring school. Research shows that school administrators do play a critical role in shaping school culture and can engage in behaviors that intentionally promote a more trusting, collaborative and inquiry-oriented work environment for students and teachers.

Cusick (1992) did not set out to explore the issue of resistance to change in schools. Utilizing a systems approach, he intended to describe the nature and logic of the many overlapping collectivities that make up the school system itself. Cusick examined descriptive studies from two decades of qualitative research focusing on various subgroups of individuals — students, teachers, administrators, outside parties, and government and reform groups - and how the inter-dynamics of these various groups create a system with its own internal logic and rules of order. Cusick found that the overarching theme of the entire educational system could be described as a need for control. This need emerges from the many uncontrollable variables that are involved in the educational process - student innate abilities, socio-economic barriers to learning, limited resources, the many competing political and social values that students bring to school with them and that lead to a plethora of pressures from groups outside the formal 
system, and above all the tendency of students to form their own groups and operate according to value systems that may be entirely at odds with the values the school wants them to learn and operate by.

The public provides a general consensus of what they want from schools—-some degree of common literacy and numeracy, social skills and work ethic, and some mechanism by which to judge whether the schools have achieved this, and they want it with a minimum amount of economic commitment. Meanwhile, the students have an agenda all their own, one that is primarily social and that reflects and reinforces the diversity that competes with the uniformity implied by a common set of curricular and behavioral standards the school is required to impose. The primary arena in which this drama takes place is the individual classroom, and the teacher finds that the tension between the uncontrollable variables and the high, if vague, expectations of the system leads to a need for imposing the maximum amount of control that the students and outside forces will tolerate. "Because much of [the students'] behavior falls outside the school's definitions of appropriateness, control is the central problem" (Cusick, 1992, p. 68):

It is the teachers' problem, and they solve it by placing themselves in the center of the class and using their role, liberally mixed with their values, backgrounds, and personalities to manage both the students and the flow of events. For teachers, the problem is to impose the narrow on the broad. (p. 69)

Because the teacher must be concerned primarily with the control of events in his or her classroom, teachers begin to work in isolation from one another. "A professional distance [between teachers] is maintained," Cusick wrote. "Each [teacher] has the problem of imposing the school's definition of reality onto students. This is a personal problem and teachers solve it personally" (p. 96). 
This professional isolation not only shapes the relationships of teachers with one another, but of teachers and their administrators. Principals need teachers to control the events within their classrooms and teachers need principals to control as many variables outside the classroom as possible to keep them from spilling through the classroom door. The teachers' primary expectation for principals is the smooth, orderly operation of the school's day-to-day affairs, rigorous discipline of unruly students, and protection from aggressive parents: "For orderliness, the teachers rely on the principal and they judge him [sic] on whether he provides it. If he does, they like him, even when they don't like him; if he doesn't they don't like him, even when they do" (Cusick, p. 96).

Cusick (1992) carefully emphasized that he was making no value judgment on the educational system for its stress on control, only describing the phenomena that emerged so clearly from descriptive studies. He also acknowledged that control "may be an unsatisfying term; it is not meant to imply a heavy-handedness or severity":

Instead, I mean an acceptance of common norms, graceful relations, and easy coordination of myriad overlapping events. This is by nature problematic in schools that are crowded, dense, and busy, particularly those that contain many students who resist the school's definitions of appropriate behavior. (p. 96)

The principal faces a daunting task: managing the crushing expectations of parents, community leaders, reform organizations, governments and the needs and demands of students and teachers with limited time and resources. Meanwhile, the principal has to personally maintain an idea of the school as a unified, coherent organization. The result for the principal, like the teacher, is a carefully nuanced emphasis on control. "Operationalized, school administration is the taking of one of the innumerable elements that is or threatens to be out of kilter with the others and putting it back into the routine, or even fitting something new into the routine" (Cusick, 1992, p. 
124). Cusick argued that the principals were completely justified in their obsession with control, because ultimately that is the system's expectation for the principal's role, and that the system itself would make no sense otherwise, by its own nature and logic. If principals ...

eased off on maintenance and control...events would begin to back into one another, rumors of "lack of control at school" would spread and the administrator would be fired. A new administrator would be brought in and told that she or he was to "get things under control. (pp. 124-125)

Such a situation leads principals to be guarded in their emotions and decision-making processes and to be suspicious of influences from outside the school, despite the expectation that they also be public, welcoming and engaging personalities within the school community.

Cusick (1992) found that another key feature was the systemic tolerance for high degrees of individual freedom. Teachers, especially, value autonomy because it allows wide decision-making authority within the classroom and enhanced control. The system also acknowledges that there is a limit to the level of control that students will tolerate, and so decisions are made with that in mind:

Students decide how and to what degree they will participate in class. Teachers decide how and to what degree they will comply with administrators. Each reserves the right to alter the definition of the situation and make judgments from his or her own perspective. This right of individuals to behave as they wish is an important characteristic of the system. (p. 97)

This recognition that teachers and students will only accept so much control reinforces the principal's need to control carefully forces that are actually within his or her realm of influence.

In the final part of Cusick's (1992) analysis, he explored how the overwhelming emphasis on control interacted with the inevitable push for reform and change within the 
educational system. He found a relatively high tolerance for reform efforts in the system, but such changes were channeled and institutionalized in a way that reinforced and supported the internal nature and logic of the system itself. This resulted from the system's tendency toward bureaucracy and specialization. To maintain maximum control of the many variables intruding on the educational process, schools established bureaucratic systems, such as committees, programs, specialists or even experimental or magnet schools to address a particular group or individual needs or concerns.

These bureaucratic systems provided an orderly and predictable method for handling problems that threatened to impose substantial changes on the normal operations of the system. So, for example, the reform-minded effort to provide higherquality educational opportunities for students with disabilities, ensconced with the Individuals with Disabilities in Education Act (IDEA) of 1975, lead to specialized teachers who worked with students in special classrooms with specialized budgets designed to meet the needs of children with special needs, thus minimizing any real changes to the way that "normal" classes are taught or how schools as a whole operate. The same would be true for gifted and talented students, or for students with specialized vocational interests. The movement for charter and magnet schools that specialized in curricula for the arts or for skilled trades were particularly vivid examples of the expense the educational system will go to in order to respond to the demands for change by offering a supplementary program that will fit within the structure of the system while maintaining a maximum level of control and orderliness to the system's stability.

Cusick's (1992) description sheds light on the difficulty of change with schools. Consider the relationship between teachers and administrators in Cusick's study. The 
system's emphasis on control reinforces the tendency of teachers to work in isolation from one another, and to expect the principal to emphasize orderliness and stability. For the teacher who thinks he or she knows what works in his or her classroom, the idea of learning from another teacher, perhaps one with less experience or who teaches in a different grade level or subject area, does not fit the logic of the system. Likewise, a principal who emphasizes instructional innovation and group decision-making may be viewed as neglecting his or her primary responsibility toward control of variables outside the classroom, and will be violating the understood assumption that what happens within the classroom is the teacher's responsibility. Collaborative decision-making is risky, time consuming and unpredictable, qualities that threaten stability, order and control. Furthermore, action research and professional inquiry may reveal ideas or practices that are at odds with the school's present methods for maintaining control or expose values, ideas and assumptions that are in conflict with best practices.

To sum up the argument made so far, any discussion of how schools can raise student achievement and become centers of inquiry and workplaces that value questioning, doubt, and experimentation will have to take into account this overwhelming tendency within the educational system toward control. When genuine efforts to make schools the center of change are successful, what happens to this control-orientation among teachers and administrators? Does it fade away altogether, suggesting that the very nature and logic of the school has changed, or does it remain in some altered or mitigated way? Such questions imply a change in the normative thinking of teachers and administrators, rather than the typical structural changes invoked by most educational reform efforts. 
Cusick's (1992) conception of schools as change-resistant, control-obsessed fortresses braced against an onslaught of external pressures stands in contrast to Sirotnik's (1989) argument that as long as schools are the targets of change, then schools will continue to struggle to solve problems in new and creative ways. Sirotnik argued that schools must become centers of inquiry-workplaces that embrace continuous learning and the testing of theories and their efficacy in the particular context of an individual classroom and school. According to Sirotnik, educational reformers have traditionally overemphasized positivist approaches to knowing, such as "explanations derived from experimental, quasi-experimental, and correlation studies in the tradition of the scientific or hypothetico-deductive methods" (Sirotnik, 1989, p. 91). Sirotnik viewed this kind of narrowly defined "empiricism" as fostering a kind of artificial barrier between "knowers . . . and what is to be known" and seeking predictable, "law-like relationships between variables" (p. 92). As a result, for a century "experts" (scholars and researchers usually specializing in experimental design methodologies) figured out the answers to improved student achievement; more "experts" (this time usually in the form of educational bureaucrats at the state and federal level) translated these answers into policies, and then the local districts imposed these new policies on the teachers expected to successfully implement them. Teachers - the actual people who work with students every day — were thus completely removed from the real work of learning about learning.

Bridging the gap between theory and practice, according to Sirotnik (1989), means using a phenomenological design. Teachers must become the central researchers in the process of learning about learning, and in particular learning about how the 
individual students in his or her individual classroom learn most successfully. This might imply experimental design, but it suggests a broad range of qualitative ways of "knowing" as well, including "observation and case study—ethnography, ethnomethodology, symbolic interactionism, grounded theory and so on” (p. 94). In such a way, teachers would be equipped to test the extent to which their values, beliefs and assumptions actually guide the daily activities in their classrooms and schools, a key component of developing theories of practice:

Through the process of rational discourse, critical-dialectic methods require of those to whom knowledge and action matter most that they tease out tacit beliefs, values, and human interests - both their own and those embedded in the production of knowledge in the first place. The idea of producing knowledge one place and then installing it for use in another place is an alien concept in the world of critical inquiry. Regardless of where and how knowledge is generated, criticaldialectical methods demand that it be 're-known' in the context of values-based human activity - a concept of critical knowing in action or 'critical phenomenology,' if you will. (Sirotnik, 1989, pp. 98-99)

If educators engaged in such approaches to learning about learning, Sirotnik argued, schools would soon become centers of inquiry, and by extension centers of change.

Keedy (2005) confirmed Sirotnik's argument that a deep schism existed between theory and practice in the professional work of educators, and traced its historical roots. Keedy (2005) outlined how this historic lack of reflection among educators emerged as a result of the Positivist and Technical-Rationality movements in the late $19^{\text {th }}$ and early $20^{\text {th }}$ century and documented how these philosophies contributed to an ever-deepening schism between theory and practice, both in the training of education administrators and also in the schools where they serve. That schools are not places of critical self-reflection is therefore not a new problem, nor are some of the possible solutions to the theory-practice schism new. However, the emphasis on structural changes to improve schools has 
distracted teachers and principals from the real work of questioning normative thinking that is necessary for healing the theory-practice divide.

Keedy and Achilles (1997) argued that because of this historical schism between theory and practice present-day school reform efforts would fail as long as they focused exclusively on structural changes and neglected the deeper issue of normative thinking on the part of teachers and principals. The collective values, beliefs and assumptions of teachers and administrators significantly mediated the long-term effectiveness of structural changes in schools, such as site-based management and reforms in teacher professional development and instructional practice. The authors argued changing relationships among education stakeholders should be the key litmus test for the effectiveness of school restructuring efforts:

Classrooms in the USA are, generally, boring places; the modal number of questions that students ask of their teachers per high school class period is two. Sizer puts it bluntly: teachers, rather than students "do the work"... [Students] are not engaged in the material and do not have to make their own meanings. (Keedy \& Achilles, 1997, p. 2)

Keedy and Achilles (1997) contrasted this kind of student-teacher relationship with the kinds of relationships one would expect to find in a restructured school, characterized by "compassion, cooperative effort, student mindfulness and mutual respect for articulate and diverse positions on crucial issues" (p. 3). Likewise, the authors argued that relationships between teachers and teachers and between teachers and principals are also transformed in a genuinely restructuring school, taking on more collaborative approaches to decision-making and marked by trust, openness and mutual support for self-inquiry and self-development. This kind of relationship stands in stark contrast to the kinds of teacher-teacher and teacher-principal relationships described by Cusick 
(1992), which are focused on isolation and could be described as "you do your job and I'll do mine."

Keedy and Achilles (1997) cited a number of research studies indicating that the basic relationships among teachers and between teachers and their administrators was relatively unchanged despite many efforts to restructure schools. The norms for decisionmaking and the daily operation of schools have remained the same, and therefore relationships among school stakeholders remain characterized by isolation, independence (as opposed to interdependence), and control. Keedy and Achilles argued that normative thinking about teachers and principals must first be transformed for structural changes to be able to fundamentally impact relationships within the school organization. This is new territory for most schools:

Normative thinking requires staffs to reflect critically about their schools as highly professional workplaces where teachers, students and principals form thoughtful, caring relationships. US Schools, however, have not been reflective places - despite Dewey's call for use of inquiry. This historical lack of reflection appears to be still with us. (Keedy \& Achilles, 1997, p. 5)

Other recent empirical studies document the difficulty of creating sustainable school reform efforts. Datnow (2005) and Hargreaves and Goodson (2006) did not examine the issue of principal reflection per se, but their research confirms the obstacles school reformers face and the relative lack of change over time. Datnow (2005) investigated six comprehensive school reform (CSR) models in 13 schools to examine the sustainability of reform efforts under changing contextual conditions. Previous literature documented the difficulty of implementation of CSR models in a variety of school contexts, but few studies examined the dynamics of CSR implementation over extended periods of time or the impact of changing district and state contexts on CSR success. 
The sample included 13 elementary schools from a culturally and linguistically diverse urban U.S. school district. The research team collected data over a four-year period, from 1996 to 2000 . The 13 schools attempted to implement six different CSR models, including Success for All, Modern Red School, the Audrey Cohen College System of Education, Core Knowledge, the Coalition for Essential Schools, and the Comer School Development Program. The sample included at least two schools implementing each model, with three schools implementing Success for All. These reform models range from highly structured curricula and instructional methods to broad, locally-developed approaches. The researchers conducted over 300 interviews with teachers, administrators, students, parents and design team consultants. For this article, Datnow (2005) analyzed transcripts of school staff and reviewed detailed case studies of each school developed by the research team. A grounded theory approach guided Datnow's analysis of the data and the researcher developed matrices to highlight withinand across-schools patterns.

Datnow found that after three years six of the 13 schools in the study had abandoned their CSR model, and two other schools were still nominally implementing their models, but at a relatively low-level of intensity. Five of the 13 schools were continuing to implement their reform model at moderate to high levels of intensity. The researcher described a variety of state and district-level factors that negatively impacted implementation of the CSR models. District mandates that directed the implementation of reform models were a significant inhibitor of program success. CSR models that persisted in the schools were those that supported or at least did not conflict with district demands. A second implication of the study suggested that CSR efforts were often far 
more expensive and time-consuming than stakeholders originally anticipated, and that considerably influenced implementation. Finally, Datnow argued that state-mandated, high-stakes accountability testing had a negative impact on CSR efforts because the standardization of curricula and the enormous pressures for schools to produce measurable outcomes left little room for innovation and little time and resources for the kind of collaborative process that some of the CSR models suggested.

Hargreaves and Goodson (2006) reported on a study of eight secondary schools and their experiences with both continuity and change over a thirty-year period. Their data were part of a larger long-term ethnographic study of schools located in Canada and the United States and representing a variety of communities, structures and school cultures. Most of the data from the Hargreaves and Goodson study came from intensive interviews with three cohorts of teachers who had worked in the schools in the 1970s, 1980s, and 1990s. The sample was random and stratified to provide a balanced representation and a subset of teachers belonged to two or all three of the cohorts because of their long tenure at the schools. The researchers also interviewed principals and at least one assistant principal from the 1980s and 1990s cohorts.

The researchers conducted semi-structured interviews with the participants and questioned teachers to assess their perspectives on a wide variety of issues: (a) motivation and patterns toward entry teaching, (b) working relationships with colleagues, (c) perspectives on and espoused practices concerning teaching, learning and the nature of their students, (d) teacher careers and career stages, (e) teacher understanding of and metaphors for the culture of their schools, (f) past and present experiences of internally and externally imposed change, (g) and the connection between teachers' experience of 
work and change to their lives outside of school. The researchers triangulated data from the interviews with school observations, documentary analysis and data collected at the school district level.

Data analysis was multi-staged. The researchers collaboratively wrote extensive school case studies of more than 100 pages and used a grounded theory analysis approach to identify emerging themes and patterns on the school level. After an initial set of nine themes emerged, the researchers crystallized the data into five main change forces that impacted the schools during this 30 -year period, including waves of policy reform, changes in leadership, changing teacher demographics, shifting student and community demographics and changing patterns of relations among schools. The researchers conducted a cross-case analysis to identify patterns in how teachers at the schools responded to these change forces over time. Hargreaves and Goodson (2006) found that three broad periods of change characterized the reforms and responses to reforms experienced by the schools under study: a period of optimism and innovation in the wake of the baby boom and broad increases in public funding for education (up to the mid-tolate 1970s); a period of complexity and contradiction, marked by changing demographics and cultural values, as well as widespread doubt in the efficacy of public schools (late 1970s to mid 1990s), and finally our current period of standardization and marketization, characterized by accountability measures, standardized assessment of student achievement, and increasing pressure for market-based competition for public schools.

Each of the five change forces and three periods of reform had a discernible impact on the schools under study, but the researchers concluded that all the schools with traditional structures persisted largely intact with little fundamental reorganization of 
curriculum, teaching or learning. In fact, Hargreaves and Goodson (2006) concluded that the era of standardization had reinforced the traditional paradigms and structures of schooling in these schools in many ways...

... through reaffirming the status of high-status subjects, through increased demands for content coverage, and through reinstating and reinforcing the power of departmental decision making. Other parts of the reform movement such as portfolio assessments, mentoring programs for students, and more personalized career counseling were squeezed to the side by these high-stakes imperatives. (p. 32)

The authors found that the four schools in the study that actively resisted these efforts at standardization in order to maintain their nontraditional structures or approaches to curriculum or instruction had become marginalized within their districts and in one way or other succumbed to the homogenization that accompanied this new era of reform. Hargreaves and Goodson concluded that ultimately it is standardization itself that has become the "ultimate enemy of enduring innovation and sustainable learning communities" (p. 32).

In sum, despite the difficulty of meaningful, sustainable school change efforts and the lack of professional reflection on the part of principals, a number of empirical studies over the last two decades indicate that school principals can, in fact, have a major impact on the culture of schools and relationships among teachers and especially between the administrator and teacher leaders.

Principal Effects on School Culture, Teacher Perceptions and Teacher Behavior While Hallinger and Heck (1998) confirmed that principal influences on student achievement are indirect, a number of empirical studies shows how these indirect effects function to influence school culture and teacher perceptions and behavior. This section describes such principal effects. 
Friedkin and Slater (1994) studied the effects of principal centrality to the social networks of schools on student performance. Using a network analysis approach, the authors studied the social networks in 17 California elementary schools, hypothesizing a positive relationship between a principal's centrality in the school's social network and average student scores over a four-year period on three sections of the state's achievement test.

Participant schools $(n=17)$ included students from a variety of socioeconomic backgrounds and principals whose tenure had lasted at least four years. The independent variables for the study were the principal's centrality to the school's social network and the social cohesion of the school staff. The dependent variable was student performance based on four-year averages of the standardized test scores from three sections of the California Assessment Program (CAP) exam administered in grades three and six: reading, language and mathematics. The researchers administered a questionnaire to principals and teachers in each school that asked the respondents to check the names of other staff members in three columns representing the names of people (a) with whom the respondent regularly discusses events or issues that arise within the school, (b) whom the respondent turns to for professional advice, and (c) whom the respondent considers a close personal friend.

Friedkin and Slater measured the principals' centrality to the social network by the number of teachers who listed the principal as a person with whom he or she discussed professional problems or turned to for professional advice. Social cohesion was measured by the density of network ties among staff regarding discussion, advice or 
friendship. The researchers utilized Kendall's tau to analyze associations among the rank-ordered data.

The study revealed that there was a significant association between a principal's centrality to the advice network within the school and student performance $(\tau=.459, p<$ .01 ), while the principal's role in the friendship network had no bearing on student performance. Density of professional ties among teachers, based on discussion or advice, had a positive association with school performance $(\tau=.356, p<.05 ; \tau=.485, p<.01)$, while density of friendship ties had a marginal negative association with school performance $(\tau=-.252, \mathrm{p}<.10)$. The researchers concluded that when a principal occupies a central role in the social network of a school, and when he or she is viewed as a source of professional advice and authority, there is a strong corresponding association with student achievement. These associations held strong even when the researchers controlled for community environment and school structure. In particular, Friedkin and Slater (1994) noted that the data suggested two key dimensions to principal advice centrality: (a) accessibility and attentiveness to matters of concern to teachers, and (b) collaborative problem solving and decision making on instructional issues in a context of mutual respect.

Pursuing a more qualitative approach, Short (1994) investigated principal behavior in schools identified as empowering teacher leadership through self-managed work groups. Short assumed that empowerment through self-managed teacher teams addressed the culture of isolation and control described by Lortie (cited in Short, 1994), Cusick (1992) and others and encourage creativity, innovation and collaborative inquiry. 
Participants for this study consisted of a purposive sample of students, teachers and administrators at four middle schools (two urban, two suburban) from a Mid-Atlantic state identified by university professors, public school personnel, and school administrators as utilizing highly-effective interdisciplinary instructional teams. The researcher visited ten schools recommended by the panel of experts and narrowed the study to four that featured teams that paralleled the key features of self-managed teams from the literature: a) autonomous functioning and b) self-direction. The researcher visited each school three days per month for six months and conducted observations of the school's general operations, the behaviors of principals and of instructional teams during their interactions. The author "triangulated" data by also conducting interviews on alternating months with the school principals and teacher teams and by conducting three focus-group interviews with samples of students from the interdisciplinary teams. Finally, the researcher analyzed school documents.

A team of researchers coded field notes from the observations according to role behaviors, attitudes and knowledge of the principal relative to developing the instructional team. The researchers content-analyzed these results to generate questions for loosely-structured interviews and to identify emerging constructs using a process of analytic deduction, recording data by number and type of response and then, as a means of strengthening the trustworthiness of the data, by seeking alternative or rival explanations for the emerging construct.

Short (1994) found that principals in schools with high-functioning selfmanagement teams played a key role in team success. A central principal behavior in these schools was encouraging and facilitating teacher reflection by posing problems to 
teams designed to stimulate teacher collaboration and problem solving. This strategy assumed that the principal did not have all the answers and placed responsibility for key decisions with teachers. Short found a secondary theme in the principal's role in facilitating the goal-orientation of teacher teams. In the school Short deemed the most effective at using self-management teams, the principal would often attend team meetings and simply ask about the progress of certain students or instructional initiatives, which assisted the teachers in following through on their goals and maintaining focus. Principals also encouraged teams to be both self-critical of their performance and to celebrate and publicize team successes.

Along similar lines to Short's study (1994), Blase and Blase (1994) investigated teacher perceptions of principals they deemed successful facilitators of shared decision making and the effects of such empowering principal behaviors. The researchers administered an open-ended questionnaire to teachers $(\mathrm{N}=285)$ in 11 Georgia schools, including elementary, middle, and high schools, all of which were charter members of the League of Professional Schools. The League provided training and action research protocols for schools in the process of implementing shared governance protocols beginning in 1990. Blase and Blase chose schools for the study based on recommendations from League staff members as to which schools demonstrated the highest levels of implementation of shared decision making.

Teachers described in writing strategies used by their principals to promote teacher empowerment, and described the effects these strategies had on them. Blase and Blase analyzed the teacher responses using a constant comparative analysis technique to identify emerging categories of principal strategies and effects. The researchers found 
two key strategies used by principals that encouraged empowerment: building trust and creating enabling structures of school governance and decision-making. Other strategies used by these successful principals included the following: (a) being personally supportive, (b) encouraging autonomy and innovation, (c) permitting risk-taking behaviors on the part of teachers and minimizing threats as a result of failure, (d) offering rewards, and (e) engaging in collaborative problem solving. Blase and Blase emphasized that teachers reported all 11 principals utilized these strategies and that the teachers reported that principal leadership, as defined in terms of the strategies, was by the far the largest contributor to the teacher empowerment.

Teachers in the study reported that the strategies used by shared-governance principals affected three dimensions of their sense of empowerment, including the affective dimension (teacher satisfaction, motivation, esteem, confidence, security, sense of inclusion, and identification with colleagues as a unified group), the classroom dimension (including innovation, creativity, reflection, autonomy, individualization of instruction, professional growth and classroom efficacy), and the school-wide dimension (expression, ownership, commitment, sense of team, and school-wide efficacy). Blase and Blase stressed that successful, shared-governance principals had clear visions and assumptions about collaborative leadership, but primarily saw themselves as facilitators of a process of school-wide teacher empowerment.

In a follow-up to the Blase and Blase (1994) study, Blase, Blase, Anderson, and Dungan (1995) conducted in-depth interviews with eight principals identified by the League of Professional School as having a history of successfully facilitating shared- 
governance structures in their Georgia schools to explore their backgrounds, motivations, assumptions and practices related to effective collaborative leadership.

Staff leaders in the League of Professional Schools, personnel for the Georgia State Department of Education, and educational administration faculty from the University of Georgia reviewed a pool of 45 possible participants and made recommendations on the eight principals who most exemplified empowerment of teachers through shared decision making. The sample included both men and women and represented a variety of experience levels. Their schools were urban, suburban, and rural and included elementary, middle, and high schools.

Over a six-month period, Blase et al. conducted open-ended interviews with each of the participant principals utilizing a protocol of questions that explored the following issues: (a) personal development as democratic leaders over their professional careers, (b) purposes/goals of democratic leadership, (c) strategies/techniques used to enact democratic leadership, (d) major problems and crises confronted as democratic leaders, (e) major sources of stress/failure and gratification/success, (f) values/ethical issues related to democratic leadership, (g) impact of actions on others, (h) relationships with school stakeholders and how such relationships hindered/helped democratic leadership, and (i) projections of future democratic leadership and democratic schooling. The researchers audiotaped all interviews and then after reviewing transcripts conducted follow-up interviews for completeness and clarification of the data. The researchers analyzed data according to the guidelines of grounded theory and constant comparative analysis. The researchers clustered data for each individual principal and for the entire group as themes emerged across the database. 
Principals in the study reported that they intentionally gave up their positional power in order to promote shared governance of their schools. They identified a number of strategies used to promote teacher empowerment, including (a) building trust, (b) encouraging teacher expression/voice, (c) hiring staff who embraced collaborative decision making and accepted responsibility for greater levels of involvement in school affairs, (d) encouraging team-building to unify the staff around common goals, (e) providing information for teacher decision-making, (f) supporting teachers in confrontations with central-office administrators, $(\mathrm{g})$ including parents and students in decision-making processes, and (h) encouraging and facilitating action research for problem-solving.

The principals described a number of teacher-related outcomes to shared decision making, including higher levels of classroom and school-wide efficacy, communication, teacher experimentation, morale, and sense of community within the school. Several participants in the study reported their superintendents were generally supportive of their efforts at increasing shared governance of the schools, but they also reported ways in which district policies and decisions hindered teacher empowerment. Bureaucratic processes and the perception that the principal should make all decisions sometimes created obstacles to shared governance. Additionally, the principals noted a number of other barriers to shared governance, including their own personal limitations, time, and teacher perceptions. Blase et al. concluded that principals who seek to promote teacher empowerment in their schools should be willing to share power, engage in a number of relationship-building strategies, and utilize structural changes to facilitate the process. 
Hallinger, Bickman and Davis (1996) confirmed the indirect nature of principal influences on student performance in their study of 87 elementary schools. The authors utilized a conceptual framework which suggested that a variety of antecedent variables, including average student socioeconomic status, parent involvement, principal gender, and level of teaching experience impact principal leadership, which in turn influences the instructional climate and instructional organization of the school, which finally shape student achievement—in the case of this particular study, student reading achievement.

The sample for the Hallinger et al. study included 87 Tennessee elementary schools where the superintendent had agreed to allow school participation in a four-year study of student performance called the School Incentives Improvement Program (SIIP). Principals at participating schools completed an instrument providing context and principal demographic information. The researchers measured parent involvement at each school using a 13-item version of the Connecticut School Effectiveness Questionnaire. Teachers and principals completed 275-item survey instruments in the first and third years of the study.

The instrument assessed teacher and principal perspectives in the following areas: (a) factors associated with school effectiveness, (b) organizational variables hypothesized to be associated with student performance, (c) faculty attitudes toward their ability to improve student performance, (d) the importance of various incentives to school personnel, and (e) selected context variables potentially affecting faculty effectiveness. A variety of context and principal variables, as well as constructs from the survey, described constituted independent variables for the study. The dependent variable was increase in student reading scores on the Basic Skills First Test. 
The researchers conducted a path analysis using the EQS structural modeling program. The analysis began with simple, bivariate measures of relationships between variables to test the idea that principal leadership behaviors have a direct effect on student achievement. Such testing revealed no significant relationship. Next, the researchers tested their hypothesized model using a "constrained paths" approach, which suggests a causal connection that is directly linear and in which each variable must impact the other variables in precisely the way the model suggests. Chi-square analysis indicated that the data did not fit a constrained path model. Finally, the researchers analyzed the data using an open-path model and dropped level of teaching experience from the model because it did not correlate with principal leadership behavior. The researchers discovered the data did conform to this open-path model $\left(X^{2}=27.5, d f=19, p<.05\right.$, and Bentler-Bonett Index $=0.911)$

Hallinger et al. (1996) found that in the sample schools the antecedent variables of parent involvement, student socioeconomic status and principal gender (but not the principal's prior teaching experience) had a significant influence on principal instructional leadership behaviors. Schools with higher levels of parent involvement and higher student socioeconomic status were associated with principals who maintained more instructional focus in their leadership. Confirming earlier studies, Hallinger, et al, also found that female elementary principals in the study were more focused on curriculum and instruction matters than their male counterparts.

Also consistent with prior research, Hallinger et al. (1996) found no direct link between principal behaviors and student achievement, but their path analysis revealed an indirect connection mediated through school climate variables. Specifically, the 
researchers discovered a significant positive relationship between principal behavior and the existence of a clear mission on the part of school staff, which linked directly with higher student achievement. The authors concluded that effective principals do indeed affect student learning, but in an indirect way through their influence on school climate.

Following the Blase et al. (1995) line of inquiry, Blase and Blase (1997) investigated the perspectives of principals with a successful history of utilizing facilitative-democratic leadership in implementing shared governance in Georgia public schools. Facilitators with the League of Professional Schools and University of Georgia education faculty recommended principals for the study. A purposive sample $(n=9)$ of principals was selected for in-depth qualitative study over a seven-month period. The authors utilized a protocol of 12 open-ended interview questions to explore principal perspectives on a variety of issues related to shared governance in their schools. The researchers utilized constant comparative analysis to identify patterns and broad categories of attitudes, behaviors and perspectives among the nine principals.

Overall, Blase and Blase (1997) found principals successful in implementing shared governance had a strong commitment to values of democracy and a belief in the efficacy of teacher leadership to increase school effectiveness. The principals exhibited a number of personal characteristics, including patience, maturity and flexibility, a positive attitude, a friendly demeanor, tolerance of diverse viewpoints, a willingness to share power, and a sense of morality about their professional purpose. The researchers emphasized the need for reflective practice and suggested a number of implications for principals who aspire to utilize shared governance strategies effectively based on this study. In particular, the authors recommended that principals should (a) consider their 
basic assumptions about leadership and democracy in school settings, (b) examine their fundamental beliefs about teaching, especially whether their beliefs are consistent with those of the principals in the study, who shared a common understanding that teachers created knowledge in action through their professional practice, (c) consider each school's context for its readiness for shared governance, and (d) consider their own knowledge and skill relative to developing teacher leadership skills, engage in critical discourse, recognize teachers who are content-area and pedagogical experts, and involve teachers as peers and equals in the decision-making process.

While many studies focused on leadership behaviors at the school level (Blase \& Blase, 1994, 1997; Blase, Blase, Anderson, \& Dungan, 1995; Short, 1994), Spillane and Thompson (1997) studied district-level leadership effects on teachers' capacity to implement instructional reforms. Spillane and Thompson utilized a case study approach to identify ways in which "local capacity" for instructional reform emerged in five school districts and administrator roles that either strengthened or hindered the growth of that capacity.

The sample for this study included five school districts in Michigan identified by public education experts as having a reputation for innovation in mathematics and science instruction. These districts ranged in size from less than 500 to more than 25,000 students, and included three mid-sized urban districts, two suburban and four rural districts. The researchers conducted interviews with central office administrators responsible for instructional issues, principals at all levels, and teacher leaders who played key roles in the implementation of instructional reforms in math and science. 
While Spillane and Thompson (1997) found an uneven depth of implementation of state-mandated instructional changes among districts, they also found that leadership behavior in districts with more substantive reform efforts differed from those with weaker reform efforts in significant ways. The researchers discovered these behaviors paralleled the economic theories of building an enterprise's physical, human and social capital. The physical capital represented the district's willingness to allocate the necessary resources of funding and time for teachers to travel, to learn new instructional techniques, purchase new instructional materials, and to collaborate with one another to reflect on successful ways to implement the reform efforts. The willingness of administrators to learn new instructional techniques themselves and to share them with others, a behavior that showed their dedication and commitment to making reform efforts work, served as an example of the human capital behaviors the researchers found in successful districts. Finally, administrators' actively encouraging teachers to collaborate and learn from one another in implementing new instructional techniques represented social capital. Spillane and Thompson found this to be one of the most important leadership behaviors, in that building social capital within the district encouraged new norms of trust, collaboration and obligation to others:

Trust was crucial because it facilitated conversations about instructional reform. . .. Trust was also essential for genuine collaboration among educators, enabling them to work together to develop a shared understanding of the reforms. Moreover, trust created an environment in which local educators were comfortable discussing their understandings of and reservations about new instructional approaches, conversations that were essential for reconstructive learning. (p. 195)

Blase and Blase (1999) found similar results as Spillane and Thompson (1997) when they examined the impact of instructional leadership on teacher levels of 
motivation, self-esteem, sense of security and feelings of support. Blase and Blase conducted a qualitative investigation exploring principals' everyday instructional leadership characteristics and how these characteristics influenced teacher feelings and behaviors relative to their teaching.

The sample for this study $(n=809)$ included full-time teachers enrolled at graduate courses in three universities throughout the United States. Respondents answered an open-ended questionnaire designed by the researchers called the Inventory of Strategies Used by Principals to Influence Classroom Teaching (ISUPICT). After gathering demographic data, teachers described one behavior their principal routinely engaged in that had a positive impact on their teaching. The researchers coded responses according to guidelines for inductive-exploratory research (Blase \& Blase, citing Glaser, 1992; Strauss \& Corbin, 1990), identifying conceptual categories from the data and comparing them to previously emergent categories and subcategories. A second set of coders checked segments of the researchers' analysis to strengthen the trustworthiness of their interpretations.

Blase and Blase (1999) found two overarching themes of positive instructional leadership, made up of 11 specific behaviors, which the authors called the Reflective Growth Model (RGM) for instructional leadership. The first theme involved a set of behaviors the researchers categorized as "talking with teachers to promote reflection," which included (a) making suggestions, (b) giving feedback, (c) modeling, (d) using inquiry to solicit advice and opinions, and (e) giving praise. The second category of behaviors involved "promoting professional growth," and included (a) emphasizing the study of teaching and learning, (b) supporting collaboration efforts among teachers, (c) 
developing coaching relationships among educators, (d) encouraging and supporting the redesign of programs, (e) applying the principals of adult learning, growth and development to all stages of staff development, and (f) implementing action research to inform instructional decision-making.

The researchers found that these principal behaviors impacted teacher attitudes toward instruction in a number of ways: increasing teacher levels of motivation, selfesteem, efficacy, feelings of support, sense of security, reflection and willingness to engage in innovation, creativity and risk-taking. To the extent that these positive effects create a greater capacity for improved student achievement, Blase and Blase (1999) concluded that the Reflective Growth Model can be a useful way to understand how principals could indirectly have a positive influence on student outcomes.

Some researchers during this period began to conceptualize different forms of leadership to describe the kinds of phenomenon Blase and Blase (1999) and others were describing. One such concept was transformational leadership. Studies indicated that transformational leadership approaches contributed to a greater capacity for individuals and organizations as a whole to implement restructuring initiatives, but Leithwood and Jantzi (1999) explored the effectiveness of transformational leadership approaches on actual student outcomes. Transformational leadership seeks to promote capacity and commitment for professional growth in individuals and groups through focusing on organizational mission and relationships, as opposed to simply the traditional managerial functions of school administration. According to Leithwood and Jantzi (1999), transformational leadership involves six leadership and four management dimensions: (a) building school vision and goals, (b) providing intellectual stimulation, (c) offering 
individualized support, (d) symbolizing professional practices and values, (e) demonstrating high performance expectations, (f) developing structures to foster participation in school decisions, (g) staffing, (h) instructional support, (i) monitoring school activities, and (j) community focus. In their study, Leithwood and Jantzi investigated the role of transformational leadership on the part of both principals and teachers and its impact on levels of student engagement, which they speculated would impact student achievement.

The researchers collected data through two surveys of teachers $(n=1,818)$ and students $(n=6,490)$ in a large Canadian school district representing urban, suburban and rural students at 94 elementary schools. Independent variables for the study included a shared sense of purpose and goals on the part of the school staff, levels of school planning, organizational culture, structure and organization, instructional services and policies and procedures. The dependent variable was level of student engagement. The researchers measured independent variables through a 270 -item instrument called the Organizational Conditions and School Leadership Survey. Students responded to a 61item instrument called the Student Engagement and Family Culture Survey to measure the dependent variable. Leithwood and Jantzi utilized the LISREL 8 program to conduct an analysis of covariance measuring whether data conformed to the proposed model of transformational leadership and student engagement. Factor analysis revealed a single factor outcome for all 10 transformational leadership dimensions. The researchers found that transformational leadership had a strong direct effect on school conditions (with a correlation coefficient of .80 ), which in turn had strong direct effects on classroom conditions (.62). Together, transformational leadership and school conditions explained 
$17 \%$ of the variance in classroom conditions. School conditions (but not classroom conditions) had a significant effect on levels of student engagement, suggesting that the principal's role in shaping school conditions indirectly influences student outcomes.

Blase and Kirby (2000) further explored this line of research by examining the responses of teachers to the Inventory of Strategies Used by Principals to Influence Classroom Teaching (ISUPICT) in which teachers identified their principal as open, effective, and participatory. The authors investigated the specific strategies used by these principals and their effects on teachers.

The researchers administered the ISUPICT questionnaire to approximately 1,200 full-time teachers in three states. Similar to the Blase and Blase (1999) study, in Blase and Kirby (2000) teachers: (a) described a detailed example of a strategy or tactic that the teacher's principal used frequently to influence what the teacher does or thinks in the classroom, (b) described this strategy's effects on the teacher's behavior or thinking, (c) described the teacher's perceptions of the principal's goal in using this strategy, (d) assessed the principal's effectiveness in using this strategy based on a 7-point Likert-type scale, (e) described what feelings the teacher experiences as a result of this principal strategy, and (f) rated their principal in terms of openness and effectiveness using a 7point Likert-type scale. Blase and Kirby focused exclusively on the responses of teachers ( $n=836)$ who judge their principal to be open, effective, and participatory in terms of his or her leadership.

The authors used constant comparative analysis to categorize teacher responses and identify specific strategies used by these open, effective and participatory principals. The researchers found over 1,300 discreet behaviors used by open and effective 
principals, which Blase and Kirby grouped into eight overarching strategies: (a) praise teachers' efforts, (b) convey high expectations for student and teacher performance, (c) actively involve teachers in decision making, (d) provide teachers with autonomy to try creative approaches, (e) support teachers by providing materials, training opportunities, and backing in student discipline matters, (f) nudge teachers to consider alternative solutions to instruction and discipline problems, (g) judiciously evoke the power of authority, and (h) consistently model effective practice.

Blase and Kirby (2000) found that these strategies had many positive effects on teacher thinking, attitudes and behaviors, including higher levels of self-esteem and confidence, job satisfaction and creativity. The authors emphasized that these strategies could not simply by prescribed for effective principal behavior, because contextual factors dictate the proportions and priorities of various strategies and approaches, but are congruent with Schön's $(1983,1991)$ perspective of the principal as reflective practitioner, continuously analyzing the local variables in his or her school to assess how to proceed effectively.

Davis and Wilson (2000) found a more complex relationship between principal behavior and teacher levels of motivation, job satisfaction and stress. In particular, Davis and Wilson sought to understand how principal behaviors meant to enhance teacher empowerment affected their motivation, job satisfaction and stress levels. Participants for their study included teachers $(n=660)$ and principals $(n=44)$ in public elementary schools in eastern Washington. Teachers responded to a survey with 7-point Likert-type items asking their level of agreement or disagreement regarding the extent to which they felt motivation to do their jobs, satisfaction with their jobs, stress on the job, and the 
extent to which their principal engaged in specific empowerment behaviors. Principals likewise responded to portions of the survey regarding their own perceptions of how much they engaged in behaviors designed to empower teachers. The levels of principal empowerment behaviors served as independent variables for the study, and levels of motivation, satisfaction and stress served as dependent variables.

The researchers used the Pearson correlation statistic to identify significant relationships between principal behaviors and teacher reports of motivation, stress and satisfaction, and between principal and teacher reports of principal empowerment behaviors. The researchers found that principal empowerment behaviors significantly correlated to teacher levels of motivation $(r=.38 ; p<.01)$, but not to teacher levels of job satisfaction or stress. In comparing the variables of motivation, satisfaction and stress with one another, Davis and Wilson (2000) found that motivation significantly correlated to both job satisfaction $(r=.56 ; p<.01)$ and job stress $(r=-.53 ; p<.01)$. In other words, by positively affecting teacher levels of motivation, principals indirectly exerted a positive influence on teacher job satisfaction and stress levels.

Keedy and Simpson (2001) argued that norms of behavior indicate a school's capacity for reform better than simple structural changes or enhancements, and that as norms of behavior moved toward relationships of trust, openness and collaboration, capacity for real reform increased. Whereas Spillane and Thompson (1997), Blase and Blase (1999), and Davis and Wilson (2000) focused on principal-teacher relationships by looking at specific influences of principals on the affective dimension of teachers' work lives, Keedy and Simpson (2001) explored ways in which principals and teachers exerted reciprocal influences in terms of the more general school-wide norms of behavior. 
Taking a socio-cultural approach to understanding organizational dynamics, Keedy and Simpson (2001) investigated how influence flowed among staff in four U.S. high schools with state-wide reputations for strong, positive leadership. The study involved a purposive sample of four high school principals in a southeastern state identified by education agencies and professors of school leadership for having a reputation for "turning schools around" and improving student achievement during their tenures. The researchers conducted week-long observations at each school and interviewed principals for approximately one hour each day about the school's contextual conditions when the principal tool over leadership of the school, the principal's biographical influences and values, vision and specific behaviors taken based on these values and influences.

The researchers also individually interviewed a representative sample of ten teachers from each school, including some department chairs, about ways their principal contributed to school improvement, how they interpreted their principal's behavior, and what norms defined the 'way things work' in terms of their relationship with their principal. The researchers conducted focus group interviews with four to six teachers per school at the end of the visit to clarify the emerging norms of behavior for each school. From the focus group responses, the researchers generated a checklist of school-based norms and distributed the list to all teachers in the school, asking them to mark their perceptions of each norm ("yes" or "no" meaning they believed the norm was operable or not operable for their school, or "unsure"). The checklist instrument achieved a 73\% response rate with a Cronbach alpha of .83 . 
Keedy and Simpson (2001) analyzed transcripts of teacher interviews to inductively identify common characteristics, collapsing categories until four education administration professors reached an inter-rater agreement level of .80. Likewise, the researchers analyzed transcripts from principal interviews for common patterns of principal behavior, and then checked these interpretations with categories that emerged from teacher interviews. Next, the researchers identified the percentage of agreement among teachers and between teachers and principals as to the presence of specific norms of behavior in their school. Finally, the researchers used the collected data to discover flows of influence between teachers and principals and the degree of reciprocity in this flow.

From thick descriptions and data analysis for all four schools, Keedy and Simpson (2001) found that principals exerted a strong influence over norms of behavior within their schools (teachers confirmed ten of the eleven principal priorities as norms in their schools). In two of the four schools, teachers also exerted a reciprocal influence on the principal. In the third school the principal seemed to take a much more "corporate" approach toward management of the school and did not invite or welcome teacher influence in his decision-making. In the fourth school, the principal had a nearly singleminded focus in priority that was somewhat impenetrable by teacher influence. Overall, Keedy and Simpson (2001) found more principals had great influence over the norms of their schools even when the teachers did not necessarily endorse or condone those norms.

Keedy and Simpson's (2001) study was important, in part, because it provided richly descriptive information about the role of principals in high schools, which have been understudied relative to elementary schools in the research on principal effects. 
Leithwood, Steinbach, and Jantzi (2002) furthered the exploration of principal behaviors at the secondary level, and investigated how principal leadership could influence teacher motivation to implement accountability policies.

Leithwood et al. (2002) conducted a study in the Canadian province of Ontario five years after the initiative of a major, province-wide reform effort designed to restructure school governance and finance and increase school accountability for student performance. The researchers sought to confirm an understanding that teacher motivation toward implementation of accountability measures was shaped by their perception of policy outcomes, their perceptions of the goals of reform, their beliefs about their personal capacity to implement changes, their perceptions about contextual factors which would support or hinder reform, and emotional arousal processes.

The sample for this study included teachers $(n=48)$ and school administrators $(n$ $=15$ ) in five secondary schools in south-central Ontario located in four different districts, ranging in size from 80,000 to 300,000 students. The researchers randomly selected teachers within schools and administrators included the principal and assistant principals from each school. Because the researchers sought to explore the complex relationships between a number of personal and context-specific variables, they chose a qualitative research design. The researchers interviewed participants using a semi-structured questionnaire and asked participants to identify government policies aimed at increasing school accountability and what effects these policies had made on schools. The researchers then coded transcripts of the interviews using the theoretical framework as a guide. 
Results indicated that teachers and principals identified 15 specific accountability policies. Sixty-seven percent of teachers reported negative responses to the accountability measures, with only $14 \%$ expressing positive attitudes toward the changes. Most of the negative attitudes had to do with the difficulty of implementing the reforms. Slightly more administrators viewed the policies in a more positive light. Fifty-one percent of administrators had negative feelings toward the policies, and $22 \%$ had positive feelings. The majority of teachers doubted the actual goal of reforms involved improving student achievement, believing instead that political goals motivated the policies. Again, administrators were slightly less skeptical than teachers were about the goals of reform, with more than half (53\%) identifying improved student outcomes as the goal.

In terms of their capacity to implement change, teachers and administrators both agreed that the new accountability measures had eroded their sense of professional efficacy, and doubted the adequacy of time, resources and training to implement reforms successfully. Both groups also doubted whether the sincerity of support from policymakers to make the reforms effective. In terms of emotional response to these changes, teachers and administrators reported feeling higher levels of anxiety, frustration and resentment.

In general, Leithwood et al. (2002) found teachers and administrators much more motivated to implement reforms unambiguously intended to improve student achievement, but had strongly negative feelings toward the overall goals for reform, the lack of resources and unrealistic timelines for implementation, and the non-consultative processes used to formulate new policies. The researchers found that teachers accepted their accountability to student and parents for student achievement, but did not feel 
accountable to policymakers. The researchers conclude that this is the key component for sincere implementation of school reforms, but policymakers should focus more on commitment strategies (i.e., tapping into teachers' intrinsic commitments to help students) rather than using control strategies. Leithwood el al. believed this was most effectively done at the local school level, a conclusion confirmed by Sirotnik (1989) and by Keedy and Achilles (1997).

Anderson (2004) agreed with the conclusions of Leithwood et al. (2002) that teacher attitudes and leadership were keys to successful change efforts at the local level, and echoed themes explored by Keedy and Simpson (2001) regarding the reciprocal influences of teachers and principals. Anderson (2004) conducted a qualitative case study investigation of six Canadian schools identified as having a reputation for high levels of teacher leadership and examined flows of influence among teachers and between teachers and principals.

The study's sample included teacher leaders, teachers who had identified others as teacher leaders, and principals $(n=28)$. Concerned that previous research on teacher leadership was biased because of the presence of large numbers of teachers with formal positions of authority within the school (such as department heads), the researcher specified that these were teachers whose leadership was manifest in an informal role. The researcher interviewed participants about teacher leadership roles and influences, and coded responses according to general categories of meaning and frequency of occurrence.

Anderson (2004) found that teacher leaders exerted a clear influence on principals, and both teacher leaders and the principals themselves recognized this influence. In particular, principals relied on the expertise, experience and insight of 
teacher leaders in shaping school policy and making decisions. Likewise, Anderson found that principals exerted an influence on teacher leaders through a complex relationship tied to teacher leaders' openness to this influence and the ways in which principals nurtured trust with teacher leaders. In other words, Anderson confirmed a reciprocal flow of influence between teacher leaders and principals and offered three models to categorize the different forms these interactions take: (a) a buffered model, in which the principal is close to a key group of teacher leaders, but is relatively isolated from other teachers in the school, (b) an interactive model in which the principal has influence over a wide variety of the school's teachers, including key teacher leaders, and (c) a contested model, in which the principal is in conflict with teacher leaders and compete with them for influence over the rest of the staff. Anderson (2004) concluded that the interactive model presented the greatest capacity for effective decision-making and sharing of leadership roles, and furthered research in principal effects by confirming the positive role principals could play in creating more empowering work environments for teachers.

Barnett and McCormick (2004) investigated the impact of transformational leadership on school culture, citing previous literature that such a connection could indirectly promote higher student achievement. The researchers examined secondary schools in Australia and explored what dimensions of transformational, transactional and laissez-faire leadership were practiced in the schools, what dimensions of school culture were present in the schools, and the relationships between the leadership approaches and school learning culture. 
Participants for the study included 373 randomly-selected teachers from 41 schools that agreed to participate. The teachers responded to the Multifactor Leadership Questionnaire, which included 45 Likert-type items that measured the degree to which teachers believed their administrators exhibited transformational, transactional or laissezfaire leadership behaviors. Participants also responded to the Patterns of Adaptive Learning Survey, a 42-item Likert-type survey that measured the degree to which teachers agreed that certain dimensions of school culture were present in their schools. The researchers used a combination of multilevel analysis and structural equation modeling to analyze the data to address both problems with observations when data are clustered into groups and to allow for comparison of relationships across levels and finally to account for differences among both schools and individual teachers.

Confirmatory factor analysis revealed two dimensions of transactional leadership, vision and concern for the individual. In contrast, transactional leadership was identified by passive behaviors. These results were not entirely consistent with the author's proposed models for distinct forms of transformational, transactional and laissez-faire leadership. The authors concluded that the teachers in the study did not distinguish between the various dimensions of the model in their perceptions and understanding of their principals' leadership. Factor analysis also revealed dimensions of school leadership in the schools, but the data did not perfectly confirm the model the authors proposed. The data did fit the overall model, however, suggesting that statistically significant differences in leadership explained variation in the school cultures. The model was able to eliminate variables such as principal or teacher gender and tenure, principal age, and school size in explaining the differences in school culture. 
The structural equation model found a significant effect of a principal's concern for individuals (associated with transformational leadership) on the principal's capability to articulate a clear vision, which in turn had a positive effect on teacher levels of taskfocus and appreciation for excellence in teaching. Likewise, Barnett and McCormick (2004) found that passive leadership had a negative effect on teacher task focus. The authors concluded that data confirmed the importance of at least two dimensions of transformational leadership and that these dimensions had a significant positive role in shaping elements of school culture.

Basom and Frase (2004) reviewed research over many years that further confirmed the idea of the indirect influence of principals over school culture, and suggested that principal behaviors could directly impact the work environment of schools in a positive way for teachers. Basom and Frase (2004) framed their study in terms of concepts developed by Csikszentmihalyi (1990), whose work in psychology explored the experience of "flow," a "state in which people are so involved in an activity that nothing else seems to matter; the experience itself is so enjoyable that people will do it even at great cost, for the sheer sake of doing it" (cited in Basom \& Frase, 2004, p. 241). According to the authors, many people mistakenly associate the experience of "flow" solely with physical experiences such as athletic activities, but Csikszentmihalyi (1990) found the experience present in many different work environments and was key to intrinsic motivation: opportunities for flow greatly enhance an individual's motivation to engage in the activity. Basom and Frase applied the concept to teachers' classroom experiences, and reviewed qualitative and quantitative research studies, finding that many teachers regularly reported "flow experiences" during classroom teaching. 
Basom and Frase (2004) found that the following conditions contributed to teacher flow experiences: (a) frequent principal visits to the classroom and principal attention to issues of curriculum and instruction, accompanied by principal encouragement of teacher reflection and growth, (b) effective professional development that meets teachers' individual needs and encourages innovation and experimentation, (c) teacher evaluation in the context of frequent principal visits, (d) strong levels of teacher self-efficacy and teacher-perceived efficacy of other teachers, and (e) high levels of student cognitive engagement. Based on these findings, Basom and Frase offered a list of recommendations to school leaders for increasing teacher flow experiences, including (a) making frequent, meaningful classroom visits, (b) protecting instructional time and minimizing classroom disruptions, (c) promoting quality professional development, and (d) giving teachers time to collaborate, reflect and organize with one another for more effective classroom instruction:

Building school environments in which teachers and other workers have frequent opportunities to experience flow requires a new view of leadership and "followership." School improvement will depend upon principals who can foster such conditions - conditions necessary for sustained education reform in a complex, rapidly-changing society. (p. 254)

Basom and Frase's (2004) study supported Keedy and Achilles's (1997) argument that improving work conditions and relationships within schools is the key to making substantive education reform successful.

Finally, Timperley (2005) found that in distributed leadership environments, key teachers could play as important a role in shaping the learning environment of the school as principals could. Previous literature indicated the traditional conception of the "heroic" solo principal as the key player in improving school effectiveness was no longer 
helpful. The ranks of potential and practicing school administrators lack such heroic leaders in sufficient numbers to make this a realistic vision, and most principals find the number of mundane responsibilities in their jobs preclude such heroic activities in the first place (Timperley, citing Copland, 2003; Elmore, 2002; Gronn \& Rawlings-Sanaei, 2003). Of greater interest to Timperley was the concept of distributed leadership, in which leadership is conceived as activities and interactions for school improvement that are widely distributed across personnel and situations and involve network patterns of control. Specifically, Timperley sought to understand how different forms of distributed leadership were differentially effective.

Timperley studied seven New Zealand schools participating in a literacy improvement program. These schools were located in low socio-economic areas of the country with traditionally low student performance. Each school had a teacher literacy leader responsible for facilitating team meetings of first grade teachers. The researcher conducted observations of team meetings and interviews with the literacy leader, principal and three teacher team members from each school over a three-year period. She also analyzed student achievement data from each school during a four-year period. Timperley analyzed transcripts of group meetings and coded the primary topics of discussion. She found topics of discussion varied between two groups of schools. Group one schools either had no student achievement data available or their data was aggregated at the whole-school level and so discussions about particular students or individual teacher activities was not possible. Teacher team meetings at these schools focused mostly on program issues and teaching approaches, but not teaching implications. Group two schools, on the other hand, had specific student achievement information available 
and their discussions focused primarily on teaching implications arising from the student achievement data. (It should be noted that in New Zealand at that time there were no nationally or locally-mandated student testing or school accountability systems).

Timperley examined average student reading scores at the end of the first grade and averaged these scores for a four-year period (using the first year as a baseline), transforming the data into $z$-scores for cross-school comparisons. Utilizing one-way analysis of variance (ANOVA) and the Sheffé test of multiple comparisons, the researcher found that student scores in Group Two schools (where meetings focused on teaching implications of student achievement data) were significantly higher than Group One in the first two years of the study. In year three, the meeting activities of all the schools became more similar (because nationally-normed student achievement data became available), and there were correspondingly significant increases in student achievement at the Group One schools.

The researcher found that Group Two schools had a more clearly-focused embedded vision in their team meetings that centered around using data to identify specific students who needed additional or differentiated instruction, collaboratively developing interventions for those students, and assessing progress. Group One schools lacked this embedded focus and their discussions centered almost entirely around implementation of the instructional program rather than on individual student performance. Analysis of meeting transcripts revealed that teachers in Group One schools spent more time focused on external factors that influenced student achievement than on their individual responsibility for student success. Likewise, teacher literacy 
leaders who facilitated team meetings were more focused on improving individual student performance in Group Two schools.

Timperley also found Group Two schools exhibited a broader sharing of responsibility for instructional improvements among principals, literacy leaders and teachers. The literacy leader effectively functioned as a "boundary spanner" in intentionally encouraging this sharing of responsibility (Timperly, 2005, p. 409). Principals played an important role by fostering a spirit of cooperation while promoting a vision that program success was dependent upon improved student achievement. In Group One schools, principals tended to believe that program success was the equivalent to program implementation. Literacy leaders in the effective schools were able to facilitate coherence between the principal's beliefs and activities and those of the teachers.

Timperley's study (2005), while not specifically focused on principal leadership, indicated that principals played an important role in school improvement through their beliefs and assumptions about student achievement and their willingness to share power with teachers. Timperley concluded that an effective distributed leadership approach held promise for enhancing student performance.

In summary, literature on school reform suggests that for reform efforts to succeed, schools must ultimately be transformed into centers of inquiry (Sirotnik, 1989). That schools remain the targets of change rather than centers of inquiry is not surprising given the orientation toward control, isolation and autonomy described by Cusick (1992) and the historical schism between theory and practice described by Keedy (2005). Research shows that principals can in fact have a positive impact on school culture by 
encouraging teacher empowerment through self-managed teacher leadership teams (Davis \& Wilson, 2000; Short, 1994), encouraging teacher networking and building relationships of trust (Spillane \& Thompson, 1997), engaging in specific instructional leadership practices that increase teachers' motivation and self-efficacy (Basom \& Frase, 2004; Blase \& Blase, 1999), and sharing leadership and being open to reciprocal influences from effective teacher leaders (Anderson, 2004; Keedy \& Simpson, 2001). These positive effects are examples of changes in normative thinking and the quality of relationships that Keedy and Achilles (1997) suggested had the greatest leverage for meaningful school change.

Keedy and Achilles (1997) proposed the development of teacher- and principaloriented theories of practice as a central tool for changing the normative thinking of schools and thereby bridging the gap between theory/research and practice. Theories of practice are techniques in which "practitioners actively and persistently analyse their assumptions in relation to the grounds supporting their practice" by critiquing the assumptions that lie beneath their decisions and actions, articulating alternate assumptions, values or beliefs, and testing the effectiveness of these new assumptions in the context of their own work environments, i.e. their schools and classrooms" (Keedy \& Achilles, 1997, p. 5). The results of testing these new "theories of action" inform a continuous loop of inquiry, testing, learning, and inquiry. Argyris and Schön (1974) first operationalized this approach which utilizes a system of "double-loop learning" that has the potential to expose and transform normative thinking in the way Keedy and Achilles suggested. Keedy (2005) pointed to the work of Argyris and Schön (1974; 1978) as an example of how theories in practice could be operationalized into a system that 
individuals and groups can intentionally use to understand how theory can be transformed into experimental action, which can then in turn influence the development of theory in an ongoing process of learning and discovery.

Theories of Practice

Argyris and Schön (1974) first articulated the concept of theories of practice, which involves engaging professionals in a process of self-reflection to discover the values, beliefs and assumptions that guide their decision-making (their theories of action), articulate new strategies for problem solving, perhaps based on new values, beliefs and assumptions and experiment with these new theories of action for enhanced professional effectiveness (called double-loop learning). This section provides an overview of empirical studies that have attempted to measure the use and effect of theories of practice in a variety of settings.

Lipshitz (2000) assessed the long-lasting influence Argyris and Schön made to organizational theory, and expressed admiration for the complexity, rigor and thoughtfulness of Argyris and Schon's model for organizational learning. Lipshitz argued that research has done little to empirically explore Argyris and Schon's concepts for a wide variety of reasons. Lipshitz argued that key components of their model deserve greater attention from researchers. Some empirical studies, however, have attempted to explore how Argyris and Schön's concepts work in practice.

In his own work, Schön $(1983 ; 1987)$ argued that the concept of theories of practice had considerable implications for the work of professional practitioners. Schön advocated what he called "reflective practice," by which practitioners would engage in "reflection-in-action," seeking to uncover their espoused theories and consciously 
experiment and test new theories of action, possibly based on revised values, beliefs and assumptions. According to Schön, the ability to engage in reflection in action was what distinguished expert practitioners from novices. Kirby and Teddlie (1989) sought to fill a gap in quantitative literature on reflective practice by developing an objective instrument to assess a teacher's perceived engagement in reflection-in-action.

Kirby and Teddlie (1989) based their work on the specific features of Argyris and Schön's (1974) model, by which a reflective practitioner would engage in (a) diagnosing the source of problems, (b) test theories and make decisions based on observable data, and (c) take personal responsibility for outcomes. Kirby and Teddlie (1989) based their Reflective Teaching Instrument (RTI) on these three dimensions. The researchers initially developed 60 Likert-type items, with 20 items representing each sub-dimension, and presented the draft instrument to a panel of experts in educational administration to develop content validity, eliminating items with less than $75 \%$ agreement, and reduced the instrument to 48 items. The researchers then pilot-tested the instrument with 47 teachers for validity and reliability and further reduced the instrument to 26 items, then field tested the final instrument with a sample of teachers $(n=102)$ from a local public school district.

Kirby and Teddlie (1989) found the reliability of the diagnosis sub-scale to be slightly less than acceptable (.65). Factor analysis for construct validity did reveal three factors, which yielded a 15 -item scale that represented the sub-domains of testing diagnosis and personal causation and accounted for $44.6 \%$ of the variance in responses. The researchers recommended further testing with the instrument, and called for empirical studies to explore these dimensions of reflective practice. 
Kirby and Paradise (1992) further tested the Reflective Teaching Instrument (RTI) in a study which investigated whether teacher scores on the RTI predicted teacher effectiveness. The sample included elementary teachers $(n=52)$ from a large suburban public school district. The independent variable was teacher scores on the three subdomains on the RTI; the dependent variable was teacher scores on the Virgilio Teacher Behavior Instrument (Teddlie, Virgilio, \& Oescher, cited in Kirby and Paradise, 1992). The researchers conducted a multiple regression analysis to estimate the relative contribution of each of the three factors of reflective teaching in predicting teacher effectiveness and found that the personal causation factor was the only significant predictor of teaching effectiveness. Kirby and Paradise (1992) called for further empirical studies using the RTI.

Meanwhile, researchers in the field of organizational behavior explored other aspects of Argyris and Schön's $(1974 ; 1978)$ model and attempted to replicate learning techniques that Argyris and Schön had used with students in their consulting seminars to assist professionals in moving from Model I to Model II behaviors. Using students in their own seminars and courses in management and organizational behavior, Friedman and Lipshitz (1992) conducted a case study and attempted to mimic an intervention Argyris and Schön had developed called the "X-Y exercise."

In the $\mathrm{X}-\mathrm{Y}$ Exercise, students analyzed a case in which manager $\mathrm{X}$ confronted a subordinate $\mathrm{Y}$ using Model I behaviors. The goal of the X-Y Exercise, however, is to see whether the students will exhibit Model I behaviors themselves in their critique of manager X's behavior, and whether the students can then modify their reactive behavior (a process Argyris called "unfreezing"). Argyris (1982) reported that invariably, students 
did exactly what manager $\mathrm{X}$ did in the case, but typically did not realize this until confronted by the teacher. Argyris found that students frequently became defensive during this confrontation, but those more open to self-reflection gained a much deeper understanding of Model I and Model II concepts. Argyris termed students who were so unconscious of their own theories of action "low learners," and advocated using the process of confrontation to raise the students' awareness.

Friedman and Lipshitz (1992) found similar patterns of behavior in their own students when confronted with the X-Y Exercise, but found that confronting students with their Model I behavior typically led students to withdraw and distance themselves from the learning process, rather than to engage it more deeply. Friedman and Lipshitz experimented with modified approaches to the X-Y Exercise and reported the results in their case study.

Friedman and Lipshitz (1992) discovered that Argyris (1982) spent far more time than they typically did teaching students the core concepts of Model I and Model II behavior, usually after the X-Y Exercise. In their case study, the researchers experimented with offering a much lengthier introduction to Model I and II concepts prior to the X-Y Exercise. During the exercise itself the students as usual reacted with Model I behavior, but the researchers engaged in less confrontation with students, simply allowing them to withdraw if they chose without confronting the behavior. The students then wrote reflective papers on the X-Y Exercise and applied their insights to personal case studies of their own. Friedman and Lipshitz found that students had a much stronger grasp of Model II concepts and were more self-conscious about ways in which they 
themselves had exhibited Model I behavior during the X-Y Exercise than previous cohorts of students were.

Friedman and Lipshitz (1992) concluded that confronting student resistance was less important than Argyris originally assumed. They recommended that the X-Y Exercise could be helpful for teaching professionals the process of "unfreezing" their old behaviors and assumptions, and that student withdrawal from the process did not necessarily mean they were not processing the lessons of the exercise. Friedman and Lipshitz suggested that instructors give students plenty of time to learn and absorb Model I and II concepts, and that instructors not necessarily conclude from a student's defensiveness or withdrawal that they were "low learners."

While Friedman and Lipshitz (1992) focused on how to teach reflective action to professionals, Ferry and Ross-Gordon (1998) questioned whether experienced educators were necessarily more skilled at reflective practice than novices (an implicit assumption in Schön, 1983, 1987), but found validation of many other aspects of Schön's framework.

Ferry and Ross-Gordon (1998) conducted a qualitative investigation of family living educators $(n=52)$ who worked for state extension agencies to determine if a difference existed between novice and experienced educators in terms of their use of reflection-in-action. The researchers administered a questionnaire featuring a problematic professional situation and asked participants to respond in writing, describing how they would address the problem. The researchers followed up the questionnaires with a randomly selected group of novice $(n=8)$ and experienced $(n=10)$ educators during which the researchers presented three more problematic situations and asked the participants to respond orally in a "think-aloud" format. 
The authors classified educator responses based on the presence of six indicators of Schön's reflection-in-action process: (a) recognition of the problem; (b) recognition of incongruities; (c) evidence of reframing of the problem; (d) generation of new solutions; (e) testing-in-action of solutions; and (f) evaluation of outcomes. Though the authors acknowledged that "reflection" is not a dichotomous variable, for purposes of the study, Ferry and Ross-Gordon decided to designate an educator as "reflecting" if her response exhibited four of the six indicators, and non-reflecting if her response included one or none of the indicators.

The researchers found more differences between reflecting and non-reflecting educators than between novice and expert educators. In particular, reflecting educators (whether novice or experienced) took a much more interactive and data-gathering approach when framing a problem, whereas non-reflective educators tended toward a more instrumental problem-solution approach, applying their technical skills to address problems (usually deemed as a situation when "things didn’t go as you planned”) to resolve the situation as quickly as possible. The reflective educators looked outside of the parameters of the problem itself to understand the situation and articulate new ways of understanding it. Likewise, non-reflecting educators approached solving problems in a process that involved applying a solution, and if the solution did not work, trying another solution, whereas reflecting educators played out various scenarios and questioned whether inconsistencies or misperceptions in their reading of the situation influenced their original or understanding of the problem. Finally, reflective educators engaged in a process of looking back at their decision-making process, what Ferry and Ross-Gordon called "a reconstructive mental review" to identify misconceptions in their own thinking 
and ways to enhance their effectiveness in the future. Non-reflective educators, on the other hand, reported little or no use of reflection-in-action.

Ferry and Ross-Gordon (1998) concluded that an educator's approach to problem solving was a much better gauge of whether she was a reflective practitioner than level of job experience. The authors found that their data confirmed many of the indicators Schön $(1983,1987)$ used to describe reflection-in-action, but called for further study on why some professionals have internalized reflective practice and others have not.

Tsangaridou and O'Sullivan (2003) extended research on the reflective practice of educators in a study investigating whether physical education teachers exhibited the incongruity between their espoused theories and theories-of-action, as Argyris and Schön (1974) suggested. The purposive sample for their study included four experienced elementary and secondary physical education teachers in the U.S. The researchers conducted non-participant observations of the teachers in their classrooms to assess the teacher instructional practices, which equated to their theories of action and videotaped classes. The researchers asked participants to keep a written or oral journal for the observed class periods to provide data on teacher reflective processes related to their teaching. The researchers conducted informal interviews with the teachers before and after each observation, and three formal interviews about teacher philosophies of teaching (equated with "espoused theories").

Tsangaridou and O'Sullivan (2003) reviewed transcripts from the interviews, field notes, videotapes and teacher reflective journals using an inductive, cross-case analysis approach. Three major thematic clusters emerged: curricular, pedagogical, and social, which described both teacher espoused theories of instruction and their behaviors in the 
classroom. To increase trustworthiness of their interpretations, the researchers engaged in peer debriefing where the study participants reviewed transcripts and offered corrections of inaccurate information or interpretations.

The researchers found that the teachers uniformly shared a similar espoused theory of instruction, and consistency between their pedagogical practices and their espoused theories. The researchers did not conclude, however, that these teachers necessarily modeled reflective practice. The authors noted for example that the teachers shared an overriding focus on skill development on the part of their students (as opposed to a more general awareness of physical health issues), and that this focus reflected both their beliefs and their practices. Tsangaridou and O'Sullivan's (2003) study therefore offers an important examination of the congruence between espoused theories and theories in action, but indicates that mere congruence does not automatically imply reflection-in-action.

In a more recent research study designed to investigate how theories of action explain interpersonal dynamics in multi-disciplinary professional teams, Rogers (2004) conducted a qualitative inquiry of an interprofessional medical team in Australia. Specifically, Rogers sought ways to apply theories of action to solve a professional dispute between a manager and a subordinate. Rogers studied the behaviors of Allison, the manager of a small interprofessional community health care organization in Australia. The author did not describe his specific research methods, but wrote that he engaged in in-depth investigation based on the action science approach to problems of situated practice (Argyris, Putnam, \& Smith, 1985). The manager in Rogers's case attempted to deal with a subordinate, Ms. X, who was responsible for dealing with an external 
organization that functioned as a sub-team of the main interdisciplinary work group under study. According to the case notes, members of the subgroup felt that Ms. X was taking control of the group, limiting member autonomy, and being excessively directive of other professionals' work. To address the problem, Allison discussed the matter with Ms. X and encouraged Ms. X to lessen her involvement with the subgroup, framing her concerns as a matter of time constraints and excessive workload. To Allison's surprise, Ms. X reacted with hostility and negativity, accusing Allison of being excessively directive and violating Ms. X's professional autonomy to conduct her work as she saw fit.

The researcher examined the case from a theory of action perspective, and concluded that by being indirect, unilateral and manipulative in her approach, Allison engaged in what Argyris and Schön called "Model I" behaviors, which invariably leads the subordinate to greater levels of resistance, isolation and defensiveness. Moreover, Rogers discerned a possible gap between Allison's espoused theories regarding staff autonomy and her theories in use, a discrepancy that Argyris and Schön believed was common. Rogers believed that members within the organization itself had widely divergent views about autonomy, and cited Argyris and Schön in recommending open discussion among team members as to their varying beliefs in an effort to articulate a common vision for how this value ought to be realized in their workplace. Finally, the author believed that differences in the professional practices of different disciplines might explain some of the variance in viewpoints regarding theories of action in interdisciplinary teams such as Allison's.

Rogers (2004) concluded that Allison's case study illustrated the applicability of Argyris and Schön's Model I and Model II concepts. In recommending next steps for 
Allison based on theory of action principles, Rogers recommended that Allison engage in self-inquiry as to the values, beliefs and assumptions that motivate her professional practices regarding staff autonomy and to deliberately utilize a Model II approach to engage her staff in an open discussion about their viewpoints regarding this issue.

Finally, Rogers recommended that the staff collectively engage in testing new theories of action regarding staff roles and autonomy in the vein of Schön's reflection in action:

"Such research is necessary to test the hypothesized effects of model II values to facilitate a self-correcting system that can promote learning even under pressure to revert to model I" (Rogers, 2004, p. 247).

Despite the research into teacher and other professionals' use of reflective practice, few studies have been conducted on the use of theories of practice by school principals. Erlandson (1994) explored the professional needs of school principals and found that school leaders had a need for ongoing reflective practice throughout their careers, confirming Shön's $(1983 ; 1987)$ advocacy for reflection-in-practice on the part of professionals.

Erlandson (1994) conducted a mixed-method, exploratory study of principal professional development needs throughout their careers. The sample for the study included two focus groups of school principals: one group of relatively inexperienced principals $(n=10)$ and another of more experienced principals $(n=9)$. Other participants included principals $(n=97)$ who responded to an open-ended questionnaire on their professional development priorities, and principals from Texas $(n=5)$ and London, England $(n=6)$ who agreed to let the researcher review their own professional development plans. The study also included interviews with a purposive sample of five 
additional principals. The researcher analyzed transcripts from focus groups and interviews and content analysis from the review of professional growth plans and openended surveys to identify categories of professional development needs.

Erlandson (1994) found that while early in their careers principals placed a high priority on learning basic technical skills and more experienced principals put higher value on interpersonal and social skills, throughout their careers principals required ample opportunities for reflective practice.

Polite (2000) offered a case study of one district's efforts to help its principals increase their reflective practice. Polite reported on a two-year program conducted between 1997 and 1999 in the Jefferson County (Kentucky) Public Schools (JCPS) intended to broaden principal approaches to problem solving to involve long-term reflection in action:

Reflective practice challenges urban principals to no longer think in terms of how (technical) to solve problems, but why (critical) when considering a particular solution, and what (interpretive) message that decision(s) sends to the school community. (Polite, 2000, p. 4)

The district invited a random, stratified sample of JCPS principals $(n=19)$ to participate in the two-phase program. In the first phase, a specially-trained professional development coach observed each principal for two full days, during which time the coach gathered observational data on the principal's work habits and established rapport. In the second phase of the program, each principal wrote a reflective plan to address some specific need of his or her school or some aspect of his or her professional practice. The principals structured the reflective plan based, in part, on Schön's (1983) concept of reflective practice. The researcher reported that in most cases the target for the reflective plan emerged from feedback offered by the coach. The plan involved a multi-step 
process: (a) "Visioning - Critical Reflection," which meant articulating the problem and the desired outcome, (b) "Reflection for Action," or considering all the current contextual factors that shaped the situation, (c) "Technical Reflection," or outlining the specific steps in addressing the problem, and finally (d) "Interpretive Reflection," which occurred after the implementation to reflect on the process itself and the principal's performance.

Polite (2000) reported on four case studies of individual principals and their experiences with the individual reflection plan. The author found that all the principals reported feelings of enhanced effectiveness and believed that the reflective process helped them understand problems more clearly and gain new insights into their professional practice. Principals also reported a lack of training in their professional preparation for reflective thinking processes, and expressed frustration over the fragmented nature of their work time and limitations it placed on their ability to engage in reflective practice.

Day (2000) explored the professional behaviors of principals in the United Kingdom identified by government education inspectors as "good" or "excellent." The researcher found that these principals consistently exhibited behaviors that could be described as reflective practices.

The researcher studied principals $(n=12)$ in the United Kingdom who had reputations for being successful leaders who were able to increase student achievement. The author conducted over 200 interviews with parents, teachers, students, governors (board members) and the principals themselves and analyzed responses for emerging patterns of principal behavior. The author did not describe the specific methods used to analyze data collected in the study. 
Day discovered that principals in the study engaged in various kinds of professional reflection, including reflection on the vision and culture of the school, on pedagogical practice, on interpersonal relationships with staff, students and parents, and intrapersonal reflection on their own personal motivations and professional needs. Day's study confirmed Schön's (1983) understanding that technical knowledge has significant limitations in terms of practical professional problem solving, and that a more holistic approach to professional reflection is needed:

Reflection involved principals in these schools in a critique of practice, the values which were implicit in that practice, the personal, social, institutional and broad policy contexts in which practice takes place, and the implications for these for improvement of that practice. (p. 123)

Day concluded that learning these multi-faceted approaches to reflective practice was critical for enhancing principal professional effectiveness.

While empirical studies have focused on the various elements of Argyris and Schön's model in terms of professionals other than school principals, or on the broader topic of "reflective practice," far fewer studies have explored ways to measure the cognitive processes that make up theories of practice. Allison and Allison (1993), Leithwood, Steinbach, and Raun (1993), and Ruff and Shoho (2005) conducted studies that advanced research into principal cognitive processes for problem-solving and may suggest directions for future study regarding principal use of theories of practice.

Allison and Allison (1993) applied schema theory to investigate how school administrators of varying experience levels approached practical problem solving. The researchers explored connections between experience and the ability to provide both attention to detail and to see the problem on complex, abstract levels. The authors referred to this capacity through the metaphor of looking through both ends of a 
telescope, and suggested that administrators with this ability used more complex schema for problem-solving, and thus exhibited more "expertise" as professionals.

Participants for the study $(n=40)$ included teachers and principals at varying levels of administrative experience, including veterans (with more than 20 years experience), seasoned principals (10 to 15 years), rookies (in their first or second year of experience), aspirant administrators (all of whom were teachers), and entrants to the field of education who were earning bachelors degrees to become teachers. The researchers utilized a think-aloud case study approach to measure participant levels of attention to details within the case and levels of abstract thinking. The participants read aloud from a case study involving a problem with the school library. Researchers then asked the participants to discuss out loud how they would address this problem as the school's principal, and then to describe the cognitive processes they used in articulating the response.

A group of graduate students and educational administration professors then read and rated transcripts of all the responses, assigning a score between 1 and 10 for the overall quality of response (with 10 representing an excellent response). Researchers then used the mean judged expertise rating to divide the participants into three groups: low expertise (bottom quartile), medium expertise (inter-quartile range), and high expertise (upper quartile). Next, Allison and Allison utilized a 3-point coding scheme to assess participant attention to the details of the case, awarding points based on whether each of the 25 emergent dimensions of the case was mentioned (one point), considered (two points), or actively addressed by the subject (3 points), for a total possible score of 75 , reflecting the subject's density of attention. Again, the participants were divided into 
three groups representing low, medium and high levels of density attention. Finally, the researchers coded transcripts for goal abstraction, reflecting on the extent to which the subject approached the problem with concrete or abstract goals. Four increasingly abstract goal categories emerged, including physical, personnel, program, and transformational goals.

The researchers conducted a two-way analysis of variance (ANOVA) and found significant main effects for both density of attention and level of abstraction with no significant interaction effects. Allison and Allison found that experience level was related to judged expertise, density of attention, and level of abstraction. In other words, the more experienced principals generally exhibited more complex schema for problem solving. However, the researchers noted that this result was heavily dependent on the inclusion of the entrant category, which exhibited the lowest group means in all three categories. When the entrant category was excluded, the results were no longer statistically significant. Moreover, there were anomalies in the data, such as the veteran principals which as a group exhibited higher levels of goal abstraction, but relatively low levels of attention to detail.

Allison and Allison (1993) concluded that the study supported the general conceptual framework of schema theory: judged expertise levels were positively related to levels of attention to detail and abstraction of imputed goals. While there was a connection between expertise level and experience, the relationship was more complex than assumed. The authors suggested that specific, domain-relevant experience (rather than experience as a measure of overall tenure) may be necessary for developing more 
abstract approaches to problem-solving, and that other contextual variables may play an important role in shaping a principal's expertise in problem-solving.

Like Allison and Allison (1993), Leithwood, Steinbach, and Raun (1993) looked at the problem-solving approaches and cognitive processes of school administrators, in this case superintendents. Leithwood et al. utilized an information processing approach and described previous research on the problem-solving practices of school administrators deemed "expert." This framework described 18 ways in which expert administrators differed from non-experts in the categories of their interpretation of problems, goals for problem solving, how they viewed constraints to problem-solving, their solution processes, their values and the role of moods and emotions in their problem-solving practices.

Leithwood et al. asked superintendents in Ontario, Canada, to nominate five of their peers they considered particularly effective. The researchers invited the 11 topranking nominees to participate in the study. Ten agreed, but three later dropped out for a variety of reasons, leaving an achieved sample of seven superintendents deemed "expert" by their peers. The researchers asked each participant to audiotape a meeting with senior administrative colleagues in which the group discussed a particularly vexing, illstructured professional problem. Later, they conducted interviews with each superintendent and played back the audiotapes of the meeting, asking the participant to comment on his or her thinking processes during the meeting. Two researchers other than the interviewer then coded interviews according to the 18 elements of the framework. In general, the researchers sought to understand what purposes were being served by the superintendent's group problem solving and how they worked to achieve 
those purposes. More specifically, the researchers wanted to know which of the 18 elements of expert problem solving these superintendents gave relatively more or less attention, and thus provide in one study a comprehensive confirmation or disconfirmation of previous research on expert problem solving.

Leithwood et al. found that the superintendents in the study did indeed exhibit approaches to problem solving deemed "expert" by previous research. Approximately 50 percent of the statements made by superintendents in the transcripts of meetings or in their comments on the meetings with interviewers focused on processes for problemsolving and exhibited the elements of expert approaches to solutions, including articulating a well-developed plan, inviting and synthesizing the ideas of others, and ensuring follow-up. Approximately 25 percent of participant statements had to do with interpreting problems in a broad context and articulating a range of goals. Another 25 percent of their statements focused on the integration of processes for both understanding and solving problems, including maintaining a positive emotional affect and engaging in self-reflection. In fact, the researchers found that self-reflection was the most frequent behavior exhibited by superintendents in the study both during and after their problemsolving situations. Leithwood et al. concluded that data supported the concept of how expert administrators utilize different cognitive processes when engaged in problemsolving.

Ruff and Shoho (2005) did not study principal "theories of practice" per se, but their study on elementary school principal "mental models" explored school leader cognitive processes in a way that parallels Argyris and Schön's (1974) concepts and suggests possible ways to operationalize theories of action for future studies. Ruff and 
Shoho (2005) conducted a qualitative investigation of three urban elementary school principals, purposefully chosen because of their relative levels of experience (one firstyear principal; one experienced principal; and one veteran, award-winning principal). The researchers drew from literature on cognitive studies of educational administration and schema analysis to support the concept of mental models, defined as "a dynamic memory system developed from previous acquisition cycles of a specific idea, activity, or role (p. 557). Ruff and Shoho sought to describe the mental models principals used to construct their roles as instructional leaders and how these mental models differed by level of job experience.

Ruff and Shoho (2005) used interviews, observations, a contrived dialectical exercise (based on Argyris, 1993), purposive observation during a faculty meeting, interviews with randomly selected teachers and a review of relevant artifacts to collect data. The researchers also conducted a pilot study to fine-tune the interview protocol and dialectical exercise. Ruff and Shoho categorized data using a constant comparative analysis technique and facilitated trustworthiness of the case studies through peer coding and member checks. Following the structure of schema analysis, the researchers identified the principal mental models of instructional leadership as consisting of an (a) perception focus, (b) standard for assessment, and (c) approach design.

Ruff and Shoho (2005) discovered that the cognitive structures that emerged in all three principal concepts of instructional leadership mirrored the structures of mental models in earlier literature. The authors found that while the "issues, conditions, routines, and words used to describe" the principal mental models were similar, the "tacit meaning each principal attached" were different (p. 571). The researchers found that a 
core set of assumptions grounded each principal's mental model for instructional leadership. The novice principal assumed he must maintain a balance between people and programs in terms of promoting student achievement; for the experienced principal, her assumption was that leadership resided within the person of the principal; and the veteran principals' assumption was that leadership was a collaborative process. These assumptions informed very different standards and assessment and approach designs to promote instructional leadership. In the cases of the novice and experienced principal, the subjects promoted relationships with staff that reinforced their superior-subordinate assumptions toward leadership, whereas the veteran principals' assumptions led to a much more open, personal and collaborative approach that invited staff members into the decision-making and leadership processes.

The specific findings of Ruff and Shoho's (2005) study are informative to the topic of instructional leadership, but their most important contribution was testing the construct of mental models for describing principals' cognitive processes vis-à-vis instructional leadership. There are parallels between Ruff and Shoho's understanding of mental models and Argyris and Schön's concept of theories of practice. Specifically, both constructs suggest that practitioner problem-solving strategies emerge from a set of values, beliefs and assumptions that may or may not be evident to the practitioner. Also, Ruff and Shoho's “approach design” seems to parallel Argyris and Schön's (1974) concept of theories of action. In short, mental models may provide a way to operationalize the way in which theories of practice are manifest in the cognitive processes of principals when they engage in instructional decision-making and leadership activities. 
In summary, the concept of reflective professional practice (Argyris \& Schön, 1974; Schön, 1983) holds great sway in the field of organizational learning, and suggests a powerful model for why professionals often engage in ineffectual behavior and how they can, with great patience and self-reflection, move to behaviors based on values of openness, trust, vulnerability and caring. Initial research indicates the models of reflective practice accurately describe the professional behavior of teachers (Ferry \& Ross-Gordon, 1998; Kirby \& Paradise, 1992; Kirby \& Teddlie, 1989; Tsangaridou \& O'Sullivan, 2000), and have promise for improving the effectiveness of principals (Erlandson, 1994; Polite, 2000). In particular, Ruff and Shoho's (2005) concept of mental models offers a promising way to describe how theories of practice work in the cognitive processes of principals.

As Day (2000) has argued, effective principal behavior may be congruent with Schön's concept of "reflection in action." This review of literature reveals that further study into the role of reflective practice and principal effectiveness for enhancing student achievement through transformed school culture are warranted.

Conceptual Framework for the Study

Two-and-a-half decades of school reform efforts, including A Nation at Risk, the federal No Child Left Behind Act, and state-level accountability initiatives such as the Kentucky Education Reform Act have led to few improvements in student outcomes (Howard, 2003; National Center for Educational Statistics, 2006). Many of these megapolicy reforms have involved both standardization of curriculum, instruction and assessment, but also decentralization of power and decision-making authority. Even these decentralizing efforts, such as the implementation of Site-Based Decision-Making 
Councils, have done little to change the overall business of schools and have led to few improvements in student learning (Björk \& Keedy, 2003; Din, 1997; Klecker at al, 1999; Leithwood \& Menzies, 1998).

The explanation for this change-resistance of schools also points the way to some possible solutions. The historic schism between theory and practice on the part of professional educators has left relationships among teachers marked by isolation, independence, mistrust and competition (Keedy, 2005; Keedy \& Achilles, 1997). Principals have been victim of this gap between theory and practice as players in the culture of isolation, and their work is characterized primarily by a focus on control of the school (Cusick, 1992). It is no wonder, under these circumstances, that schools remain resistant to change (see Figure 4).

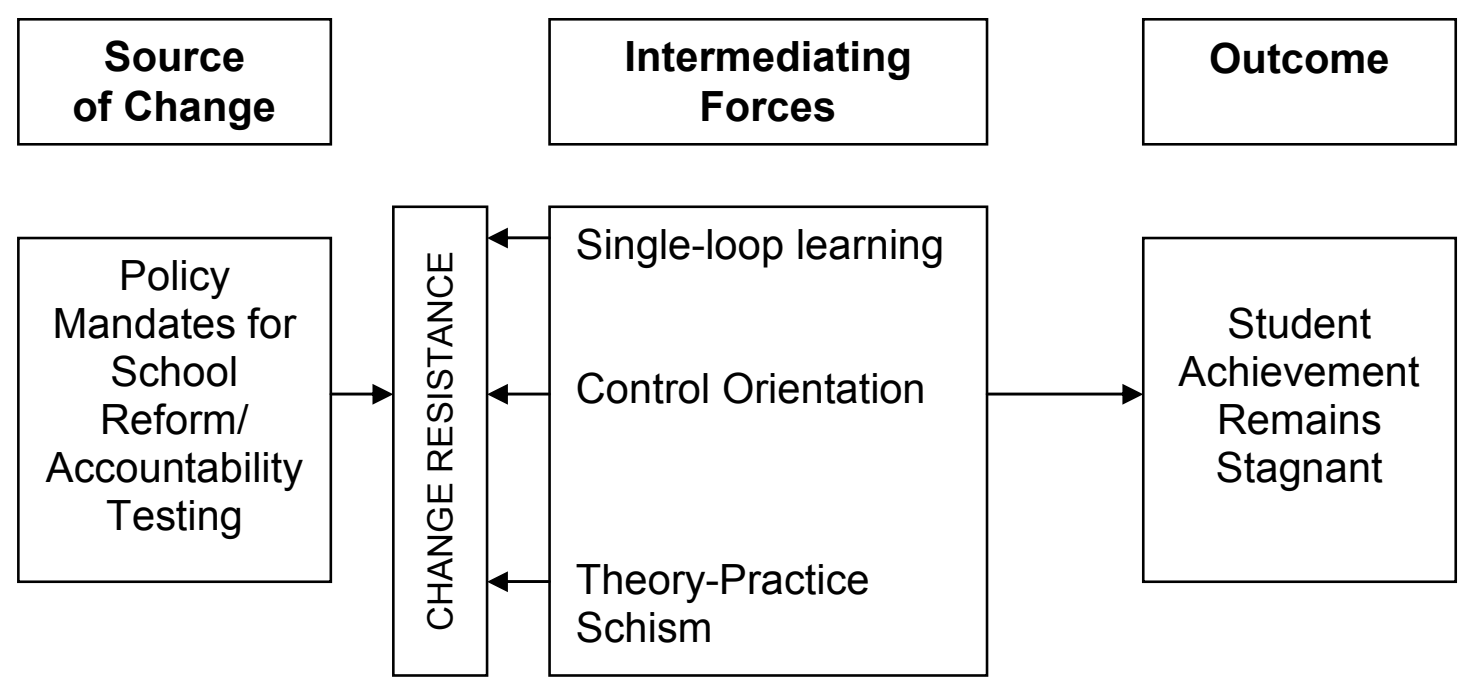

Figure 4. How policy mandates for school reform have failed to lead to higher levels of student achievement.

It is incumbent upon the principal, however, to use his or her personal and positional power to alter norms of behavior and relationships within schools to address these issues. Research indicates that principals can indeed have a positive if indirect 
effect on student achievement (Hallinger \& Heck, 1998; Marzano et al., 2005; Witziers, et al., 2003), and these effects are mediated through the principal's ability to shape relationships among school staff and the attitudes, beliefs and behaviors of teachers (Anderson, 2004; Basom \& Frase, 2004; Blase \& Blase, 1999; Short, 1994; Davis \& Wilson, 2000; Keedy \& Simpson, 2001; Spillane \& Thompson, 1997). By fostering relationships of caring, trust, collaboration, experimentation, inquiry and risk-taking, schools can potentially become centers of inquiry, rather than targets of change, and have far greater capacity for increasing student achievement (Sirotnik, 1987).

Keedy and Achilles (1997) and Keedy (2005) suggested principal-developed theories of practice as a means of creating new norms of behavior within schools. Initial empirical studies have bolstered Argyris and Schön's (1974) framework for theories of practice, and have promise for improving the effectiveness of principals (Erlandson, 1994; Ferry \& Ross-Gordon, 1998; Kirby \& Teddlie, 1989; Kirby \& Paradise, 1992; Polite, 2000; Tsangaridou \& O'Sullivan, 2000).

This study investigated the theories of practice regarding instructional leadership of four principals in successful Kentucky schools. Principals ostensibly operate according to a wide variety of theories of practice in their work, including such common issues as scheduling, staffing, budgets and financing and facilities operations. It is in the role of instructional leader that principals have the greatest impact on student achievement, mediated through their affective influence on teachers (Blase \& Blase, 1999), and for this reason the present study focused exclusively on the principal theories of practice regarding instructional leadership. 
This was the first research study explicitly describing the theories of practice of successful principals. It was also the first study to explore how principal theories of practice impact teacher perceptions and behaviors.

Principal-developed theories of practice would be one initial step toward the kind of methodology that Sirotnik (1989) suggested can increase the change-capacity of schools and heal the gap between theory/research and the actual work life of educators. Spillane and Thompson (1997) conducted research on a number of school districts engaged in adopting new instructional strategies and found that the most important variable on the reform effort's success was the willingness of school leaders to support and be actively involved in the changes. Especially important was leader support for an environment of trust and collaboration that nurtures the process of teacher learning itself:

"That is, the leaders do not learn everything they need to know and then teach their colleagues. In fact, much of the leaders' learning seems to occur in the context of their efforts to help others learn" (Spillane \& Thompson, 1997, p. 199).

If school principals and district administrators have the greatest role to play in moving schools to become centers of inquiry, they must themselves be willing to engage in self-reflection. Principals might use theories of practice to unearth the underlying values that influence their decisions as leaders and educators, and experiment with new norms and assumptions in their schools and districts, testing these new theories of action (see Figure 5). This process does not happen in a vacuum, but must, by definition, be carried out in a group context:

The [leader] should expose his [sic] goal for himself and the participants [his or her co-workers] to design environments that produce learning of the model-II concepts and behavior and encourage continual confrontation of the model-II concepts. (Argyris \& Schön, 1974, p. 111) 


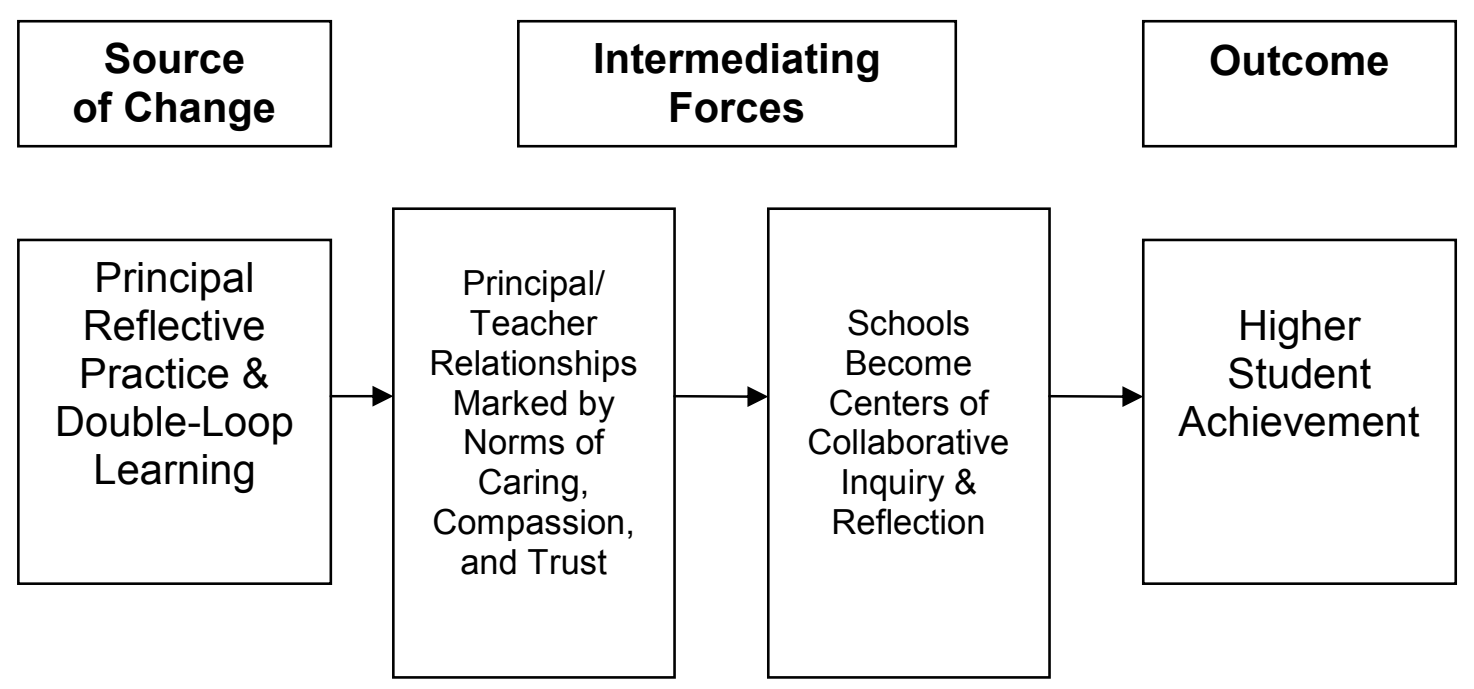

Figure 5. How principal reflective practice and double-loop learning may contribute to higher levels of student achievement.

Continual confrontation is risky and challenging but ultimately transformative.

As the entire work community becomes involved in this new approach to learning, relationships begin to change. Keedy and Achilles (1997) argued that this shift toward a more collaborative, power-sharing model of inquiry and discovery is the best measure for whether normative thinking is actually changing in the school.

New assumptions about how their organizations should work grounds a staff's shared meanings about revitalized student-teacher-principal relationships (normative consensus)...In sum, teachers and principals theorize that taking actions through changing norms maximizes a reasonable likelihood of improving school relationships through changing the norms (Keedy \& Achilles, 1997, p. 8).

Spillane and Thompson's study (1997) confirmed that effective leadership for building change capacity emerged from work relationships marked by collaboration and especially by trust:

Trust was crucial because it facilitated conversations about instructional reform. Trust was also essential for genuine collaboration among educators, enabling them to work together to develop a shared understanding of the reforms. Moreover, trust created an environment in which local educators were comfortable discussing their understandings of and reservations about new 
instructional approaches, conversations that were essential for reconstructive learning. (Spillane \& Thompson, 1997, p. 195)

Leaders may then begin to measure the impact of their theories of practice, at least in part, by their impact on student achievement, and especially on the power relationships among teachers, students, parents and their administrators. Such a change in relationships is fundamental to Sirotnik's (1989) idea of schools as the centers of change/centers of inquiry, and by Keedy and Achilles's (1997) estimation, the best measure of a genuine shift in normative thinking among educators. 


\section{CHAPTER III}

\section{METHODOLOGY}

The purpose of this study was to examine the theories of practice toward instructional leadership of four principals in consistently-improving Kentucky schools. These principals presided over increases in student achievement on the Commonwealth Accountability Testing System (CATS) at the same school over a two biennia (four-year) period. Student scores at the schools met or exceeded the state-mandated goals for improvement for each biennium. These principals were also identified by area educational leaders as possessing the qualities of reflective practitioners (Schön, 1983; 1987). Three research questions framed the study:

1. What are the theories of practice of instructional leadership for these four successful school principals?

2. How do the principal theories of practice regarding instructional leadership influence the attitudes, beliefs and behaviors of teachers?

3. To what extent does their use of theories of practice conform with Argyris and Schön's conception of double-loop learning and Schön's conception of the "Reflective Practitioner?"

A study of reflective principal leadership in high-performing schools may shed light on the specific theories of practice that contribute to student success. 


\section{Design}

This study investigated the theories of practice of successful school principals in Kentucky. According to Argyris and Schön (1974), theories of practice are deeply embedded in the psychology of the professional practitioner, and include values, beliefs and assumptions of the practitioner, as well as theories of action for how to accomplish goals in specific work-related situations. Thus, theories of practice are unique and personal to each practitioner and are context-specific. Additionally, Argyris and Schön found that practitioners' actual theories-in-use were often at odds with their espoused theories, and so this incongruence makes it difficult to ascertain an individual's theories of practice simply by asking. For all these reasons, this study used a qualitative approach of naturalistic inquiry (Lincoln \& Guba, 1985).

A naturalistic design is better suited for studying theories of practice because it allows the researcher to observe the principal's behaviors in the particular school context corresponding to those theories of practice. Previous qualitative studies demonstrated the usefulness of a naturalistic approach for studying these deeply personal and contextspecific dimensions (Day, 2000; Ferry \& Ross-Gordon, 1998; Polite, 2000; Rogers, 2004; Ruff \& Shoho, 2005; Tsangaridou \& O’Sullivan, 2003). Through interviews and reflective activities with principals and teachers, observation, document analysis and thick, rich description, a more complex picture of the principal's theories of practice and the specific outcomes of those theories emerged.

This study used a multi-case study design so the researcher could closely examine the theories of practice within the specific contexts of four schools, and how principal theories of practice influenced teachers. According to Rossman and Rallis (1998), the 
case study approach allows the researcher to "understand a larger phenomenon through close examination of a specific case and therefore focus on the particular" (p. 70). Multicase studies afford the researcher the opportunity to examine phenomena as they appear in a variety of contexts (Miles \& Huberman, 1994). The case study provides the best means of inquiry for exploring the unique and particular theories of practice of individual principals in specific contexts.

\section{Participants}

This study used a purposeful sample. Merriam (1998) described purposeful sampling as "based on the assumption that the investigator wants to discover, understand, and gain insight and therefore [the researcher] must select a sample from which the most can be learned" (p. 61). Purposeful sampling "increases the scope or range of data exposed ... as well as the likelihood that a full array of multiple realities will be uncovered" (Lincoln \& Guba, 1985, p. 40). High-stakes accountability systems, like Kentucky's, provide a measure of school progress over time. This study investigated the role of principal theories of practice regarding instructional leadership in such consistently improving schools. This study, therefore, targeted school principals whose schools met or exceeded their state-established targets for improving student achievement during a two-biennia period and who were also described by area educational leaders (superintendents, educational administration professors, and staff members at the area educational cooperative) as possessing the characteristics of a reflective practitioner. Four cases allows for in-depth cross-case analysis of data from multiple sites while limited the number of cases to allow for a rich exploration of each school context. The case, or unit of analysis, was each principal and his or her school. 
The researcher utilized the nomination technique (Hunter, 1953) to delineate principals who area educational leaders believed possessed qualities of a reflective practitioner. The researcher administered a questionnaire to a regular monthly meeting of superintendents, district administrators and education administration professors at the Red River Regional Educational Cooperative (RRREC; pseudonyms used throughout the study). The researcher described the nature of the study, and administered a questionnaire asking participants to identify principals from the RRREC region who met the criteria of a reflective practitioner (see Appendix A).

To be considered for this study, principals must have worked in schools that met or exceeded the state-established goal for improving student achievement during both the biennium ending in 2006 and in 2004. Kentucky's Commonwealth Accountability Testing System (CATS) utilizes an academic index that reflects each school's progress toward proficiency. The academic index ranges from a score of 0 to 140 , with a score of 100 indicating that almost all students in the school are performing at a proficient level as measured by CATS, which includes both norm- and criteria-referenced testing components and measures of non-academic achievement such as attendance rates and retention. A formula determines the target academic index. The formula considers the school's most recent level of achievement and the requisite increments of improvement required for that school to reach an academic index of 100 by the year 2014. So that no single year's results skew the appearance of progress, the increments are established based on the combination of test scores from two years, ending in even-number years. For sample selection for this study, the researcher reviewed CATS results available on the Kentucky Department of Education Website and identified schools within the 34 
districts comprising the Red River Regional Educational Cooperative (RRREC) that had met or exceeded their target academic index in the biennia ending in 2004 and 2006. Next, the researcher determined which of the identified schools were led by the same principal during the school years comprising the 2004 and 2006 biennia to generate an initial list of potential participants. The researcher then compared this list with principals nominated for the study by RRREC superintendents and other area educational leaders. A pool of eleven eligible participant nominees emerged.

The researcher then contacted these principals by e-mail to describe the study, to inform the principals that they were nominated as participants, and to inquire as to whether the principal would be willing to be interviewed by phone for possible selection for the study. Six principals who indicated interest were contacted by telephone for a brief interview utilizing the same questions above. The researcher further narrowed the list based on principal responses to the interview questions. Finally, the researcher purposefully chose four principals to represent rural, urban and mid-size communities, and to include both male and female participants. Principals willing to participate in the study provided the researcher the names of teachers who had worked in the school during the 2004 and 2006 testing biennia. The researcher randomly selected 12 teachers from this pool (three from each school) who were interviewed as to the principals' instructional leadership beliefs and behaviors.

\section{Gaining Entry}

The researcher submitted the proposed study to the Institutional Review Boards of both the University of Louisville and Western Kentucky University prior to any contact with potential participants. Approval of the Boards indicated that the proposed study 
poses no major risk to participants. The researcher submitted verification of his Collaborative IRB Training Initiative (CITI) certification to both Institutional Review Boards as part of the proposal review process.

The researcher sent an introductory e-mail to each potential participant principal informing him or her of the proposed study and that a formal, detailed introductory letter would be mailed providing further information. The introductory letter and consent form outlined the purpose of the study and solicited the principal's participation. The researcher sent a follow-up e-mail to confirm the principals' receipt of the letter and to answer questions about the study. After gaining permission and collecting the consent form the researcher contacted the principal to begin data collection and to obtain the names of potential teacher informants for the study. The researcher selected potential teacher participants according to the same protocol as principals: (a) an introductory email, (b) a formal letter describing the study and seeking the teacher's consent to participate, (c) a follow-up e-mail to confirm receipt of the letter, and (d) a call or e-mail to arrange for interviews and data collection. The researcher purposefully selected other teacher informants as the data collection proceeded.

\section{Data Collection}

Data collection consisted of interviews with the principals and teachers, naturalistic observation, and a contrived reflective exercise.

\section{Interviews}

The researcher conducted semi-structured interviews with the principal and teacher informants. 


\section{Questions}

Effective interview questions should assist the researcher in answering the research questions (Merriam, 1997). The researcher used the research questions for this study as a guide to develop interview protocols. The framing questions for the study included the following:

1. What are the theories of practice of instructional leadership for these successful school principals?

2. How do principal theories of practice regarding instructional leadership influence the attitudes, beliefs and behaviors of teachers?

3. To what extent does their use of theories of practice conform with Argyris and Schön's conception of double-loop learning and Schön's conception of the "Reflective Practitioner?"

\section{Principal interview}

The researcher interviewed each of the principals on multiple occasions. The first interview was conducted at the beginning of the study and focused on the following questions:

1. Please describe your school (collect data regarding setting here).

2. Please tell me about your career and your personal background (collect data regarding biographical information here)

3. How do you understand your role as instructional leader of this school? What does that role mean for you?

4. What are some of your deep, fundamental assumptions about teaching and learning? 
5. In what ways do you engage in intentional instructional leadership at this school?

6. What has been the result of your instructional leadership efforts? What has worked and why? What has not worked and why?

7. Can you describe an instructional leadership strategy you used that did not work? Why do you think it failed? What did you do or will you do differently (if anything) as a result of that failure?

8. When and in what ways could I observe you engaging in instructional leadership activities?

From this initial interview, the researcher developed tentative statements describing the principal's theories of practice toward instructional leadership. These were shared with the principal for clarification, feedback and discussion. The researcher interviewed the principal repeatedly throughout the data collection process to ask clarifying questions and to conduct member checks of the patterns that emerged from interviews with the principal and teachers and observation. Because each principal's theories of practice were unique, protocols for the follow-up interviews depended on data emerging as the study progressed.

The researcher recorded interviews using a digital audio recorder and took written notes. The researcher transcribed the data and asked the principals to review the data for accuracy and clarity. All school identifiers and the principal and teacher names were removed and pseudonyms were assigned to the data. In the narrative of results, interviews are cited by noting the initials of the interview and the date of the interview. For example, (ME, 11/22). 


\section{Teacher interviews}

Participating principals provided the researcher a list of teachers with at least five years of experience in the same school. The criterion of five years ensured that teacher informants had worked with the principal during at least two biennia of increases in student achievement and had developed well-informed perspectives on the principal's theories of practice. A random sample of three teachers was taken from the list for interviews. Additional teacher informants were purposefully selected as data collection proceeded. The teachers were informed of the nature of the study and signed a consent form to voluntarily participate. The researcher assured teacher confidentiality and assigned a pseudonym to the data. Questions guiding the initial teacher interview included the following:

1. What is the principal's chief role in this school? What is his/her main function?

2. What are his/her beliefs about teaching and learning? How, specifically, do you know this?

3. What are some of his/her instructional leadership behaviors? How do these behaviors impact the way you feel, the way you think, and/or the way you teach?

4. Have you ever observed the principal use an instructional leadership behavior that didn't work? Can you describe it? How did he/she react or do you think he/she will react to that failure?

5. Is there anything else you want to tell me about your principal's instructional leadership?

The researcher conducted follow-up interviews with teachers for clarification or to question the teacher about new information emerging in the course of the study. As with 
the principal interview, the researcher recorded interviews using a digital audio recorder and transcribed the data. The researcher conducted member checks with several teachers from each school to review the data for accuracy and clarity.

\section{Naturalistic Observation}

The researcher observed the principal as he or she conducted his or her daily routine, with special attention for times when the principal intentionally engaged in instructional leadership behaviors (facilitating faculty or team meetings, observing classrooms, conferencing with teachers, etc.). Observations were recorded using field notes, including a narrative of events and also the researcher's thoughts, impressions and connections to data obtained earlier in the study. The researcher transcribed the data. In the narrative of results, observations are cited by noting the date of the observation. For example, (Observation, 11/22).

\section{Reflective Exercise}

Argyris and Schön (1974) and Argyris (1993) described a variety of reflective exercises they used with students in consulting seminars. These exercises encouraged participants to reflect on their professional practice in a specific context. Data from the exercises were used to ascertain features of the subject's theories of practice, including espoused theories, theories-in-use and underlying values, beliefs, and assumptions. Ruff and Shoho (2005) used a reflective exercise based on those developed by Argyris and Schön to investigate the mental maps of instructional leadership of three elementary school principals. This study used the same exercise to assist the researcher in understanding participant theories of practice and ways in which they engage in single- or double-loop learning. 
The researcher asked the principal to recall a post-observation conference with a teacher whose performance was unsatisfactory. Then, on paper divided into two columns, the principal wrote out the conversation that took place on the right side of the paper, as close to verbatim as possible. On the left side of the paper, the principal wrote his or her thoughts underlying the conversation—what the principal was thinking or feeling at the time of the conversation, and what the principal now makes of the conversation in retrospect. The narrative data from this exercise was analyzed and coded. The researcher changed all names to protect the confidentiality of subjects named in the narrative.

Three of the four case study principals agreed to participate in this reflective exercise. The fourth principal, Betsy Master (Case Study D), declined to participate, stating that it had been years since she had observed a lesson that did not go well, and would not be able to complete the exercise faithfully. To compensate for the loss of this data source, the researcher used interviews to engage the principal in self-reflection about her theories of practice and evidence of double-loop learning and reflection-in-aciton.

\section{Data Analysis}

The researcher used an inductive inquiry approach that transformed "raw units of information to subsuming categories of information" (Lincoln \& Guba, 1985, p. 203).

\section{Constant Comparative Analysis}

Glaser and Strauss (1967) developed the method of constant comparative analysis to provide a scientific protocol for inductively deriving new theories or explanations of research topics. One method of developing grounded theory is the constant comparative analysis technique, in which the researcher codes data into categories in an iterative 
process. Each new datum is categorized, but the categories themselves are continuously reviewed in comparison with the new data, in an ongoing process that enables the researcher to discover inductively new patterns and explanations for the phenomena being investigated.

While constant comparative analysis is rooted in the tradition of grounded theory, qualitative researchers of all persuasions use the method for inductive inquiry (Merriam, 2001). Ruff and Shoho (2005), for example, used the technique for data analysis in their study of principal's mental maps of instructional leadership. The researcher used constant comparative analysis at all stages of the data-gathering process. Miles and Huberman (1994) recommend continuous coding and analysis throughout data collection. "It helps the field-worker cycle back and forth between thinking about the existing data and generating strategies for collecting new, often better data...we advise interweaving data collection and analysis from the start" (p. 50). Following this recommendation, the researcher utilized marginal coding, a reflexive journal, contact summary forms, and memoing as methods of constantly reflecting on the emerging analytic categories presented by the data.

\section{Within-Case Analysis}

Within-case data analysis involves focusing first on data from each case separately (Merriam, 1998). Utilizing the constant comparative technique, the researcher categorized data and identified patterns until no new categories could be generated. Data for each separate case was triangulated through multiple sources: principal interview, multiple teacher interviews, naturalistic observation notes, and from the principal 
reflective exercise. Within-case data were displayed through visual representations of each theory of practice based on the conceptual framework (see Figure 6).

ASSUMPTIONS

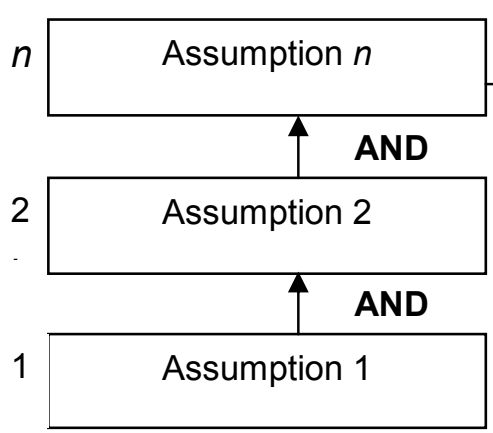

ACTION STRATEGIES

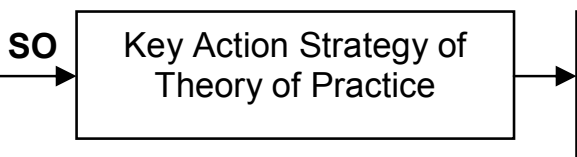

Examples of Action Strategies 1.

2.

3.

\section{TEACHER EFFECTS}

\section{Effect on Teacher's} Feelings, Attitudes or Behaviors

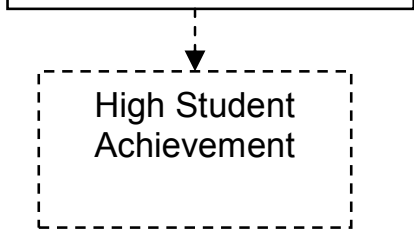

Figure 6. Visual representation for the presentation of each principal theory of practice, including the principal's core assumptions about instructional leadership, and the action strategies that logically emerge from those assumptions, impacts teacher attitudes, feelings, and behaviors. A tacit assumption of all instructional leadership theories of practice is that these action strategies will indirectly result in higher student achievement, though this link is not explored in this study (indicated by dashed lines in the figure).

\section{Cross-Case Analysis}

After analysis of each individual case, the researcher conducted a cross-case analysis, again utilizing the constant comparative technique. "A qualitative, inductive, multicase study seeks to build abstractions across cases" (Merriam, 1998, p. 195). The researcher created matrixes to summarize and display data across cases (Miles \& Huberman, 1994). Emergent categories that explain patterns across cases were identified in a continuous way until data saturation occurred (Glaser \& Strauss, 1967). Merriam (1998) noted that this process involves both inductive and deductive inquiry, in that the researcher begins to develop hypotheses about regularities and patterns within the data, and looks for information across the cases to confirm or disconfirm these tentative hypotheses. A kind of holistic picture of the phenomena under study begins to emerge, 
"transcend[ing] the formation of categories ... seek[ing] to explain a larger number of phenomena and tell how they are related" (Merriam, 1998, p. 192).

\section{Trustworthiness of the Data}

For qualitative research, trustworthiness is the extent to which a reader experiences the conclusions of a research study as believable and real for subjects in the particular context being portrayed (Lincoln \& Guba, 1985). Dimensions of trustworthiness include the credibility, transferability, dependability and neutrality of the overall study. The following methods contributed to the trustworthiness of this study.

\section{Triangulation}

Data emerged from multiple sources within this study. The researcher interviewed principals and teachers more than once. Data also emerged from naturalistic observation, and from the principal reflective exercise. Drawing data from multiple sources helped establish the researcher's efforts to convey the perspectives and experiences of the participants accurately (Rossman \& Rallis, 1998).

\section{Member checks}

Lincoln \& Guba (1985) identified member checks as a means of combating researcher bias and contributing to the trustworthiness of qualitative conclusions. The researcher showed transcripts of interviews and emerging conclusions about overall patterns in the data with participants for accuracy and clarification, and made adjustments based on feedback from the subjects.

\section{Thick, rich description}

The strength of qualitative research, according to Rossman \& Rallis (1998), is that by painting a detailed picture of a case, the reader can draw his or her own conclusions 
about results. Data from interviews, observation and the reflective exercise were conveyed in a rich narrative.

Reflexivity

Finally, the researcher combated his own bias by engaging in continuous reflexivity during the data collection process. The researcher maintained comprehensive research notes where he observed his own thinking and analysis processes as they occurred, made new connections, and was mindful of subjective interpretations of the data.

\section{Limitations}

The chief limitation of this study, as in most qualitative research, was the small number of participants (four Kentucky principals and their schools), which limits generalizability. Miles and Huberman (1994) noted that this is typical of case study designs, since "qualitative researchers usually work with small samples of people, nested in their context and studied in-depth" (p. 27). This in-depth nature of qualitative research provides a richness of description that offsets limitations of generalizability due to the small number of cases in the sample. 


\section{CHAPTER IV}

\section{RESULTS}

Four principals served as research subjects for this study. Data from each case are presented below. The researcher used the following methods to gather data for each case: (a) multiple interviews with the principals, (b) multiple interviews with three teachers per principal, randomly selected from a pool of teachers who worked under the principal's leadership for at least five years, (c) approximately ten hours of naturalistic observation of the principal at work, and (d) a self-reflective written exercise completed by three of the four principals (the principal in Case Study D declined to complete this activity; see subsection for this case study). The researcher recorded and transcribed all interviews and observation notes and used constant comparative analysis to analyze the data to the following research questions:

1. What are the theories of practice of instructional leadership for these four successful school principals?

2. How do principal theories of practice regarding instructional leadership affect the attitudes, beliefs and behaviors of teachers?

3. To what extent does their use of theories of practice conform with Argyris and Schön's conception of double-loop learning and Schön's conception of the "Reflective Practitioner?"

Each case study includes a description of the research subject and the school context. For Research Question 1, the researcher identifies the key theories of practice 
that emerged from the data in describing the principal's approach to instructional leadership, including the underlying assumptions that provide a conceptual foundation and the action strategies that logically emerge from those assumptions for each theory of practice. For Research Question 2, the effects of the theories of practice on teachers are described. Figures present a visual display of data for each theory of practice and its effects. Finally, for Research Question 3, whenever data indicated the principal engaged in double-loop learning or reflection-in-action, the researcher describes that process as well. Double-loop learning is the cognitive process by which a practitioner uses feedback to reflect on his or her theory of action, and then questions and revises the underlying assumptions rather than simply adjusting their action strategies to achieve a new outcome.

\section{Case Study A}

Marie Edmonds: Attending to the Individual

Marie Edmonds, principal of Cane Ridge Elementary School (CRES; pseudonyms used throughout the study) for the last 12 years, was a 30-year veteran of education. Cane Ridge was the first and only principalship for Edmonds, who held an Ed.D. in educational leadership. She was a former elementary school teacher and gifted and talented education (GTE) teacher who spent several years working as a consultant for a university GTE foundation prior to earning her doctorate and becoming principal. She encouraged staff to call her "Ms. Edmonds" rather than "Dr. Edmonds" because she believed it was less confusing for students. Ms. Edmonds's theories of practice of instructional leadership emerged from key assumptions about the responsibilities of 
educators to respond to the unique needs of the individual student and the contribution of strong personal relationships to student and teacher success.

\section{School Context}

Cane Ridge Elementary, part of a county district of 12,000 students, served 430 children in kindergarten through sixth grade. The brick school building sat just off a major commercial thoroughfare and adjacent to the interstate highway that bisected the county. Cane Ridge's staff of 37 teachers served students from both affluent suburban and low-income neighborhoods surrounding a city of approximately 50,000 in South Central Kentucky. The student population included 55 percent on free or reduced lunch, and 15 percent minority students. The school identified approximately $8 \%$ of students as English language learners. Like the larger community, CRES's student population had become more diverse with a large influx of families including Hispanics, Bosnians, Koreans and Japanese. Due to rapid growth in the community, the school experienced redistricting several times in recent years. From a student population of 630 at its peak, CRES dropped from 580 to 430 students in the year just prior to this study. The district reassigned eight teachers to other schools and 60 new students enrolled at Cane Ridge.

Despite the redistricting challenges, the school had a long record of improving student learning as measured by the Commonwealth Accountability Testing System (CATS), Kentucky's statewide student achievement program. By state policy, all schools must achieve an academic index of 100, indicating that almost all students are at proficient levels of performance in core subject areas, by 2014. The state measures schools' progress in two-year increments and Cane Ridge's academic index rose from 77.7 in the biennium ending in 2002 to 91.1 in 2004 and 93.2 in 2006. Edmonds credited 
her staff's collaborative decision-making and common focus on improvement for the steady gains. However, she emphasized that her school also seeks to address student needs beyond those measured by the statewide test, noting programs that promoted the artistic, cultural and social needs of children. Examples included a school-wide antibullying program, numerous multicultural speakers and events, and a program that provided instruments so students could learn music and participate in a string ensemble regardless of their financial ability to pay for instruments or lessons. Edmonds believed these programs were just as important as the statewide testing goals. She would not compromise them, even though they added nothing to the school's academic index:

CATS scores are not the most important thing at Cane Ridge. I think in some places, kids and teachers are almost beat over the head with it. That won't ever be the case as long as I'm here. I always tell the teachers, we are going to work as hard as we can to help children learn and I agree that there are some tricks to the trade, that we can teach kids how to take the CATS test and that we are doing them a disservice if we don't teach them some of that. We're just not going to beat them over the head with it. (ME, 9/25)

Teachers at Cane Ridge shared this concern for meeting the needs of the whole child, and responded favorably to Ms. Edmonds's emphasis on personal relationships with students and staff.

\section{Case Study A: Research Findings for Marie Edmonds}

Five theories of practice described Marie Edmonds's instructional leadership. Subsections below describe each theory of practice, including the core assumptions that made up the foundation of the theory of practice, the action strategies that logically emerged from those assumptions (Research Question 1), and the effects of the theory of practice on teachers (Research Question 2). When data suggested Edmonds had engaged 
in reflective practice by adjusting or modifying her theories of practice by revising her assumptions or action strategies, this process is also described (Research Question 3). Theory of Practice 1: Meeting Individual Student Needs

A dominant feature of Ms. Edmonds's leadership was an emphasis on meeting each child's unique emotional, social, and cognitive needs. For children to succeed in school, teachers must be sensitive to these needs and respond accordingly. Edmonds perceived her role as principal to include communicating her vision of student-centered learning to teachers, modeling positive personal relationships with students, and intentionally organizing school activities to support individual student success. As a result, teachers shared her commitment to the individual child and responsibility for student success (see Figure 7).

Assumptions. Throughout the interviews and observations conducted for this study, a clear emphasis on the "individuality" of each student emerged in Ms. Edmonds's instructional leadership. Schools must consider the unique elements of each child's life, including learning style, gifted or disability status, socio-economic status, ethnicity and aptitudes. Edmonds's assumptions about a high-quality education individualized for each child originated from her experience as a gifted and talented teacher (ME, 9/25; JJ, 10/16). For children to succeed in learning, schools must be sensitive to the child's unique needs and respond accordingly, and when these needs are met students will succeed academically. Her role as principal included communicating her vision of learning, modeling positive relationships with students, and organizing school activities and guiding teacher practices to be intentionally responsive to individual student needs. 
ASSUMPTIONS

\begin{tabular}{|c|c|}
\hline 4 & $\begin{array}{l}\text { Schools aren't naturally } \\
\text { sensitive or responsive } \\
\text { to individual student } \\
\text { needs }\end{array}$ \\
\hline & AND \\
\hline \multirow[t]{2}{*}{3} & $\begin{array}{l}\text { When students feel } \\
\text { affirmed and valued and } \\
\text { when their individual } \\
\text { needs are met, they will } \\
\text { succeed academically }\end{array}$ \\
\hline & AND \\
\hline \multirow[t]{2}{*}{2} & $\begin{array}{l}\text { For children to succeed } \\
\text { in learning, schools must } \\
\text { be sensitive to these } \\
\text { unique needs and } \\
\text { respond accordingly }\end{array}$ \\
\hline & AND \\
\hline 1 & $\begin{array}{l}\text { Individual children have } \\
\text { unique emotional, social } \\
\text { and cognitive learning } \\
\text { needs }\end{array}$ \\
\hline
\end{tabular}

ACTION STRATEGIES

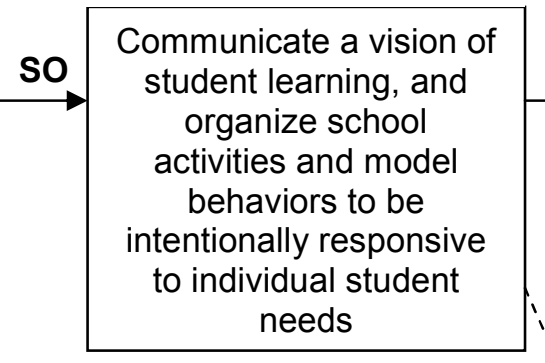

1. Constantly communicate the belief that all students can learn if their needs are met.

2. Model that vision for students and teachers

3. School GTE program

4. Special education

5. PTO strings ensemble program

6. Multi-cultural focus.

7. Include valuable subjects in student learning regardless of whether the subject appears on the state test

\section{TEACHER EFFECTS}

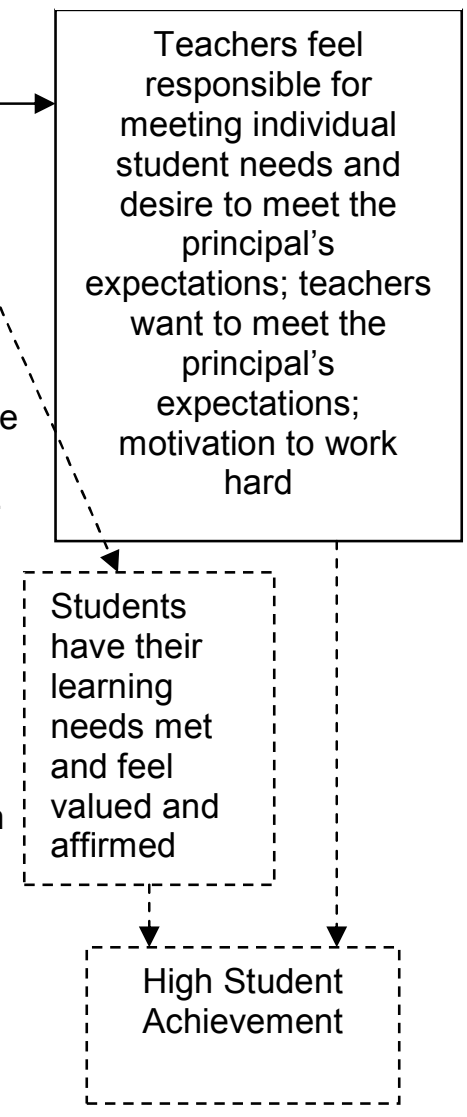

Figure 7. Marie Edmonds, Theory of Practice 1, for meeting individual student needs, including assumptions, action strategies and teacher effects. A tacit assumption of all instructional leadership theories of practice is that these action strategies will indirectly result in higher student achievement, though this link is not explored in this study (indicated by dashed lines in the figure).

Teachers at Cane Ridge confirmed Edmonds's emphasis on the individual child and indicated that they had embraced the same assumptions. "Students come first... [and] all students should be challenged, not just the gifted students but all students and our role is to make sure that all of this happens in the best way possible," according to Darla Hammond, the school's elementary curriculum coordinator (ECC) who worked with Ms. Edmonds for 11 years (DH, 10/16). 
"She's always ready and encouraging" in response to special student needs, according to Julie Jones, who taught special education for 11 years under Edmonds's leadership. '[She says] 'Let's keep going. What more can we do?' We've got this child that is non-verbal and she's sending me to something [a training seminar] later this month. How can we help his child be able to be in the regular classroom and be more independent?" (JJ, 10/16).

Daniel Baker, a math teacher at Cane Ridge for 10 years, echoed Jones's opinion: Ms. Edmonds tried to be "sensitive to that [individual student needs] and she tries to help us be sensitive to that in terms of the amount of patience it requires, but as well as the fact that we need to keep the bar up for everybody" (DB, 11/20).

According to Edmonds, effective teaching depends on establishing strong interpersonal connections with students:

It's our job as educators to get to know our children well enough through talking with them, spending time with them, as well as through all of the formative and summative assessments that we have available to figure out how they learn best, how they need to be taught, what supplementary intervention and support they need. (ME, 9/25)

This responsibility is especially important to her as an elementary principal. Edmonds's own sense of responsibility originated from her belief in the power of a principal to shape school culture. She discovered this potential for principal effectiveness when, prior to becoming a principal, she worked on a grant helping elementary schools implement a multiage primary program:

We'd go into schools, we'd do model lessons, we'd work with teams of teachers to develop curricula, and then we would come back a month or two later and some of the schools were implementing and in some schools it was like we'd never been there. As we would reflect on what's the difference, it was the principal. The leadership set the tone. ... I began to think that's a way you can really make a difference for children. (ME, 9/25) 
Action Strategies. Data revealed numerous examples of Ms. Edmonds's efforts to communicate her vision of a student-centered learning community to teachers, to model positive relationships with students, and to organize the school's activities in a way that promoted student self-esteem and achievement. She called it "one of my most important roles.... To me it means setting the tone and philosophy of the school that all children can learn at high levels" (ME, 9/25).

Ms. Edmonds consistently communicated her core instructional vision for the school at every occasion, according to Ms. Jones:

She just wants every child — every child — to be successful. At almost every faculty meeting she conveys that to us. This year we have a high-risk population, but that has not deterred her from the belief that we're going to help each one of these children be successful, despite what they go home to. (JJ, 10/16)

Ms. Hammonds confirmed that Edmonds regularly conveyed this positive, "can do" attitude (9/25) regarding the responsibility of staff for student learning and the possibility for high student achievement. "I see her as the major cheerleader and instigator of what's best for kids. It may not be best for me personally, but that's not why we're here:"

We have our mission statement and we talk about it at faculty meetings.... We talk about it when we do our professional growth plans. We talk about it when we look at our CSIP [Comprehensive School Improvement Plan]. Any decision that is made, you know the question is going to be asked, "What's best for the kids?" That helps decide a lot of things when it comes down to personal views and preferences. It takes away "I think." (DH, 10/16)

Hammond, who worked closely with Ms. Edmonds in her role as curriculum specialist, was also the school's assessment coordinator. She affirmed that Edmonds's emphasis was broader than promoting higher test scores. "We've had several conversations [about ways to improve test scores], especially after you go to the 
assessment conference, and you come back with 1,999 different ways to prepare for assessment. And we'll say, 'You know, we don't want to be that kind of school'" (DH, 10/16). The staff still did what they could to improve student performance as measured by CATS, but this was not the school's primary focus:

If it comes down to, we can't do this program because it's not test-related, but we feel like it's really good for the kids just to make them a better person, we're going to do it. . . .If you make a well-rounded child, eventually that's going to turn into good test scores too. . . Are we top in the district? No. Would we like to be? Sure. But are we going to give up some things to get there? Probably not. (DH, 10/16)

In addition to communicating her assumptions about student learning to teachers, Edmonds actively made efforts to celebrate individual student achievements and organize school events to promote student diversity and address individual student needs. During a faculty meeting, she asked teachers to share with her the names of students who earned proficient scores on open response writing tasks (Observation, 10/24). On another occasion, she walked through the hallways looking at displays of student work and recorded the names of students with proficient scores so she could recognize them at the school's morning meeting (Observation, 11/7). At school-wide meetings each morning, Edmonds celebrated individual students who had demonstrated significant academic achievements (ME, 9/25; Observation, 10/31).

This attention to individual students was not a new emphasis for Edmonds, according to Ms. Hammond:

We celebrate successes. We haven't done it as much this year because we've been splintered a little more in our [faculty] meetings, but we'll start a meeting with sharing something successful that happened in our classrooms. It may just be something like, a child read three words today, and we'll all celebrate that. (DH, 10/16) 
Edmonds's emphasis on the individual child extended to addressing the students' cultural and socio-economic needs. The school's Parent-Teacher Organization provided scholarships for underprivileged students to participate in the school's string ensemble (ME, 9/25). Ms. Jones related the principal's pursuit of and support for a federal Reading First grant to her concern for special needs students (JJ, 10/16).

According to Edmonds, the diversity of the student body presented another opportunity to celebrate and recognize the unique ethnic backgrounds of many of the children:

Cane Ridge is a slice of American life because we have that diversity which supplies a very rich educational experience for our children. We wrote a grant a few years ago called "A Celebration of Culture." It was an arts and humanities and foreign language grant and ... through that we've had lots of visiting artists. We've tried to bring in Hispanic singers and dancers, African American drummers, so that each child feels their culture is truly celebrated. (ME, 9/25)

The school also employed a full-time Spanish teacher, the only elementary school in the district to do so (ME, 9/25).

Her concern for individual children's needs also appeared in the school's bullying prevention program. A local kung fu teacher, "Master Bob," regularly visited the school and gave presentations to the students on self-confidence, responding to bullies and treating one another with respect. "I hope it's the kind of culture that kids will come to me or a teacher if they are being bullied and not be afraid," Edmonds explained (ME, 9/25).

Ms. Edmonds informally discussed a student's referral for special education services with teachers, and stopped for a lengthy conference with a student in the hallway whose teacher removed her from the room for misbehavior. Edmonds spent twenty minutes helping the child with her math assignment before discussing her classroom 
behavior. The child reported that she was "mad at the world," and described a

tumultuous home life involving an at-risk older brother and the difficulties of living with two separated parents. Edmonds coached the girl on ways to address some of her problems at home, encouraged her to talk to the guidance counselor, and then assisted her in writing an apology note to the teacher for her misbehavior (Observation, 11/7).

Teachers described Edmonds's willingness to take on any role or task, no matter how mundane, as an example of her own level of personal responsibility for student success. Edmonds was coaching the academic team at the time of the study because no coach was available and she did not want to see the team disbanded. The school sponsored a program called "Check and Connect" in which identified at-risk students maintained an individualized check sheet designed to assist them with addressing work habits or other behaviors that support academic success. The school counselor visited with these students on a daily basis to review student progress, but when the number of students became too many for one person, Edmonds took on two students herself, and visited with them at either the beginning or end of each school day (Observation, 10/31).

Ms. Jones indicated that Edmonds was willing to take on a broad range of roles:

If need be ... she's the substitute teacher, the cafeteria line worker, the parent consultant, the ARC [special education Admission and Release Committee] chair. She sits in on most of our meetings. I don't know that all schools-I think that counselors do much more of the meetings, but she's there every time. . . I almost view her as a servant leader, in that I don't think there's anything she would ask us to do that she wouldn't do herself. (JJ, 10/16)

"She leads by example," Jones continued. "She does have high expectations for us, but she's here until—I sometimes work late, but no one can work more than what Ms. Edmonds does. I've gotten e-mails from her past midnight. She's a workaholic" (JJ, 
10/16). But her model takes more forms than just the number of hours she works, according to Ms. Hammond:

She models good instructional strategies in her meetings. She uses a lot of Thoughtful Classroom strategies to show teachers this is the way to do it, also to show, "I know what they are too." . . . So when she comes to my classroom I know that she's looking for that and she knows what she's looking for. (DH, 10/16)

Mr. Baker talked at length about Edmonds's efforts to incorporate her expectations for teachers into her own practices as principal, especially her belief that educators have to take full responsibility for student success:

I really see her modeling for us. She's discreet about that. She doesn't stand up and say, "I'm going to do these things, you watch me do it." ... She's very available for students, whether it's something formal like being able to have lunch with the principal for being a good citizen or whether it's some kids who actually need help with some learning task and they're in her office trying to finish a project they didn't get done because they didn't have the support they needed at home. You see her doing those kinds of things. You see her when the lunch line is backed up with the Thanksgiving lunch, and you see her back there with an apron on putting gravy on people's potatoes. There are not jobs here that she is not willing to do.... Y You teach by example. (DB, 11/20)

Effects on Teachers. Teachers at Cane Ridge shared a sense of personal responsibility for student success. "We have a role here, and we're here for a reason," Mr. Baker reported. "In terms of meeting the needs of those children, when you get around to teaching and learning that's what it's all about" (DB, 11/20).

Ms. Hammond described the teaching staff as embracing a "no excuses" attitude:

We can't make excuses [such as] "This child might come from a bad home life." We've got several children who have problems. Who doesn't anymore? Even though you can't use that as an excuse, it certainly can be a cause. So is there anything we can do on that level? Even though it can't be an excuse, does that mean we can blow them off? No, they have a bad home situation. Now what are we going to do about it? (DH, 10/16) 
Among the things the teachers at Cane Ridge "do about it" were efforts to review student achievement data to modify instruction, to connect students with counseling or family support when necessary, and to work together to develop interventions to help students who are struggling (DH, 10/16; ME, 9/25). Edmonds added, "They wear my email out and so much of it is a specific concern about a specific child" (ME, 9/25).

Part of the way teachers take responsibility, according to Hammond, was by being self-reflective about the worth of various instructional activities.

We're always asking ourselves, this sounds like a neat activity or cool thing to do with the kids, but is it worthwhile? Is it going to meet what I need to do? Is it going to work toward the goals that we've set here? It's not just a cute little unit that I've pulled off the internet because it sounded like fun. (DH, 10/16)

Finally, the sense of personal responsibility for student outcomes extended to a strong desire on the part of the teachers to meet Ms. Edmonds's expectations.

According to Hammond, there was sometimes an added layer of stress because of this internal desire to please the principal, but "stress can be a good thing. It keeps us accountable." Edmonds did not intentionally put pressure on teachers to satisfy her expectations, but the teachers internalized that desire anyway:

As one teacher said, "It's hard to be a teacher here because you can't just come in at 8:00 and leave at 3:30 and say, 'My job is done." Because even though Ms. Edmonds isn't going to be on your back constantly going, "Are you using this? Are you doing this?" you know what her standards are and you respect her because of that, so you want to live up to those standards. You are constantly asking yourself, "Am I doing all I can? Am I doing what Ms. Edmonds wants?" (DH, 10/16)

Primarily, the teachers reported a desire to meet Edmonds's expectations because they admired and respected her leadership and her vision for the school. Ms. Jones explained, "Because I see her as such a leader that believes in what she's doing and wants the best for the children, it makes me want the best for the children myself" (JJ, 10/16). 
Edmonds's long working hours were also a source of motivation and inspiration for the teachers. "Sometimes I feel guilty because she does so many things," Jones shared. "You call here at 7:30, 8:00 at night, you've got a question, and chances are she's going to pick up the phone when it rings," Mr. Baker reported. "It's hard not to have a great deal of respect for a person like that. You don't want to disappoint her" (DB, 11/20). Baker believed that teachers should try to inspire their students through positive personal relationships in the same way Edmonds inspired and motivated the teachers:

It does come back to relationship.... I don't want her to be disappointed. I want to meet the expectations that I understand she has for me. If we could carry that on over to our classroom and have relationships with these kids, such that they wanted to do what we ask them to do, because they want to better themselves, but also because they actually have some consideration for what we hope to see out of them, what we recognize as their best effort—I think that's important. (DB, $11 / 20)$

In summary, Edmonds assumed that focusing on the needs of individual students could lead to higher student achievement. This assumption shaped a theory of practice that featured action strategies including consistently communicating a vision that all children could learn and that educators are responsible for that learning, organizing school activities to celebrate student diversity and student success, and modeling hard work and positive relationships with students. Teachers reported that this theory of action affected them by encouraging a stronger sense of responsibility for student success, higher levels of motivation, and a desire to meet the principal's expectations. Theory of Practice 2: Nurturing Positive Personal Relationships with Teachers

Based on the assumption that positive personal relationships motivated teachers as well as students to higher levels of performance, Ms. Edmonds actively supported teachers on a personal level, responding to a wide variety of their needs. Teachers in turn 
reported strong appreciation for the principal, and high levels of job satisfaction (see

Figure 8).

ASSUMPTIONS

The principal's role as
both a human and a
manager is to affirm and
value teachers as
human beings

2

When teachers feel affirmed and valued as human beings, they will be more motivated and effective as professionals

1 All people possess inherent worth and value and deserve to be treated with dignity and respect
ACTION STRATEGIES

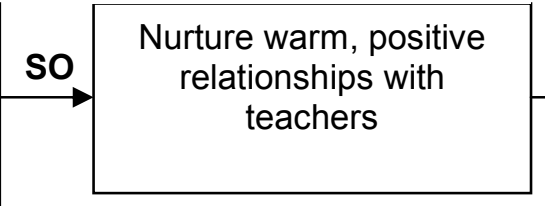

1. Be available to listen, answer questions and relate to staff on a human level

2. Give corrective feedback in a positive, caring way

3. Be supportive of teachers in times of personal crisis and loss

4. Offer praise and appreciation for teachers' efforts and achievements

\section{TEACHER EFFECTS}

Teachers feel valued and affirmed, satisfied with their jobs and work environment

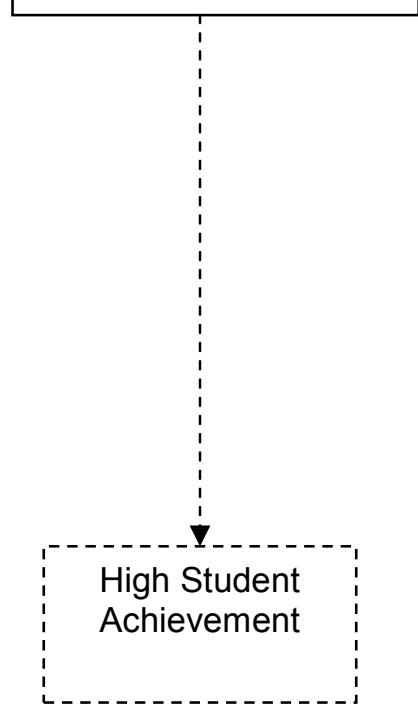

Figure 8. Marie Edmonds, Theory of Practice 2, for nurturing positive relationships with teachers, including assumptions, action strategies and teacher effects. A tacit assumption of all instructional leadership theories of practice is that these action strategies will indirectly result in higher student achievement, though this link is not explored in this study (indicated by dashed lines in the figure).

Assumptions. Edmonds assumed that teachers who were affirmed and valued

would experience greater levels of self-efficacy and satisfaction:

Just as students have higher achievement when they have positive relationships with their teachers, I believe that teachers are more motivated to work harder to meet student needs when they know what they do is appreciated and supported by their administrator. I want all our staff to enjoy coming to work each day because they enjoy working together, planning together, and problem solving together. We are like a family that celebrates the successes of each of its members and bears each other's burdens when things are not going well personally or professionally. (ME, 3/10) 
Teachers at Cane Ridge insisted that Ms. Edmonds's attention to the personal needs of others was a part of her personality, and not just a management strategy. "I think it is just a part of who she is as a Christian woman," Ms. Hammond related. "She values people on a personal level. It wouldn't matter if she were a principal, office employee or stay-at-home mom, she cares about others. I feel this is one reason it works for her. There is no planning or forethought in her actions, it comes naturally" $(\mathrm{DH}, 3 / 9)$.

Mr. Baxter agreed, linking Ms. Edmonds's assumptions about how to treat others to the principal's religious faith:

Mrs. Edmonds is never, ever preachy. But she teaches so much by her example. The fairness with which she treats everyone is so much more than a management strategy. It is her commitment to treating others - students, parents, faculty and staff-as she would wish to be treated herself. This is the type of person Ms. Edmonds would be, regardless of the career path she has chosen. (DB, 3/10)

Action Strategies. Edmonds believed that she had an important responsibility to be available to her staff in a nurturing, positive way, both for their professional and personal needs:

[I want to be accessible] if they have questions, concerns, need to vent, need to celebrate. ... Part of my role as instructional leader is to make time for those kinds of things. ... I don't want any teachers to ever feel like they have questions, concerns, problems that they are alone and there's nobody to turn to for help. (ME, 9/25)

She framed her action strategies in terms of being available for teachers to share their needs. "I really spend a lot of time listening and a lot of time asking questions. I describe my leadership style as 'management by walking around.' Some leadership guru came up with that, but if I stayed in my office all day and sat at my computer I wouldn't have a clue, so I'm out in the building" (ME, 9/25). 
Jones, Hammond, and Baker all confirmed that this was an important part of Edmonds's instructional leadership, and formed the basis of one of her core action strategies. Mr. Baker, for example, reported, “I don't know that there's ever been a time that I have needed to speak to her [that she didn't make herself available]. . . Even if it's just stopping by her office and she's working on the computer, she turns around and her focus is on you. So you begin to feel kind of important and wanted and needed" (DB, $11 / 20$.

According to Ms. Jones, "She's able to build you up, even when there's a problem." She described an incident when Ms. Edmonds had to approach her with a problem regarding Jones's own child, who was a student at the school. "It was a very difficult thing, but she did it with such grace and tact, and I did not feel threatened. She just has a way about her that is serious, but not threatening, and she's willing to talk with us and help us" (JJ, 10/16). Jones gave other examples of how Edmonds had been personally supportive of her in times of need:

When there's a crisis, she'll sometimes call the faculty to work if we want to come early and pray. If there's a situation, she's the first one to be there. She always visits everybody in the hospital. If there's a death, she's right there. Both my parents have died while I've been working here. Gosh, she was so kind and supportive. With my father I was out for an extended leave, just because there were a lot of things I had to take care of being an only child, and she didn't in any way reprimand. She was so understanding about that. (JJ, 10/16)

Ms. Hammond pointed out Edmonds's practice of sending notes to teachers to praise them for accomplishments and their effort to help students: "She sends notes, not just to the teacher, but to your spouse thanking them for the time they allow their spouse to be at school. That means a lot to the spouse, but it also means a lot to you because she recognizes that I'm giving up time with my family to do my job" (DH, 10/16). 
Baker also noted Edmonds's commitment to do a formal classroom observation with every teacher each year (even those who were tenured and were no longer required to be observed yearly). Her approach to these observations was always supportive and positive. 'It's not at all a threatening thing. You don't get scared because Ms. Edmonds is going to come watch you teach" (DB, 11/20).

Effects on Teachers. Asked about the effects of her instructional leadership, Edmonds did not mention test scores but spoke passionately about the environment at Cane Ridge. "We have happy kids and happy teachers here. I would describe it as a positive, warm school culture. We have an appreciation and a celebration of differences" (ME, 9/25).

Ms. Jones agreed with Edmonds's assessment of the school culture and credited her leadership as its source.: "[The school is] like a family. We have spats, but she wants us to work through that. We're here for the kids and keep that in mind. It's warm and fuzzy." Ms. Jones worked with eleven principals over the course of her career and Ms. Edmonds "is by far my favorite. I feel happy [here]. If I ever had to leave this school I'd be very upset" (JJ, 10/16).

Ms. Hammond attributed her success as curriculum specialist for the last eleven years to Edmonds's support. "I would not have been able to stay in this positionbecause it's a stressful spot — as long as I have if I hadn't had a principal I could work with" (DH, 10/16).

"I can't really imagine having a better working situation,” Mr. Baker related. "I feel valued here." Baker believed the rest of the faculty shared his sentiments:

We really are happy to be here, thankful for working in this situation because we know full well that not all schools operate the same way. We've got enough 
friends teaching other places that some of these things I'm speaking about as far as values and behaviors and respect - that's not across the board every place you go. (DB, 11/20)

To summarize Theory of Practice 2, Edmonds assumed that when teachers were valued and affirmed in their work, their levels of job motivation and efficacy would increase. Teachers described this assumption as a key part of Edmonds's moral code, rather than a mere management strategy. Edmonds genuinely valued the teachers as human beings and cared about them as people. As a result, she engaged in action strategies such as making herself available to teachers who needed advice and encouragement, offering support to teachers in times of personal crisis, and giving praise and positive feedback. According to teachers, this theory of practice caused them to feel appreciated and affirmed as individuals, and increased their sense of identity and loyalty to the school.

\section{Theory of Practice 3: Continual Professional Learning}

Ms. Edmonds believed that the challenges of educating today's children were so great that educators must engage in on-going professional growth and development, and that the most effective forms of professional learning involved collaboration and sharing between teachers and with the principal. As a result, Edmonds actively fostered collaboration and idea sharing among teachers and modeled on-going professional growth by herself engaging in reading, research and conference-going and sharing new ideas with others. As a result, teachers at Cane Ridge engaged in a high degree of collaboration and respected Edmonds's authority as an instructional leader (see Figure 9).

Assumptions. Ms. Edmonds described the commitment to teacher collaboration as one of her "deep-seated" assumptions. 
ASSUMPTIONS

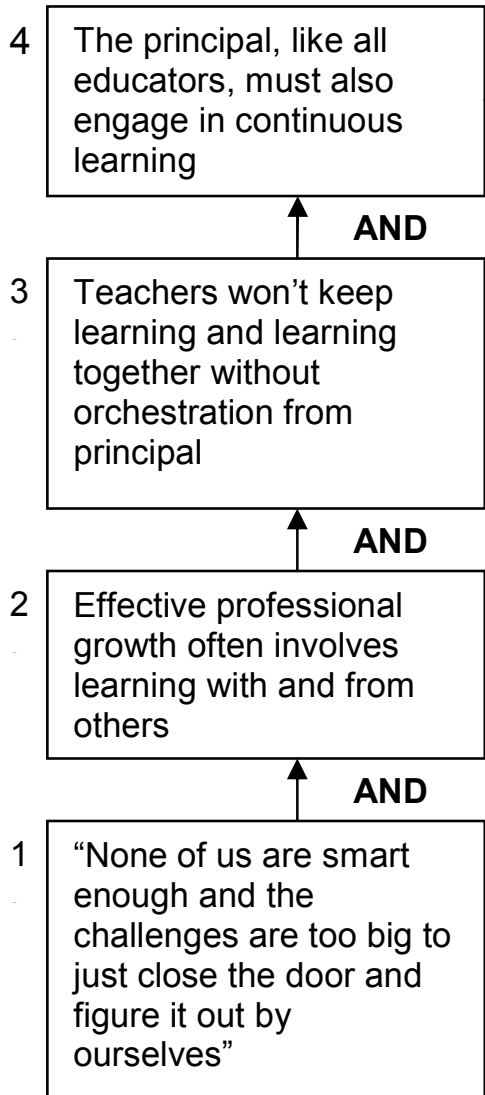

ACTION STRATEGIES

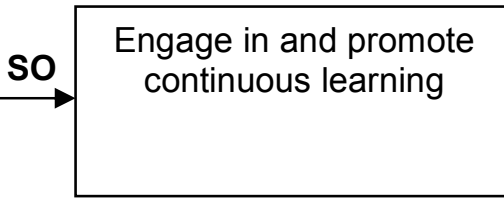

Examples:

1. Model ongoing learning by attending conferences and reading research

2. Share new learning with staff

3. Promote opportunities for teacher learning and professional development, especially through collaboration
TEACHER EFFECTS

Teachers respect Ms. Edmonds's ideas because they know her ideas are well grounded and they feel responsible for implementing her suggestions; teachers collaborate and learn from each other

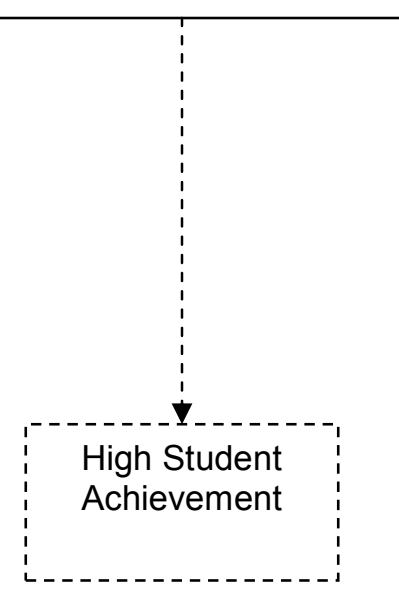

Figure 9. Marie Edmonds, Theory of Practice 3, for encouraging continuous professional learning, including assumptions, action strategies and teacher effects. A tacit assumption of all instructional leadership theories of practice is that these action strategies will indirectly result in higher student achievement, though this link is not explored in this study (indicated by dashed lines in the figure).

Teachers teach best when they are a part of a professional learning community and are always learning and supporting each other and free to be risk takers and to just lay it out there.... None of us is smart enough and the challenges are too big to just close the door and figure it out by ourselves. (ME, 9/25)

According to Mr. Baker, Edmonds's support for collaboration was rooted in her

confidence in teacher abilities to solve problems as professionals:

She is not a dictator at all. She certainly has expectations for us, but it's seldom that she just tells us what we have to do. There may be a particular goal that we need to reach, but she's very respectful of our input as we figure out how to reach the particular goal. ... I feel that she is very much a consensus builder. (DB, $11 / 20)$ 
Action Strategies. Based on her assumption that teachers should function as a collaborative, professional learning community, Ms. Edmonds engaged in intentional efforts to encourage teachers to learn from and support one another. She made copious notes of activities she observed in classrooms or student work displayed in the hallways and then recognized those teachers in staff meetings or through e-mail messages: "It's a brag for those teachers," she shared, "but then, I'm thinking it will kind of encourage others to try some of these strategies, and if they are uncertain about it, hopefully it will open the door for them to ask [the other teacher] about that strategy" (ME, 9/25).

Edmonds noted examples of her efforts to partner experienced, National Board certified teachers with less experienced teachers. A kindergarten teacher with experience at the fourth and sixth grade levels was mentoring a sixth grade teacher in an early-morning reading enrichment project for struggling students. "They are having the best time," Edmonds reported. "I was praising them this morning for giving up the time and really putting forth the effort, and they said, 'We're loving it.' It's those kinds of ways you can connect teachers together" (ME, 9/25).

The teachers confirmed this support of collaboration as one of Edmonds's main instructional leadership action strategies, especially the tactic of partnering experienced and inexperienced teachers. Ms. Jones noted Edmonds's efforts to bring in a retired teacher two to three days a week to help one struggling teacher (JJ, 10/16). Mr. Baker also pointed to Edmonds's policy of including a team of teachers for all interview and hiring decisions and the existence of a behavior support committee to review, refine and develop discipline policy as other examples (DB, 11/20). 
The researcher observed numerous examples of Ms. Edmonds fostering teacher collaboration and of teachers working collaboratively. During one faculty meeting, teachers facilitated a training session on a new technique for teaching the writing process, and while Edmonds introduced the topic, she simply participated in the learning alongside the teachers (Observation, 11/7). At another faculty meeting, she facilitated a workshop in which teachers worked in small groups to analyze CATS test data (Observation, 10/24). On another occasion, teachers working on the Reading First grant implementation team met in Edmonds's office while she went about her other duties, only stopping in at the end of the meeting for a report (Observation, 11/7).

Edmonds prided herself on being a life-long learner and believed that she must continue to learn and study to improve her craft as principal and improve the success of her school:

My role is to take very seriously the development of these children academically as well as socially and to educate myself as to what are the best practices in reading, mathematics, science and social studies so that I can support the teachers who are doing the right things and I can help the teachers who are struggling a bit. (ME, 9/25)

She attended conferences and professional development seminars, and participated in the same training her teachers received, and the interviewed teachers confirmed this $(\mathrm{DH}$, 10/16). Edmonds also participated in professional organizations and served as a state officer in the Kentucky Association of Elementary School Principals. At least six tote bags from various conferences she had attended were on the floor of her office (Observation, 10/31).

Effects on Teachers. The teachers at Cane Ridge responded to Edmonds's assumptions and behaviors regarding collaboration by eagerly and actively engaging in 
sharing and collaborative decision-making. Edmonds considered it a hallmark of the school's culture:

If fact, some of our teachers that left and went to other schools are struggling a bit because they [their new schools] are not quite as collaborative. That's kind of the culture here. In fact, one of them was offered a job in another school and has come back just because she said, "I have to team. I don't want to learn how to teach by myself." (ME, 9/25)

Examples of teacher collaboration observed or documented in this study included teacher participation in the interviewing and hiring process (ME, 9/25; DB, 11/20), veteran and new teachers partnering on teaching projects (ME, 9/25; JJ, 10/16), retired teachers assisting struggling teachers (JJ, 10/16), self-directed teacher teams implementing the federal Reading First grant (Observation, 11/7), teacher-led professional development (Observation, 11/7) and Edmonds's cooperation with the curriculum specialist to implement new instructional initiatives (DH, 10/16).

According to Ms. Hammond, the school's curriculum coordinator, the staff respected Edmonds's instructional knowledge because they knew she thoughtfully engaged in ongoing learning and professional growth herself:

I have worked for principals who propose something and you go, 'Yeah, that'll be gone in two weeks.' Because they heard it at a conference, they come back and tell you about it, but then you never hear any more about it. You know that Ms. Edmonds has already sifted through and pulled out what is best for us, so she's serious about it. $(\mathrm{DH}, 10 / 16)$

Hammond saw the staff moving toward ever-deeper levels of collaboration:

We've worked really hard on it this year, too, as a conscious decision, to walk in with a blank agenda with our leadership teams and say, "What would you like to see happen?" instead of, "This is what we need to do this year." Give them more decision making in where we need to go. (DH, 10/16)

Edmonds found that teachers were so accustomed to collaboration, they sometimes challenged her on routine decisions that she expected to make on her own: 
Sometimes I think, "Can I not make a single decision myself?" Because it is such a collaborative environment, that if I do make a decision without consulting with several people, they are like, "She didn't ask me about that. Why didn't we talk about that?" (ME, 9/25)

Edmonds's third theory of practice was based on an assumption that educators must engage in continual professional learning to ensure high levels of student achievement, and that teachers learned best when they engaged in on-going collaboration with one another. Her action strategies included encouraging teacher sharing, partnering experienced and novice teachers, and modeling continual learning by participating in professional organizations, attending conferences, and reading research literature. Teachers reported high levels of collaboration as a result of Edmonds's theory of practice, and respected her as an instructional leader because she had proven herself an authority by herself modeling life-long learning.

\section{Theory of Practice 4: Inviting Teacher Input}

Ms. Edmonds made intentional efforts to invite teacher input into decisionmaking because she assumed that if teachers had ownership for the overall direction of the school they would have ownership over student achievement outcomes. She did this through establishing faculty committees, engaging in formal and informal discussions with the staff, and actively soliciting teacher feedback. As a result, teachers were empowered and entitled to a strong role in school governance. Edmonds and teacher participants generally viewed this as a positive outcome, but noted that at times teacher expectation for input was challenging because it over-lapped into Edmonds's responsibility as administrator to have the final say. She described her efforts to become more intentional and careful about soliciting teacher input over the years (see Figure 10). 
ASSUMPTIONS

\begin{tabular}{|c|c|}
\hline+ & $\begin{array}{l}\text { The principal, like all } \\
\text { educators, must also } \\
\text { engage in continuous } \\
\text { learning }\end{array}$ \\
\hline & AND \\
\hline \multirow[t]{2}{*}{3} & $\begin{array}{l}\text { Teachers won't keep } \\
\text { learning and learning } \\
\text { together without } \\
\text { orchestration from } \\
\text { principal }\end{array}$ \\
\hline & so \\
\hline \multirow[t]{2}{*}{2} & $\begin{array}{l}\text { Effective professional } \\
\text { growth often involves } \\
\text { learning with and from } \\
\text { others }\end{array}$ \\
\hline & so \\
\hline 1 & $\begin{array}{l}\text { "None of us are smart } \\
\text { enough and the } \\
\text { challenges are too big to } \\
\text { just close the door and } \\
\text { figure it out by } \\
\text { ourselves" }\end{array}$ \\
\hline
\end{tabular}

ACTION STRATEGIES

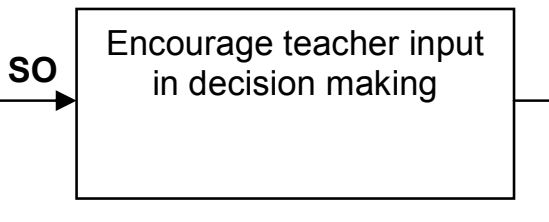

Examples:

1. Teacher committees

2. Teacher involvement in hiring process

3. Soliciting teacher feedback

\section{TEACHER EFFECTS}

Teachers participate in decision making

Figure 10. Marie Edmonds, Theory of Practice 4, for inviting teacher input, including assumptions, action strategies and teacher effects. A tacit assumption of all instructional leadership theories of practice is that these action strategies will indirectly result in higher student achievement, though this link is not explored in this study (indicated by dashed lines in the figure).

Assumptions. Just as Edmonds believed that the challenges of educating today's

students were too big for a single teacher to have all the answers, she also assumed that as principal she needed the input and ideas of others to be most effective. "She has said many times that she does not want to make all the decisions," Ms. Hammond explained. "She values teachers who have experience in various areas. She realizes that she cannot know all, experience all, read all about a particular subject or topic. She has some wonderful resources in her building and she is not afraid to use them" (DH, 3/8). 
Edmonds understood inviting teacher input to be a component of nurturing a collaborative environment, and since much of the knowledge about learning issues arose from teacher interactions with students, teachers were in the best position to contribute to solutions:

When a teacher identifies a problem, I ask how he or she would like to see it solved. ... It really is a philosophy that teacher input is welcome no matter what the issue. Teachers feel very comfortable to give input on just about everything by e-mail, written note, or in person. I believe teacher input is important because we are all working together for the good of the students and the good of the school and having input builds ownership. We all own our problems and we all own our successes. Teacher input has a very positive effect on our school. We have creative ideas and solutions flowing all the time. (ME, 3/10)

Action Strategies. Ms. Edmonds used a variety of strategies for inviting teacher input, including encouraging faculty committees, informally discussing problems with teachers, and actively soliciting teacher feedback during faculty meetings and using surveys (DH, 3/8; DB, 3/10; JJ, 3/11). Ms. Hammond described how Edmonds used an interactive computer program to conduct instant surveys with teachers at a faculty meeting earlier in the year to gauge teacher perceptions of the school climate and, on another occasion, to assess teacher comfort level with instruction for gifted students (DH, 3/9).

"She wants our input," said Ms. Jones. "It's almost like a government. She wants everybody to be part of this democracy" (JJ, 10/16). Edmonds sometimes provided teachers lists of issues and asked teachers to rate them in terms of their perceived order of importance, and asked teachers to complete online surveys related to student behavioral issues (JJ, 3/11).

Baker described how Edmonds invited him to attend a number of informational meetings with her on topics related to special education interventions and the impact of 
the state's inclusion of the American College Test (ACT) in high school accountability on elementary education:

Ms. Edmonds's philosophy is that the rest of our faculty is more likely to buy in to ideas that already have some support among their colleagues, rather than everything coming down as a "directive" from the top. Quite often, she relies upon the leadership of her faculty to share new ideas in small groups rather than dropping some bombshell in a faculty meeting.... The bottom line is that she does not do much micro managing. She hires people she believes she can trust, and then she expects all of us in one way or another to take on an active leadership role. ... She empowers people. (DB, 3/10)

Effects on Teachers. Baker, Jones, and Hammond reported high levels of empowerment and valued their involvement in school-wide decision-making. "This is something she nurtures and cultivates and it makes us all feel valued" (DB, 3/10). "We have a stake in how this building runs and how we are viewed in the community" (JJ, 3/11). "Teachers feel important, that what they are doing and what they know is important," Hammond said. "Our teachers greatly admire Ms. Edmonds and to have her value their opinion is a treasure. She gets buy-in/ownership when she values teachers" (DH, 3/8).

Inviting input was "most definitely" a hallmark of Edmonds's leadership, according to Hammond, "to a fault":

She is so open to teacher input that sometimes our teachers forget that the ultimate decision is hers. She is our instructional leader and the buck stops with her. Yet, some teachers feel they have been "pushed aside" when she does not follow what they have suggested. What they don't realize is that there are many factors that go into a decision. Our teachers do not feel as if she is a dictator and they are merely the servants fulfilling her wishes. This is not the case at all. Because she seeks input from others, they feel that ownership. (DH, 3/8)

In summary, Edmonds assumed the task of raising student achievement was so complex, it required the contributions and input of all stakeholders. She relied on the wisdom, experience and insight of teachers to inform her decision making as principal. 
Based on these assumptions, Edmonds invited faculty input through establishing committees, engaging in formal and informal discussions of important topics, and soliciting teacher feedback. As a result, teachers believed Edmonds's genuinely valued their opinions and were empowered to participate in school-wide decision making. Theory of Practice 5: Utilizing Directive Leadership when Necessary

Teachers and Ms. Edmonds herself were all consistent in describing her effort to become more directive as one of the key changes in Edmond's leadership over the years. That assertiveness sometimes clashed with the collaborative environment of the school. This phenomenon seemed to manifest as a "special-case" theory of practice. When Edmonds's normal practice of using collaboration, modeling and using positive personal relationships proved ineffective, she utilized more directive forms of leadership such as corrective feedback and giving specific orders (see Figure 11). Edmonds acknowledged that these strategies were outside her comfort zone, but were necessary in cases where student achievement or the overall mission of the school were at stake.

Assumptions. Edmonds related her reluctance to be more directive to her basic assumption that to be effective as an instructional leader, she should rely primarily on positive personal relationships with teachers and to adjust her management style to their individual needs. Edmonds acknowledged that despite this warm, personal approach, sometimes teachers still failed to commit to her core vision of what the school should become, and a more directive approach became necessary. Even for those who needed more direction, her personal relationships with staff became the foundation for knowing their needs:

I think we have to know our teachers very well, because the way we respond, I could have the same scenario with someone else, and somebody being directive 
ASSUMPTIONS

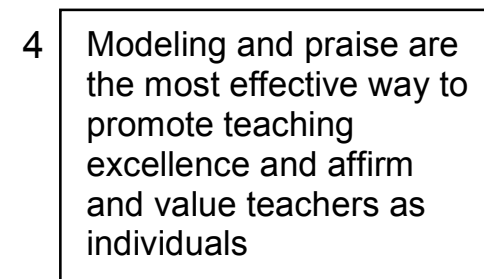

3

The principal's role as both a human and a manager is to affirm and value teachers as human beings

\begin{tabular}{l}
2 \begin{tabular}{l|}
\hline \multicolumn{1}{|c|}{ AND } \\
affirmed and valued as \\
human beings, they will be \\
more motivated and \\
effective as \\
professionals
\end{tabular} \\
$1 \begin{array}{l}\text { All people possess } \\
\text { inherent worth and value } \\
\text { and deserve to be } \\
\text { treated with dignity and } \\
\text { respect }\end{array}$ \\
\hline
\end{tabular}

ACTION STRATEGIES

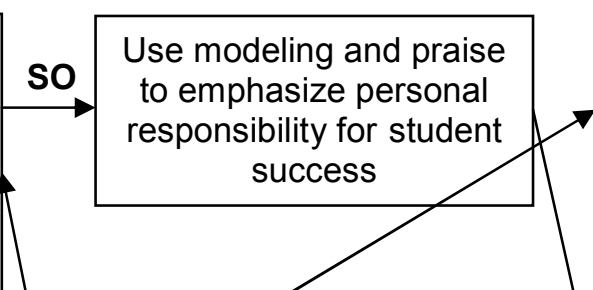

Revised Action Strategy:

Use directive feedback to help teachers feel responsible for student success

\section{TEACHER EFFECTS}

Teachers are responsible for meeting individual student needs; want to meet the principal's expectations

Some teachers do not respond to modeling and praise

Figure 11. Marie Edmonds, Theory of Practice 5, for using directive leadership, including assumptions, action strategies and teacher effects. This is a "special case" theory of practice that reflects "double-loop" learning. A tacit assumption of all instructional leadership theories of practice is that these action strategies will indirectly result in higher student achievement, though this link is not explored in this study (indicated by dashed lines in the figure).

might be the best approach, and somebody else it might just discourage them and make them feel like they're not doing anything right. My natural approach is to accentuate the positive, encourage, invite them, facilitate them visiting someone else's class who is doing it the way I like to see it done. (ME, 12/4)

Edmonds recognized that her responsibility in communicating a vision for

learning in the school drove her to be more directive, but that typically this was

overshadowed by her commitment to a collaborative culture: "I agree that the 
collaboration component trumps the communication component as long as my underlying values [about the mission of the school] remain intact" (ME, 12/4).

Action Strategies. Ms. Edmonds was directive in giving specific, corrective feedback to teachers when necessary or in giving assertive orders. Teachers at Cane Ridge reported that these behaviors were rare for Edmonds, but that she had grown to utilize them more over the years.

"She's had to get tougher," Ms. Hammond shared:

It's not in her to be the tough leader and to have to go to people and say, "No, you've got to do it like this." I think she's seeing that some people don't get it just by example or by whole group instruction. Or maybe they don't understand quite how to implement it, so she's had to become a little harder, a little more personal, one-on-one, with, "You need to do this." She'd much rather lead by example, by modeling than by being that tough. Different people have different personalities and some people just don't get it that way. (DH, 10/16)

Ms. Jones agreed. "I see her as being more firm," especially concerning parents.

Jones acknowledged that this action strategy did not come easily to Edmonds:

She'll call a parent in a heartbeat [now] whereas she might have waited a little while before. Although she did tell me one time, "If a parent calls you and they are really, really upset, don't call them immediately. Wait a few hours, but don't delay too long because then they're going to think you are avoiding them." There's a window. She probably waits until the end of that window and then calls, but she's pretty good about resolving [parent] issues. I just see her as firmer. (JJ, 10/16)

Edmonds agreed that she had become more directive over the years, but also agreed that it was a struggle for her and that it came with a price. In the reflective exercise in which she recounted a post-observation conference of a lesson that did not go well, Edmonds described a teacher who taught a lesson on a short story but did not teach the vocabulary necessary for the students to understand the story and be engaged. In her recollection of the conference, her conversation with the teacher focused on whether the 
students actually knew the vocabulary or not, and the teacher's explanations for why the students were not engaged:

P (Principal): What went well in the lesson?

T: (Teacher): The children liked the story.

P: Yes, it is a great story-lots of descriptive language and rich vocabulary. The vocabulary was key to the story. Do you think the children knew what a saddle, bridle, and stirrups were?

T: Of course they do. They watch TV. They can see it in the pictures.

P: What could you do to build background vocabulary for the story?

T: There just isn't time to do everything we'd like to do like bring in a saddle or show lots of pictures. Then the children get to talking and before you know it, there's no time for reading.

P: Building vocabulary is essential for reading success. That time spent at the front of the lesson builds interest and understanding. I am concerned about the number of students who weren't engaged in the lesson- "restless," as you called it.

T: Oh, you know. Billy and Joey are such problems. They are from bad homes. (ME, 12/4)

In the column in which she related the thoughts and feelings she was experiencing during the post-observation conference, Edmonds described her frustration regarding this teacher, and her doubts that any amount of directive management would improve her teaching. "She's making excuses," she wrote. "She's missing the point. How do I turn this negativity around? Is it possible for her to change?" (ME, 12/4). She also wondered, in retrospect, whether she should have been more directive or more positive: "Should I have been more directive? Should I have just focused on what was going well?" (ME, 12/4).

She did eventually become more directive with the teacher, but her efforts did not improve the teacher's performance. Edmonds explained that the teacher eventually left the school after an extended medical leave.

Effect on Teachers. Ms. Edmonds's occasional use of directive leadership, while difficult for her, was effective in gaining teacher compliance. "It is very hard for me, and 
I think because it's not my style, it's pretty shocking to people when I say, 'This is what you need to do, this is the way you need to do it now, and I will be checking.' But occasionally that's effective because I don't do it very often" (ME, 12/4).

On those rare occasions when she became directive with teachers, Ms. Hammond described the dramatic response. "They'll come to me and say [feigning upset], "She's disappointed in me!" I have lots of boxes of Kleenex. I'll say, "No, it's not that." Because she does this so seldom that when she does, it really does affect people" (DH, 10/16).

When she was directive, Edmonds often experienced a backlash from teachers who had come to rely on her collaborative approach. In interviews, she related a recent incident in which she planned a parent informational meeting regarding a school-wide reading assessment without consulting teachers. The teachers were reluctant to share individual student data with parents in an unstructured forum. Parent-teacher conferences were scheduled for just two weeks later, and the teachers preferred to give individual student information out in that one-on-one, pre-scheduled format. When the teachers protested, Edmonds called a special meeting to discuss the matter, and collectively she and the faculty decided to proceed with the meeting, but only share generic information about the assessment and overall student achievement, and then share individual student data at parent-teacher conferences (ME, 9/25; 12/4). Ms. Edmonds expressed some frustration over the incident ("Can I not make a single decision myself?" she asked), but said she had learned that her school culture required her to take a more collaborative approach:

Why didn't I poll a little group of teachers before I sent out a blanket e-mail [saying], "This is what we are going to do?" After all these years, you'd think I 
would anticipate something like that. I guess I've been out of the classroom long enough that the red flags that set off in their mind didn't set those off in my mind. (ME, 12/4)

Her comments suggested that Ms. Edmonds' theories of practice for inviting teacher input and collaboration sometimes compete with her responsibilities to be the final authority and protect the core mission of the school. She exhibited a clear reluctance to use directive leadership, and resorted to that theory of practice only when she believed no other theory of practice would be effective. In this sense, Edmonds's fifth theory of practice, based on the assumption that sometimes teachers need corrective, directive feedback to improve their performance, may constitute a form of double-loop learning. When her preferred theory of practice (using modeling and positive feedback) failed to achieve its intended outcome (teacher effectiveness), she revised her assumptions and developed a "special case" theory of practice to account for teachers who needed a different approach to improve their performance.

In summary, for Research Question 1, data from Case Study A revealed that Marie Edmonds used five theories of practice that accounted for her instructional leadership behaviors, including the following: (a) modeling and communicating a vision of schooling that focused on meeting the needs of the individual child, (b) nurturing positive personal relationships with teachers, (c) emphasizing continual professional learning, primarily through collaboration, (d) inviting teacher input into decision making, and (e) using directive forms of feedback when modeling and praise did not work to ensure teacher effectiveness.

For Research Question 2, data indicated that teachers responded to Edmonds's leadership in the following ways: (a) teachers had a sense of responsibility for student 
outcomes and desired to meet the principal's expectations, (b) teachers felt valued and appreciated in their work, and reported high levels of motivation and job satisfaction, (c) teachers engaged in teamwork and collaboration, and (d) teachers were empowered to participate in school-wide decision-making processes.

Finally, for Research Question 3, Marie Edmonds demonstrated reflection-inaction in that during her years as principal she had learned the importance of giving directive, corrective feedback when her preferred methods of modeling and using praise to encourage teacher effectiveness failed to work. Teachers confirmed this had been a growth area for Edmonds, and data suggested that Edmonds developed a "special case" theory of practice to account for situations when her normal leadership strategies did not work to encourage teacher improvement efforts.

\section{Case Study B}

\section{Donna Rippy: Leading from the Heart}

Donna Rippy, principal of Cherrywood Elementary School, was a 31-year veteran of education. Rippy taught high school business courses for 13 years prior to becoming a high school guidance counselor, a position she held for 11 years before briefly serving as an assistant principal. Seven years ago, she entered her first principalship at Cherrywood Elementary, a brand new school built in a fast-growing district of 12,000 students in South Central Kentucky. Cherrywood served students from the same area of the county as the high school where she served as guidance counselor and assistant principal. Rippy's theories of practice of instructional leadership were rooted in strongly-held assumptions about the inherent potential of every child to be successful and the critical role of strong, caring relationships in student achievement. 


\section{School Context}

Cherrywood Elementary served 743 students in pre-school through sixth grades. The school retained the fresh, polished look of a new building and sat next door to the district's central office near a large community soccer complex and residential neighborhood. Cherrywood's staff of 38 certified teachers served students from both affluent suburbs (including one neighborhood of million-dollar homes) and from the area's public housing authority. The student population included $36 \%$ on free or reduced lunch, $18 \%$ minority students, and $9 \%$ special education. Like the larger community, CES's student population had become more diverse with a large influx of immigrant families, including a recent group of Burmese children. The school identified approximately $4 \%$ of the students as English language learners (ELL).

Despite the challenges posed by an increasingly diverse student population, Cherrywood Elementary posted strong student test scores from its inception, and scores continued to rise rapidly as measured by the Commonwealth Accountability Testing System (CATS), Kentucky's statewide student achievement program. By state policy, all schools must achieve an academic index of 100, indicating that almost all students are at proficient levels of performance in core subject areas, by 2014. The state measures schools' progress in two-year increments and Cherrywood's academic index rose from 77.7 in the biennium ending in 2002 to 90.4 in 2004 and 96.8 in 2006 . The 2007 index was 107.7, the highest of any school in the district, but that score will be averaged with the 2008 results to calculate an official biennium score. Rippy credited the quality of her teaching staff, a collaborative professional atmosphere, and above all the positive, student-friendly environment, for the school's success. She also believed divine 
providence played a role, and downplayed her own part in the student outcomes: "I think it's a God thing. I don't think it's a Donna thing. . . . If you could have told me [when the school opened] what we have right now was the way it was going to happen, I wouldn't have believed you. It's been a blessing” (DR, 1/22).

A "family-oriented environment" was the hallmark of the school (DR, 1/22). The motto, "Life is good at Cherrywood," appeared on a large bulletin board in the main office and on the t-shirts worn by cafeteria staff (Observation, 1/22; 2/1). Teacher participants in the study quickly identified the positive school climate as a key to understanding Cherrywood's success (DH, 1/25; JW, 1/30; SY, 2/1). Rippy's theories of practice were strongly driven by a desire to create a positive learning climate that built capacity for student and teacher success. Data indicated her leadership fostered just such a community at Cherrywood Elementary.

\section{Donna Rippy: Research Findings}

Six theories of practice described Ms. Rippy’s instructional leadership. Each theory of practice is described below, including the core assumptions that made up the foundation of the theory of practice, the action strategies that logically emerged from those assumptions (Research Question 1), and the effects of the theory of practice on teachers (Research Question 2). When data suggested that Rippy had engaged in reflective practice by adjusting or modifying her theories of practice by revising her assumptions or action strategies, this process is also described (Research Question 3).

\section{Theory of Practice 1: Nurturing a Positive Learning Climate}

The dominant feature of Rippy's leadership was an emphasis on positive relationships, exemplified in the idea that a school community should function like a 
"family" (DR, 1/22). Rippy assumed positive relationships among teachers and students leads to academic success and that her responsibility as principal was to nurture a school climate in which strong, caring, personal relationships could flourish. Because of these assumptions, she engaged in a number of intentional activities meant to promote such an environment. The effect was that teachers enjoyed their work and experienced a strong sense of community (see Figure 12).

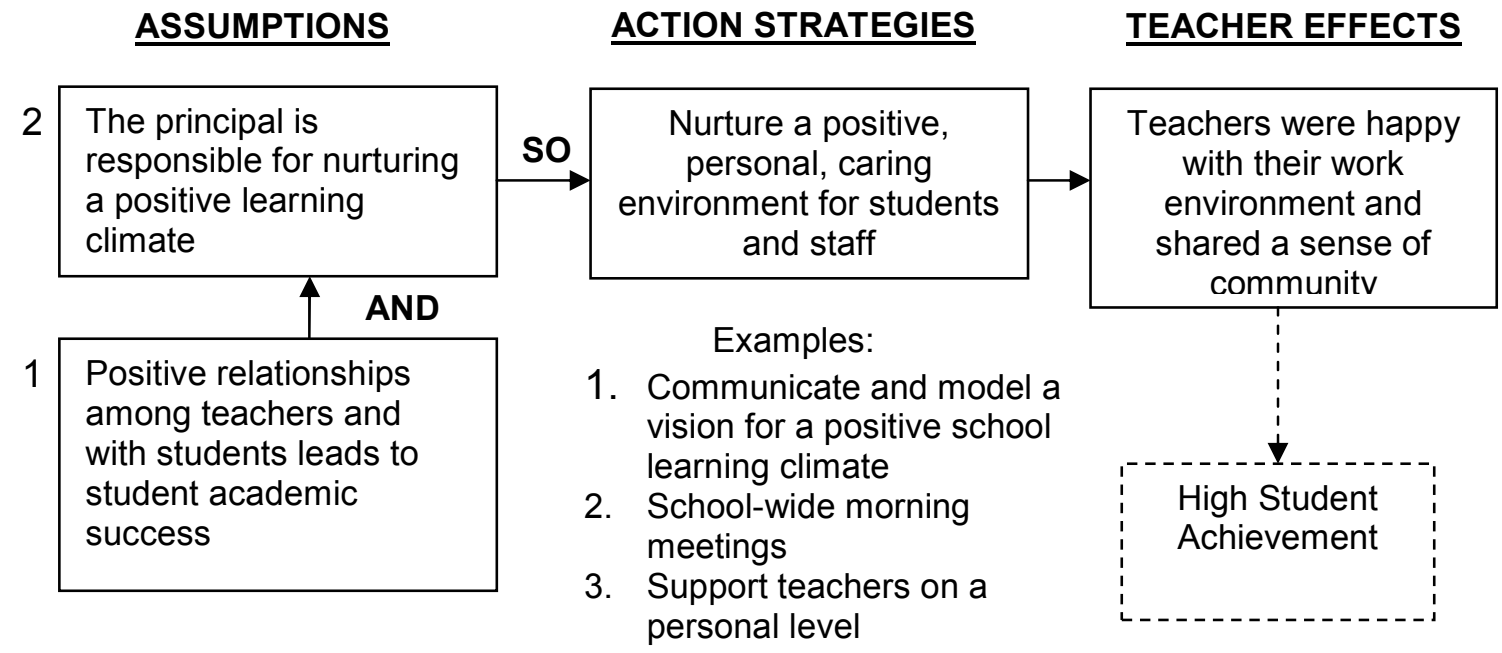

Figure 12. Donna Rippy, Theory of Practice 1, for nurturing a positive learning climate, including assumptions, action strategies and teacher effects. A tacit assumption of all instructional leadership theories of practice is that these action strategies will indirectly result in higher student achievement, though this link is not explored in this study (indicated by dashed lines in the figure).

Assumptions. Ms. Rippy assumed that children learn more and succeed at higher levels when they share a sense of community and when they feel love and affirmation from adults. "I guess to wrap my philosophy up, and this is not the first time I've said it—I say it a lot here—children really don't care how much you know until they know how much you care. That is just it in a nutshell" (DR, 1/22). She understood her primary responsibility as nurturing such an environment at Cherrywood: "If there's one thing I 
do, I help to make a positive learning environment. I've tried to do that with parents, teachers, [and] students. Students are always first and foremost, then the teachers, and then the parents come along. Just [nurturing] that good feeling" (DR, 1/22).

Inspired by the nurturing relationship she had as a teacher with one of her former principals who encouraged her to go into administration and taught her the importance of serving others, Rippy viewed her work as a kind of ministry that focused on the emotional well-being of others and that enriched her own emotional life. Her eyes filled with tears as she described her feelings: "What happens here every day is bigger than me. I'm here for the people I serve. I love them" (DR, 1/22). Mr. Yeager, a physical education teacher Rippy hired as an intern teacher the year Cherrywood opened, agreed that "loving and caring for the people she deals with, whether it's teachers, custodians, [or] cafeteria workers" was Rippy's primary role within the school (SY, 2/1).

Rippy assumed positive relationships with teachers had a ripple effect on the whole school community: "I've always heard that happy teachers make happy kids, and happy kids make happy parents. And for the most part, I think that is reflected here at Cherrywood" (DR, 2/4).

Action Strategies. Because of her assumptions about the importance of establishing strong positive relationships with students and teachers, Ms. Rippy engaged in a number of behaviors meant to foster a warm, loving environment within the school. She described her own relationship with students as an example:

I firmly believe that, and I teach the kids, even when they are in trouble, I tell them the same thing I always told my own son: I may not always like what you do, but I'm always going to love and care about you. I try to get that message across in the way I deal with kids. I basically have an open-door policy. It won't surprise me if while you're here we don't hear some little knocks on the door because they're not accustomed to my door being shut. They come to tell me if 
they think there's somebody doing something they shouldn't be doing, they come in to give cupcakes on their birthdays. We had two dogs in the building on Friday because one little girl was having her birthday and she wanted her two dogs to come. And that's perfectly okay with me. I think it's a very positive [school] environment. (DR, 1/22)

As the first and only principal at Cherrywood Elementary Rippy hired the entire school staff and had the opportunity to shape a school culture in a deeper way than most principals do. Creating a family-like school climate was at the center of her vision for

\section{Cherrywood:}

The goal at that point, and I guess I didn't know any better, was to, number one, have a school where kids wanted to come and enjoy learning. Second, my goal was to have a school where the teachers and staff looked forward to coming and working with kids. Curriculum and all that kind of thing, I knew would happen. Those two things were first and foremost in my mind. (DR, 1/22)

Helen Bowen, a 33-year veteran special education teacher, was one of the teachers Rippy hired to open the school. She related Rippy's efforts to nurture a positive environment to her beliefs about how to best address the challenges of student diversity:

She knew we were serving a very diverse population here. We have it from both ends of the [socio-economic] spectrum, and she knew she wanted to make it work. So from the outset she made it clear that this was going to be a family environment, that she wanted us to be considered a school family. She set the tone for the climate and that was a top priority for her. She wanted all kids to get along, all kids to work together and be together and for us not to know the difference between them [students from different socio-economic backgrounds]. So she stressed that and pretty much made that clear that this was one of her objectives and she made it clear to her staff that was something we would be accomplishing. (HB, 1/25)

"She does want us to be a family," agreed Jenna Wilson, a primary teacher who

taught at Cherrywood for five years. "She wants it to be a family atmosphere and tries to lead us toward that" (JW, 1/30). Mr. Yeager said Rippy used the concept of "family" from her first meetings with the new school staff: "She really stressed family among the kids and the teachers, a very positive school climate. The word 'family' was used a lot. 
And that was extended not only to the kids but among the faculty and staff as well" (SY, 2/1).

Bowen gave other examples of Rippy's efforts to nurture positive relationships with the students, including the school wide meetings held each morning, which Rippy facilitated and used as an opportunity to make announcements, celebrate student successes and build the school's sense of community:

She's very family oriented. She has morning meetings to give pep talks to the kids. I think she acts as a type of cheerleader, role model, to provide a positive environment. She knows the names of almost all the kids save maybe some of the kindergarteners. They all want to go up and giver her hugs. She fosters a nurturing relationship with the students on an individual basis, so they're vested here. They know that people care. (HB, 1/25)

Yeager pointed to the school-wide morning meetings as a forum at which Rippy talked to students about issues of emotional intelligence and social skills that fell outside the state-mandated curriculum. "She's one to constantly be talking to the kids about when to say 'thank you' and 'please' and how to act as a person" (SY, 2/1). Rippy often referred to herself as "Mamma Rippy," and tried to play a mothering role for both students and teachers (SY, 2/1). Her strong personal relationship with students helped build bonds of trust:

She does all those things to show all of them that she cares about them. She may not touch them every single day because there's so many students, but they know who she is and that she cares about them. When she has to deal with things that might be unpleasant, that helps a lot because the kids come in there and they have a respect for her as more than just a principal, but as a mother figure that cares about them and wants the best for them. That's a big part of her leadership with the kids that helps a lot. (SY, 2/1)

During the researcher's observations, Rippy visited each of the school hallways, where children came and went individually and in groups moving from one class or activity to the next. Individual students all stopped to say hello, and several gave her 
hugs. She called each one by name, asked personal questions that revealed she had an intimate knowledge of the students' families and their personal interests, and later shared stories with the researcher about various personal challenges several of the children faced. She introduced one round-faced kindergarten boy and asked him to describe the best thing about Cherrywood Elementary. The boy shrugged and squinted his eyes briefly, as if in thought, then exclaimed, "Ms. Rippy!" She laughed and hugged him and later swore to the researcher that she had no idea what the boy was going to say (Observation, 2/1).

Rippy's nurturing of personal relationships extended to the faculty as well. The school-wide morning meetings played a role in encouraging a sense of community among teachers, according to Mr. Yeager. The meetings "make sure we are seeing each other. I think when you come together every morning, like we did from the beginning, that just lets everybody see who everyone was and just built that family atmosphere a little better" $(\mathrm{SY}, 2 / 1)$.

Rippy's concern for teachers on a human level was evident in her support for teachers' personal problems that affected their work:

I think for the most part, it's as simple as when you call in and you have a child sick. You could be given a hard time about that. For her, she understands that there are situations that are uncontrollable. If you do it the right way and you don't overuse it or abuse it, she is going to help you with that. I can't think of a time when I've called and I've had a hard time about it. It's been, "You take care of your child and we'll see you when you get back." You see that constantly throughout the building with the problems we have, different personal problems. $(\mathrm{SY}, 2 / 1)$

Rippy went out of her way to be supportive of teachers who were in personal crisis: 
I have a teacher right now that hasn't been on her game, but I know also-she didn't come to me, but I finally said, "Is everything okay?" And she said, "Does it show?" And so that opened up a conversation. There were some home problems. I have another teacher who is going through a nasty divorce and she called yesterday and I said, "You tell me what you want to do. Do you want a sub for the whole week?" (DR, 1/22)

Rippy's office reflected her personality and her relationships with students, staff and her own family. During one observation, she was wearing a gray sweater with a small Harley-Davidson emblem on the chest. A mug that read "Harley Mamma" and a picture of Rippy on her motorcycle sat on her desk. The walls were covered with class assignments, artwork, and cards students had given her as gifts. The office was brightly decorated with stuffed animals, figures of bears and other characters, pictures of her family, and country-style crafts. A carved wooden apple on the desk read, "Mamma Rippy” (Observation, 2/1).

She picked up a photograph and showed it to the researcher. "We've had 28 babies [born to school staff] since we opened," she said, pointing to the picture of a large group of teachers and their children.

That's sixteen of them in the photo. We got them all together last year. We had one Friday afternoon. See, I tell them their families come first. I have two more - maybe three more - expecting right now. Maybe one that hasn't been announced. I told them before I retired I wanted them all together. But we managed to get sixteen of them together for this picture. (DR, 2/1)

Effects on Teachers. Ms. Bowen gave Ms. Rippy credit for the school's positive climate and unifying the staff around the desire to make the school a student-friendly environment: "We all made it happen because that's the way she wanted us to go and we were willing to do that and wanted to do that. It was the utmost priority and I think she's been very successful at that" (HB, 1/25). Rippy's encouragement and attention to teachers' personal needs promoted a positive work environment. "I feel really good 
about being here. I think she holds us in high regard. I love working here" (DB, 1/25). Ms. Wilson agreed: "I do feel she's very supportive. She is able to come to bat for you when you're having issues. She will be on your side. ... I think it's very important in that sense to feel that you are appreciated" (JW, 1/30).

Rippy's positive interpersonal relationships with teachers and the nurturing school climate motivated teachers to do their best work, according to Mr. Yeager:

You want to come to work when you feel good. You always have your good days, bad days, you even sometimes go through a funk every once and a while where you may have a month when you feel, "Oh, I just can't do another day." But if you feel like, if there's any place I've got to go to work, this is the one place I want to go, that helps you get up in the morning and get there. You feel more of a dedication too when you work for somebody that you know cares about you more than just ... as an employee. ... I think her caring about everybody makes them want to work harder for her at times, even when it may not be the best day. (SY, 2/1)

Teachers at Cherrywood affirmed the sense of "family" among the staff, in part because Rippy encouraged regular socializing among teachers to nurture a sense of community. "We had a social a couple of weeks ago and any time we have anything like that, everybody from the custodian to the cafeteria people to the bus drivers are invited. We don't make a distinction between, this is a teacher and this is an office aide. Everybody's a part" (DR, 1/22).

During observations, large groups of teachers shared lunch together in the faculty lounge. Laughter and loud conversation filled the hallway outside the room. Rippy teased and joked with cafeteria staff, some of whom she had known personally for many years. In the hallway, a large clutch of teachers gathered to see the newborn baby of another teacher currently on maternity leave. Rippy took her turn holding the infant (Observation, 2/1). Rippy reduced the number of full faculty meetings because teachers 
spent too much time socializing with each other. "I tried everything [to get them focused]," she said, laughing. "I'd give them ten minutes to talk because when you get them all together, they are so glad to see each other from one end of the building to the other, it's just like I can't get control”' (DR, 1/22).

Ms. Bowen linked the positive work culture to student achievement. "I think it's really important. That's one of the reasons for our success in test scores and other areas. You can walk down the hall and everybody's as friendly and open and optimistic with every student and I think it's just made a big difference" (HB, 1/25).

The teachers acknowledged that Rippy's emotional disposition sometimes caused staff members discomfort, but Mr. Yeager admired Rippy's vulnerability and personal authenticity:

Because she does wear her heart on her sleeve, and there are moments when things bother her or she gets excited. She's not afraid to break down and cry in those moments, [and] it bothers some teachers. They want that person who is not going to do that... Funny thing is, when teachers are having problems or emotions in their lives, they want her to be accepting of that. . She is human.... I think over the years she's realized that people get uncomfortable with that emotional thing, so you can tell she's tried to not show it as much. But I don't think it's a bad thing. For me, it's who she is and you either accept her or not, but at least she's being who she is. (SY, 2/1)

In summary, Donna Rippy's first theory of practice was based on the assumption that positive relationships with and among teachers and students was the key to increasing student achievement. A number of action strategies emerged from this assumption, including communicating a vision that school should function like a "family," using school-wide morning meetings to celebrate student success and emphasize issues related to emotional intelligence, and being personally supportive and concerned for teachers. Teachers at Cherrywood confirmed the importance of this theory 
of practice to Rippy's leadership, and valued the sense of community and positive learning climate the principal had nurtured at the school. Teachers credited this learning climate for the school's high level of achievement.

\section{Theory of Practice 2: Inviting Teacher Input}

Ms. Rippy spent her entire career at the high school level prior to becoming principal at Cherrywood. As a result, she assumed she needed to rely heavily on the input of teachers and her leadership team, and actively tried to engage others in the decision-making process. This led the teachers to collaborate with the leadership team in various ways. Data suggested this was a relatively new theory of practice for Rippy, who relied on other assumptions and action strategies earlier in her career as principal (see

Figure 13).

\section{ASSUMPTIONS}

2

2 Teachers can offer valuable input to decision making

\section{AND}

1 The principal does not have all the answers for increasing student achievement
ACTION STRATEGIES

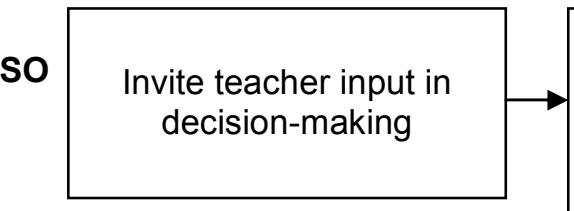

\section{Examples:}

1. Rely on input of teachers and administrative team

2. Delegate leadership responsibilities to others
TEACHER EFFECTS

Teachers collaborate with the leadership team to make decisions and feel empowered

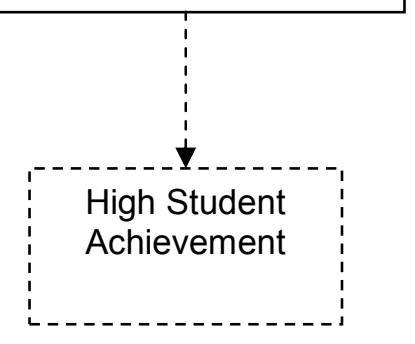

Figure 13. Donna Rippy, Theory of Practice 2, for inviting teacher input, including assumptions, action strategies and teacher effects. A tacit assumption of all instructional leadership theories of practice is that these action strategies will indirectly result in higher student achievement, though this link is not explored in this study (indicated by dashed lines in the figure). 
Assumptions. Rippy acknowledged she was not an expert on elementary

education, curriculum or instructional strategies but could rely on the wisdom and experience of teachers. "I look at myself as the leader of the building, but I don't look at myself as being all-knowing" (DR, 1/22). She described herself as a "facilitator" from her first year as principal at Cherrywood, Mr. Yeager reported. "She always says, 'You're the experts. I'm here to help you and get for you what it is you need to make your job easier or help you do your job better"” (SY, 2/1).

Rippy invited teacher input because she believed teachers who were a part of decision-making would experience higher levels of job satisfaction and motivation, and because she relied on their expertise and experience as classroom teachers to inform her own leadership decisions:

If you don't feel valued then you have a lot of second thoughts as to why I'm doing what I'm doing. I believe that people have more buy-in if they're allowed to have input. I believe when people see that you value what they think or how they feel and you take those things into consideration, it's a leg of that positive climate. A lot of administrators lead by simply being a boss. That's not my nature. I invite that teacher input because I do look at them as being the professionals. They're the ones that deal with it day in and day out. A lot of my job is looking at the big picture and making sure those needs are met. They're there and they're doing it. They have a lot of things to teach me. (DR, 3/12)

Action Strategies. Because Ms. Rippy viewed herself as a facilitator and not an expert, especially in matters related to elementary education, she engaged in a number of behaviors meant to encourage teacher input and participation in decision making. Depending on others also gave her the freedom to focus more on her main priorityschool climate:

I'll tell you what I tell people: I'm circled by a lot of wonderful, knowledgeable people here. I have a curriculum coordinator who is fantastic. I have a guidance counselor who has a calling. I laugh sometimes and tell people, "They do all the work and I just love the kids." (DR, 1/22) 
Rippy engaged the faculty in collaborating with one another and supporting her with ideas and feedback for decision making, such as the hiring of new teachers (DR, 1/22; JW, 1/30). For difficult situations, she relied heavily on her leadership team, composed of the school's curriculum coordinator, guidance counselor, and assistant principal: "If I am reluctant about making a decision, I'll get my team up here ... and I'll say, 'What do you all think about this?' because they've been in the classroom more recently than I've been in the classroom. So we'll bat things around like that" (DR, 1/22). She described a reciprocal trust and respect she shared with the teachers and gave examples of how she invited them to advise her and participate in decision-making:

I think the teachers want us to be the best and they know I want our children to be successful. I think they trust me to make decisions, but I also ask them to be a part. I never have an interview [with a prospective teaching candidate] that I don't use a team. I had a team back here earlier today because we've just hired a new family resource coordinator, so I said, "Come in and help me get the resource center set up." The custodian was here. How can we do this? So it's a team approach and I do ask for their input. I've continued to learn and I continue to learn from these people. (DR, 1/22)

Ms. Bowen confirmed that Rippy depended on the experience of teachers to help

her with elementary-level problems:

She relies heavily on those that have been in elementary all their careers, like our curriculum consultant, like our guidance counselor, like some of the team leaders. I think she relies on them to fill in some of the voids that she doesn't have as a result of her high school experience. I don't think she would come in and tell a kindergarten teacher how to do something unless-I think she would seek out advice from the other people who knew.... So she relies on others to help her through that. Now, when it comes down to it, she's willing to make the decision, but she just doesn't come in and make decisions without consulting people who have had the elementary experience, even today. Because let's face it, she's been a principal all these years in an elementary school, but she hasn't been a teacher in an elementary school. She still hasn't taught reading to first graders or math to sixth graders. (HB, 1/25) 
"She is able to delegate out different things she feels would be better handled by someone else," reported Ms. Wilson. "She doesn't try to do it all on her own" (JW, 1/30). Grade-level teacher teams at Cherrywood did not have formal team leaders, and Rippy called on various members of the team to advise her or take responsibility for various projects depending on their personal interests and areas of professional knowledge:

I am on the math learning team, and involved in the Math Alliance [an on-going professional development program sponsored by the local educational cooperative], so a lot of times if it's something that has to do with math, she might come to me. She went to Ms. Grover recently because she was on the original Thoughtful Classroom team and we had a team meeting on that topic recently, so she was the one who led that up. It's good because everybody feels like they are a little more a part. (JW, 1/30)

Rippy facilitated a meeting in her office illustrating her solicitation of teacher input for decision-making. She met with the lead preschool teacher and the district's preschool consultant to discuss which kindergarten classrooms special needs preschoolers should attend the following school year. The trio discussed each child individually. Rippy asked multiple questions about the children's needs, the attitudes and involvement of their parents, and the nature of their conditions. Together, the group made recommendations for which kindergarten teachers would be most adept at meeting the needs of specific children. At the end of the meeting, Rippy asked about whether the preschool teacher and consultant were interested in establishing an all-day preschool program in coming years (the school offered two sessions of half-day preschool). The preschool teacher noted the school could apply for a federal grant to fund the program. Ms. Rippy expressed her support of the idea if the preschool staff was interested. "If that's what you want to do, we'll do it" (Observation, 2/1). 
Effects on Teachers. Teachers at Cherrywood reported a sense of empowerment and motivation because Ms. Rippy invited their input and participation in decision making. Mr. Young connected this effect to the overall sense of community the teachers shared: "Teachers are motivated by the feeling of being a part of what takes place in our school" (SY, e-mail correspondence, 2/26).

In sum, Rippy's second theory of practice emerged from her assumption that to be an effective instructional leader, she needed to rely on the wisdom and experience of classroom teachers, especially since she had no prior experience in elementary education. Teachers who were invited to participate in decision making would have higher levels of empowerment and job satisfaction. As a result, Rippy described herself as a "facilitator," encouraged teachers to collaborate, and solicited teacher feedback. Teachers confirmed a sense of empowerment and motivation because of Rippy's theory of practice.

\section{Theory of Practice 3: Giving Teachers Autonomy}

Ms. Rippy's practice of giving teachers autonomy was closely linked to her theory of practice of inviting teacher input. Rippy assumed that if she hired quality teachers, she could trust them to do their jobs with only a modicum of directives. Teachers responded to this autonomy by expressing a sense of appreciation and freedom (See Figure 14).

Assumptions. Starting with the same assumption as Theory of Practice 2, that as principal she held no more intrinsic knowledge about teaching than any one else, Ms. Rippy trusted teachers to experiment, innovate and carry out their jobs largely as they pleased. She linked this to an assumption that a bond of mutual trust and professional respect was critical for a successful school environment: 


\section{ASSUMPTIONS}

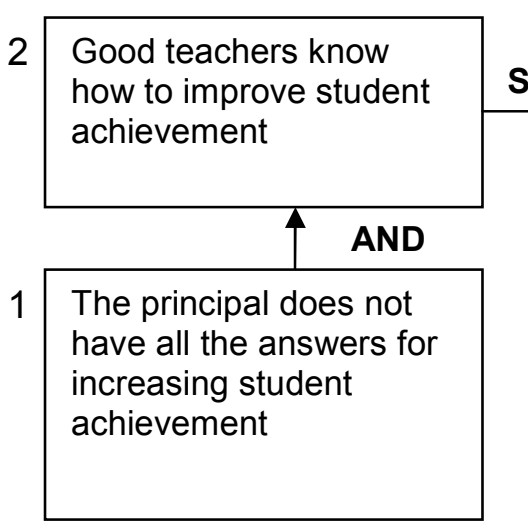

ACTION STRATEGIES

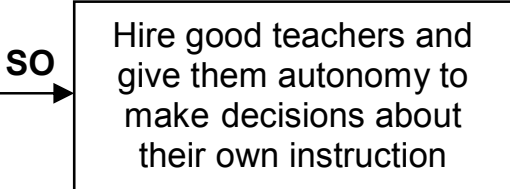

\section{TEACHER EFFECTS}

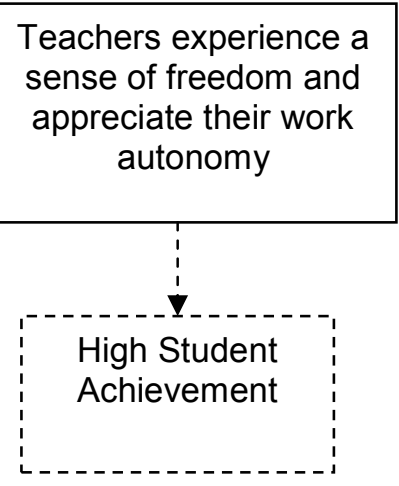

Figure 14. Donna Rippy, Theory of Practice 3, for giving teachers autonomy, including assumptions, action strategies and teacher effects. A tacit assumption of all instructional leadership theories of practice is that these action strategies will indirectly result in higher student achievement, though this link is not explored in this study (indicated by dashed lines in the figure).

I want teachers to feel like they can trust me and I want to feel like I can trust them. .. . They have to know that you believe in them, that you value their input, and you have to give them that leeway to try new things and venture outside that box and not fault them for it if some part of it fails. (DR, 3/12)

She believed that without a sense of autonomy, teachers would view their work more as a "job" and less as a vocation. "I think it would be the same song and dance, day in and day out, year in and year out, with no real capacity to grow or 'want to' to grow and be better" (DR, 3/12).

Action Strategies. Ms. Bowen was appreciative of the autonomy Rippy allowed her staff:

I think she's good at picking good people when new staff are coming on board and then giving them the freedom to do what they do best. I don't think she's a micromanager in many respects. She lets the guidance counselor do what the guidance counselor does. She lets the special ed department do what we know best, realizing that special ed was not an area she knew a lot about as a business teacher or assistant principal. ... She does realize she's got some good people. Let's use their knowledge and judgment and go from there. (HB, 1/25) 
Mr. Yeager described Rippy as a facilitator rather than a micromanager. While her approach was not completely hands-off, successful teachers knew she was going to encourage their autonomy:

So for her, if you're doing your job, she's not going to micro-manage. She's not going to be in the classroom every second of the day checking. If you need help, she's going to be there to help you and if there's times you might not be doing the things you need to do, she's going to let you know. But she just doesn't micromanage. To me, that's a big thing, her being that facilitator that I know I can go to, but at the same time be the teacher I want to be and do my job the way I want to do it. (SY, 2/1)

Effects on Teachers. Faculty expressed appreciation for Ms. Rippy's trust in their work. "I can go to her, but at the same time I can be the teacher I want to be and do my job the way I want to do it," Mr. Yeager said (SY, 2/1). Ms. Bowen described the effect as a sense of freedom.

I think she gives them [teachers] the freedom [to do their jobs as they see fit]. They don't get the feeling, unless you are having some struggles, that she's staring at your back or looking over your shoulder. I think she gives them the freedom to do what they know best. And we have some excellent experienced teachers that help with others on their teams. I basically think that's it. She just does stay out of the way unless she needs to intervene. ... Donna lets us do what we do best. (HB, 1/25)

Theory of Practice 3 emerged from Rippy's assumption that she was not an expert and therefore had to give teachers wide latitude in determining how to do their work for the school to be successful. Her action strategy for this theory of practice was to encourage teachers to experiment and be innovative and understand themselves to be professionals. Teachers expressed appreciation for the sense of freedom that Rippy's theory of practice evoked in them. 
Theory of Practice 4: Encouraging Teacher Collaboration

Ms. Rippy believed teacher collaboration leads to school effectiveness, so she strongly emphasized the importance of teachers working together to plan, learn and reflect on student outcomes. Teachers responded to this by actively engaging in teamwork and collaboration (see Figure 15).

ASSUMPTIONS

$3 \begin{aligned} & \text { Teachers will not } \\ & \text { engage in collaboration } \\ & \text { with orchestration on the } \\ & \text { part of the principal }\end{aligned}$

AND

2 Educators must engage in ongoing professional learning; this is often best achieved by learning collaboratively

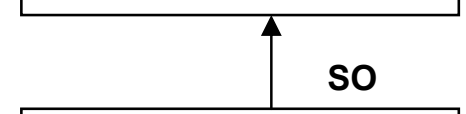

1 The challenges of improving student achievement are too complex for any single educator to have a definitive plan for student success.

\section{ACTION STRATEGIES}

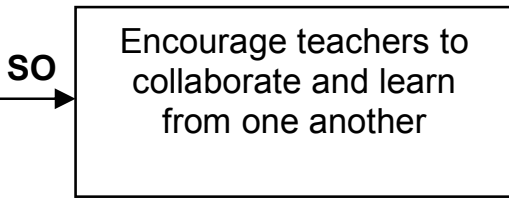

1. Place teachers in multigrade level teams for professional development and sharing 2. Host semi-yearly meetings with grade level teams to share student information with next grade level

3. Encourage teacher collaboration on innovative instructional approaches

\section{TEACHER EFFECTS}

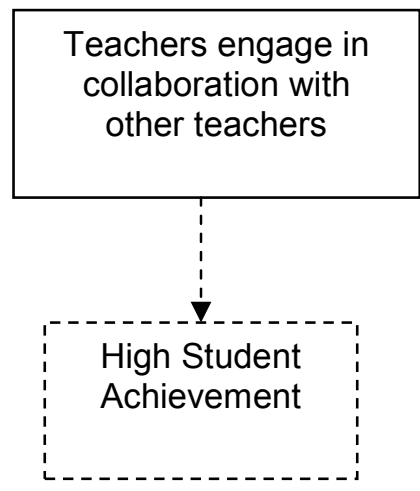

Figure 15. Donna Rippy, Theory of Practice 4, for encouraging teacher collaboration, including assumptions, action strategies and teacher effects. A tacit assumption of all instructional leadership theories of practice is that these action strategies will indirectly result in higher student achievement, though this link is not explored in this study (indicated by dashed lines in the figure).

\section{Assumptions. Ms. Rippy believed the challenges of improving student}

achievement were too complex for a single educator to have a definitive plan for student success, so educators must engage in ongoing professional learning. This was often 
best achieved by teachers learning collaboratively and she saw her role as facilitating and encouraging such shared learning:

Just as we all have different learning styles, we all have different teaching styles. What might work for one teacher would not work for another if they're just tuned into that. I think we all learn from one another.... I've observed a lot of schools where the majority of teachers are islands unto themselves. I think some teachers prefer it that way, but if you build that culture and climate that we're all in this together and I may not have all the answers but somebody down the hall might, or I may have worked with this type of child before and you may never have had that experience, then you share and learn. We all have different life experiences, we all have different teaching experiences, we do have to look at it that we're all in this together. (DR, 3/12)

Action Strategies. Ms. Rippy promoted teacher collaboration by intentionally arranging teachers in grade-level and cross-grade-level teams that met regularly to discuss student needs, analyze student achievement and learn new instructional strategies. According to Mr. Yeager, she emphasized the benefits of learning from one another. "She uses the words 'team player' a lot and that's kind of inspired [us]. It's a motivation thing. It clicks in your mind that everybody has to work together" (SY, 2/1).

Teacher collaboration is a normal expectation of the school's culture, according to Ms. Bowen. "She has really pushed for the collaboration among teachers. We're going to have team meetings. ... We're going to get together and share ideas. It's the best school I've been in, in terms of special education staff working together" (HB, 1/25). Seven learning clubs met twice monthly as a part of the Thoughtful Classroom initiative and "a lot of conversation takes place there" (DR, 1/22). Rippy, the curriculum coordinator, and the assistant principal rotated among the learning clubs during meetings to monitor, observe and participate in the dialogue. Yeager described how Rippy's involvement with the learning clubs encouraged teacher participation: 
She very much promotes that and with all the different meetings that I've been at, she's always here and there and moving around [among the teams]. She comes in and sits with groups for a certain amount of time and listens to the things that are going on. You're actually seeing her face and she's not just sitting in her office while everybody else is meeting. (SY, 2/1)

The learning clubs were vertically aligned, with teachers representing a variety of grade levels. Rippy saw this as an opportunity to encourage deeper teacher dialogue about student work and instructional strategies:

When we're vertical, the teachers actually bring student work [to review with their team members]. To see a sixth grade teacher look at spelling on the first grade level, it's amazing. To see how they adapt those teaching strategies, that's the most powerful thing we've done is to have those learning clubs where that sharing takes place from peer to peer. (DR, 1/22)

Rippy invited the teachers from each grade level to meet with her twice yearly

and discuss student needs and the transition of students from one grade level to the next:

I'll bring in the sixth grade teachers and say, "Tell me what I need to tell the fifth grade teachers" [about your expectations for their students]. And then I'll bring the fifth grade teachers in and say, "Tell me what I need to tell the fourth grade teachers." ... That's pretty powerful too. Just the atmosphere of knowing. The team I had in here a few days ago, they said, "We're a well-balanced team because of my downfall is her strength." So they recognize that in their teams. (DR, 1/22)

Teachers at Cherrywood initiated the practice of looping, in which teachers taught the same students two years in a row. One team of teachers taught first and second grade, and another taught third and fourth, allowing them to know the students better and meet their needs more effectively. "It's very important that we're able to communicate well from our team to the next team these students go to," Ms. Wilson explained. "Because we have them for two years and the next team has them for two years, so I do feel like there is good communication, especially from our teams" (JW, 1/30). 
Rippy described another team of teachers who initiated the idea of administering formative assessments every two weeks, and then continuously regrouping students based on their progress. Rippy expressed skepticism about the logistics of regrouping students so often, but encouraged the teachers to try the practice anyway: "It worked and they continued to do that. I want them to look at different ways we can serve our kids. If they can make it work and they can do the work to make it work, I'll listen and support them" $(\mathrm{DR}, 1 / 22)$.

Mr. Yeager noted that Rippy emphasized the importance of teams the summer before the school opened, and asked the faculty to participate in a ropes course and other team-building activities. "I had been in the military for a few years, so I knew right off the bat that those are the kinds of things you have to do to build that camaraderie and get people to trust each other" (SY, 2/1). Yeager considered Rippy's collaboration with her leadership team a model of her expectations for teacher teamwork:

I see her and Darla [the curriculum coordinator] working together all the time on different things. ... What I see from her, she's not one that would sit there and say, "I know it all and I'm not going to listen, I'm going to do my own thing." With Sandra [the former ECC] and now with Darla, they come to her with ideas, whether it's open response questions or using a rewards system - when it comes to curriculum, she's very open to listening to them and trying new ideas, doing new things. (SY, 2/1)

Effects on Teachers. Ms. Rippy and teacher participants gave abundant examples of teachers engaging in teamwork and collaboration. Three teachers recently approached Rippy and asked to write the math curriculum for the second grade. Mathematics was one of the subjects Rippy had identified as an academic growth area for the school:

I said, "Go for it." So then I gave them two days to work on it. They worked on it in the building and then they worked on it at somebody's house but they brought back the "I can" statements [converting state standards into student- 
friendly statements that describe the specific skills or knowledge the students should acquire]. Oh, man, was it good. (DR, 1/22)

"I love the fact that teachers here are hard working and that they're willing to collaborate with me as a special educator," Ms. Bowen reported.

That's really important. I've walked into schools where it was already set up, the climate between special education and regular education was an adversarial one. . . I never felt comfortable. Here, none of that holds true. It's a great place to be. . .. Obviously, our test results show it, but it's more than test results. It's walking down the hall and realizing how everybody gets along. (HB, 1/25)

Mr. Yeager especially valued team meetings because of "the idea sharing." $\mathrm{He}$ learned from the experiences of other teachers and was able to adapt more instructional strategies to his PE classroom environment:

There's constant collaboration that trickles down through all that. When I come in, coming from a completely different classroom atmosphere and environment .. . there are things [instructional strategies] what would be very difficult for me to do in a 45-minute period, but it allows me to see the idea and see what's going on and know what kids are doing in the classroom. It allows me to take some of those ideas and use them and change them up if I need to. But also just by collaborating with those teachers, I think it goes back to the whole family atmosphere and creating a positive learning environment. (SY, 2/1)

Rippy's fourth theory of practice emerged from her assumption that the challenges of improving student achievement were too complex for any single educator to overcome alone. She assumed teachers learned best when they learned from one another, and understood her role as principal to involve facilitating that collaboration. She used action strategies such as placing teachers in multi-grade level teams for professional development and sharing, and encouraging teachers to work together in learning and exploring new instructional strategies. The effect on teachers was that faculty at Cherrywood embraced collaboration and frequently partnered with one another. 


\section{Theory of Practice 5: Maintaining a Focus}

A strong feature in teacher and principal interviews was Ms. Rippy's conviction that all children can learn and achieve academically, regardless of their language, socioeconomic background or other barriers. She assumed that teachers have a great responsibility for bringing out this potential in children, especially for preparing them for the learning challenges they would face as middle and high school students. This assumption translated into an emphasis on utilizing a common curriculum and providing students learning tasks aligned to their unique learning styles and cognitive needs. Rippy believed it was her responsibility as principal to help teachers maintain their focus on teaching the established curriculum and simultaneously using student-centered instructional strategies. She engaged in specific behaviors designed to assist teachers in maintaining this focus, and teachers reported a strong sense of responsibility for student outcomes as a result (see Figure 16).

Assumptions. Ms. Rippy said her experiences at Cherrywood taught her that poverty and language were not barriers to student achievement. Teachers quickly identified this assumption as one of Rippy's core beliefs about learning, and described how it shaped much of her leadership behavior including both academic concerns and student discipline.

"We have $36 \%$ of our students on free and reduced lunch, but the beauty is when you walk around the school you can can't tell the difference [between them and students from more affluent homes]," Rippy reported (DR, 1/22).

Ms. Bowen, confirmed that Rippy believed in the possibility of every child's success and the obligation educators face as a result: 


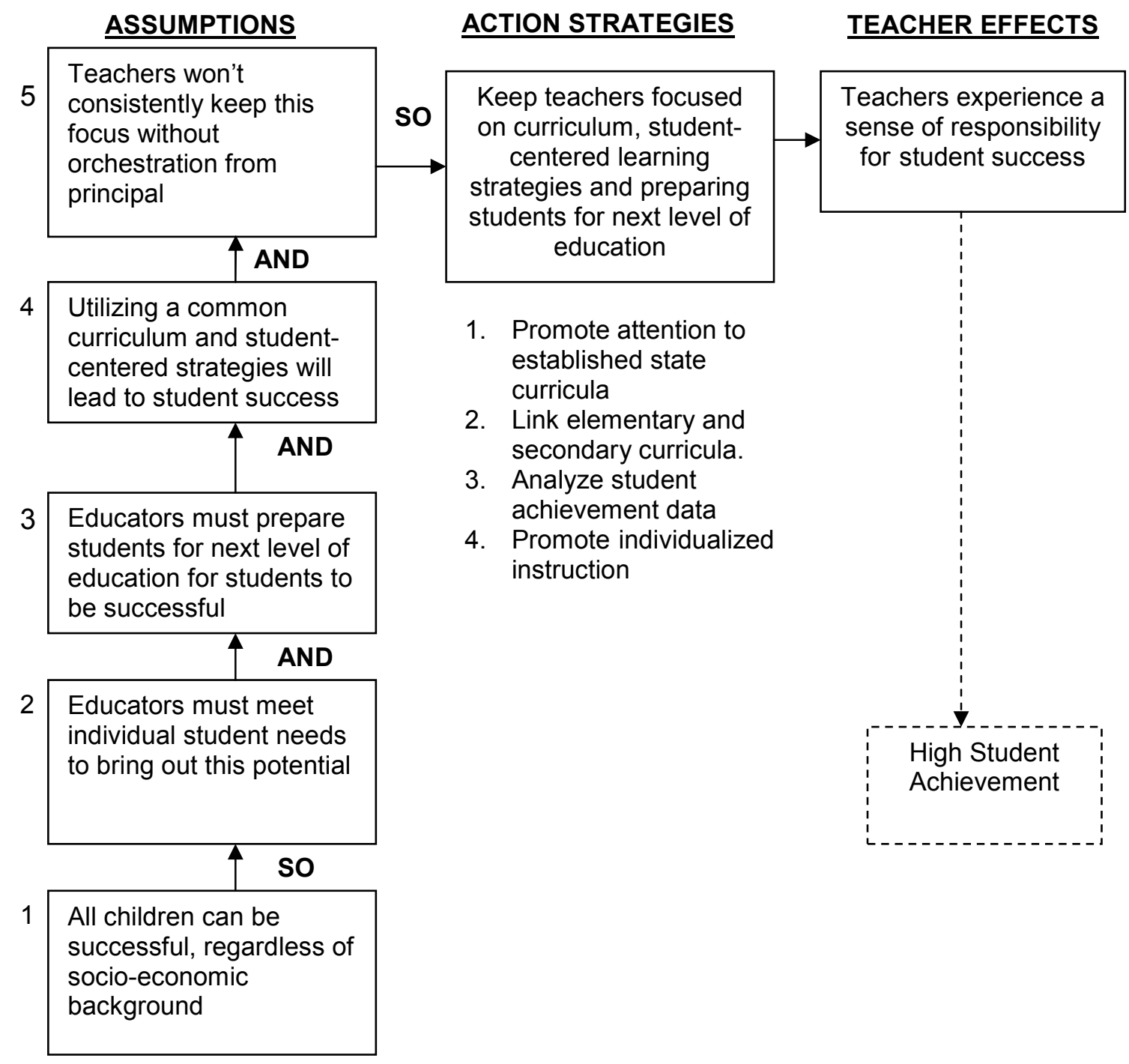

Figure 16. Donna Rippy, Theory of Practice 5, for maintaining a school-wide focus on curriculum and instructional improvement, including assumptions, action strategies and teacher effects. A tacit assumption of all instructional leadership theories of practice is that these action strategies will indirectly result in higher student achievement, though this link is not explored in this study (indicated by dashed lines in the figure).

One of her core beliefs is that every child can and will learn in her school. Every child has potential and it's our job to tap that potential. And it will be tapped.

She believes in the innate ability of each child to make progress and to learn. She believes teaching is an awesome responsibility [because of that]. (HB, 1/25)

Mr. Yeager pointed out this assumption's instructional implications: 
She definitely believes that every child can learn. She understands the backgrounds of the kids and that every kid can learn and at the same time, there's going to be a lot of differences in the way they learn and the way we have to approach them instructionally. (SY, 2/1).

Ms. Wilson said Rippy's emphasis on each individual student's potential was exemplified in her belief that students should not get equal treatment, either academically or behaviorally, because each student has unique needs. "One things she often says is that, 'Fair is not equal.' I know she thinks everyone can learn and so each student has to be approached differently" (JW, 1/30).

Action Strategies. Ms. Rippy believed she was responsible for maintaining a strong school-wide focus on student achievement, including making sure teachers taught the state-mandated core content and differentiated instruction for individual student needs. Drawing on her high school experience, Rippy also took a broad view of the elementary school's mission, focusing on preparing students not just for elementary achievement tests, but also for their secondary education.

The traditional culture of teacher autonomy made unifying the staff around a common curriculum challenging, but Rippy believed her role involved helping teachers understand their work in new ways:

I came from a time when so many times you'd have a veteran teacher down in the primary area, and we'd have a hard time getting her away from a month [of teaching] on dinosaurs. I think just shifting of paradigms is hard for teachers because that's their four walls, their door to shut, and I'm going to teach this just because I like to teach it. I think [of my role as] being a guiding force and really getting those teachers to look at what national standards are and what we need to be teaching these children so they will be successful in middle and high school. You know, high school always blames middle, and middle school always blames elementary [for students being unprepared]. I don't want us to be a school they are going to blame. I want them coming back to us from the middle school saying, "Cherrywood kids are always prepared." (DR, 1/22) 
Her emphasis on meeting the needs of individual children also shaped Rippy's understanding of her role in guaranteeing that teachers differentiate their teaching: "You have to always constantly change things to reach the children you currently have. You have to differentiate instruction. No longer can we teach to the masses" (DR, 1/22). She believed that elementary teachers were more adept at differentiation than her former high school colleagues were, but there was still an opportunity for elementary teachers to do better in this regard:

It's always bothered me, where do we kill the love of learning? I said that from early on, I have to figure this out. Where do we kill that? And I see some of that in this building going from so much hands-on learning [in the primary grades] to "sage on the stage" down around fifth and sixth grade. Some of those things have changed, but that's what we have to continue to push. (DR, 1/22)

In line with her efforts to prepare students for secondary school, Rippy sought to broaden the school's curricular focus to include standards aligned with college readiness exams such as the PLAN and ACT tests, which were included as a component of Kentucky high schools' accountability index. She also contemplated including more components of the state's Program of Studies (broad curriculum concepts not included in the standardized core content) in the school's instructional program:

Curriculum is ever changing. We're at a crossroads right now. We're trying to decide if we're going with the new ACT push. We're trying to decide if we're going to continue to let the Core Content be our driving force, or are we going to let the Program of Studies be our guide. So it's ever changing, with a lot of legislation coming down, especially with the ACT, and that falls into preparing students for the next level. We're taking initiative in our county - and we always have - but countywide our sixth grade teachers are going to work more closely with the middle school teachers to make sure our students are coming to them as prepared as they can be for their ability level. (DR, 2/1)

Rippy used test data analysis and informal conversation to maintain this schoolwide focus on improving student achievement through mindfulness to the state- 
established core content and diversified instructional strategies. Ms. Bowen confirmed

that Rippy's conviction that all students can and will make academic progress:

She takes a look at test results, like the GRADE, the GMADE. She talks to Title I and gets data to point out how many kids in our school are on grade level. She's in contact with the teachers just on a conversational level. "How's this one doing? How's that one doing?" Kind of following through to make sure the data supports what she believes is going on and if not, then she goes to individual teachers and says, "Okay, we've got to fix this. We have to work on it." (HB, $1 / 25)$

Rippy also emphasized teacher responsibility for student outcomes, according to

Ms. Wilson:

Just with the testing and the scores and how we approach our learning and enforcing with us as teachers that it's our responsibility to makes sure we're addressing all the different avenues as far as making sure we know who the [special] needs [students] are and that we're using different instructional strategies. (JW, 1/30)

According to Mr. Yeager, Rippy placed a strong focus on individualizing instruction: "She has a strong understanding that the students are little human beings and they're not just robots that we're going to do the same thing [instructionally] all the time with them" (SY, 2/1).

Consistent with her other assumptions and action strategies, Rippy also utilized teacher teamwork as a means of promoting higher-quality instruction. A team of math teachers from Cherrywood participated in an on-going professional development program called the Math Alliance, sponsored by the local educational cooperative, with Rippy's encouragement and support. "I give them release time to work on things they need to do. I'll get them a sub and I give them that release time and they've brought a lot of things back to our faculty and staff as far as teaching strategies" (DR, 1/22). 
Despite her efforts to promote curriculum and instruction, Rippy readily admitted that she depended primarily on her curriculum coordinator to assist with those elements of leadership. Her preference was to focus on the school's climate and her relationship with staff and students:

I've had to grow too in order to gain the respect of teachers as far as being an instructional leader. And I don't think I'm there yet. I don't know that I'll ever be there because of my make up. I had much rather focus on the kids and creating an environment than necessarily be deeply engrained in the curriculum.... So whether it's by choice or by nature, I don't know, but I'd just rather concentrate on the relationships because I'm a people person and I love to be with the kids and the teachers. (DR, 2/1)

Effects on Teachers. Teachers at Cherrywood responded to Ms. Rippy's

leadership with a sense of responsibility for student success and a willingness to work

hard. Ms. Bowen credited Rippy's expectations and empowerment of teachers:

The teachers realize there is accountability. ... From a teacher's standpoint, you know the expectations are there, so we are to perform. But the good teachers she has would do a good job whether she was paying attention or not. Our teachers here are that good. We have a great staff and she would be the first to tell you that. It works from the top down... The effect on the teacher is they realize they have her support and they are going to go with it. They work hard. They want to get it done. They know they have to get it done. (HB, 1/25)

Her effort to maintain a school-wide focus on curriculum and instruction "gives Ms. Rippy credibility as an instructional leader," according to Mr. Young. "Teachers' behaviors follow her lead in this area" (SY, 2/26).

Theory of Practice 5 emerged from Rippy's assumption that all children can be successful academically, regardless of their socio-economic background, and her conviction that teachers had to maintain a constant focus on a common curriculum and high-quality, student-centered instruction, to bring about high student achievement. Her action strategies included promoting the use of the state established curriculum, 
intentionally linking the elementary and secondary education curricula, and promoting individualized instruction. As a result, teachers perceived that they were responsible for student outcomes.

\section{Theory of Practice 6: Providing Feedback}

Ms. Rippy believed teachers needed feedback to enhance and refine their instructional practice and to meet their responsibilities in helping all students be successful. Providing feedback was one of her self-perceived roles as principal. Because she believed positive relationships were critical to school success, Rippy preferred non-threatening forms of feedback like engaging teachers in conversation, modeling and making suggestions. These behaviors seemed to correspond with teacher responsibility toward student success. However, in cases when these non-threatening forms of encouragement were not effective, Rippy used more corrective and coercive strategies, such as verbally correcting teachers, placing teachers on corrective action plans, and in one case, non-renewing an untenured teacher (see Figure 17).

Assumptions. A key assumption underlying this theory of practice, and linked to

other theories of practice Ms. Rippy used, was her concern about the dangers of utilizing her positional power too forcefully. Rippy worried that a principal who was too directive could damage her relationship and reputation with others. "I think power can be a deadly thing in a position such as this. I've seen some principals whose reputations were destroyed by being all-knowing, 'This is who I am, you are going to listen to me' kind of thing. Not necessarily destroyed, but they did not have the integrity and reputation [that I desire for myself]" (DR, 2/4). Consequently, Rippy focused on feedback strategies that were more aligned to her practices of nurturing positive interpersonal relationships, 


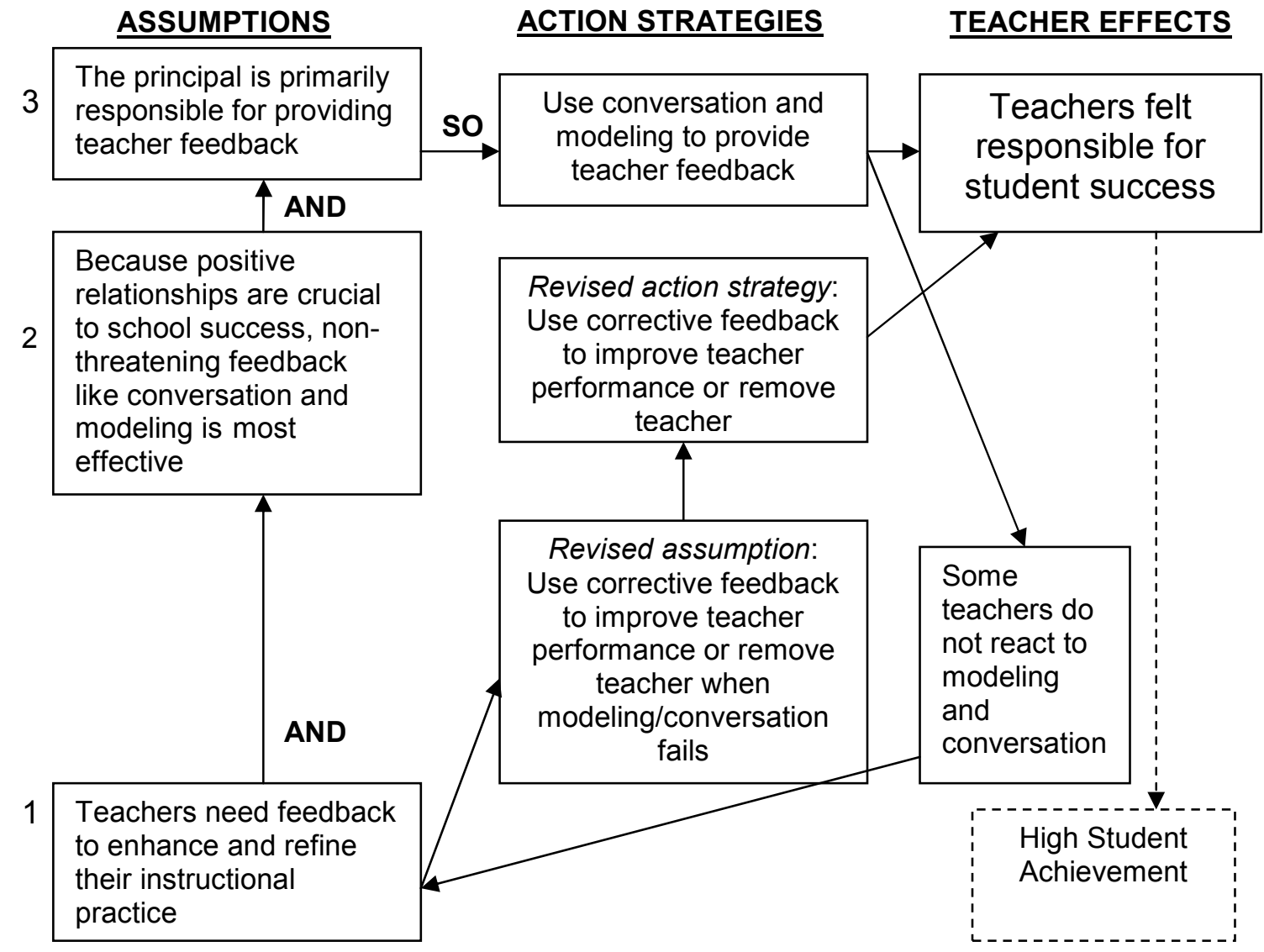

Figure 17. Donna Rippy, Theory of Practice 6, for providing feedback, including assumptions, action strategies and teacher effects. A tacit assumption of all instructional leadership theories of practice is that these action strategies will indirectly result in higher student achievement, though this link is not explored in this study (indicated by dashed lines in the figure).

including using modeling and encouragement to promote teaching excellence. When teachers did not respond to these non-threatening approaches, Rippy used more corrective forms of feedback, even when it was uncomfortable for her. In these cases, her responsibility to students trumped her normal approach to dealing with teachers.

Action Strategies. Ms. Rippy provided feedback to both teachers and parents in the form of suggestions, usually in collaboration with her leadership team or with the teachers themselves (reflected in her use of the word "we" in the following statement): 
A lot of times when we have parent meetings or teachers sit down in here [to discuss instructional problems], we offer suggestions. We offer things for them to try, mainly through conversation and modeling. A lot of times in parent meetings, we will talk about, "Well, I can do this accommodation. I can give them 10 spelling words instead of 20 . I can put them on the computer using this program." (DR, 1/22)

According to Ms. Bowen, Rippy’s non-threatening approach to providing feedback, including her collaboration with the curriculum coordinator, increased teacher self confidence:

She comes in and observes. She makes informal walkthroughs. She relies on the curriculum coordinator to be paying attention to those kinds of things and be reporting any concerns. She uses her eyes and ears and pays attention. She listens. She trusts her teachers, though. It's not a checking up on you process every week. She trusts that you are going to do your job .... which is a safe environment for a teacher who is competent. (HB, 1/25)

Rippy also utilized teacher team structures to ensure that teachers gave each other feedback on their work. Cherrywood teachers participated in the Thoughtful Classroom initiative, a district-wide program in which teachers met regularly in small groups called 'learning clubs' to learn about, experiment with, and share the results of research-based instructional strategies. According to Ms. Wilson, Rippy promoted the learning clubs as a means of keeping teachers focused on improving their teaching. "There is not as much specific individuality [in Ms. Rippy's feedback], how are you doing as an individual, but probably more as a team. You know, her message is this is what your learning club is meeting for today, this is what we're supposed to be incorporating in our lessons" (JW, $1 / 30)$.

"They learn from one another" in these team settings, Rippy explained. "We have team meetings quite often during their planning periods where we say, 'What can we help 
you with? What do you need that you might not have in your classroom? What can I get for you?"' (DR, 1/22).

Despite her emphasis on maintaining positive relationships and her reluctance to hurt teachers' feelings, Rippy used corrective feedback when a teacher's behavior threatened to undermine student learning or the collaborative work environment among staff. She described her decision to non-renew an untenured teacher the previous year.

I let a teacher go last year after four years before she got tenure because I'd given her two years and she still was not performing. That was hard. But I had to think about my students. My babies aren't going to go through that again. Planning [was her problem]. It was all fly by the seat of her pants. And even the second day I was going back to evaluate her, she came in that morning and said, "Can we put it off until this afternoon?" I thought, "What is going on?" Come to find out, she went to my ECC [elementary curriculum coordinator] and said, "She's coming this afternoon. You've got to help me pull together a lesson." And I knew that was happening. I gave her every opportunity to change it." (DR, 1/22)

Rippy described another teacher she was currently working with, a first-year

intern who was not functioning well with the other members of her team:

She thinks she knows it all. She's already jumped on a couple of my veteran teachers. I pulled her in and said, "We're not having this. That is your team. You have a lot to learn, little lady." Now, I don't like those kinds of meetings, but I can do them if I have to. I'm not having something drive a wedge between our people and what we have that's been so good. That's been the love and trust of one another. (DR, 1/22)

Ms. Bowen acknowledged that such directive leadership was a challenge for Rippy because of her friendly, interpersonal nature. "I think she's willing to take on concerns ... It's a difficult thing to confront another professional who may be doing their best, and yet to tell them it's still not good enough" (HB, 1/25).

Effects on Teachers. As a result of Ms. Rippy's encouraging feedback, teachers reported a strong sense of responsibility toward their work as educators. Mr. Yeager said 
she inspired teachers to extend their understanding of their vocation beyond the classroom and school day:

She always reminds us that this [teaching] is a higher calling. That's the exact phrase she uses. It's a higher calling, that there are times you have to take on a whole lot as a teacher that other jobs sometimes don't require. It's not just a 7:30 to 3:30 job. Now, she's not really demanding about that. It comes across in the way she encourages you to take on extra things and try to help in ways that you can help out the school, where it's staying for Fall Festival or doing different things in your classroom with your teaching. As a teacher, I think she made me understand that this is a different type of job than just going and clocking in. (SY, 2/1)

In summary, for Research Question 1, data from Case Study B revealed that Donna Rippy used six theories of practice that accounted for her instructional leadership behaviors, including the following: (a) nurturing a positive learning climate, (b) inviting teacher input, (c) giving teachers autonomy, (d) encouraging teacher collaboration, (e) maintaining a focus on curriculum and student-centered instruction, and (f) providing feedback.

For Research Question 2, data indicated that teachers responded to Edmonds's leadership in the following ways: (a) teachers believed the school climate was positive (they shared a sense of "family" and "community", and that they were valued as human beings and professionals, (b) teachers were empowered and motivated, (c) teachers appreciated their freedom and autonomy, (d) teachers engaged in collaboration and teamwork, and (e) teachers had a sense of responsibility for student outcomes.

Finally, for Research Question 3, Donna Rippy, like Marie Edmonds in Case Study A, demonstrated reflection-in-action in that during her years as principal she had learned the importance of giving directive, corrective feedback when her preferred methods of modeling and using praise to encourage teacher effectiveness failed to work. 


\section{Case Study C}

Bill Kendall: Nurturing Others to Accomplish the Mission

Bill Kendall, principal of Homestead Elementary School (HES), was a 16-year veteran of education. A native of Eastern Kentucky, Kendall previously had worked seven years as a middle school industrial arts teacher in a city school district serving predominately poor and minority students. Kendall had been principal at HES for nine years at the time of this study. It was his first and only principalship. Kendall was well known in the district for his easy-going manner and warm personality. His theories of practice emerged primarily from his belief in the critical importance of establishing and maintaining positive personal relationships with staff and on his responsibility for carrying out the mission established by the district and state educational mandates.

\section{School Context}

Part of a county district of approximately 3,700 students, Homestead Elementary School served 427 students in kindergarten through eighth grade. Homestead was one of five K-8 community schools that fed into the one county high school of approximately 1,000 students. Sprawling, gently rolling farmland surrounded the school, a building constructed in 1998 that sits on the same site as other Homestead community schools dating back to the 1820 's, in the southern part of a geographically large, predominantly rural county. Homestead's staff of 36 teachers served students whose families primarily worked in the agriculture industry. While ethnically homogeneous (only $6 \%$ of students were minorities), more than half (57\%) were eligible for free or reduced lunch.

Many of Homestead's teachers were mid-career. Due to retirements and teachers whose spouses were transferred out of the community, Homestead had recently faced the 
need to hire a number of new teachers. Eight new teachers were in their first year at Homestead at the time of the study. Due to the school's remote, rural location, teacher recruitment was sometimes difficult. "Not a lot of people want to come to Homestead," Kendall said (BK, 12/11). “This is one of those places you've got to be headed there. You don't just pass through Homestead."

Despite the staffing turnover, however, the school had a long record of improving student achievement as measured by the Commonwealth Accountability Testing System (CATS), Kentucky's statewide student achievement program. By state policy, all schools must achieve an academic index of 100, indicating that almost all students are at proficient levels of performance in core subject areas, by 2014. The state measures schools' progress in two-year increments and Homestead's academic index rose from 69.4 in the biennium ending in 2002 to 84.8 in 2004 and 93.4 in 2006 . When Kendall became principal nine years ago, Homestead was the lowest-performing school in the district. Today, it is the highest-performing school. Kendall credited the school's positive culture as an explanation for Homestead's success: "I think our school has a very home-like atmosphere. The teachers really care about the kids and the kids know they are cared about. It's a good place to be" (BK, 12/11).

Katie Turner, a second-grade teacher at Homestead for 15 years, echoed Mr. Kendall's assessment that the school's achievement reflected the faculty's concern for student well-being. This concern was even "more important than learning:"

If we're not the ones who care about them, there's not going to be anybody. Until they know I care about them, it doesn't matter if I'm really good or I'm terrible. Sometimes you think, gosh, why can't they get it? When you're worried about where you're going to sleep tonight or what you're going to eat, or all this other stuff, learning to add and subtract is pretty minor. (KT, 1/8) 
Homestead's school climate reflected Kendall's emphasis on positive relationships. Teachers shared a strong sense of responsibility toward meeting the mandates established by the district and state, reflecting Kendall's commitment to faithfully carrying out the school's mission.

\section{Bill Kendall: Research Findings}

Five theories of practice described Mr. Kendall's instructional leadership. Each theory of practice is described below, including the core assumptions that made up the foundation of the theory of practice, the action strategies that logically emerged from those assumptions (Research Question 1), and the effects of the theory of practice on teachers (Research Question 2). When data suggested that Kendall had engaged in reflective practice by adjusting or modifying his theories of practice by revising his assumptions or action strategies, this process is also described (Research Question 3). Theory of Practice 1: Building Positive Relationships with and among Teachers

Building strong personal relationships with and among teachers was a key theory of practice for Mr. Kendall. This theory of practice emerged from his assumption that making teachers feel valued and affirmed and sharing a sense of community enhanced teacher effectiveness. Kendall also assumed that positive teacher relationships were the template for building positive student relationships, which in turn would lead to academic success. Teachers described a variety of strategies Kendall used to nurture positive teacher relationships and how these strategies made them feel valued and affirmed (see Figure 18).

Assumptions. Mr. Kendall assumed that demonstrating care and concern for teachers encouraged teacher motivation and effectiveness: 


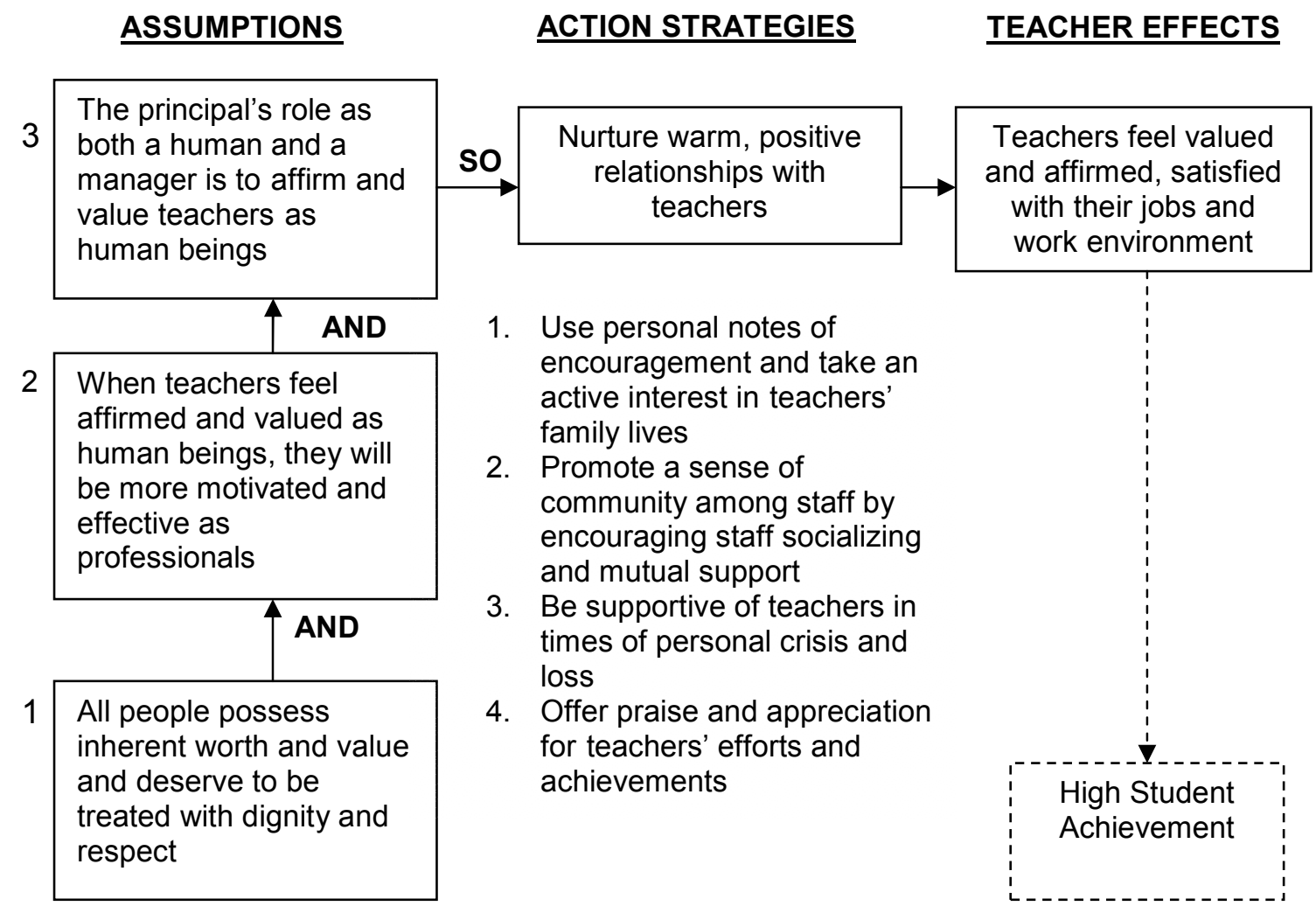

Figure 18. Bill Kendall, Theory of Practice 1, for nurturing positive relationships, including assumptions, action strategies and teacher effects. A tacit assumption of all instructional leadership theories of practice is that these action strategies will indirectly result in higher student achievement, though this link is not explored in this study (indicated by dashed lines in the figure).

I try to let them know they are cared about as people, not just someone filling a position in my building. If I think my boss cares about me and the circumstances I'm in, I'm going to do my best to please him. I feel like my staff do the same with me. There is a relationship that has to be cultivated and it has to be based on care and respect. I think that's one of the main things I try to do. (BK, 12/11)

Teacher participants repeatedly used the words "encourage," "encouraging," and "supporter" in reference to Kendall's role and his relationship with staff (BK, 12/11; BK, 1/8; BK, 1/9; KT, 1/8; SG, 1/8; CG, 1/15). The chief quality identified by teachers in Kendall's leadership was his caring, compassionate concern for others, and they 
understood this to be the source of many of his instructional leadership behaviors. A 17year veteran who taught kindergarten at Homestead, Sherry Gann stated, "He's a caring person. You can really see his compassion. He focuses on the human side of everything" (SG, 1/8). Carrie Gergan, who started working at Homestead the same year Kendall became principal and served as the school's guidance counselor, agreed. "He's a compassionate person. If something goes on with us personally and he knows about it, he's very kind. I know that he believes that before people care about what you are doing, they have to know they are cared about" (CG, 1/15).

Ms. Turner emphasized that Kendall's concern for the well-being of others was his core belief. "Mr. Kendall cares about his teachers as people. I know he cares about Katie Turner the person, probably more than he cares about the job that Katie Turner the teacher is going to do" $(\mathrm{KT}, 1 / 8)$.

Similarly, teacher participants were unanimous in their conviction that he possessed a core assumption that each child, regardless of their economic circumstances, has a right to a quality education, enhanced by strong personal relationships with teachers (KT, 1/8; SG, 1/8; CG, 1/15).

Gann confirmed that Kendall's dedication to the "underdog" children was a hallmark of his leadership:

He highly believes that every child, whether they are a poor or rich, whether they come from the projects or the best homes in the county, has the right to learn and we should treat them equally. He really stresses that and shows empathy for those children. We might not know what [backgrounds] they came to school with. He'll say, "We really need to think about these things. We don't know what that child encountered [before coming to school]. There may be drug use going on, there may be guns in the home. So before we jump to conclusions - that they're here to learn this, this, and this - we first have to care about that child, about what they are feeling. (SG, 1/8) 
Kendall credited his experiences teaching inner-city youth and his reading and study of Ruby Payne's book, A Framework for Understanding Poverty (2003), as sources for this assumption (he later required his whole staff to read and discuss Payne's book). This assumption was also rooted in his experiences growing up in a largely-poor, rural community in Appalachia. "In my home county I was lucky enough that my family had more things than most people. ... I always had a great deal of compassion for those friends of mine who didn't have the opportunities I had" (BK, 1/16). These experiences shaped his assumption that schools should provide a high-quality education for all students, especially the poor:

That should be the goal of every educator because the key to unlocking the future for any child, especially a child who is from a lower socioeconomic level, is education.... The only way we're going to change the cycle that these kids live in and that affects our world and community is to educate them all. Some of these kids aren't going to have an advocate ... so I push to help them because someone has to reach out a helping hand and pull them up from where they are to better themselves. (BK, 1/16)

Action Strategies. Teachers pointed to Mr. Kendall's use of personal notes of encouragement, and his practice of visiting teachers, students and their family members at the hospital and funeral home as examples of how he nurtured strong relationships in the hopes of supporting school success (KT, 1/8; SG, 1/8; CG, 1/15). These efforts did not necessarily relate directly to instructional issues, but Kendall believed that helping staff feel good about themselves, their work, and their relationship with the principal, would enhance their instructional efforts.

A key part of this strategy included supporting teachers in issues related to their family or personal lives:

If you've got a sick child, a sick parent, a sick spouse, your family comes first. Some people don't believe that. Some principals honestly think that if a teacher 
has a sick child, their duty is still to come into work and do their best. I don't buy that. You're not going to be giving all of yourself if your mind is somewhere else worrying about someone you care about. Your duty is first to your family. Then, when they are all right, you worry about coming to school. I don't give my teachers a hard time. That's one of the main things I try to do. If I think that my boss cares about me and the circumstances I'm in, I'm going to do my best to please him. (BK, 12/11)

Kendall routinely visited teachers or their family members when they were in the hospital, or visited the funeral home when a teacher's family member passed away. Ms. Turner related personal examples. "He's sat at the hospital and cried with us [when her father died]. He's celebrated the birth of both of my children. He's been to the hospital to sit and cry with a parent of a student who died [in a sports-related accident]. We've been all those places" (KT, 1/8).

Kendall's efforts to foster strong personal relationships extended to include building a sense of community among the staff. Prior to his arrival as principal, the staff was largely fragmented, especially in grade-level divisions between primary and middle grades teachers. Personal relationships were often strained:

In these K-8 buildings you've got the prime example of how you can work together to make sure you're filling in any [academic] gaps and you're not wasting time. But when you've got teachers who aren't speaking to one another, it creates a little problem. The first year I was here, I recognized right off that I had two buildings under one roof and my first goal was to mesh us together and put us all on the same page, all with a common goal. And that was not easy. (BK, 12/11)

Kendall's primary means of promoting stronger staff relationships was to sponsor and encourage social events both during and outside of the school day, including ice crime socials and annual back-to-school, Christmas, and end-of-school parties for faculty and their families, which he hosted at his home. Summer staff retreats allowed time for 
relationship building (BK, 12/11; CG, 1/15; SG, 1/8). These efforts contributed to a stronger sense of community and common purpose among the staff:

If you build those relationships and friendships you are going to work better with someone you like and get along with, someone you consider a friend. You're going to do everything you can to help them and hold them up at times when they're not able to stand on their own for whatever reason. And that has helped tremendously in building that feeling of community and family. (BK, 12/11)

Kendall became visibly emotional when discussing his affection for the teachers, and the importance of his relationship with the staff to his leadership. "I love this staff," he said, choking back tears. “There's nobody on this staff I wouldn't do anything for. They are mine. I want to love them, protect them, do anything for them. I hope they know that" (BK, 1/9).

Effects on Teachers. As a result of Mr. Kendall's emphasis on personal relationships, teachers felt valued and affirmed and desired to remain a part of the Homestead community. Teachers shared Kendall's commitment to promoting individual student success. Ms. Turner endured a long commute from her home to work at Homestead because of the positive school culture Kendall helped create: "I drive almost 45 minutes a day to be here because I love being here. There's several other schools much closer to my home and I have friends and family who think, 'Can you not get a job there?' But I don't want to leave.” Ms. Turner also valued being at Homestead because she wanted that school environment for her own daughter. "We're in a school where she's loved and she's cared about and she knows that. And that's how we feel as teachers, and that leads us to work even harder" $(\mathrm{KT}, 1 / 8)$.

Kendall affirmed that the teachers also shared a sense of community as a staff (BK, 12/11). 
I don't know anybody in the building who doesn't get along. There are no groups that don't get along with other groups. We all seem to have a common goal. We all want what's best for each grade level. We all realize that how well one group does greatly affects how well the entire school does. ... Everybody who comes here sees that. If we have [accreditation team] visitors or scholastic audits, one of the things they always say is that we are just a big family. (BK, 12/11)

Ms. Gann agreed, and noted that a deep concern for the emotional well-being of students was the heart of the faculty's vision for the school:

Everybody's got that common thread, the common goal for these kids, from preschool to eighth grade, that we meet their needs in the most warm and caring environment that we can and that we first and foremost think of their heart. We think of their heart first, because we can't reach them if they don't know we care. That's what everybody here believes. (SG, 1/8)

To summarize Theory of Practice 1, Mr. Kendall assumed that, because all people possessed inherent dignity and worth, if he as principal affirmed and cared for the personal needs of teachers, they would experience higher levels of motivation and job satisfaction. Kendall translated these assumptions into action strategies, including using personal notes of encouragement and praise, promoting a sense of community by encouraging staff to socialize, and supporting teachers in times of personal crisis. Teachers reported key effects of this theory of practice, including feelings of being valued and affirmed and satisfaction with their jobs and working environment.

\section{Theory of Practice 2: Unifying Teachers to Implement District and State Directives}

Mr. Kendall assumed his role as principal involved unifying his staff to work toward a common purpose, defined by a commitment to increase student achievement, and by district and state mandates. He used interpersonal skills to motivate teachers and unite them, even when he had reservations about some mandates imposed from outside the school. Teachers responded to this theory of practice by faithfully endeavoring to 
meet the goals and accomplish district and state initiatives, in large part because of

Kendall's affirming, relational approach (see Figure 19).

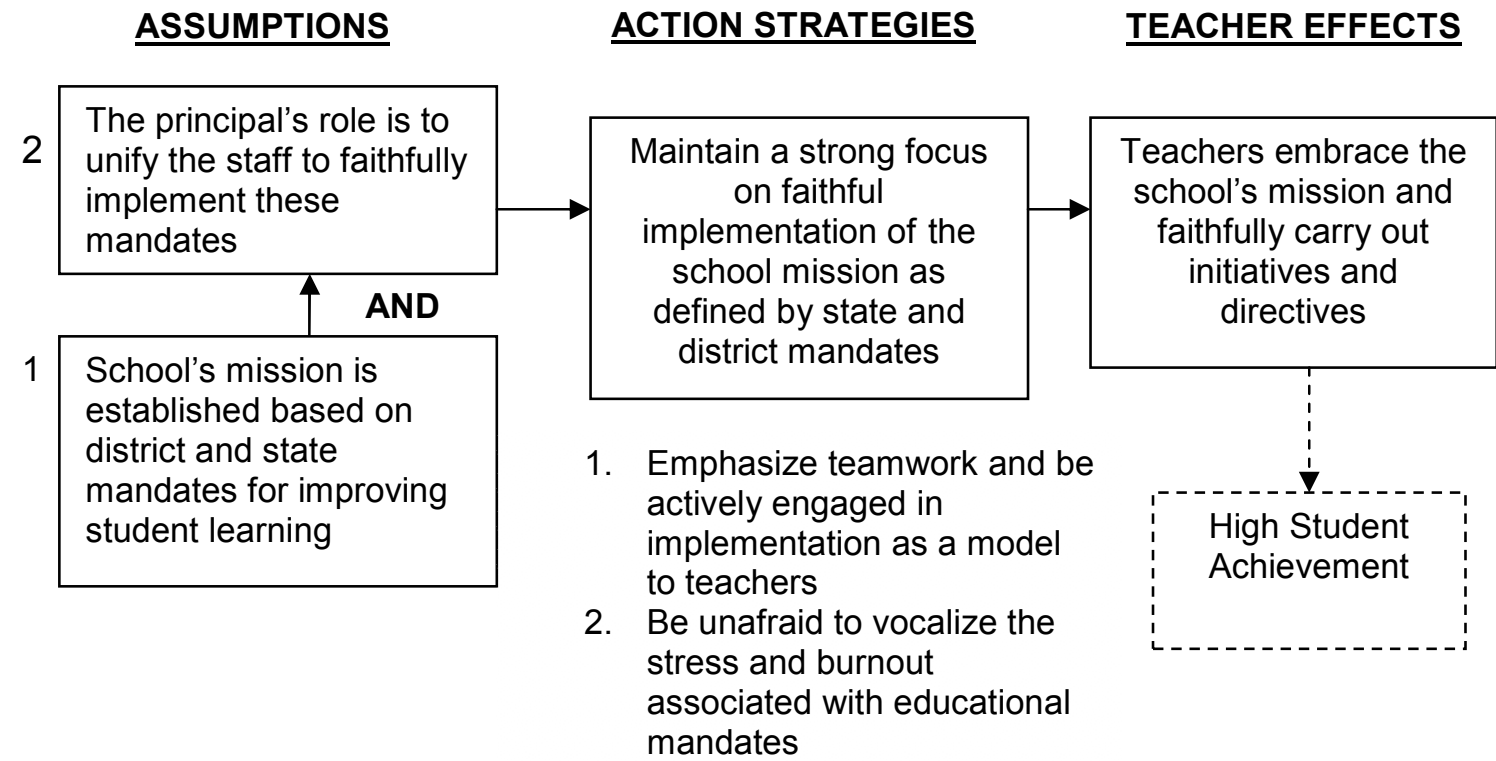

Figure 19. Bill Kendall, Theory of Practice 2, for unifying staff around a common mission, including assumptions, action strategies and teacher effects. A tacit assumption of all instructional leadership theories of practice is that these action strategies will indirectly result in higher student achievement, though this link is not explored in this study (indicated by dashed lines in the figure).

Assumptions. Mr. Kendall assumed his role was to "keep them [the teachers] pointed in the right direction, and the one who sees to it that they all end up doing what needs to be done in the way it's supposed to be done" (BK, 12/11). He defined the "right direction" and "what needs to be done" in part as achieving the state-mandated goal of having all students perform at proficient levels in all subject areas by the year 2014, but included preparing students for life-long learning and maintaining a positive school culture as part of the school's mission as well. Improving test scores is "the big star we've got to head toward. But between here and there, to me, there's an even more important thing, and that is instilling in the kids a love of learning" (BK, 1/9). 
Homestead's school district was well known in the region for its strong central office. Mandates for implementing new instructional programs to improve student achievement were common, and Kendall saw part of his role as dutifully carrying out these district directives and making them a part of the school's core mission. The leadership structure of the schools in the district reinforced this role. Each school, including Homestead, employed a curriculum specialist, responsible for learning new instructional techniques (including those mandated by the district) and teaching them to the rest of the staff. 'I'm not the one who's out there training teachers, but I'm the one who says, 'This is what we're going to do, this is the expectation, these are things we have to accomplish"” (BK, 12/11).

Like his commitment to the underprivileged, Kendall credited his commitment to implement district mandates to his family background and personal experiences:

I've always been that way. That comes from childhood too. If you're told to do something, you're going to do it. That was the expectation in my home. It's the expectation I've put upon myself my entire life. If somebody tells me to do something, I'm going to do it, but I'm going to do it in a positive way. I'm not going to grumble and groan about it. I might say, "I know we might not want to do this, but we're going to do it." (BK, 1/9)

Kendall tried to capitalize on the strong sense of community among staff to effectively unify the faculty around the school's core mission. "Alone you have the strength of one, but together you have the strength of many. It takes everyone working in unison to cover all the standards and make sure we have no gaps in curriculum. If the right hand knows what the left hand is doing you are less likely to have a problem" (BK, $3 / 24)$.

Action Strategies. Mr. Kendall tried to capitalize on the staff's sense of community to rally teachers around implementing the school mission, which he defined 
as improving student achievement, attending to the academic and emotional needs of the individual child, and implementing the various instructional programs mandated by the district (BK, 12/11, 1/9).

Teachers believed the positive interpersonal relationships Kendall built with and among teachers helped unify the staff in these efforts, even when teachers were sometimes reluctant. "He's willing to do what is instructed of him, or what is given to him as a directive," Ms. Gann explained:

He tries to follow the guidelines that he's given from the central office, even though it might come down and he knows we feel pressure. He feels pressure too. ... Even though he's going to step on some people's toes, and it may make him feel bad, he's going to pursue it and he's going to expect it. We're all in this together, so we're just going to try and do the best we can. (SG, 1/8)

Kendall tried to support district initiatives by participating in required training programs alongside teachers:

I'll go. I'll sit right there with them through the whole thing, even though it might not really affect me. I'm probably not going to use a lot of the things they are going to learn that day, but I'll sit there with you. Because if it's going to take up your precious time, then I'm not going to ask them to do anything I'm not willing to do. $(\mathrm{BK}, 1 / 9)$

Ms. Gann indicated that while Mr. Kendall supported all district initiatives, he also tried to protect teachers from overload by affirming the stress they faced and suggesting that they implement programs gradually:

To have so many things put on a faculty at one time ... he understands, and says, "Try to do a few and then next year try to do some more. You can't do it all at once. ... Don't try to do them all if it's going to make everything become confusing." Him admitting that makes us feel like he's a person and doesn't expect us to incorporate everything all at once. He expects the best of us and wants us to do the very best we can, but just a little bit at a time. (SG, 1/8).

Ms. Turner agreed: "He tries to protect us based on what is most important to our vision and keep the district happy too. .. . There have been times when we've had our hands in 
so many different things that you can't do anything well, and we let go of some things and focused and got some calm back around us" (KT, 1/8).

Kendall emphasized that he was not compromising in fully implementing district directives, but admitted that he encouraged a gradual approach: "It's not that we're not going to do the others [directives that receive less attention], but we're going to pick the ones that are going to make the most difference for us, make the most benefit to us, and focus more energy on those" (BK, 1/9).

While Kendall sometimes found the district mandates burdensome, he believed they were always in the school's best interest:

I always tell the teachers, we've never been asked to do anything that has not bettered us. Everything we've ever been asked to do or told to do-some things we wanted to do and some things we didn't—nothing has ever harmed us. They've always bettered us in some way. (BK, 1/9)

Kendall was more straightforward in asking teachers to implement district-wide directives, whereas he once was more concerned with teachers' feelings, according to Ms. Gann: "I feel like he just tells it like it is, but maybe then [when he was lessexperienced], he might say, 'This is how it is and sorry.' But now he might say, "You know, everybody has to pull their weight and this may not be something you want to do this year, but we'll have to do it next year and we can be mad about it or upset but that's just the way it is" (SG, 1/8). Ms. Gergan agreed: "Where he may have been a little more apprehensive to be blunt or forward when he began, he's not now. He'll say, 'This is it.' But he's not mean about it" (CG, 1/15).

According to Gergan, Kendall's effectiveness was enhanced because faculty had grown to respect his opinions and knew he cared about them. "He has credibility now," she explained. "It's kind of like your parents. You know they care about you and love 
you, but there are just some things you have to do sometimes. It's just like a parent correcting a child. He has a big heart. He knows he has to step on toes sometimes, but he knows a good way to do it" (CG, 1/15).

Ms. Turner attributed Kendall's increased directness less to any changes in his underlying assumptions about instructional leadership and more to his growing knowledge of elementary education:

He's really emerged as an instructional leader. His background, he was an industrial arts teacher. He didn't know a lot about a primary classroom. That was totally foreign to him. He's gone to the trainings and sat there with us and knows more instructionally. There was a time he would have never sent out a group of articles to read and sat down and had conversation with us because he wasn't as instructionally focused as he is now. (KT, 1/8)

Effects on Teachers. Teachers at Homestead shared a strong sense of community

and embraced Mr. Kendall's commitment to carrying out district directives. "Every now and then he might have to drag us kicking and screaming, but most of the time we go where we're led or where we're told we are supposed to go," according to Ms. Turner (KT, 1/8). Because teachers trusted Kendall, they were more willing to follow directives:

When he's telling us one of those things that we have to go to kicking and screaming, or that we really don't want to do, we know it is either, one, because that's what he really believes we need to do and that's what's best for our kids and our school, or two, it's one of those things that he has no choice about and we have to do because it's a "have to," so suck it up and go on. It's not about a show, it's not about being better than anybody else, it's about doing what's best for our kids. (KT, 1/8)

The sense of community and unity contributed to a shared sense of school mission, according to Turner:

We've created a shared vision of where we want our school to go, and he's trying to make sure we have the resources, the materials and to protect that vision ... For whatever the reason, we're a very self-motivated group of people who want to succeed ... we're really proud of where we've come from and where we want to 
go with our kids and caring about kids. Honestly, if you don't share that vision, you probably don't stay around a real long time. $(\mathrm{KT}, 1 / 8)$

Bill Kendall's second theory of practice emerged from a deeply held assumption that as principal he was responsible for faithfully implementing the mandates and mission established for the school by the district and state. He capitalized on the faculty's sense of unity and community to maintain their focus on carrying out the school mission, and participated in implementation alongside teachers as a model for them. Kendall was unafraid to vocalize his own stress and sense of burden related to these mandates, but held a positive attitude toward the benefits of implementation, and encouraged a gradual approach. Teachers appreciated Kendall's directness and embraced their responsibility toward implementing mandates and carrying out the school's mission.

\section{Theory of Practice 3: Providing Feedback}

Based on an assumption that to improve performance, teachers needed ongoing, positive feedback, Mr. Kendall intentionally made efforts to give teachers specific encouragement, advice, and support related to their teaching. In cases where his preferred method of using positive feedback failed to address problems with a teacher's performance, he used more corrective forms of feedback, including improvement plans and non-renewing un-tenured teachers. Teachers felt appreciated and affirmed by Kendall's efforts to provide feedback, and had confidence that even his corrective forms of feedback were appropriate and just (see Figure 20).

Assumptions. Mr. Kendall assumed feedback helped keep the teachers focused on the mission:

It's my responsibility to make sure that good instruction is going on in the building, that if I've got someone who is the weak link, it's my responsibility to strengthen them, provide them with the PD [professional development] they need 


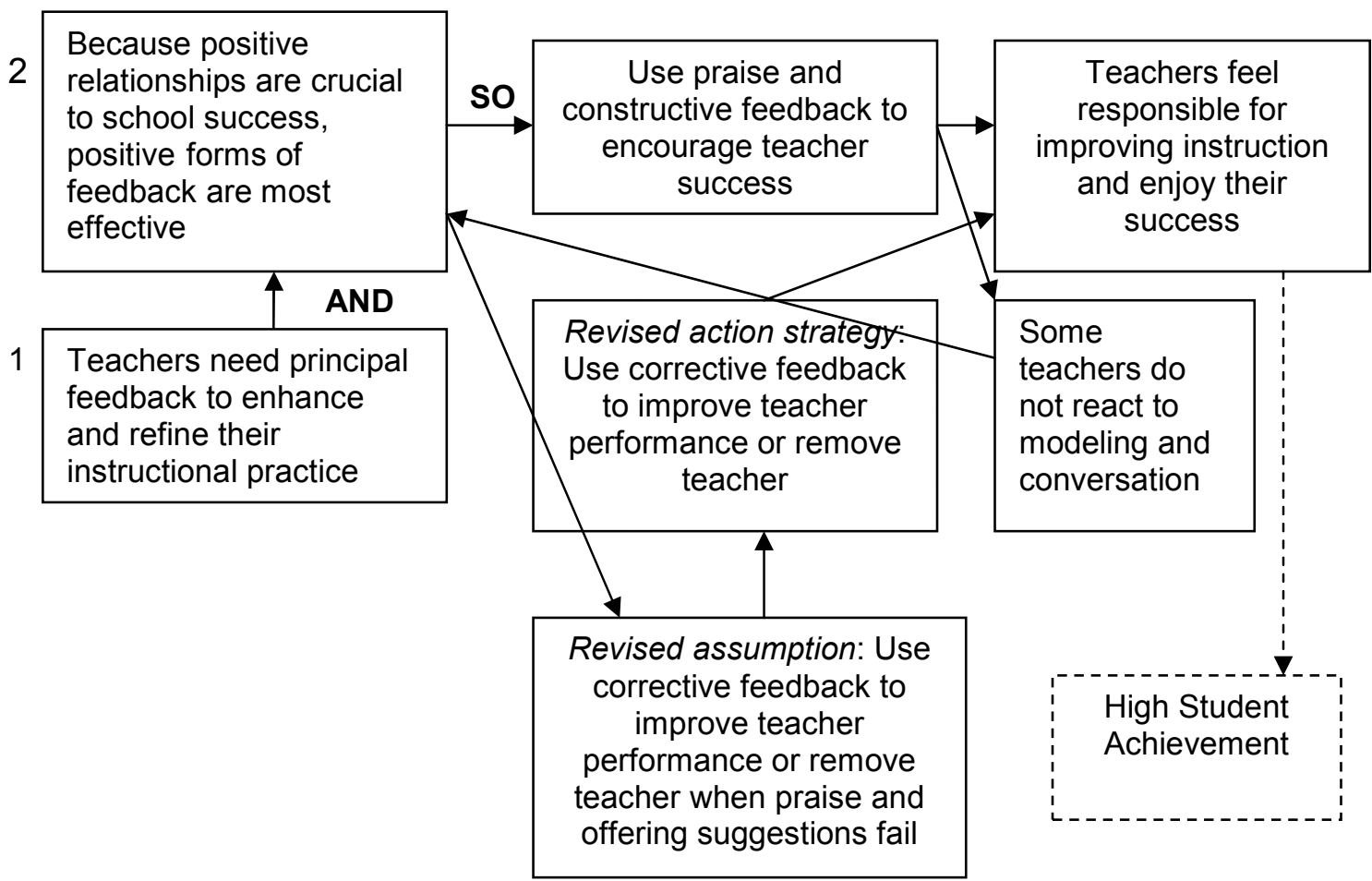

Figure 20. Bill Kendall, Theory of Practice 3, for providing feedback, including assumptions, action strategies and teacher effects. A tacit assumption of all instructional leadership theories of practice is that these action strategies will indirectly result in higher student achievement, though this link is not explored in this study (indicated by dashed lines in the figure).

to become a better teacher, or if need be, the person who invites them to leave. Ultimately it all rests on my shoulders, and that can be a little overwhelming. (BK, 12/11)

Kendall's feedback reflected his assumption about the importance of strong interpersonal relationships: "They need feedback, both positive and constructive. They want to know that you are paying attention to what they are doing" (BK, 12/11). Kendall's feedback usually took the form of supportive notes or e-mails, reviews of teacher lesson plans, formal observations, informal walkthroughs, and pairing struggling teachers with mentors (BK, 12/11). 
Sometimes he used corrective feedback, such as putting teachers on improvement plans or asking them to leave, reflecting his belief that he should only retain teachers who were effective: "You're not doing anybody a favor by keeping them in a job where they're going to be miserable and make your life miserable. You're not helping them or yourself and you're certainly not helping the kids" (BK, 12/11).

Action Strategies. Mr. Kendall's preferred method of feedback was providing encouraging notes and e-mails praising teachers for their efforts:

If I'm in a classroom, and I've seen a lesson that is really, really good, within the next day or so I want to let them know, whether it's in person or e-mail or a note or written on the observation, what I liked, what I thought could have been better, how they might have enhanced it, or if they totally missed the mark, that too. (BK, 12/11)

"He's good about giving pats on the back, little notes and stuff like that," Ms.

Gergan explained (CG, 1/15). Ms. Turner had a note Kendall gave her two months before still tacked above her desk:

Katie, where do I start? I could write a book about all you do to help your students, peers, and our school! I appreciate you more than I can put into words. Your giving nature never ceases to amaze me. I am so lucky to call you my friend. Thank you for being you! (Note observed in teacher's classroom, 1/8)

Kendall also used "walkthroughs," brief, informal classroom visits, to provide feedback to teachers (BK, 12/11). "He walks through and I know he stops at my door, probably daily, but I'm busy and I don't always see him. He might pop in and walk right back out, but you know he's there" (SG, 1/8). During an observation, Kendall reviewed a walkthrough checklist he had created to document his classroom visits and noted the presence of certain "look for's," strategies that he expected to see all the teachers using (Observation, 1/8). He shared results of these walkthroughs with teachers on a periodic 
basis and reviewed lesson plans the teachers submitted to him on a weekly basis (Observation, 1/8).

Despite his deep personal affection for teachers, Kendall used constructive feedback to assist teachers who were struggling to be successful, including placing them on corrective action plans and providing other supports for improvement. "Inevitably, I'm the one who goes in there and evaluates them on whether they are doing the things they are supposed to be doing and making the gains and the strides we expect them to make," he explained (BK, 12/11). He invited other administrators from the district to observe struggling teachers, and often paired them with a more experienced teacher for support (BK, 12/11; KT, 1/8). A teacher who he once placed on a corrective action plan was still teaching in the building: "She turned things around and instruction improved greatly. I have no problems with the instruction that takes place in her classroom today" (BK, 1/15).

In other cases however, the teachers did not improve and he eventually "invited them to leave" (BK, 12/11). This occurred once when he decided to non-renew an untenured teacher, and on two other occasions when the threat of a corrective action plan and his verbal encouragement induced teachers to retire early or leave for other jobs (BK, $12 / 8,12 / 9)$. He emphasized "it was not easy" because he cared about the teachers as people.

If they are not being successful and I've done everything that I can to help them, I'm the one who then invites them to leave.... I told a teacher once, the absolute best thing you can do for your career is bring me a resignation tomorrow. That's not easy when you've got a teacher sitting here and it's her first year teaching and tears are rolling and she's paid all this money to go to college and get a job as a teacher because she thinks that's what she wanted to do. (BK, 12/11) 
Teacher participants noted that even Kendall's corrective feedback was typically compassionate and supportive. Ms. Gann acknowledged Kendall was willing to ask weak teachers to leave, but affirmed that he only did so after working hard to help the teacher improve, and that the decision always came with a personal emotional cost for him:

He doesn't want to hurt anybody's feelings, but yet, he does make it clear what is expected and tries to do it in a caring way. I've never seen him really go off on anybody ... he does it in a way that pulls people back in.... I do know that teachers have come and gone [at his request], but I have never heard of it being in an unfriendly way or a way that someone is just booted out. I feel like he worked with them. They had an opportunity. He had them work with mentors. (SG, 1/8)

Ms. Turner agreed that Kendall's feedback, even when corrective, usually came with a sensitivity to the individual teacher. "When he finds a situation that he doesn't see as ideal ... he's going to try to find a way to support that teacher and provide help and assistance rather than just write you off and get rid of you:"

I've been involved with him identifying the problem. He'll say to me, "Here's somebody, I want you to work with this teacher, and see what we can do [to help them]." He's going to do that before he's going to say, "Okay, you have to leave." But ultimately, he thinks it's his job to make sure we have an environment where learning can take place. (KT, 1/8)

Kendall agreed that he had become more directive over the years while simultaneously inviting more teacher input in decisions. His confidence about leading elementary instruction had increased (BK, 1/16). In the dialectical exercise in which he recounted a post-observation conference of a lesson that did not go well, Kendall described a 23-year veteran middle school teacher who lectured throughout an entire class period, oblivious that students were sleeping or not paying attention. In his recollection of the story, Kendall's conversation focused on the teacher's inattentiveness to the students' response and ways to correct the problem: 
P: You have a great deal of potential to be a wonderful teacher, but ... I was most disappointed [with your performance today]. You were unaware of things going on right in front of you. Not one time during your class did you stop lecturing or get from behind your podium. Is this how your class normally functions?

T: Pretty much. I do a lot of lecturing. But I did not notice anyone sleeping. P: That's the problem. You were so into your lecture that you tuned out the lack of engagement of the students. Have you ever thought about cooperative grouping or using some hands on activities?

T: Yes, but I do not find them very productive ...

P: Okay, we have lots to work on, so I am going to ask you to correct the things we have spoken about and within two weeks, I am going to do another observation to see if you are making progress. (BK, 1/15)

In the column in which he related the thoughts and feelings he was experiencing during the post-observation conference, Kendall described his shock that the teacher was so unaware of how his instruction was affecting students. "It just blew my mind that someone could spend 45 minutes lecturing and never take note of the students they were teaching," he wrote. "I wish I had videoed him so that he could watch the class and how they were reacting or not reacting to him" $(\mathrm{BK}, 1 / 15)$.

The teacher did not make the recommended corrections, but did retire early at the end of the school year, buying out his last four years of employment. While he believed that the teacher left because of his threats to put him on a corrective action plan, Kendall still wondered if he should have been more directive:

If I had to deal with him today, I would probably have been a lot more forceful with him from the beginning instead of giving him time to shape up. I just kept hoping he was going to improve, but he never did. I would've been more directive and forceful in that this is my expectation and you will meet it. (BK, $1 / 15)$

Because of incidents like this, Kendall related that he indeed became more directive over the years. Sometimes he made forceful decisions deliberately to counteract his reputation for being focused on the emotions of others. "I am a very heart-felt person 
who lets their heart lead them, and sometimes you give the impression you are weak," he shared. "Sometimes you have to step on toes, even of people you really respect, so you don't lose credibility. It's not always intentional; it's a necessary evil" (BK, 1/15).

Effects on Teachers. Teachers "go above and beyond what is asked of them most days" in their efforts to help students, according to Mr. Kendall (BK, 12/11). Ms. Gergan said Kendall's leadership contributed to teacher desires to work hard. "He has high expectations," she reported. "The teachers know they are accountable. They want to do what they are supposed to do and impress him, have good test scores and for the students to do well" (CG, 1/8).

To summarize Kendall's third theory of practice, he assumed teachers needed feedback to refine and improve their practice, and because he placed such a premium on maintaining positive personal relationships, Kendall used action strategies such as giving constructive feedback and using praise to encourage teacher success. Most teachers responded to these efforts by internalizing responsibility for on-going professional improvement. However, Kendall found that some teachers did not respond to this interpersonal approach, and like Marie Edmonds in Case Study A, Kendall revised his assumptions to deal with such teachers by providing more directive, corrective feedback. Theory of Practice 4: Encouraging Teacher Professional Growth

Mr. Kendall believed that educators should strive to improve their skills by studying new research, learning new techniques, and especially by collaboratively reflecting on new information relevant to their students. He fostered this type of professional growth by regularly sharing research and instructional ideas with teachers and encouraging them to explore the concepts together, and by providing opportunities 
for on-going teacher professional development. Teachers responded to this theory of practice by actively embracing new instructional approaches and accepting responsibility for continual improvement (see Figure 21).

ASSUMPTIONS

For principals to

2 effectively, they must be actively engaged in the professional growth process "We as teachers must be continuously improving our craft. Students change and we must change to meet their needs"
ACTION STRATEGIES

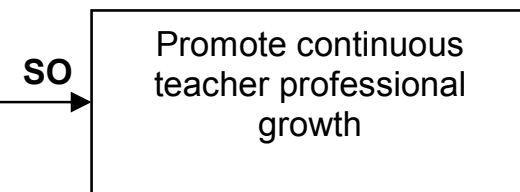

Examples:

1. Promote professional development opportunities

2. Share articles on best practices and new research

3. Participate in PD alongside teachers
TEACHER EFFECTS

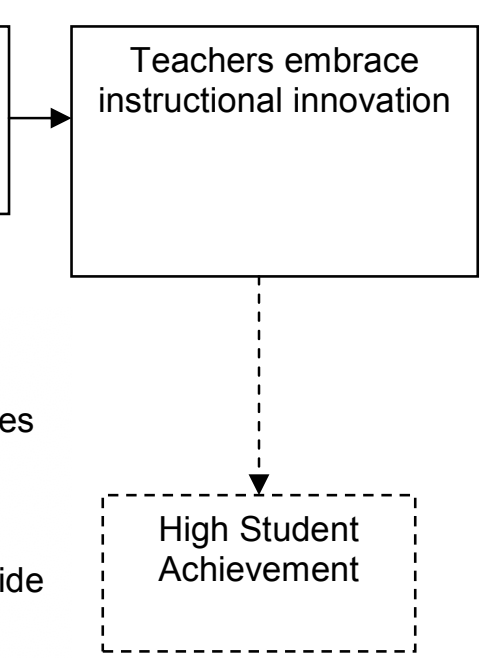

Figure 21. Bill Kendall, Theory of Practice 4, for encouraging continual professional growth, including assumptions, action strategies and teacher effects. A tacit assumption of all instructional leadership theories of practice is that these action strategies will indirectly result in higher student achievement, though this link is not explored in this study (indicated by dashed lines in the figure).

Assumptions: Mr. Kendall assumed that the challenges of educating today's

students were so great that teachers must keep continuously learning new skills. In this

way, teachers and students were similar:

We want students to be life-long learners and we must model this. You never stop learning until you hit the grave. ... We as teachers must constantly be working and improving our craft. Students change, programs change, ideas change, and we must change to fit the need. Professional growth is a must to keep up with what kids need beyond their basic need of love and understanding. (BK, $3 / 24)$

Action Strategies. Ms. Gann said Kendall was supportive of professional development and the district's summer academies for ongoing professional growth. "I can't remember a time we've gone to him to ask to go to conferences and he hasn't come 
through for us with support and money to go" (SG, 1/8). Kendall liked to individualize professional development for the needs of particular teachers or groups of teachers:

So that's why, as I read articles in Educational Leadership or Education Week, or any other book or magazine I pick up, if I think this article or book is really good, and it will pertain well to teachers $\mathrm{X}, \mathrm{Y}$, and $\mathrm{Z}$, then I'll copy it and give it to specific teachers. If it's something related to teaching math with music, I'll send that out to my math teachers, for example. (BK, 12/11)

Kendall's most common technique for promoting ongoing teacher learning was to share professional articles and then ask teachers to read and discuss the articles in teacher teams. Ms. Turner described how Kendall often attended these meetings himself and participated in discussion, or asked the team leader to share notes from the discussion (KT, 1/8; BK, 1/9). The researcher observed Kendall providing a book on research-based instructional practices to a new teacher, and reading an article on Attention Deficient Disorder which he planned to share with teacher teams (BK observation, 1/8). Such tactics "open up dialogue among those teachers" about important instructional topics they might not otherwise talk about, and forced them to engage in study and discussion (BK, $1 / 8)$.

"I give my teachers articles to read on the latest research findings. I pass out books to read. That's almost a weekly thing" (BK, 12/11). During observations, Kendall provided a book on research-based instructional strategies to a new teacher and made copies of an article on teaching children with Attention Deficit Disorder (Observation, 1/8). He noted that sometimes he asked the entire staff to read the same book, such as Ruby Payne's Framework for Understanding Poverty (2003), or Marzano, Pickering, and Pollock's Classroom Instruction That Works (2001) (BK, 1/9). 
Teachers noted that Mr. Kendall often met with teacher teams to discuss articles (SG, 1/8; KT, 1/8; CG, 1/15). "He puts himself out there and takes the time to be with us and go over it," Ms. Gann reported. "That's his time as well as ours" (SG, 1/8). Kendall said that when he attends meetings he tries "to be quiet and listen. The discussion is really good because it opens up dialogue among those teachers on topics they might not otherwise even talk about" (BK, 12/11).

In addition to article and book studies, teachers said Kendall encouraged them to attend professional development opportunities and summer academies (SG, 1/8; KT, 1/8).

Effects on Teachers. Teachers at Homestead embraced the concept of continual professional improvement and appreciated Kendall's efforts to foster their growth (KT, 1/8; SG, 1/8). "Teachers here want to keep learning and getting better," Katie Turner reported. "Mr. Kendall gives us opportunities to keep growing, and while it's timeconsuming, I especially like the chance to learn alongside other teachers. I get so much more out of an article or PD activity when I can share it with others" (KT, 1/8). Theory of Practice 5: Encouraging Autonomy and Input

Based on an assumption that teachers have more relevant knowledge about instructional practice and student needs than he did, Mr. Kendall intentionally encouraged teacher autonomy and input into decision making. Teachers shared a strong sense of ownership for the direction and mission of the school as a result (see Figure 22).

Assumptions. Mr. Kendall relied on teachers to share their knowledge to make effective decisions as principal. He believed that as professionals, he should give teachers broad leeway in meeting their students needs, and respected the expertise and 
ASSUMPTIONS

$2 \begin{aligned} & \text { Good teachers know } \\ & \text { how to improve student } \\ & \text { achievement }\end{aligned}$

\section{AND}

1

The principal does not have all the answers for increasing student achievement
ACTION STRATEGIES

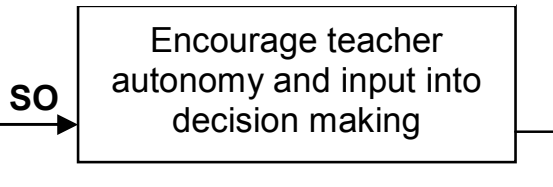

1. Encourage teachers to experiment in their classrooms

2. Listen to teacher ideas and input

3. Solicit teacher feedback

\section{TEACHER EFFECTS}

\section{Teachers experience a} sense of freedom and appreciate their work autonomy

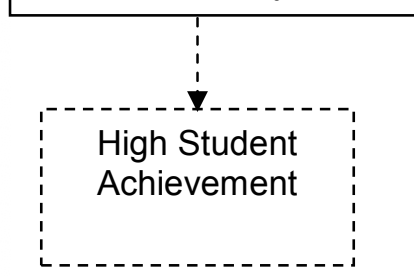

Figure 22. Bill Kendall, Theory of Practice 5, for encouraging teacher autonomy and inviting teacher input, including assumptions, action strategies and teacher effects. A tacit assumption of all instructional leadership theories of practice is that these action strategies will indirectly result in higher student achievement, though this link is not explored in this study (indicated by dashed lines in the figure).

insights of those who worked with children on a daily basis. His comments suggested that when teachers experience autonomy and are invited to share in decision-making, they experienced higher levels of job satisfaction and effectiveness:

Teachers need to know that their opinions matter. They too have a stake in this and their input is most valuable. Collectively, they have hundreds of years of experience to bring to the table. We need to tap into this source of knowledge. Don't reinvent the wheel; learn from each other. If you have an interest in something, a stake in something, you are going to want it to be successful. (BK, $3 / 24)$

Action Strategies. Kendall prided himself on supporting his effective teachers by giving them freedom and involving them in decision-making processes: "I've got a wonderful staff, very hard working people. I don't have to stand over them and crack a whip, look over their shoulder" (BK, 12/11):

I have faith in the teachers. They appreciate that I don't think they are ignorant or unable to perform. I don't breath down their necks. I don't dictate. I hired them and put them in the position because I believed in them. As long as they are performing well, students are doing well, what more can I ask for? . . . I give them a lot of leeway. I'll do my best to let them do things on their own. They know the students better than I do. ... I ask their opinion and we try to do things by consensus whenever we can. (BK, 12/11) 
Ms. Gann agreed, saying that Kendall supported teacher efforts to experiment in the classroom. "He gives you the opportunity and if it works, then he'll back you up on that. And he also gives you the encouragement to do it" (SG, 1/8). Ms. Gergan said Kendall invites teacher input in major decisions:

He's not one that just says, 'It's my way or the highway.' He will listen to reason. He listens to what teachers say to him before he makes decisions. It doesn't mean he's always going to go with what they say, but he allows all of us to be heard. We are heard. (CG, 1/15)

According to Ms Turner, Kendall "is not a dictator. He's a supporter" (KT, 1/8).

He involved the teachers in articulating the mission of the school:

We've created a shared vision of where we want our school to go, and he's trying to make sure we have the resources, the materials and to protect that vision when he sees things that maybe hinder it, or when we see roadblocks along the way, he's going to try to protect the vision and figure out what we need to do. But I don't see him as the big brother looking over my shoulder to see what I'm doing wrong. He's looking for what I am doing right, or if I'm not doing right, what does he need to provide for me so that I will get it right. (KT, 1/8)

Turner reported that Kendall had become more tactful in other ways, more carefully tailoring his strategies to individual teachers and inviting more teacher input before making certain decisions.

He's learned to think before he speaks sometimes. Maybe it just comes from learning people. He's gotten much more adept faculty-wise and probably parentwise, knowing what you can say to what person. Because what you can say to me, if you said it to my dear friend, Ms. Dana, it might devastate her and she'd go home for a week in tears. He would take her hurt feelings to heart because that's not what he wants. (KT, 1/8)

Turner described operational decisions Kendall made in his first year as principal that did not go well, such as proposed changes in the student dress code or his request that some teachers switch classrooms. "There was a whole big uproar, and he backed off and said, 'Forget it.' But then the next year we got the e-mail that said, 'Thou shalt move 
your things to this person's room.' [Laughs]. And we all just went, 'Okay, here we go,' and we packed up and moved" (KT, 1/8). Kendall simultaneously became more adept at negotiating potentially controversial issues and making clear directives for school-wide changes.

Kendall agreed with the teachers that he invited more teacher input in decision making than when he began as principal:

You get more bees with honey. I don't know that I use more honey these days, but I think I talk to them more before making decisions. I get their opinions. Not that their opinions didn't matter before, but I think now I trust their opinions. . . . When I started I didn't ask anybody anything. It wasn't that I learned that I better do it, I just decided it worked best for me. In the beginning, I didn't know who to get input from. As I got to know people more, I knew which people I should talk to ... I make better decisions based on my conversations with people and thinking things through instead of making an off-the-cuff decision. I have grown wiser to taking my time. I used to think I had to make my mind up right now. I don't anymore. It's alright to wait. (BK, 12/16)

As his practical knowledge of primary education increased, Kendall also became more confident in his instructional leadership. "[Primary] was totally different from my middle school background," he said. "I think after nine years of it, I feel like I've got a pretty good grasp of that area" (BK, 12/16).

Effects on Teachers. According to Ms. Gann, teachers experienced job satisfaction because Kendall encouraged their autonomy and input, which in turn, contributed to higher student achievement: "The teachers feel good, so the students are going to feel good:"

That's why there's such a good learning environment here.... When you are in an environment that you feel stressed or you constantly feel like you are being watched and you're going to be hammered on, you're going to find it a really tense situation for teachers because they are all going to be complaining. I just don't hear much complaining here. (SG, 1/8) 
To summarize Case Study C, for Research Question 1, data revealed that Bill Kendall used five theories of practice that accounted for his instructional leadership behaviors, including the following: (a) building strong personal relationships with and among teachers, (b) unifying teachers to implement state and district directives, (c) providing feedback, (d) encouraging teacher professional growth, and (e) encouraging teacher autonomy and inviting teacher input.

For Research Question 2, data indicated that teachers responded to Kendall's leadership in the following ways: (a) teachers felt valued and affirmed, identified strongly with the school, and shared a sense of community, (b) teachers embraced the school's mission and attempted to faithfully carry out initiatives and directives, (c) teachers experienced a sense of responsibility for improving their instruction and enjoyed the school's success, and (d) teachers believed they were responsible for student outcomes.

Finally, for Research Question 3, Kendall, like the principals in Case Studies A and $\mathrm{B}$, demonstrated reflection-in-action in that during his years as principal he had learned the importance of giving directive, corrective feedback when his preferred methods of modeling and using praise to encourage teacher effectiveness failed to work.

\section{Case Study D}

Betsy Master: Relentless Improvement and Innovation

Betsy Master, principal of D.A. Malone Middle School, was a 29-year veteran of education. A former elementary school teacher, Master spent 14 years as a guidance counselor, assistant principal and finally principal at the elementary level in a nearby district. She became principal at D.A. Malone Middle 13 years ago and presided over a 
long span of high student achievement. The school's academic index rose from 76.6 in the biennium ending in 2002, to 79.9 in 2004, and 86.7 in 2006 .

Master's primary instructional leadership theory of practice involved a relentless, passionate focus on continuous teacher growth and professional learning. Mary Vintner, who taught at D.A. Malone for 23 years and served on the School Based Decision Making Council (SBDM) that hired Master, said she came to the school with a strong reputation for instructional innovation, and that she had made her mark at the school by maintaining this unwavering focus. "We saw her as an innovator, as someone who would come in with great new ideas. And it just about killed some of us!" (MV, 2/25). Despite the pressures inherent in Master's laser-like attention to on-going staff development, teachers at D.A. Malone expressed admiration for her, and pointed to other theories of practice that characterized her instructional leadership, including welcoming teacher input and taking a hands-on approach to dealing with at-risk students.

\section{School Context}

Part of an independent, city school system of approximately 2,250 students, D.A. Malone Middle School served 530 students in grades six through eight. D.A. Malone (usually referred to simply as "D.A." by staff) was the single middle school for the district, receiving students from two area elementary schools and feeding students into one district high school. Located in a neighborhood of blended residential and commercial property in a city of 24,000, D.A. Malone occupied the former high school building and sat flush with one of the two elementary schools, sharing a parking lot and a continuous hallway that ran through both buildings. 
The independent school district was surrounded by a larger county district of 13,000 students. Like many similar communities, the city and county schools struggled for many years over annexation issues and students who desired to attend schools out of their assigned district. By mutual agreement, during the year of this study the districts committed to allowing 100 students per district to attend the other district's schools, not counting children of full-time employees. The numbers were scheduled to rise at a fixed rate over a period of several years. Ms. Master described a general public perception that the independent schools were "elitist" because of the desire of out-of-district students to attend there, but she argued demographics debunked this myth. While the percentages of minority and English Language Learner (ELL) students was relatively low, 45\% of D.A. Malone students received free or reduced lunch.

Master acknowledged, however, that the climate and culture of the city schools, including D.A. Malone, did foster and encourage an expectation of high achievement on the part of students. She cited the small size of the district as part of the explanation:

We know a lot of the children's families because we've had older siblings. We also have the ability to communicate with the two elementary schools.... I think communication between the administrators at all the buildings, including the high school, is very strong. We share a lot about our children. We do know a lot of the children's families and many of their parents attended the city schools, so there's a real understanding of our system both behaviorally and academically. (BM, 2/19)

Parents at D.A. Malone held high expectations for the school and for student behavior and learning outcomes. Master cited frequent revisions to the school dress code and parent resistance to a recent effort to revise the grading scale as examples. "The grade revision was a very heated, emotional discussion for change for teachers but also 
from parents who said, 'We do well because we have such tight grades.' That just really sent the message home again the expectations that the parents have" (BM, 2/19).

The teachers at D.A. Malone shared the same expectation for high student achievement and internalized Master's emphasis on on-going professional growth and teaching excellence. The faculty included five teachers with National Board certification and several with counseling degrees. While she regularly monitored teachers through the brief classroom visits Master called "drop-by's," she indicated that her staff actually needed little supervision:

They're an excellent group. I feel extremely fortunate to work with them. I feel like if all I did was sit in my office that the school would go well. I know when I'm in the office teaching goes on. It doesn't change just because I'm out in their classrooms or I'm doing drop-by's that day. ... They do what they do, they know my expectations and I don't really have to call them to task. (BM, 2/19)

Teacher participants agreed that the faculty at D.A. Malone possessed high standards of professionalism. Master's theories of practice sometimes placed demands on teachers, and elements of her leadership were perceived by some to be divisive, but teachers reported a strong faculty culture and an abiding commitment to improving student achievement and instructional innovation.

\section{Betsy Master: Research Findings}

Three theories of practice described Ms. Master's instructional leadership. Each theory of practice is described below, including the core assumptions that made up the foundation of the theory of practice, the action strategies that logically emerged from those assumptions (Research Question 1), and the effects of the theory of practice on teachers (Research Question 2). When data suggested that Master engaged in reflective 
practice by adjusting or modifying her theories of practice by revising her assumptions or action strategies, this process is also described (Research Question 3).

Theory of Practice 1: Promoting Continuous Teacher Professional Growth

Ms. Master understood her primary mission as principal to involve facilitating, encouraging, and monitoring continuous teacher growth and improvement.

Overwhelmingly, teacher participants identify this as Master's key theory of practice:

"She is very much involved in the teaching and learning. She has a lot of innovative ideas and has encouraged us in lots of different ways to try new techniques" (MV, 2/22). "I see her as our curriculum leader" (TP, 3/3). "I think of her primary role here as instructional leader.... She's concerned with everyone being the best teacher they can be" (AS, 3/3).

Master promoted teacher learning and professional growth by carefully structuring professional development that focused on learning new instructional strategies, and by monitoring teachers' efforts through documentation, classroom visits, and the evaluation process. Master continually shared books, articles, and ideas with teachers through e-mail, during informal conversations, and during formal observation conferences. While some teachers perceived that the burden of continual innovation and documentation was sometimes onerous, they also reported an appreciation for the way Ms. Master approached these topics, and made a genuine effort at experimentation and implementation of new ideas (see Figure 23).

Assumptions. Ms. Master assumed that teachers, like students, needed to practice new skills to internalize them. Teaching was a profession that required continuous 
ASSUMPTIONS

2

For principals evaluate

must be actively

engaged in the

professional growth

process

\begin{tabular}{|l|}
\hline "The only way you can \\
help students learn at \\
high levels is to make \\
sure teachers have the \\
best instructional \\
techniques"
\end{tabular}

ACTION STRATEGIES

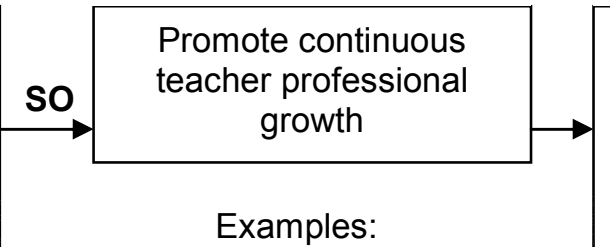

1. Use teacher learning teams to promote and share new teaching ideas

2. Use faculty meeting to explore and review research-based practices

3. Emphasize continuous growth through informal communication and the formal evaluation process
TEACHER EFFECTS

Teachers experiment with new instructional strategies, are open to new ideas, and faithfully carry out instructional initiatives

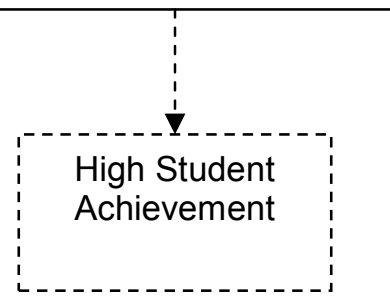

Figure 23. Betsy Master, Theory of Practice 1, for encouraging continual professional growth, including assumptions, action strategies and teacher effects. A tacit assumption of all instructional leadership theories of practice is that these action strategies will indirectly result in higher student achievement, though this link is not explored in this study (indicated by dashed lines in the figure).

learning and growth to be effective, and her role as principal was to encourage that

process of on-going professional development:

That's the only way you can help the students achieve at high levels is to make sure that your faculty understands the best instructional techniques to utilize and has at their fingertips the resources they need to help them do their job best. If you don't have what you really need [in terms of professional knowledge] to do the job to the best of your ability and to motivate the children and to make those connections to their world, then you are sort of lost before you begin. (BM, 2/19)

Master believed that to promote continuous teacher learning, she had to be a

model learner herself, also engaged in professional development and growing her

knowledge of instructional strategies.

I don't understand how you can evaluate a teacher on what they're doing unless you fully understand where they're coming from. I wouldn't expect a teacher to be able to go in and pick up and use something without having that knowledge myself to be able to do it. That's supposed to be my role, to help guide and help them grow. (BM, 2/19) 
Echoing Master's beliefs, Terri Peroni, who taught for 14 years at D.A. Malone, expressed similar assumptions about the importance and necessity of ongoing professional learning:

Self-assessment is very important for all of us. We need to look and say, 'How can I grow?' If you think you've arrived, you may get real static and status quo and not be open to new ideas. It's important for all of us to continue to grow. We [teachers at D.A. Malone] are continuously learning. (TP, 3/3)

Amy Seton, a 20-year teaching veteran with 10 years of experience at D.A.

Malone, pointed to the principal's accountability for promoting higher student

achievement as the source of Master's assumptions. "Everything in our state and many

other states is completely test score driven, and she feels validated by the test scores

being high. That's her only method of impacting test scores. As a principal, what else

can you do? You can't get in there and teach the kids yourself' (AS, 3/3).

Mary Vintner, who served on the SBDM Council that hired Master, said the principal's focus on continuous teacher learning was consistent with the qualities appealing about her as a candidate for the position. "When she was hired it was because of her innovation. She seemed to be innovative, and so I guess she is. Perhaps she's willing to take risks because it [experimenting with new instructional approaches] is kind of risky" (MV, 2/22).

Action Strategies. Ms. Master used a variety of action strategies to promote teacher growth, including teacher learning teams, whole-faculty professional development, informal communication, and emphasizing new approaches through the formal teacher evaluation process. She played a key role in the process by facilitating 
and modeling new instructional strategies and participating in professional development alongside teachers.

D.A. Malone, like schools throughout the Red River Regional Education Cooperative, spent three years participating in an intensive professional development program called Thoughtful Classroom, in which small teams of teachers (called "learning clubs") met to learn, explore, and share their experiences using new instructional strategies based on the classroom effectiveness research of Marzano, Pickering, and Pollock (2001). Master introduced Marzano's work to her staff several years before the Thoughtful Classroom initiative began as part of a two-year, district-wide professional development agenda. "We [district principals and administrators] went out to Phoenix and saw Marzano and five or six others and we came back saying, "Whew, this is where we want to go,' and that started our study of Marzano" (BM, 2/19). Master distributed Marzano's books to all the staff and did activities with the entire faculty:

We all read the book, and then I broke them down into groups, not necessarily by content. Each group took a section of the book and they had to present to the faculty what their section was about. And there were some strategies that we presented as principals. We all [district principals] met together and came up with handouts and activities we wanted our teachers to do, so everybody was sort of hearing the same message, but they might tweak it just a little bit based upon the [needs of] faculty. (BM, 2/19)

Master then monitored the teachers' progress in utilizing Marzano's strategies through the evaluation process and informal classroom walkthroughs (MV, 2/22; BM, 2/19; Observation, 2/25). Master called these brief classroom visits “drop-by's," and usually completed a feedback sheet for the teacher with each visit: "Our drop-by forms include a lot of the language of Marzano, what things did you see going on, what were 
the teachers doing, what were the children doing? These are best practice strategies, check off what you saw" (BM, 2/19).

The two-year Marzano study prepared teachers for Thoughtful Classroom because they were already familiar with the key ideas behind the instructional strategies (BM, 2/19). Long before Thoughtful Classroom, Master also set up a similar structure of learning teams to explore new strategies (TP, 3/3; AS, 3/3). Master designated one teacher from each content area to serve as a lead teacher, and this core group met with her to learn new instructional or assessment techniques, and then shared those techniques with other teachers in their areas. Lead teachers met monthly, and then met with their teams two weeks later to share new strategies, or to debrief teacher experiences using the strategies. This format foreshadowed the teacher "learning clubs" integral to Thoughtful Classroom, and Master maintained the Lead Teacher structure to deliver Thoughtful Classroom PD experiences.

Ms. Peroni, who served as one of the lead teachers, described the process:

We [the lead teachers] would model the strategies first, and then we would bring it to our teams and then the team would take it to their classroom and then try it and bring samples of student work and share how it worked with the students. For example, we just did open response training ... We have to show Ms. Master how students scored. ... Each department has to turn in their open response and she will look at them and give us feedback on that. (TP, 3/3)

While the lead teachers continued to facilitate a portion of the school's professional development, Master also regularly utilized faculty meetings to model or review instructional strategies. "I don't do it at every faculty meeting, but I try to model good instructional strategies” (BM, 2/19). Faculty meetings at D.A. Malone were far more focused on professional development than on conducting routine business, according to Ms. Seton: 
She models things for us in faculty meetings that she's seen in other places. This is really great for middle school - ways to make basic things more fun, the basic stuff you have to do no matter what - how can you do it and be more engaging. Just to switch things up and not be bored. I've really used a lot of those things she's taught us. (AS, 3/3)

Beyond the learning teams and faculty meetings, Master shared new ideas from books and research articles with teachers informally and during formal evaluation processes. "She's very good at making sure we're on the cutting edge of research," Ms. Peroni shared. "She's really good at making sure that we have the latest readings on effective instructional practice. She keeps up with all that and makes copies and puts them in our mailboxes" (TP, 3/3). Ms. Seton agreed: "She shares with us a lot of articles she reads, instructional techniques. When she goes to a workshop, she'll come back and share things with us and reviews" (AS, 3/3).

Master described this ongoing sharing as a routine part of her work, reflecting her level of involvement in professional associations and her own efforts to keep pace with new knowledge regarding effective middle grades practices:

I send them copies of articles that I find in the two middle educators' magazines. There's the National Middle School [Association's publication] and the secondary principal's association [National Association of Secondary School Principals], they have a middle school publication that I get, and an ASCD [Association for Supervision and Curriculum Development] that I get and read. . . I've got all kinds of books that I'll loan out. If I'm reading something online and I think it will be good for one person, I'll e-mail it to them, "Thought you might be interested in this," or, "Here's a good place to get some materials." A lot of times they'll send me back things, "Have you seen this website?" So I do think that's important in maintaining an instructional focus. (BM, 2/19)

The formal evaluation process also served as a vehicle to emphasize continuous teacher learning. All three teacher participants mentioned the importance Master placed on the Professional Growth Plans the district required teachers to initiate and maintain throughout the school year (MV, 2/22; AS, 3/3; TP, 3/3). During an observation for this 
study, Master met with a teacher to do her formal evaluation conference. Master used equal portions of praise and suggestions, offering ideas for materials and techniques to meet the needs of struggling students and ways to more effectively collaborate with other teachers. The meeting included a review and discussion of the teacher's growth plan, and Master suggested ways the teacher could organize time to achieve some of the unmet goals of her growth plan (Observation, 2/25).

Teachers also cited Master's requirement that they produce documentation demonstrating their use of new instructional techniques or their efforts to improve student achievement as a key component of her efforts to monitor their progress (AS, 3/3; MV, 2/22). In the past, teachers submitted examples of student work, evidence of instructional strategies, unit plans, and assessments. During the year of this study, Master emphasized open response items, the student writing tasks that were a key component of the Kentucky Core Content Test (KCCT). She required teachers to share and discuss open response prompts and samples of student work with their learning teams, and submit the results to the principal (BM, 2/19; MV, 2/22; TP, 3/3; AS, 3/3). According to Ms. Seton, besides enhancing student performance on open response, this approach was consistent with Master's long-standing emphasis on making assessment guide instructional planning:

We've been turning in tests [to her prior to this year]. She wants us to write our test before we teach a unit and turn it in to her. Not that she's really looking over it or doing anything with it, it's just the fact that we know where we're going before we go there. She wants to make sure we're doing it in that order, and if we have to turn it in that kind of forces us to do it in that order. So that's a good strategy on her part. (AS, 3/3) 
Ms. Vintner was overwhelmed in the past by the burden of implementing and documenting so many new strategies and assessment techniques, but in recent years the workload had lightened considerably:

She still requires documentation, and I must admit that in the past . . . we have been required to do so much documenting of new techniques and strategies that it was overwhelming. Absolutely too much to do. Forget having a life, you've got to do all this documenting.... She requires a whole lot more than other principals in other districts and maybe even other principals in this district, [but] that has lightened up some. (MV, 2/22)

Vintner speculated that at times when the level of documentation had been particularly intense, Master was responding to pressures from central office administrators to be more aggressive in monitoring teacher activities. Ms. Seton echoed Vintner in saying that the workload had at times been too much for teachers to handle, and confirmed the perception that the central office was to blame:

We have had points at which we've been overwhelmed with too many new things at once. It was a district-wide thing, not just our school, where we felt that with the Thoughtful Ed program, there were so many components to that and we were getting too much at one time and there was so much to keep up with and we're supposed to continue doing the things we learned last year, but here are four or five new things and we want to see examples of them and samples of them. Several of us complained about it straight to the staff at the central office and they listened and backed up. So it was fixed as soon as possible. (AS, 3/3)

Master agreed that changes in style at the central office did make a difference in how teachers experienced the level of monitoring and supervision for their work. "I don't think that we're doing any less than what we did when other superintendents have been here, it's just different. The expectations are still there and they know what our goal is going to be, but maybe there's not as many people looking over their shoulders, and that's what the teachers feel" (BM, 3/19). She shared their frustrations and sometimes 
even the sense of being overwhelmed, and had taken specific steps to focus teacher

professional growth efforts based on teacher feedback:

I'm not sure in education how you avoid that feeling [of being overwhelmed] because everything is coming at you so quickly. You've got to do this for No Child Left Behind, and you've got to do this for the CATS, and here's another curriculum revision ... changes in assessment. ... They all feel that way and part of my role is to help them understand I feel that as well and by being together and working together, we're just going to tackle things as best we can. If they get to a point where they feel like we can't take it any more then we'll stop and take a breath. We've sort of done that with our faculty meetings this year. Instead of doing as much PD we've sort of taken a step back and we've really delved more into, "Let's look at individual student performance ... you all take care of the PD [in your learning teams]." I hope that helps, because we did talk about that at the end of last year with the lead teachers ... and in faculty meetings. (BM, 3/19)

Ms. Peroni said the lead teacher format served as a forum for teachers to express

their concerns about new initiatives, and confirmed that Master responded to feedback

about workload. In this way, the lead teachers served as "buffers:"

[Teachers on my team would say,] "Terri, hold up. We have this and this and this and if you add one more thing that's too much on my plate. Can you help?" ... Lead teachers meet with Ms. Master on a regular basis. We said, "Okay, this is what we're feeling and this is the feedback that's coming from the staff ... it's coming at us too fast. Can we just slow down a little bit and just perfect one or two strategies instead of being exposed to six or seven and not have one or two down really pat?" (TP, 3/3)

Master continued to use faculty meetings to review and model strategies, but left the main work of professional development to the learning teams, which were now focused more on assessment than new instructional techniques. "We've played games and had a lot of fun with it.... I break them into groups sometimes and model [techniques we've previously learned, telling them,] 'By the way, when you all got into groups this is the strategy we used, see if you can use that in your classroom"' (BM, 3/19). 
Master's involvement with professional development and her willingness to participate in learning alongside teachers was a significant part of her instructional leadership, according to teachers. "She attends professional development with us," Ms. Seton reported. "She seems to put in a lot of time before the professional development learning about what's going to be taught. She comes in prepared" (AS, 3/3). According to Ms. Peroni, Master regularly attended learning team meetings, and her "input is great. A lot of times she can come in and see something from a different perspective, which is always good. I love for her to come into our team meetings. Sometimes she gives us fresh ideas that we just didn't think of' (TP, 3/3).

Effects on Teachers. Because of her efforts to promote continuous professional learning, teachers at D.A. Malone reported a high degree of instructional innovation and faithfully tried to implement school and district initiatives. Ms. Master's goal was that teachers "keep an open mind, that they continue to grow and change and that they understand the things I am sharing with them are not . . just another idea. That I wouldn't ask them to grow any more than what I do myself' (BM, 3/19).

Ms. Vintner, who described herself as being from the "Stone Age," said the school "has changed tremendously since Ms. Master came here," and that teachers at D.A. Malone embraced the spirit of innovation, even when it was difficult for veterans like herself to change their practices. "I'm for improving all the time. I'm all the time trying to be better" (MV, 2/22). Vintner gave personal examples of how she had been pleasantly surprised after implementing some of Master's suggestions regarding her classroom management and instructional approaches (MV, 2/22). 
One year, Master asked teachers to complete unit plans using an approach called "layered curriculum," in which students selected from a menu of learning tasks and contracted with the teacher for a grade. While Vintner was skeptical, the results were positive:

Once again, I was surprised because I didn't think that anybody was going to learn anything. I counted that unit as a complete loss. I looked around the room and I saw a lot of movement, a lot of activity. I saw a lot of kids wasting time and it just irked me. I could hardly stand it. Yet, at the end of the unit, I found that there was a lot more learning that took place than I'd ever dreamed. . . . So she's given me some really pleasant surprises along the way. ... Other teachers have continued to do that and do layered curriculum . . . maybe all the time. (MV, 2/22)

Teachers appreciated that while Master had clear expectations that they experiment with new strategies, she gave some leeway in allowing them to continue using only those strategies that matched best with their teaching styles and the needs of the students, according to Vintner: "One of the nice things has been that she has let us feel free that after we've tried something new . . . if we don't like it, if it doesn't fit us, then we feel free not to continue it" (MV, 2/22).

Master confirmed that while she wanted to see every teacher using research-based strategies, specific approaches could be modified to meet individual teacher and student needs:

I've always told them, they're professionals, they're trained. Not every strategy works for every style of teaching. As long as you're trying and you're looking for things that really motivate the children, that reach them in the ways you want to reach them, then that's great. That's what you should be doing. But certainly I don't want anyone to adopt a procedure just because I said that was the way it needed to be done. (BM, 3/19)

Teachers reported that the faculty at D.A. Malone were highly professional and required little direction to carry out the new ideas Master introduced. Teachers "really do 
attempt to do the new things she introduces and most will follow through and continue to do them long after," according to Ms. Seton. "The secret to our school's success is that kind of commitment from the teachers. Her leadership has evolved around that kind of climate here" (AS, 3/3). Ms. Peroni agreed:

Teachers here really care about their work. Betsy is a role model. She has high standards and high expectations. If you have high standards, people will try to measure up to those standards, just like [the high standards] we have for our students. . . . We want to make sure we raise everybody up. ... That's evident throughout the building. If you go to any room in the whole building, you will see they [teachers] have confidence and faith in Betsy to be our instructional leader, to be able to know that we're doing what we've been hired to do. We're doing best practices. We're doing what we need to do to help students grow and be productive citizens. (TP, 3/3)

To summarize, Betsy Master's first theory of practice emerged from assumptions that for students to achieve at high levels teachers must continually hone and improve their practice. She understood her primary role as principal to involve encouraging and facilitating this process of continuous teacher growth. Based on these assumptions, Master used action strategies including establishing teacher learning teams to promote and share new instructional ideas, using faculty meetings to explore and review research-based practices, and emphasizing on-going improvement through informal communication and the formal evaluation process. The key effect of her theory of practice was that teachers experimented with new instructional approaches and attempted to faithfully carry out new initiatives.

\section{Theory of Practice 2: Inviting Teacher Input}

While she never rejected the importance of inviting teacher input into decision

making, over the years Ms. Master developed a more intentional focus on communicating 
with teachers and actively soliciting their ideas and opinions. Teachers identified this as one of the key changes in her leadership during her 14-year tenure as principal. Master invited teacher input by informally asking for feedback on various ideas and initiatives, and by facilitating discussions and debates among teachers in school-wide meetings. Teacher participants believed Master sincerely desired teacher input, but described the perception on the part of some staff that there was an in-group of teachers who had influence with the principal, and an out-group of teachers whose voices were not really heard, while the vast majority of teachers fell somewhere in the middle. Master viewed this as a by-product of a particularly difficult dispute with a teacher some years before who subsequently left. She learned from the episode the critical importance of actively engaging teachers in decision-making. The incident remained vivid in the memories of teachers as well, who expressed regret that some teachers might not fully voice their opinions for fear of being placed with the out-group. They genuinely believed that Master desired input, and believed that most teachers did and should openly express their desires and opinions (see Figure 24).

Assumptions. Ms. Master described how she had learned the importance of carefully communicating with teachers. While she never dismissed or rejected teacher input, she had learned over the years that it was vitally important to keep teachers in close communication and solicit their ideas and opinions. As a result, she believed that teachers were more engaged in decision-making and more satisfied with their work environment. "They're just happier," she explained, and believed that happier teachers would be more effective in serving students and promoting higher levels of achievement (BK, 2/19). 
ASSUMPTIONS

2

When teachers don't feel

that lines of

communication are open, negative climate issues can result

Teachers are happier and more effective when they participate in decision-making and their opinions are valued
ACTION STRATEGIES

TEACHER EFFECTS

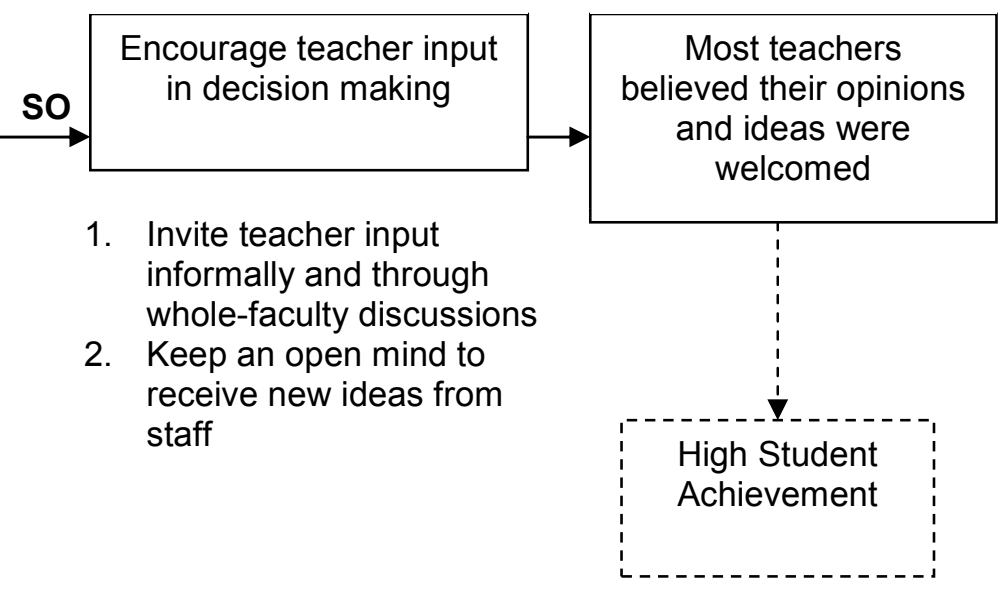

Figure 24. Betsy Master, Theory of Practice 2, for inviting teacher input, including assumptions, action strategies and teacher effects. A tacit assumption of all instructional leadership theories of practice is that these action strategies will indirectly result in higher student achievement, though this link is not explored in this study (indicated by dashed lines in the figure).

She confirmed teacher reports that her attention to communication and collaborative decision-making had grown over the years. In the beginning of her principalship, it would not have emerged as one of her key theories of practice (BK, 3/19). "I always thought I was pretty open-minded, but then I had something come up and bite me on the face and didn't quite go the way I thought it would go, so I've learned that maybe I wasn't getting as much input as I thought I was" (BK, 2/19).

Master described her decision several years ago to require all language arts teachers to teach a seventh grade writing portfolio class, believing that by engaging more teachers in this key element of middle school accountability, student scores would increase. Master informed an individual teacher before she announced this change to the 
faculty as a whole, and the teacher quickly reported the news to others. As Master remembered the story, a loud outcry ensued, led by one talented and successful but particularly strong-willed teacher. The faculty divided, and teachers accused her of making decisions without inviting input and practicing poor communication. The most resistant teacher continued to foment dissension, and the situation came to a head when the superintendent addressed the entire faculty about their problems with communication, teamwork, and support. The next year, the teacher left D.A. Malone and took a position in another district (BK, 2/19; 3/19).

Master had no regrets about the decision to reassign teachers or about the loss of the dissenting teacher, but learned from the feedback of others that she should be more careful about communicating with faculty and engaging them in major decisions:

I made that decision on my own ... and it all kind of just blew up. In retrospect, that was one of the things that helped me change and grow because I did go to the superintendent - well, I was actually brought to the superintendent, and we did talk about it and I did get some input from other administrators who saw things differently and could see more objectively than I did. I made some changes in how I did things. So although at the time, going through that, it wasn't the most pleasant thing, but it was an opportunity for me to grow and learn. (BK, 2/19)

Master credited the advice of her superintendent as a source of new understanding for how to improve her effectiveness. "What I was asked to look at was, "Are you communicating at the level you think you are? Maybe we need to be looking at some different ways to do that and take some of the things that were causing a little bit of turmoil and be more proactive"” (BK, 2/19). As a result, Master made intentional efforts to communicate with staff and involve teachers in key decisions.

Action Strategies. Ms. Master used both informal means of communicating with teachers and inviting input, as well as facilitating whole-faculty discussions during 
meetings. E-mail was one of the informal means she used to solicit teacher opinions, but Master also valued the practice of talking to "key people" to share their insights, asking them, "What are you hearing?"

I don't ask them to divulge their sources, but they understand and I've told some of them, "I can't be out there, I can't be in the lounge with every lunch group, I can't be with you when you're talking in your department meetings. So I'm going to rely on you. If things aren't going well, you need to come and let me know because unless I get a sense of it, how do I know to make a change or to bring something up?" Or I'll send a generic e-mail, "Just wondering about this." Because sometimes that's all it takes is just to open that up, "I know you are all talking about this," so I'll just sort of throw it out there. "Has anybody thought about this? Please let me know." I think that's critical. (BK, 2/19)

If faculty meetings were not dedicated to professional development, Master often used them as a forum to discuss topics such as behavior expectations, school-wide intervention and support programs, and scheduling. "Sometimes I'll just ask them to write down their thoughts. 'Last year I had a lot of complaints about when your planning period is, so I want everybody to write down one or two times you'd really like to have planning. I'll try, but I can't promise'” (BK, 2/19).

Faculty meetings were productive in this sense because Master genuinely seemed to be open to teacher suggestions and input according to Ms. Vintner. "I think she does a marvelous job with her faculty meetings. She does not come in with it [a decision] already set. She's very patient [with faculty discussion]. We talk some things to death!" (MV, 2/25).

Ms. Peroni confirmed that this had been a growth area for Master, but echoed Vintner is stating that the principal was now genuinely open to the input of staff: "I've seen her communication improve. When she's implementing a policy, she makes sure we are stakeholders. She solicits our ideas" (TP, 3/3). 
Effects on Teachers. Ms. Master hoped her invitation of teacher input fostered a staff culture that was open to discussion, dialogue, and sharing new ideas:

It's being able to hire people who have similar values that you have and a similar philosophy, but not exactly the same because then you don't get debates in faculty meetings about whether this is a good idea or that's a good idea ... the impact you have in the openness of teachers to different ways of looking at things. It's not my way or the highway, it's, "Here are a variety of ideas, but know that the key expectation is that we keep growing." (BM, 2/19)

Teachers enjoyed the autonomy that accompanied Master's openness to input:

"One thing I appreciate about her is that if she has an idea and she tosses it out and nobody grabs it and runs with it, generally speaking, she's not mad or upset," Ms. Vintner explained. "She'll just get another idea and toss that one out. I think that's really good" (MV, 2/25). Ms. Peroni agreed: "Ms. Master really wants to hear our opinions about situations and she makes everybody feel very comfortable with different ideas, even if they're different from her ideas or her perspective" (TP, 3/3).

Past divisions within the faculty continued to shape some teacher perceptions about Master's welcoming of input. Among the three teacher participants interviewed for this study, two (Seton and Vintner) both described how some teachers perceived an "ingroup" (the phrase was the researcher's suggestion, not the words used by participants) of staff who had a strong influence on Ms. Master, and an "out-group" of faculty who had little influence with the principal and whose opinions were not viewed as meaningful. One (Peroni) claimed to have no knowledge of this perception.

Seton elaborated: "I would add another group — an in-group, an out-group, and an 'I'll listen to you' group, individuals who might not necessarily be in or out but still have impact if they will speak up" (AS, 3/3). Seton speculated teachers in the out-group were 
not adept at voicing a dissenting viewpoint without sounding disagreeable and negative, and that Master "tuned them out" as a result:

However, their opposing opinions might really have some validity, they're just not good at expressing it. Instead of being trained in how to express it better, or guided in how to express it ... it's perceived as criticism. That's because almost anybody would perceive it as criticism. These particular teachers . . . don't do a good job of it. (AS, 3/3)

Seton recalled the same event Master described in which a well-regarded teacher vocally opposed the principal's plan to assign all language arts teachers to a seventh grade writing portfolio class. Seton perceived it as unwillingness on Master's part to compromise. "No compromise was allowed. That's rare. That's not typical of Ms. Master. ... But in that particular case she just chose not to. I hate that we lost that teacher, but life goes on. I think that negatively impacted the climate for awhile" (AS, 3/3). Seton believed the incident had a dampening effect on faculty input because some teachers later feared that if they spoke up, they would be regarded like the outspoken teacher who eventually left.

Master denied there was an "in-group" of teachers who had greater influence on her, but did acknowledge some teachers might view themselves as "out-group" because of their ineffective communication styles. She believed this was a misperception, however, and insisted that the level of staff dialogue revealed few teachers who were afraid to express their opinions:

It's interesting because the last few faculty meetings we've had have been pretty open. Nobody was quiet about anything we were talking about [at our last meeting]. . . Other staff come to me privately instead of voicing their concerns in an open meeting. . . . When I've gone around and asked individually [for teacher opinions], I've never had anyone refuse to give me their input. (BM, 3/19) 
Master expressed no regrets for how she handled the class assignment situation. The teacher who subsequently left had a toxic impact on staff culture and morale, and in retrospect thought that many problems in faculty communication were related to her polarizing effect on other teachers. "At the time I wouldn't have thought that, but after the teacher left, it was almost like other teachers said, 'Okay, I can now say something and I'm not going to be cornered for showing support [of the principal]."' Master did believe, however, that she and the faculty grew considerably from that experience and now placed a greater value on the role of teacher input and communication. "Going through all that together and deciding we need to pull together because if we don't we're all going to divide and die on the vine, I think that makes a difference too in how they perceive [teacher input today]" (BM, 3/19).

Master's second theory of practice emerged from her assumption that teachers are happier and more effective when they participate in decision making and believe their opinions are valued. When teachers don't perceive they have a voice, negative climate issues result. Master learned this the hard way, from critical teacher feedback that suggested she was not making a priority of inviting teacher input. This did not necessarily constitute double-loop learning, in that Master asserted that she always assumed input was important, but her change of emphasis did indicate reflection-inaction. She was not achieving the learning climate she desired, and so she gave more energy to soliciting teacher feedback informally and in whole-faculty formats. Teachers at D.A. Malone perceived lingering effects of past failures in communication, but believed Master sincerely wanted their input. They were empowered to offer their ideas and opinions and participate in decision making. 
Theory of Practice 3: Engaging with Students

Ms. Master assumed that for students to be successful, educators must take responsibility for initiating and maintaining personal relationships with them. Master intentionally engaged students on a variety of levels, and took an active role in identifying students who needed extra help, and personally communicated with students and parents. She facilitated intervention activities for students, and teachers appreciated her efforts because these activities lightened the workload for them and had a direct impact on student achievement (see Figure 25).

ASSUMPTIONS

The principal plays a key
role in engaging with
students and facilitating
discussion about
individual student needs

1

Educators must be actively involved with students on a personal level in order to identify and carry out effective interventions to promote higher student achievement

\section{ACTION STRATEGIES}

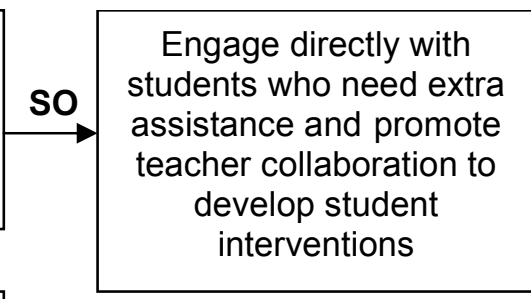

1. Communicate directly with parents and students about individual student needs

2. Work one-on-one with students who need extra assistance

3. Facilitate grade-level teacher teams to identify at-risk students and develop interventions

\section{TEACHER EFFECTS}

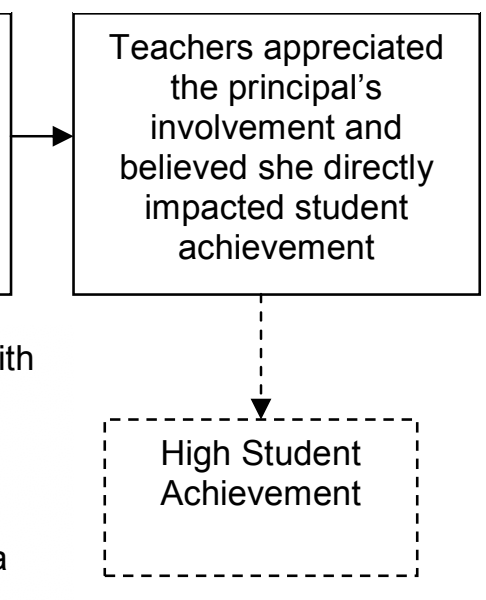

the principal's involvement and elieved she directly mpacted student achievement 
learning. "My job is to see that children work to as much of their potential as possible" (BM, 2/19):

They're here and my responsibility is to see while they're here that they have a safe place to attend school, a staff that's very competent in what they're doing, very knowledgeable in their content and how to instruct that content and the expectation that they have to get the best education they can, and then by golly I'm going to make sure they [the students] do their part too.... Sometimes you talk to a child and sometimes that's all it takes is just one conversation ... it's almost a parenting kind of role. (BM, 2/19)

Because different staff members bring different qualities to their work that children respond to differently, Master believed every educator could connect with some students. "Some of them respond very well to my counselor; some don't. . . I can't talk to some of them, but I can with others" (BM, 2/19).

Master viewed engaging students in a way that responded to teacher concerns as a central function of her position and believed she was able to improve student performance as a result. "I just think that's a key part of our role as educators. Letting the teachers come down and say, 'I'm having trouble with so and so, would you mind talking to them?' I just don't see how you can operate without being involved" (BM, 3/19).

Action Strategies. Ms. Master engaged students by directly communicating with them and with their parents about teacher concerns and through facilitating teacher discussion and sharing to develop effective student interventions. "I take a very hands on approach to what the children are doing" (BM, 2/19).

Master frequently reviewed student grades and sent personalized letters to parents of children who were struggling. "I spent the weekend writing letters [to parents]," she said. "Some got generic, 'your grades have dropped, you may be retained' [messages], 
and there were others that needed more than that. They needed something that really spoke to them and their child" (BM, 3/19). D.A. Malone operated a "Friday school" program from 3:00 to 5:00 after school on Fridays for students who needed extra help. Master called in each of the students to notify them of their Friday school assignment and contacted their parents. Ms. Seton said Master's role in the program was a boon to teachers. "When we first started it, the individual teachers who needed them to stay would have to contact the parents to let them know but now she's taken that over and we only have to contact the parent if it's somebody new. If it's somebody on the standing list, she does that for us, which is very nice" (AS, 3/3).

Master also facilitated regular meetings of grade level teachers for the specific purpose of discussing students who needed extra interventions (BM, 2/19; AS, 3/3; TP, 3/3). "I serve as the contact person for the parents, 'This is what we're going to do, here's the plan we've come up with for your child'" (BM, 2/19). Because D.A. Malone did not use the typical team structure of most middle schools, Seton found this practice to be especially helpful for encouraging teacher discussion about student needs. "As a group, we'll discuss what we can do to help them. Do they need Extended School Services? Do they need tutoring? Do they need something as simple as a parent conference?" (AS, 3/3). Master agreed the teacher sharing was an important contribution to the process:

We really try to work on it [engaging in dialogue]. And it's the teachers. It's not the three of us [the administrative team] sitting up here. It's most interesting when they get together and say, "I'm not seeing that in my class." "What are you doing differently?" "Well, here's what I do." That level of discussion helps a lot. (BM, 3/19) 
During observations for this study, Master answered phones in the main office and filled in for an absent attendance clerk, managing student and parent needs and phone calls (Observations, 2/19, 2/25, 3/19). When the guidance counselor was not available, she met with an incoming transfer student to administer a reading placement test and develop the child's schedule. Visiting a language arts class, she assisted individual students in working on writing portfolio pieces. She met with a group of students who were concerned their friend may be bulimic, and chatted with students in the cafeteria (Observation, 2/25). On one occasion, a large board occupied one wall of her office where she was working on the schedule, a task she believed connected her directly with students (Observation, 3/19). "These are key questions for me: Who needs to be in Literacy Plus? Who needs to be in low math? Looking at test scores and those types of things" (BM, 2/19).

Effects on Teachers. Teachers expressed appreciation for the hands-on approach Ms. Master took to engaging individual student needs. Her actions made their jobs more manageable, and had a direct influence on students (AS, 3/3; TP, 3/3; MV , 2/22). Her efforts "take some work load off," Ms. Seton explained. "If students are not doing well, it's not because they were not given the opportunity by students or her. I think that is a key thing she does that really impacts student achievement directly" (AS, 3/3).

Theory of Practice 3 emerged from Master's assumptions that educators must be actively involved with students on a personal level to identify and carry out effective interventions that would promote higher levels of student achievement. As principal, she played a key role in facilitating discussion among teachers to identify student needs. Master used action strategies including communicating directly with at-risk students and 
their parents, working one-on-one with students who needed extra assistance, and facilitating grade-level teacher team meetings to identify students and develop interventions. As a result, teachers found their jobs more manageable and believed Master was having a direct positive effect on student achievement.

To summarize Case Study D, for Research Question 1, data revealed that Betsy Master used three theories of practice that accounted for her instructional leadership behaviors, including the following: (a) promoting continuous teacher learning and growth, (b) inviting teacher input, and (d) engaging with individual students.

For Research Question 2, data indicated that teachers responded to Master's leadership in the following ways: (a) teachers actively embraced continuous professional learning and faithfully implemented new instructional strategies and initiatives, (b) teachers believed Master sincerely desired their input and shared in decision making processes, and (c) teachers admired Master's efforts to identify individual students with special needs, and believed she had a direct impact on student achievement as a result.

Finally, for Research Question 3, Master demonstrated no examples of doubleloop learning. Neither she nor teacher informants from her school could identify occasions when Master questioned or revised her underlying assumptions. Like Rippy and Kendall, however, she did engage in reflective practice by using negative feedback to reflect on her action strategies, and chose to place greater emphasis on inviting teacher feedback to promote a more positive work climate. 


\section{CHAPTER V}

\section{DISCUSSION, IMPLICATIONS, AND CONCLUSIONS}

This chapter includes three major sections: (a) a cross-case analysis and summary of findings from all four case studies, including the theories of practice (Research Question 1), effects on teachers (Research Question 2), and evidence of double-loop learning or reflection in action (Research Question 3); (b) linkages from this study's findings to previous literature; (c) an assessment of the study's results; and (d) suggestions for education stakeholders.

\section{Summary of Findings and Cross-Case Analysis}

The researcher discusses the following in the section below: (a) demographic similarities and differences among the case study principals, and (b) commonalities among the principals' theories of practice, effects on teachers, and evidence of doubleloop learning or reflection-in-action. Tables both summarize the case findings and serve as a vehicle for cross-case analysis.

\section{Demographic Data}

Participant principals were identified by educational leaders from their regional educational cooperative as having a reputation for being self-reflective and possessing a proven record of raising student achievement. The researcher verified that the principals had presided over at least two biennia of steady student gains as measured by Kentucky's Commonwealth Accountability Testing System (CATS). The principals were veteran educators. The least experienced among them had 17 years of experience in education. 
Three of the four principals were in their first and only principalship, but all had served in that role for several years. Three of the four were female; one was male. Their schools represented rural and medium-size city populations, and fell into three different grade configurations (K-6, K-8, and 6-8). Table 1 presents a comparison of descriptive data from the four case study principals and their schools.

Table 1

Demographic Comparison of Case Study Principals

\begin{tabular}{lcccc}
\hline Case Study & A & B & C & D \\
\hline Principal & Edmonds & Rippy & Kendall & Master \\
School & Cane Ridge & Cherrywood & Homestead & D.A. Malone \\
Years as principal & 12 & 7 & 9 & 17 \\
Total years of experience & 30 & 31 & 16 & 29 \\
Grades & K-6 & K-6 & K-8 & $6-8$ \\
Student Population & 430 & 746 & 427 & 550 \\
District Population & 12,000 & 12,000 & 3,700 & 2,300 \\
Biennia Academic Index 2002 & 77.7 & 77.7 & 69.4 & 76.6 \\
Biennia Academic Index 2004 & 91.1 & 90.4 & 84.8 & 79.9 \\
Biennia Academic Index 2006 & 93.2 & 96.8 & 93.4 & 86.7 \\
\hline
\end{tabular}

Commonalities in Theories of Practice (Research Question 1)

Research Question 1 sought to document the instructional leadership theories of practice for the four case study principals, including their underlying assumptions and 
action strategies for improving student achievement. Clear commonalities emerged among principal theories of practice. There were far more similarities in their instructional leadership assumptions and action strategies than differences. Of the eight commonalities described below, all either appeared as a theory of practice or were embedded as an assumption or action strategy within a theory of practice (see Table 2).

\section{Inviting Teacher Input}

For all four principals, inviting teacher input was a key dimension of instructional leadership, making it the most widely used theory of practice. Based on assumptions that the challenges of increasing student achievement were too complex for the principal alone to make all instructional decisions, the participants actively solicited teacher feedback and invited teachers to participate in school governance. Donna Rippy provided an example when she said of herself, "I look at myself as being the leader of the building, but not all-knowing" (DR, 1/22). Principals assumed that because the teachers often had more direct experience working with students and with specific problems, the principals needed the collective wisdom of the faculty to be effective. As one teacher described Marie Edmonds, "She realizes that she cannot know all, experience all, read all about a particular subject or topic. She has some wonderful resources in her building and she is not afraid to use them" (DH, 3/8).

The principals also understood that inviting teacher input played a utilitarian role in promoting higher levels of teacher motivation and job satisfaction. Teachers are "just happier" when they have a role in decision making, according to Betsy Master (BM, 2/19). Donna Rippy agreed: "I believe that people have more buy-in if they're allowed to have input. I believe when people see that you value what they think or how they feel 
Table 2

Commonalities in Principal Theories of Practice

\begin{tabular}{lcccc}
\hline Commonality & Edmonds & Rippy & Kendall & Master \\
\hline $\begin{array}{l}\text { Inviting teacher input } \\
\text { Nurturing personal } \\
\text { relationships }\end{array}$ & $\mathrm{T}$ & $\mathrm{T}$ & $\mathrm{T}$ & $\mathrm{T}$ \\
$\begin{array}{l}\text { Continuous professional } \\
\text { learning }\end{array}$ & $\mathrm{T}$ & $\mathrm{T}$ & $\mathrm{T}$ & $\mathrm{A}$ \\
$\begin{array}{l}\text { Feedback (constructive \& } \\
\text { corrective) }\end{array}$ & $\mathrm{T}$ & $\mathrm{A}$ & $\mathrm{T}$ & $\mathrm{T}$ \\
$\begin{array}{l}\text { Teacher Autonomy } \\
\text { Unifying staff around common }\end{array}$ & $\mathrm{T}$ & $\mathrm{T}$ & $\mathrm{T}$ & $\mathrm{A}$ \\
$\begin{array}{l}\text { mission } \\
\text { Individual student needs }\end{array}$ & $\mathrm{A}$ & $\mathrm{T}$ & $\mathrm{T}$ & $\mathrm{A}$ \\
$\begin{array}{l}\text { Teacher collaboration } \\
\text { Note. T Commonalty energeed }\end{array}$ & $\mathrm{T}$ & $\mathrm{T}$ & $\mathrm{T}$ & $\mathrm{A}$ \\
\hline
\end{tabular}

Note. $\mathrm{T}=$ Commonality emerged as a whole theory of practice; $\mathrm{A}=$ Commonality was embedded as either an assumption or action strategy of a larger theory of practice.

and you take those things into consideration, it's a leg of that positive climate" (DR, 3/12).

While the principals sometimes mentioned the role of the School-Based DecisionMaking (SBDM) Councils, their use of teacher input transcended formal school governance structures like SBDM. Engaging teachers in decision-making suffused the principals' approach to all aspects of leading the school. Principals (a) established ad hoc 
and standing faculty committees to address specific problems, (b) used faculty meeting time to discuss emerging issues, (c) informally engaged teachers in conversation to solicit their feedback, (d) administered surveys to gather teacher ideas, and (e) encouraged teachers to take on leadership roles within the schools. As one teacher reported of Marie Edmonds, "she wants everybody to be a part of this democracy" (JJ, 10/16).

Sometimes teachers were so accustomed to providing input that delicate school climate issues emerged. "I wonder sometimes, can I not make a single decision myself?" Edmonds lamented (ME, 9/25). Participant principals or teachers at all four schools described incidents in the past where groups of teachers were reluctant to accept a principal's decision or expected to have greater input on a specific issue. Three of the four principals indicated that inviting teacher input was probably not one of their key instructional leadership theories of practice in their early years as principal, suggesting that the tension between teacher feedback and principal decision making can serve as a creative dynamic for professional growth and self-reflection. These phenomena are described in greater detail below in the section exploring double-loop learning and reflection-in-action.

Nurturing Positive Personal Relationships

Edmonds, Rippy, and Kendall all used theories of practice built around assumptions and action strategies regarding the importance of personal relationships. While this did not emerge as a theory of practice for Master, she too expressed an assumption in the important role of personal relationships between teachers and students. The principals did not hold these theories of practice as management strategies designed merely to achieve high levels of teacher or student performance. They assumed rather 
that all people possessed inherent dignity and worth and were deserving of respect, fair treatment and consideration. They nurtured positive relationships as a part of their moral code. Darla Hammond, the curriculum coordinator at Cane Ridge Elementary, described Marie Edmonds's theory of practice for nurturing positive relationships:

It is just a part of who she is as a Christian woman. She values people on a personal level. It wouldn't matter if she was a principal, office employee, or stayat-home mom, she cares about others. This is one reason it works for her. There is no planning or forethought in her actions; it comes naturally. (DH, 3/9)

The principals did realize, however, that nurturing positive relationships with teachers could lead to higher levels of teacher performance. "If I think my boss cares about me and the circumstances I'm in, I'm going to do my best to please him," Kendall explained (BK, 12/11). Likewise, building positive relationships among staff and especially with students contributed to positive school-wide effects, including higher levels of student achievement. Teacher Heidi Bowen credited Donna Rippy’s emphasis on warm personal relationships for Cherrywood Elementary's strong test scores: "You can walk down the hall and everybody's as friendly and open and optimistic with every student and I think it's just made a big difference" (HB, 1/25).

Based on these assumptions about the important role of relationships, the principals used action strategies including (a) encouraging faculty socializing, (b) being available and supportive of teachers in times of personal need, (c) offering praise and appreciation for teacher effort and accomplishments, and (d) engaging with students on an individual level.

Encouraging Continuous Professional Learning

Edmonds, Kendall, and Master all used theories of practice that focused on promoting continuous professional learning and growth for teachers and principals. This 
was not an overall theory of practice for Rippy, but she too expressed assumptions about the critical importance of on-going learning within her theory of practice for inviting teacher input and promoting collaboration. Closely related to their assumptions about the complexities of raising student achievement, the principals assumed that because students present ever-more-difficult challenges to the process of schooling, educators must continue to develop new skills and hone and refine their professional knowledge. According to Edmonds, "None of us is smart enough and the challenges are too big to just close the door and figure it out by ourselves" (ME, 9/25). Kendall agreed: "Students change, programs change, ideas change, and we must change to fit the need," (BK, 3/24). Master explained this as a necessity of improving student learning:

The only way you can help the students achieve at high levels is to make sure that your faculty understands the best instructional techniques to utilize and has at their fingertips the resources they need to help them do their job best. If you don't have what you really need [in terms of professional knowledge] to do the job to the best of your ability and to motivate the children and to make those connections to their world, then you are sort of lost before you begin. (BM, 2/19)

Based on these assumptions, the principals promoted continuous professional growth through (a) establishing teacher learning teams for exploring and sharing new instructional strategies, (b) distributing and studying research articles and books with teachers on best practices, (c) informally communicating ideas for professional improvement, (d) requiring documentation of teacher innovation, and (e) using the formal evaluation process to foster teacher self-reflection on their professional practice. All of the principals took an active role in professional development themselves as participants alongside teachers and through attending their own professional conferences and workshops and then sharing new ideas with staff upon their return. Master explained the importance of learning alongside the teachers: 
I don't understand how you can evaluate a teacher on what they're doing unless you fully understand where they're coming from. I wouldn't expect a teacher to be able to go in and pick up and use something without having that knowledge myself to be able to do it. That's supposed to be my role, to help guide and help them grow. (BM, 2/19)

\section{Providing Feedback}

Providing feedback also emerged as a key instructional leadership theory of practice for Edmonds, Rippy, and Kendall. Master used feedback as an action strategy within her larger theory of practice for promoting continuous professional growth. Because of their strong orientation toward personal relationships, Edmonds, Rippy, and Kendall preferred to use positive forms of feedback including modeling, making suggestions, and offering praise. Teachers described how the principals wrote notes of encouragement to them and their spouses, thanking them for their hard work and performance. Modeling was a common action strategy for feedback. Edmonds described her overall theory of practice: "My natural approach is to accentuate the positive, encourage them, invite them, facilitate them visiting someone else's class who is doing it the way I like to see it done" (ME, 12/4).

As a last resort, the principals used corrective forms of feedback, including giving specific directives, pointing out areas of improvement, putting teachers on corrective action plans, and non-renewing untenured teachers. The principals understood this as an unpleasant but necessary part of their obligation to promote continuous teacher improvement. Bill Kendall described it this way:

It's my responsibility to make sure that good instruction is going on in the building, that if I've got someone who is the weak link, it's my responsibility to strengthen them, provide them with the PD [professional development] they need to become a better teacher, or if need be, the person who invites them to leave. (BK, 12/11) 
Because of their strong desire to affirm teachers and their work, using corrective feedback was difficult for these principals. They described the emotional toll in vivid terms, evoking "sleepless nights" and anxiety about the personal cost to the teachers who ended up losing their jobs or faced with the embarrassment of a corrective action plan. Three of the four principals described how in the early years of their principalship, they were reluctant to use such directive forms of feedback, but had grown more confident in correcting teachers because their first responsibility was to the students. "I had to think about my students," Rippy said, recalling her decision to non-renew an untenured teacher. "My babies [the students] weren't going to go through that again" (DR, 1/22). Ultimately, corrective feedback seemed to constitute "special case" theories of practice which the principals only used when their preferred methods of offering praise, encouragement, suggestions, and modeling failed to achieve the desired effect of continuous teacher improvement.

To sum up the commonalities discussed so far, all the case study principals used a theory of practice for inviting teacher input, and most used theories of practice for building positive personal relationships, promoting continuous teacher improvement, and providing feedback to teachers. Other commonalities among their theories of practice are discussed in the next section.

\section{Other Commonalities}

Besides the commonalities discussed so far, other similarities emerged in the case study principals' instructional leadership. While these similarities were not as prominent among the principals' overall theories of practice, certain commonalities existed as 
embedded assumptions or action strategies within other theories of practice for all four principals.

Meeting individual student needs. The study revealed that Edmonds and Master both possessed specific theories of practice that focused on educators' responsibility for meeting the needs of individual students. For Edmonds, this theory of practice strongly shaped her instructional leadership behaviors. She consistently made efforts to communicate and model a vision of learning that celebrated and responded to individual student needs. Master considered engaging with individual students a key responsibility in her role as principal. Rippy and Kendall also shared this assumption, which was embedded in other theories of practice.

Encouraging teacher autonomy. Encouraging teacher autonomy emerged as a theory of practice for Rippy and Kendall, and were embedded action strategies for Edmonds and Master. Based on the same assumptions that prompted the principals to invite teacher input, the principals viewed teachers as professionals and experts who required little directive leadership. Consequently they gave teachers broad freedoms in classroom experimentation and expressed confidence in teacher abilities. "I give teachers a lot of leeway," Kendall explained. "They know the students better than I do" (BK, 12/11).

Unifying staff around a common mission. All four principals understood their role to involve orchestrating teacher activities in a way that maximized the common purpose and mission of the school. Bill Kendall possessed a strong personal sense of responsibility for faithfully meeting the objectives and carrying out the initiatives established for his school by district and state mandates. Donna Rippy's attention was 
fixed on maintaining teacher focus on a common curriculum and preparing elementary students for secondary education. To a lesser extent, Marie Edmonds and Betsy Master also attempted to unify their teachers toward a common goal: celebrating and responding to individual student needs for Edmonds, and continual innovation and professional growth for Master.

Encouraging teacher collaboration. The principals shared assumptions about the importance of teacher collaboration. In all cases, the principals viewed collaboration as the best way to promote continuous teacher growth. According to Edmonds, "Teachers teach best when they are part of a professional learning community and are always learning and supporting each other" (ME, 9/25). As a result, the principals established teacher learning teams, partnered experienced and novice teachers, and encouraged teamwork and mutual teacher support.

To summarize the study's overall findings for Research Question 1, data revealed striking commonalities in principal theories of practice. The following emerged as either entire theories of practice or were embedded as assumptions or action strategies of larger theories of practice: (a) inviting teacher input, (b) building positive personal relationships, (c) providing feedback, (d) promoting continuous teacher professional growth, (e) meeting individual student needs, (f) encouraging teacher autonomy, (g) unifying staff around a common mission, and (h) encouraging teacher collaboration. The next section explores results of Research Question 2, dealing with the effects of the principal theories of practice on teachers. 


\section{Effects of Principal Theories of Practice on Teachers (Research Question 2)}

Research Question 2 explored the effects of principal theories of practice on teachers. Eight effects emerged. Many of these effects were common to three or more of the principals, and sometimes corresponded with multiple theories of action (see Table $3)$.

Table 3

Effects on Teachers

Reported Effects

Edmonds Rippy Kendall Master

Personal sense of responsibility for student learning

Feel valued and affirmed

X $\quad X \quad X$

Affiliation with school

X $\quad X \quad X$

Believe opinions are valued

$\mathrm{X}$

$\mathrm{X}$

$\mathrm{X}$

Desire to meet principal's expectations

X $\quad \mathrm{X} \quad \mathrm{X}$

High level of job satisfaction/motivation

X $\quad X \quad X$

Actively engage in collaboration

X $\quad \mathrm{X}$

Embrace new ideas/keep learning

$\mathrm{X}$

$\mathrm{X}$

Note. $\mathrm{X}=$ Effect reported for school.

Personal Sense of Responsibility for Student Learning

The most consistent effect reported by teachers in all four schools was a personal sense of responsibility for student learning outcomes. Teachers at Cane Ridge Elementary responded to Marie Edmonds's use of modeling and consistent communication of her vision that all students could succeed if educators took full 
responsibility for outcomes. "We can't make excuses," reported Cane Ridge teacher Darla Hammond (DH, 10/16). Likewise at Cherrywood Elementary, "Teachers know there is accountability," according to Helen Bowen, because of Donna Rippy's efforts to maintain teacher focus on the state curriculum and prepare students for secondary education (HB, 1/25). At Homestead Elementary, Carrie Gergan echoed this sentiment in the way she described teacher responses to Bill Kendall's leadership: "The teachers know they are accountable. They want to do what they are supposed to do and impress him, have good test scores and for the students to do well" (CG, 1/8). Terry Peroni at D.A. Malone described Betsy Master's effect on teachers:

Betsy is a role model. She has high standards and high expectations. If you have high standards, people will try to measure up to those standards, just like [the high standards] we have for our students. ... We want to make sure we raise everybody up. ... We're doing what we need to do to help students grow and be productive citizens. (TP, 3/3)

\section{Feeling Valued and Affirmed as People and Professionals}

The strong interpersonal disposition of Edmonds, Rippy, and Kendall and their efforts to nurture positive relationships with teachers and treat them with dignity and respect lead teachers to report feelings of being valued and affirmed, both as people and in their work as professionals. Daniel Baker of Cane Ridge Elementary found this a unique feature of Marie Edmonds's leadership: “I'm speaking about as far as values and behaviors and respect — that's not across the board every place [other schools] you go" (DB, 11/20). "I think it's very important to feel that you are appreciated," Jenna Wilson said of Donna Rippy (JW, 1/30). Teacher Katie Turner appreciated Bill Kendall's concern for her as a human being: "I know he cares about Katie Turner the person, 
probably more than he cares about the job that Katie Turner the teacher is going to do" $(\mathrm{KT}, 1 / 8)$.

\section{Strong Affiliation with School and Learning Climate}

Because the teachers felt valued and affirmed, and connected these perceptions to Edmonds, Rippy, and Kendall's theories of practice, the teachers also experienced a strong affiliation with their schools. They valued the positive climate the principals fostered and did not entertain thoughts of working elsewhere. "We are happy to be here, thankful to be in this working situation because we know full well not all schools operate the same way," Daniel Baker said of Marie Edmonds's effects on teachers at Cane Ridge (DB, 11/20). Julie Jones of Cane Ridge agreed: "We are like a family" (JJ, 10/16).

Helen Bowen expressed similar perceptions of Donna Rippy's leadership at Cherrywood: "I feel really good about being here. I think she holds us in high regard. I love working here" $(\mathrm{HB}, 1 / 25)$. Katie Turner endured a long commute in order to work at Homestead with Bill Kendall: "I drive almost 45 minutes a day to be here because I love being here. There's several other schools much closer to my home and I have friends and family who think, 'Can you not get a job there?' But I don't want to leave.” Turner also valued being at Homestead because she wanted that school environment for her own daughter. "We're in a school where she's loved and she's cared about and she knows that. And that's how we feel as teachers, and that leads us to work even harder" $(\mathrm{KT}, 1 / 8)$.

\section{Believing Their Opinions are Valued}

Teachers described how Edmonds, Rippy, and Master's efforts to invite teacher input made them feel valued as professionals. Daniel Baker reported that Edmonds 
sometimes provided teachers an overall goal, but gave them wide leeway and a voice in determining how the goal was reached (DB, 11/20). Darla Hammond agreed: "Teachers feel important, that what they are doing and what they know is important. Our teachers greatly admire Ms. Edmonds and to have her value their opinion is a treasure. She gets buy-in and ownership when she values teachers" (DH, 3/8). "Teachers are motivated by the feeling of being a part of what takes place in our school," Steve Yeager said of Donna Rippy's efforts to involve teachers in decision making (SY, 2/26). Terry Peroni described the effects of Betsy Master's theory of practice for inviting teacher input: "Ms. Master really wants to hear our opinions about situations and she makes everybody feel very comfortable with different ideas, even if they're different from her ideas or her perspective" (TP, 3/3).

Desire to Meet Principal's Expectations

Teachers at Edmonds, Rippy, and Kendall's schools responded to their theories of practice by expressing a desire to meet the principal's expectations. Teachers at Cane Ridge were motivated to improve student achievement because they respected Edmonds's efforts and wanted to meet her standards. According to teacher Daniel Baker, "I don't want her to be disappointed. I want to meet the expectations she has for me" (DB, 11/20). Darla Hammond agreed:

Even though Ms. Edmonds isn't going to be on your back constantly going, "Are you using this? Are you doing this?" you know what her standards are and you respect her because of that, so you want to live up to those standards. You are constantly asking yourself, "Am I doing all I can? Am I doing what Ms.

Edmonds wants?” (DH, 10/16)

Steve Yeager expressed similar perceptions of Donna Rippy's personal concern for teacher well-being: "I think her caring about everybody makes them want to work 
harder for her at times, even when it may not be the best day" (SY, 2/1). Carrie Gergan reported that teachers valued Bill Kendall's view of their work: "They want to do what they are supposed to do and impress him, have good test scores and for the students to do well" (CG, 1/8).

\section{High Levels of Job Satisfaction and Motivation}

Teachers at three case study schools reported high levels of job satisfaction and motivation. This effect was sometimes connected to other effects. For example, because teachers felt valued and affirmed, they were consequently motivated to work hard and take responsibility for student success. "I can’t really imagine having a better working situation," Daniel Baker of Cane Ridge reported. "I feel valued here" (DB, 11/20). Steve Yeager described Donna Rippy's effects on teachers at Cherrywood:

You want to come to work when you feel good. You always have your good days, bad days, you even sometimes go through a funk every once and a while where you may have a month when you feel, "Oh, I just can't do another day." But if you feel like, if there's any place I've got to go to work, this is the one place I want to go, that helps you get up in the morning and get there. You feel more of a dedication too when you work for somebody that you know cares about you more than just ... as an employee. (SY, 2/1)

Sherry Gann of Homestead Elementary believed that Bill Kendall's positive relationships with teachers contributed to a strong learning environment that ultimately led to higher student achievement. "The teachers feel good, so the students are going to feel good:"

That's why there's such a good learning environment here.... When you are in an environment that you feel stressed or you constantly feel like you are being watched and you're going to be hammered on, you're going to find it a really tense situation for teachers because they are all going to be complaining. I just don't hear much complaining here. (SG, 1/8) 


\section{Other Effects}

Some effects were reported at only two of the four schools.

Actively engaged in collaboration. Because of the heavy emphasis Edmonds and Rippy placed on collaboration, teachers reported high levels of teamwork and collaboration at their schools. Observations at both schools revealed teachers meeting regularly to share instructional ideas, partner to solve problems and develop interventions for students, and make decisions.

Embraced new ideas and continuous growth. While three principals emphasized continual professional growth, teachers at Edmonds and Master's schools reported a strong sense of responsibility and acceptance of on-going professional learning as a key effect of the principals' theories of practice. Even veteran teachers like Marie Vintner of D.A. Malone, who described herself as being from the "Stone Age," said she embraced "improving all the time. I'm all the time trying to be better" (MV, 2/22). Teachers "really do attempt to do the new things [Master] introduces and most will follow through and continue to do them long after," according to Amy Seton (AS, 3/3).

To summarize the results of Research Question 2, teachers at the case study schools reported eight key effects of principal theories of practice, including the following: (a) teachers experienced a personal sense of responsibility for student learning outcomes, (b) felt valued and affirmed as professionals and people, (c) had a strong affiliation with the school and a positive view of the learning climate, (d) believed their opinions were valued, (e) desired to meet the principal's expectations, (f) experienced high levels of job satisfaction and motivation, (g) actively engaged in collaboration and teamwork, and (h) embraced new ideas and continuous professional growth. The next 
section explores Research Question 3, dealing with double-loop learning and reflectionin-action.

\section{Reflective Practice in the Case Study Principals (Research Question 3)}

Research Question 3 investigated the extent to which the case study principals used double-loop learning as described by Argyris and Schön (1974) or the more broadly defined "reflection-in-action" described in Schön's (1983) conception of the "reflective practitioner." Double-loop learning is the cognitive process by which a practitioner uses feedback to reflect on his or her theory of action, and then questions and revises the underlying assumptions rather than simply adjusting action strategies to achieve a new outcome. Schön elaborated on the idea of double loop learning by describing the process by which a professional approaches problems in a reflective manner. This "reflection-inaction" included the following steps: a) recognition of the problem; (b) recognition of incongruities; (c) evidence of reframing of the problem; (d) generation of new solutions; (e) testing-in-action of solutions; and (f) evaluation of outcomes.

While there were many examples of principals altering their action strategies to achieve different outcomes (the more common "single-loop learning"), case study principals struggled to identify instances in which they had actively questioned their own underlying assumptions, or experimented with new action strategies based on revised assumptions. The principals did, however, report largely congruent areas where they had developed "special case" theories of practice to accommodate situations in which their preferred theories of practice did not work. Specifically, three of the four principals described how they developed more directive and corrective techniques for giving teacher feedback when their preferred methods of leadership failed to improve teacher 
effectiveness. Three of four principals also described how they had learned to be intentional about inviting teacher input into decision-making. This heightened emphasis on soliciting teacher feedback and input did not constitute new assumptions about leadership, but rather represented a kind of single-loop learning in which the principals opted to place more emphasis on a particular action strategy in order to enhance teacher job satisfaction, and thereby promote higher levels of student achievement (see Table 4). Each of these examples of reflective practice is described below.

Table 4

Changes in Leadership Suggesting Reflective Practice

\begin{tabular}{lcccc} 
Change & Edmonds & Rippy & Kendall & Master \\
\hline Gave more directive feedback & $\mathrm{X}$ & $\mathrm{X}$ & $\mathrm{X}$ & \\
Invited more teacher input & & $\mathrm{X}$ & $\mathrm{X}$ & $\mathrm{X}$ \\
\hline
\end{tabular}

Note. $\mathrm{X}=$ Change emerged in this case study.

\section{Giving More Directive Feedback}

Edmonds, Rippy, and Kendall were all highly interpersonal in their approach to leadership. These three principals all used theories of practice designed to nurture positive personal relationships with staff. These theories of practice were based both on assumptions that all people possess inherent worth and should be treated with dignity, compassion, and respect, and that teachers who felt valued and affirmed would experience higher levels of job satisfaction, motivation and effectiveness. Because of this orientation toward the emotions of others, these principals preferred positive, affirming forms of leadership, including modeling and offering suggestions, praise and 
encouragement. The principals discovered, however, that not all teachers responded to these non-directive action strategies. Some teachers were not effective, or struggled with instructional and classroom management deficiencies and failed to improve their performance when the principal used his or her preferred methods. As a result, Edmonds, Rippy, and Kendall had developed more directive approaches, including giving corrective feedback, placing teachers on improvement plans, and non-renewing untenured teachers who were underperforming. As many of the teacher informants confirmed, using these "special case" theories of practice was not easy because of the interpersonal tension and difficult emotions involved, but the principals had grown more confident over the years in using such directive forms of leadership. "He doesn't want to hurt anybody's feelings," Ms. Gann said, describing Bill Kendall, "but yet, he does make it clear to everyone what is expected" (SG, 1/8). While their core assumptions about what constituted effective leadership did not change, these principals did develop new assumptions about how to handle situations when their preferred theories of practice were not effective, and in this sense, exhibited evidence of "double loop learning."

Darla Hammond described the change in Marie Edmonds:

She's had to get tougher. It's not in her to be the tough leader and to have to go to people and say, "No, you've got to do it like this." I think she's seeing that some people don't get it just by example or by whole group instruction. Or maybe they don't understand quite how to implement it, so she's had to become a little harder, a little more personal, one-on-one, with, "You need to do this." She'd much rather lead by example, by modeling than by being that tough. Different people have different personalities and some people just don't get it that way. (DH, 10/16)

Donna Rippy confirmed that using corrective feedback was not pleasant for her, and she had been reluctant to use it in the early years of her principalship. "I'm not one to upset the apple cart," she explained. In recounting her decision to not renew the 
contract of an untenured teacher the previous year, she acknowledged that earlier in her career, she "would not have handled it," and she expressed regret that she didn't confront the problem sooner $(\mathrm{DR}, 12 / 20)$.

During the reflective exercise in which the principal recalled a post-observation conference with an underperforming teacher, Bill Kendall, like Edmonds and Rippy, expressed regret that he was not more directive with the teacher in question:

If I had to deal with him today, I would probably have been a lot more forceful with him from the beginning instead of giving him time to shape up. I just kept hoping he was going to improve, but he never did. I would've been more directive and forceful in that this is my expectation and you will meet it. (BK, $1 / 15)$

The principals expressed more confidence in using corrective feedback because they knew their most basic responsibility was to the students and to the learning climate they were trying to create. "You're not doing anybody a favor by keeping them in a job where they're going to be miserable and make your life miserable," Kendall said of underperforming teachers. "You're not helping them or yourself and you're certainly not helping the kids" (BK, 12/11).

The "special case" theory of practice for giving corrective feedback emerged from principal reflection on their failures to address teacher improvement needs with their preferred methods. Principals then assumed that when more positive, affirming forms of feedback failed to bring changes in teacher behavior, a new action strategy was needed. This process of reflecting on their failures and adjusting their theories of practice accordingly appears to be a form of double-loop learning. 


\section{Inviting More Teacher Input}

While inviting teacher input emerged as a theory of practice for all case study principals, Rippy, Kendall, and Master described how they had improved in their use of this theory of practice over the years. All three acknowledged that while they never assumed that inviting teacher input was unimportant, events during their principalship had taught them that not emphasizing the attendant action strategies of soliciting teacher feedback and actively engaging teachers in decision making could lead to negative results. Conversely, inviting teacher input paid major dividends in terms of teacher job satisfaction, buy-in, and support of school wide initiatives. Rippy, Kendall, and Master all reported using this approach as one of their key instructional leadership theories of practice.

Donna Rippy discussed how difficult it was for her to share power with teachers when she first became principal at Cherrywood Elementary: "When you are first entrusted with this job, you think you have to know everything. Therefore, you think you have to have your hands in everything." She explained her perceptions as a lack of confidence in herself, not an assumption that she could not rely on teacher input. "I knew from day one that the teachers had it together, but I didn't" (DR, 1/22). As she became more confident in her abilities to be effective as principal, Rippy paradoxically grew more comfortable sharing her power with teachers:

As you learn your people and move through different experiences you grow, and I think that's what happened to me. I realize that I don't have to have my hand in everything. I can let the teachers run with it. It never felt good when I tried to be the one in charge. (DR, 2/4). 
Bill Kendall echoed Rippy when he described his own efforts to invite more teacher input, which did not constitute a change of assumptions, but rather a refinement of emphasis:

You get more bees with honey. I don't know that I use more honey these days, but I think I talk to them more before making a decisions. I get their opinions. Not that their opinions didn't matter before, but I think now I trust their opinions. ... When I started I didn't ask anybody anything. It wasn't that I learned that I better do it, I just decided it worked best for me. In the beginning, I didn't know who to get input from. As I got to know people more, I knew which people I should talk to ... I make better decisions based on my conversations with people and thinking things through instead of making an off-the-cuff decision. I have grown wiser to taking my time. I used to think I had to make my mind up right now. I don't anymore. It's alright to wait. (BK, 12/16)

Betsy Master described the incident that "blew up and bit me in the face" as a turning point in her intentional use of teacher input as an instructional leadership action strategy. Master claimed she had never resisted teacher input, but after a backlash from teachers over her unilateral decision regarding the assignment of language arts classes led to a painful division in the faculty and the intervention of the superintendent, she took a harder look at her practices:

In retrospect, that was one of the things that helped me change and grow because I did go to the superintendent-well, I was actually brought to the superintendent, and we did talk about it and I did get some input from other administrators who saw things differently and could see more objectively than I did. I made some changes in how I did things. So although at the time, going through that, it wasn't the most pleasant thing, but it was an opportunity for me to grow and learn. (BK, 2/19)

Master credited the advice of her superintendent as a source of new understanding

for how to improve her effectiveness:

What I was asked to look at was, "Are you communicating at the level you think you are? Maybe we need to be looking at some different ways to do that and take some of the things that were causing a little bit of turmoil and be more proactive." (BK, 2/19) 
As a result, Master made intentional efforts to communicate with staff and involve teachers in key decisions.

While the changes in principal theories of practice regarding teacher input did not appear to exhibit double-loop learning in that their core assumptions remained the same, the principals nevertheless recognized the incongruence between their actions and desired outcomes, and made tentative adjustments in their action strategies which proved to be more effective over time. In this sense, the principals displayed behaviors consistent with the idea of the "Reflective Practitioner."

\section{Linkages to Previous Literature}

This study's results are consistent with previous research indicating that effective principals influence student achievement by their impact on school culture and climate variables. The case study principals focused their instructional leadership efforts on productive interactions with teachers. Their theories of practice featured action strategies that paralleled behaviors proven in earlier literature to impact teachers in positive ways. This study shed new light on effective principal behavior, however, by using the theory of practice framework, which identifies not only actions but also the underlying assumptions that shape those actions and their intended effects. The results make an important contribution to literature on theories of practice by applying the concept to the work of successful school principals and revealing new insights into the extent to which principals reflect on their professional practice.

\section{Principal Effects on Student Achievement}

Many previous studies explored the indirect effect of principal leadership on student achievement (Heck, 1993; Heck, et al., 1990; Pounder, et al., 1995; Snyder \& 
Ebmeir, 1993; Witziers, et al., 2003). Heck, et al. (1990) found that a principal's approach to school governance affected school climate and instructional organization, and that together governance, climate and instructional organization impacted student achievement. Heck et al. concluded that principals in high-performing schools actively involved teachers in instructional decision-making, and exhibited other traits such as (a) expressing and enforcing high expectations for student academic and social behavior, (b) establishing clear school-wide goals, (c) encouraging the study of instructional strategies, and (d) encouraging faculty enthusiasm and good morale. Likewise, Heck (1993) found that principals in high-achieving schools actively involved teachers in instructional decision-making. Snyder and Ebmeir (1993) and Pounder, et al. (1995) further confirmed that principal effects on student achievement were mediated indirectly through such teacher-level variables.

Principals in the present study used theories of practice congruent with the findings of these previous studies. All four principals held theories of practice for inviting teacher input into school-wide decision-making. Edmonds, Kendall, and Master all used theories of practice for encouraging ongoing professional growth focused primarily on the use of new instructional strategies. An underlying assumption or action strategy related to high expectations for all students, and the relationship orientation of the principals facilitated high levels of teacher enthusiasm and morale. In this sense, the present study further confirmed previous findings.

In their meta-analysis of previous studies on principal effects, Witziers, et al. (2003) found that four specific leadership behaviors significantly affected student achievement: (a) supervision and evaluation, (b) monitoring, (c) visibility, and (d) 
defining and communicating mission. The present study partially confirmed these results. Three of the four principals used theories of practice for providing feedback, including corrective and directive feedback when necessary, to improve teacher performance, behaviors analogous to what Witziers et al. called "supervision and evaluation.” Betsy Master of D. A. Malone Middle School emphasized action strategies such as requiring careful documentation of teacher improvement efforts and using the formal evaluation process, behaviors included both under "supervision and evaluation" and "monitoring." Finally, like the subjects of studies reviewed by Witzier et al., the participant principals in this study all emphasized a common mission, though each principal maintained a slightly different focus. For Marie Edmonds, the mission centered on meeting the unique learning needs of each individual child. For Donna Rippy, the mission focused on preparing elementary students for a rigorous secondary school curriculum. Bill Kendall emphasized the professional duty to carry out state and district mandates. Finally, Betsy Master stressed the common goal of continuous teacher improvement. All principals unified staff around a core mission, and each school's culture reflected the principal's sense of mission.

\section{Principal Effects on Teachers}

Based on this indirect connection between principal behaviors and student achievement, another stream of educational research documented the effects of principal behaviors on teachers (Blase \& Blase, 1994, 1999; Blase \& Kirby, 2000; Blase, Blase, Anderson, \& Dungan, 1995; Freidkin \& Slater, 1994; Short, 1994). Hallinger, et al. (1996) showed how positive effects on teachers were directly correlated with higher levels of student achievement. 
Friedkin and Slater (1994) found a significant association between a principal's centrality to the advice network within the school and student performance. The extent to which teachers looked to the principal for instructional advice and support impacted student outcomes. In the present study, principals used theories of practice for promoting on-going teacher professional growth, innovation, and the use of new instructional strategies. As a result, teachers in the case study schools looked to their principals for leadership on these topics and engaged in continuous professional learning. Marie Edmonds, in Case Study A, understood collaboration to be the most effective means of promoting on-going growth in teacher instructional knowledge. Her theory of practice and those of the other principals regarding collaboration and the resulting levels of teacher teamwork and collaboration paralleled Short's (1994) findings. Short discovered that in schools with effective teacher teams, principals played a critical role by encouraging and facilitating teacher reflection through stimulating teacher collaboration and problem solving.

In addition to promoting high levels of teacher learning and collaboration, principal theories of practice in the present study influenced teachers in a variety of other ways, including fostering high levels of teacher job satisfaction and motivation, a sense of teacher autonomy and responsibility, and a strong affiliation with the school and with the faculty as a community and team. These results were also consistent with previous research on principal effects on teachers. In particular, this study echoes a series of studies focusing on principals known for promoting teacher empowerment and involvement in self-governance (Blase \& Blase, 1994, 1999; Blase, et al., 1995, Keedy \& Finch, 1994). 
Blase and Blase (1994) discovered that shared-governance principals affected three dimensions of teacher empowerment: (a) the affective dimension (teacher satisfaction, motivation, esteem, confidence, security, sense of inclusion, and identification with colleagues as a unified group); (b) the classroom dimension (including innovation, creativity, reflection, autonomy, individualization of instruction, professional growth and classroom efficacy); and (c) the school-wide dimension (expression, ownership, commitment, sense of team, and school-wide efficacy). These results were further confirmed by Blase, et al. (1994) through in-depth interviews with the same principals, and in Keedy and Finch's (1994) case study of a principal's efforts to share power with teachers. The present study suggests that the theories of practice used by the case study principals employed assumptions and action strategies with a wide range of desirable effects on teachers, consistent with the findings of Blase and Blase (1994), Blase et al. (1995), and Keedy and Finch (1994).

Blase and Blase (1999) elaborated on the findings of their previous studies by developing the Reflective Growth Model (RGM) of instructional leadership. The action strategies embedded in principal theories of practice revealed in the present study parallel the RGM in numerous ways. The RGM consisted of two overarching themes of effective instructional leadership. The first theme involved a set of behaviors the researchers categorized as "talking with teachers to promote reflection," which included (a) making suggestions, (b) giving feedback, (c) modeling, (d) using inquiry to solicit advice and opinions, and (e) giving praise.

The second category of behaviors involved "promoting professional growth," and included (a) emphasizing the study of teaching and learning, (b) supporting collaboration 
efforts among teachers, (c) developing coaching relationships among educators, (d) encouraging and supporting the redesign of programs, (e) applying the principals of adult learning, growth and development to all stages of staff development, and (f) implementing action research to inform instructional decision-making. All of these behaviors were embedded in the principal theories of practice in the present study.

Likewise, the effects on teachers Blase and Blase (1999) associated with the Reflective Growth Model also parallel the findings of the present study. Blase and Blase found that principal behaviors of the RGM impacted teacher attitudes toward instruction in a number of ways: increasing teacher levels of motivation, self-esteem, efficacy, feelings of support, sense of security, reflection and willingness to engage in innovation, creativity and risk-taking. The present study supports the structure of the Reflective Growth Model of instructional leadership, and further clarifies the model by elaborating on the assumptions that guide the action strategies of effective, empowering principals.

\section{Change Resistance in Schools}

Cusick (1992) reviewed descriptive studies from three decades of research on school culture and found an overwhelming orientation toward control and bureaucratization. Such an orientation among teachers and principals is not likely to foster the collaborative relationships Keedy and Achilles (1997) expected to find in a genuinely "restructured" school. The principals in the present study exhibited theories of practices that were markedly atypical of the schools Cusick described. The case study principals placed a high value on personal relationships of trust, openness and generosity. Key theories of practice for Edmonds, Rippy, and Kendall focused on nurturing personal relationships and greatly shaped the principals' instructional leadership. Because 
principals trusted teachers and respected their personal and professional wisdom, they encouraged teacher autonomy, teacher input into decision making, and teacher collaboration.

Such approaches involve a surrender of control on the part of principals, and a willingness to take risks. Case study principals spoke openly of their desire to share power, or their conviction that they would not be effective leaders if they made all decisions or were not open to teacher input. "I look at myself as the leader of the building, but I don't look at myself as being all knowing,” Donna Rippy explained (DR, 1/22). "Teachers know the students better than I do," Bill Kendall shared. "I ask their opinion and we try to do things by consensus whenever we can" (BK, 12/11).

Keedy and Achilles (1997) described how structural reforms had failed to alter the basic relationships and norms of behavior of school personnel. They imagined a "restructured" school as one that builds capacity for change through relationships marked by “compassion, cooperative effort, student mindfulness and mutual respect for articulate and diverse positions on crucial issues" (p. 3). Principals and teachers in such schools would use more collaborative approaches to decision-making and support self-inquiry and self-development. The risks inherent in an environment open to inquiry and innovation would be offset by the advantages of more open flows of communication.

The principals in the present study used theories of practice and fostered school climates consistent with those imagined by Keedy and Achilles and responded to the attendant risks by reflecting more deeply on their practice. Marie Edmonds, for example, found that encouraging teacher input and collaboration encouraged a level of teacher empowerment that sometimes frustrated her own legitimate use of principal authority. 
Betsy Master discovered that not being careful about involving teachers in decisionmaking when they have come to expect involvement could lead to a climate-damaging backlash. These results led both principals to think carefully about their assumptions and action strategies and to more carefully and intentionally communicate with teachers and solicit their input.

Also consistent with Keedy and Achilles's recommendations, the principals intentionally built compassionate personal relationships with teachers, and used affirming, positive leadership strategies like modeling, praise and making suggestions to support those relationships. The principals conversely avoided more corrective forms of feedback and directive forms of leadership until their action strategies failed to promote instructional effectiveness for some teachers. As a result, the principals reflected on their assumptions and action strategies and developed special case theories of practice to achieve the desired effect.

So principals in this study provide examples of leadership that contradict the traditional, ossified modes of thinking and action described by Cusick (1992). Keedy and Achilles (1997) imagined that the use of joint principal and teacher theories of practice could build the capacity for meaningful school reform. The case study principals did indeed use theories of practice that embodied the kinds of relationships Keedy and Achilles suggested, and did engage in some degree of reflection on their practice.

Sirotnik (1989) argued that such self-reflection and relationships of trust would be hallmarks of schools that are striving to become "centers of inquiry" rather than "targets of reform," schools with a real capacity for impacting student achievement. When schools become centers of inquiry, collaborative reflection becomes a guiding principle 
of professional practice, in which teachers and principals engage in an ongoing process of inquiry, reflection, testing of new hypotheses and approaches, evaluation of effects, and further inquiry.

The case study principals fostered the requisite relationships of a restructured school, and promoted environments of collaboration and professional growth. Teachers responded to their theories of practice with high levels of motivation and teamwork and exhibited personal responsibility for student outcomes and continuous learning. There was limited evidence in the data, however, to conclude that the schools had become centers of inquiry in a way that was self-conscious to the principals and teachers themselves.

\section{Theories of Practice}

This study makes a contribution to research literature on theories of practice by mapping the cognitive structure and effects of successful school principals' theories of practice of instructional leadership. Argyris and Schön (1974) first articulated the concept of theories of practice as a model for exploring the behaviors of professionals in practice situations. Argyris and Schön attempted to link behavior with the powerful, underlying assumptions professionals brought to problems of practice in a way that could promote intentional self-reflection and improvement of professional effectiveness. Lipshitz (2000) argued, however, that despite the widespread use of Argyris and Schön's ideas in discussions of organizational learning, relatively few empirical studies have explored the usefulness of the theory of practice concept for understanding and evaluating the actual behaviors of professionals. The present study responded to 
Lipshitz's call for more research, contributing to a small but growing body of literature on theories of practice.

In seeming contradiction to Argyris and Schon's assumptions about most professional practitioners, Tsangaridou and O'Sullivan (200) found a high level of congruence between physical education teachers' espoused theories regarding instructional practice and their theories in use. The present study also revealed a high degree of consistency between the espoused theories of case study principals and their actual theories of practice. This consistency was supported through interviews with experienced teachers and naturalistic observations. To the extent that reflective practitioners exhibit more consistency between espoused theories and theories in use, data from this study provides further linkages between reflective practice and higher levels of student achievement.

\section{Assessment of Results}

As previously discussed, the school principal behaviors described in this study are consistent with past research exploring how principals can influence student achievement indirectly through creating empowering, collaborative, and emotionally supportive school cultures and by holding teachers accountable to a common, student-centered mission and continuous professional improvement. In today's accountability culture, shaped by NCLB and other state-level mandates, this study reinforces the critical importance of the principal-teacher relationship to student outcomes. The unique contribution of this study, however, is its use of the theory of practice framework, which not only reveals the behavior and effects of successful school principals, but also their underlying assumptions of professional practice, and their use of self-reflection as a means of 
refining and enhancing their practice. The results suggest a variety of implications for the deeper uses of the theory of practice framework for improving principal effectiveness, as well as principal recruitment, training, and professional development.

Argyris and Schön (1974) argued that organizational learning is often impeded by the unstated and sometimes unconscious values, beliefs and assumptions members of the organization bring to their work. In mapping the cognitive structure of successful principal theories of practice for instructional leadership, this study revealed the core assumptions that shaped the principals' action strategies. Specifically, the principals held assumptions about the importance of collaborative leadership and continuous learning that were rooted in both moral and utilitarian perspectives. Principals wanted to nurture positive personal relationships because they believed in the dignity and inherent worth of teachers as individuals and professionals. Based on this appreciation for the individual, the principals believed the contributions of individuals enriched the collective wisdom and effectiveness of school as a unit. Therefore, principals engaged in efforts to promote teacher collaboration and empower teachers by inviting their input, giving them autonomy, and supporting their ongoing professional growth. Ultimately, principal action strategies emerged from these assumptions with the desired effect that student achievement would increase. Findings from this study suggest that these specific instructional leadership assumptions were indeed linked to higher levels of student achievement, as evidenced by the success of these schools.

Argyris and Schön made a careful distinction between assumptions and action strategies that hindered reflective practice (Model I theories of action) and those that supported reflective practice (Model II theories of action). Model I involves the 
expectation that most problems present win-lose outcomes for individuals. These assumptions lead to action strategies for controlling the external circumstances of a situation and avoiding the vulnerability of making one's feelings and internal motivations known to others. Defensive behavior that reinforces the individual's underlying assumptions is the primary result of Model I. Argyris, Putnam, and Smith (1985) argued that Model I theories of action discourage inquiry and promote defensive relationships, result in low freedom of choice, reduce production of valid information and provide little public testing of ideas. Model II behavior, by contrast, encourages double-loop learning, inquiry and questioning of values and assumptions, and the deliberate identification and testing of theories in use. In an organizational context, Model II typically involves assumptions that value shared leadership, open, dialogical processes of problem-solving and participatory decision-making. Data from the present study reveal principal theories of practice that emerge primarily from Model II assumptions and action strategies: trusting relationships, collaboration, shared decision-making and improvements in professional practice. Thus, a further implication of this study is that principal instructional leadership theories of practice rooted in Model II assumptions also contribute to higher levels of student outcomes, given the success of the case study schools.

The point of Argyris and Schön's (1974) argument, however, is that Model II assumptions and action strategies are not just effective in achieving positive outcomes, but contribute to reflective practice, including double-loop learning. The principals in this study exhibited some signs of reflective practice, but the results were limited. There was only one consistent example within the data of double-loop learning: three of the 
four principals had developed special case theories of practice for providing corrective feedback when their preferred methods of modeling, providing praise, and offering suggestions failed to correct problems in teacher performance. Within the broader concept of "reflective practice," which does not necessarily imply a revision of assumptions, the principals again exhibited only one example: they had learned that their professional effectiveness was enhanced - and serious school culture problems were avoided - when they actively communicated with teachers about instructional decisions and solicited their feedback and input.

Why did these principals fail to exhibit more evidence of reflection-in-action, when their theories of practice clearly contributed to positive teacher outcomes, and likely contributed to higher levels of student achievement, and when their assumptions and action strategies mostly conformed to Model II theories of action as defined by Argyris and Schön?

Moreover, while there were high levels of collaboration and a general acceptance of ongoing professional learning in the case study schools, there was little evidence that they had become "centers of inquiry" (Sirotnik, 1989). The conceptual framework for this study suggested that if principals engage in reflective practice, they can reshape the core relationships in their schools in such a way that a genuine capacity for improvement is born. Eventually teachers and principals will together engage in a dynamic process of reflecting on and questioning their assumptions and action strategies, testing new theories of practice, evaluating the results, and beginning collaborative inquiry again, in a neverending creative cycle of continual growth and learning. The schools in this study were remarkably successful both in terms of student achievement on the state assessment and 
their positive climate. Why was there little evidence of such a dynamic process of experimentation, inquiry, exploration, and reflection?

These questions point the way toward further study of principal theories of practice. Data from this study, however, suggests some possible explanations. First, while many of the principal assumptions and action strategies followed the Model II form, the principals' strong concern for the emotions and well-being of others, manifest in the common theory of practice for nurturing positive personal relationships, may have actually taken a Model I form. According to Argyris and Schön (1974), Model I assumptions and behaviors often include a strong inclination to avoid interpersonal conflict, including protecting the feelings of others or avoiding discomfort for oneself. All four principals acknowledged their difficulty and reluctance in causing emotional harm to teachers by correcting their performance problems, especially in the early years of their principalship. Bill Kendall exemplified the principals' perceptions when he said, "[It's] not easy when you've got a teacher sitting here and it's her first year teaching and tears are rolling and she's paid all this money to go to college and get a job as a teacher because she thinks that's what she wanted to do" $(\mathrm{BK}, 12 / 11)$. While the principals grew more confident in giving corrective feedback, they admitted they had been slow to use it in the past and still found doing so difficult. Donna Rippy expressed regret for allowing some underperforming teachers to get tenure because she was uncomfortable correcting their behaviors (DR, 1/22). Perhaps this reluctance to confront poor teacher performance contributed limitations to the school's collective capacity for deep, collaborative reflection on their practice, both because of the emotional discomfort it might invoke, and 
because weak teachers remained on staff who were perhaps incapable of this level of thoughtful reflection-in-action.

Another explanation may lie in the training and preparation of principals for their work as school leaders, and in the expectations and daily routines that shape principals' work. Principal participants commented on how refreshing the process of discussing and reflecting on their work was (ME, 12/4; DR, 3/12). None of the four principals was familiar with the theory of practice concept or the notion of reflective practice prior to their participation in this study. Principal preparation programs tend to focus on the technical knowledge future administrators will need to manage their buildings effectively, such as school finance procedures, special educational law, and administration of personnel. Relatively little time is devoted to helping aspiring administrators reflect on their assumptions or theories of action (Keedy, 2005). These technical issues remain the focus of most professional development for practicing principals. Likewise, much of the principal's day centers on managerial issues such as scheduling, student discipline and paperwork, rather than actual instructional leadership (Cusick, 1992). As a result, most principals, including the participant principals in this study, received no training for nor have the opportunity as their daily activities unfold, to engage in reflective practice. If principals are not reflective about their own practice, how can they guide teachers in the process of theory development, testing and evaluation that would characterize a school as a "center of inquiry?"

Finally, state and federal education policy defines the single standard of school success as a steady increase in student test scores. The selection criteria for principals who participated in this study included continuous improvements in student achievement 
as measured by Kentucky's Commonwealth Accountability Testing System (CATS). This relatively narrow definition of what constitutes a successful school may provide significant limitations to the reflective practice of teachers and principals. When student test scores are the lone standard of success, the range of topics relevant to reflective professional practice may be dramatically limited. While schools featured in the present study were marked by high levels of collaboration among teachers, if the focus of such collaboration centered solely on those activities that would lead to higher student test scores, other areas of collective inquiry and reflection may be neglected. Likewise, principals in this study strongly emphasized continuous teacher professional growth. The chief vehicle for teacher learning was the Thoughtful Classroom initiative, based on the research of Robert Marzano and his conclusions about instructional strategies that are proven by research to be successful. Sirotnik (1989) argued for a kind of collaborative inquiry that was deeply rooted in the contextualized experiences of teachers and their students. If teachers studied these strategies — collaboratively or alone — with little reflection or testing as to the efficacy of these strategies for their particular students and their particular school, then little reflective practice may have taken place.

Suggestions for Education Stakeholders and Researchers

Findings from this study offer a variety of implications for principal preparation programs, district recruitment efforts, principals and others interested in meaningful professional development, and finally for researchers .

Suggestions for Principal Preparation Programs University principal preparation programs should review their admissions procedures to recruit future administrators with a stronger orientation toward self- 
reflection. Keedy (2005), Keedy and Grandy (1999), Levine (2005), and Creighton and Jones (2001) have criticized the low admissions requirements and quality of principal preparation programs nationwide. As one component of improvement efforts, preparation programs should ask applicants to provide examples of reflective practice and double-loop learning in their previous work, and admit students with a demonstrated capacity for reflective practice. Likewise, programs should restructure curricula to preserve the technical knowledge future administrators need to be effective while enhancing student opportunities to study all components of the theory of practice framework, including discovering their own theories in use, and experimenting with the development, testing, and evaluation of new theories of practice rooted in actual problems principals face in the field.

\section{Suggestions for School Districts}

Like university principal preparation programs, school districts recruiting, interviewing and hiring new principals should consider applicant capacity for reflective practice, and should carefully explore the assumptions that guide applicants' theories of action. This study makes a link between certain principal assumptions and positive teacher outcomes that previous research associates with higher levels of student achievement. As school districts strive to meet state and federal mandates for improving student performance, district leaders should pay careful attention both to principal assumptions of instructional leadership, and to their willingness and capacity to reflect on their practice and adjust assumptions and action strategies based on contextualized feedback from teachers and students. 


\section{Suggestions for Principal Professional Development}

Individual principals, school districts, and education policy makers should use the concept of theories of practice to revitalize principal professional development and school-wide improvement efforts. Professional development that focuses exclusively on principal technical knowledge simply reinforces the theory-practice divide that impedes deep forms of professional self-reflection and improvement (Keedy, 2005). Erlandson (1994) argued that especially as principals advance in years of experience, their need for technical knowledge lessens and their desire for opportunities to reflect thoughtfully on their practice increases.

Moreover, previous research shows that simple structural changes within schools, such as changing governance structures, or mandating out-of-the-box professional development or whole school reform programs, has little lasting impact on school culture or student achievement (Datnow, 2003; Hargreaves \& Goodson, 2005; Keedy \& Achilles, 1997). Theories of practice and reflection-in-action provide vehicles by which principals and teachers can reflect collaboratively on their collective assumptions and action strategies for managing instruction, and experiment in whole-school inquiry into the revision, testing and evaluation of alternate theories of action to improve student outcomes. Universities and school districts should develop partnerships that assist practicing principals and teachers in crafting context-specific opportunities for learning the theory of practice framework and collaboratively engaging in these forms of collective self-reflection. Such efforts would move schools much closer toward Sirotnik's (1989) vision of schools as "centers of inquiry" rather than mere "targets of change." 


\section{Suggestions for Researchers}

This study contributes to the literature on theories of practice, instructional leadership, and school principal effects on teachers. The results suggest a number of important directions for future research studies. Research questions limited this study to documenting the instructional leadership theories of practice of successful principals, their effects on teachers, and the extent to which the principals were reflective about their practice. Future studies should explore other dimensions of the theory of practice framework in greater depth, including the congruence between espoused theories and actual theories in use. Data demonstrated that principals in this study had little if any gap between their espoused theories and theories in use. Further research should explore whether this is typical of successful principals in a wide variety of contexts, and if there are differences among principals regarding this gap, what variables account for that difference. Likewise, research questions for this study did not specifically seek to categorize principal theories of practice according to the Model I-Model II framework (Argyris \& Schön, 1974). Future studies should investigate the usefulness of this framework for describing and categorizing principal theories of practice and their effectiveness.

Prolonged engagement, triangulation of data, member checks, and other methods supported the trustworthiness of findings in this study, but qualitative research by definition is not generalizable beyond the specific context of study. Future research should explore principal theories of practice in a variety of other contexts, including in high schools (which were not included in the sample for this study). Studies should investigate whether patterns exist in the principal theories of practice in a wide range of 
successful schools, whether differences exist in the principal theories of practice in underperforming or historically unsuccessful schools, and especially which instructional leadership assumptions contribute to the most effective principal theories of practice. Researchers should explore the theory of practice framework of other educational professionals such as teachers and superintendents, and apply the framework to other dimensions of problem solving besides instruction. Other dimensions might include school-community relations, personnel management and recruitment, or managing student discipline.

Ultimately, Argyris and Schön (1974) developed the theory of practice framework not simply as a method for understanding organizational learning, but as a means of enhancing professional effectiveness. Future research should also include quasiexperimental designs in which principals learn about theories of practice, uncover their own theories-in-use, engage in intentional self-reflection and experimentation with revised assumptions and action strategies of instructional leadership, and assess the results (see Polite, 2000). Both quantitative and qualitative methods can contribute to such studies, shedding new light on the cognitive processes involved in reflective practice and the specific assumptions and action strategies that contribute to positive effects on teachers and improvements in student achievement. This study may be a useful starting point for these future research efforts. 


\section{REFERENCES}

Allison, D. J., \& Allison, P. A. (1993). Both ends of a telescope: Experience and expertise in principal problem solving. Educational Administration Quarterly, 29, 302-322.

Anderson, K. D. (2004). The nature of teacher leadership in schools as reciprocal influences between teacher leaders and principals. School Effectiveness and School Improvement, 15, 97-113.

Andrews, R. L., \& Soder, R. (1987, March). Principal leadership and student achievement. Educational Leadership, 44(6), 9-11.

Argyris, C. (1982). Reasoning, learning and action: Individual and organizational. San Francisco: Jossey-Bass.

Argyris, C. (1993). Knowledge for action: A guide to overcoming barriers to organizational change. San Francisco: Jossey-Bass.

Argyris, C., Putnam, R., \& McLain Smith, D (1985). Action Science, Concepts, methods, and skills for research and intervention. San Francisco: Jossey-Bass.

Argyris, C., \& Schön, D. (1974). Theory in practice: Increasing professional effectiveness. San Francisco: Jossey-Bass.

Argyris, C., \& Schön, D. (1978). Organizational learning: A theory of action perspective. Reading, Mass: Addison Wesley.

Basom, M. R., \& Frase, L. (2004). Creating optimal work environments: exploring teacher flow experiences. Mentoring and Tutoring, 12, 241-258.

Barnett, K., \& McCormick, J. (2004). Leadership and individual principal-teacher relationships in schools. Educational Administration Quarterly, 40, 406-434.

Bassett, P. (1998, Fall). Effecting change. Washington, DC: National Association of Independent Schools.

Björk, L. G., \& Keedy, J. L. (2002). Decentralization and school council empowerment in Kentucky: Implications for community relations. Journal of School Public Relations, 23, 30-31. 
Blank, R. K. (1987). The role of principal as leader: Analysis of variation in leadership of urban high schools. Journal of Educational Research, 91, 69-80.

Blase, J., \& Blase, J. R. (1994). Empowering teachers: What successful principals do. Thousand Oaks, CA: Sage.

Blase, J., \& Blase, J. (1997). The fire is back: Principals sharing school governance. Thousand Oaks, CA: Corwin Press.

Blase, J. \& Blase, J. (1998). The handbook of instructional leadership: How really good principals promote teaching and learning. Thousand Oaks, CA: Corwin Press.

Blase, J., \& Blase, J. (1999). Principals' instructional leadership and teacher development: Teachers' perspectives. Educational Administration Quarterly, 35, 349-378.

Blase, J., \& Kirby, P. C. (2000). Bringing out the best in teachers: What effective principals do. Thousand Oaks, CA: Sage.

Blase, J., Blase, J., Anderson, G. L., \& Dungan, S. (1995). Democratic principals in action: Eight pioneers. Thousand Oaks, CA: Sage.

Bossert, S. T., Dwyer, D. C., Rowan, B., \& Lee, G. V. (1982). The instructional management role of the principal. Educational Administration Quarterly, 18(3), 34-64.

Bracey, G. W. (2003). April foolishness: The $20^{\text {th }}$ anniversary of A Nation at Risk. Phi Delta Kappan, 84, 1-8.

Creighton, T. B., \& Jones, G. D. (2001). Selection or self-selection? How rigorous are our selection criteria for education administration preparation programs? Paper presented at the annual meeting of the National Council of Professors of Educational Administration, Houston, TX.

Cuban, L. (1984). Transforming the frog into the prince: Effective schools research, policy and practice at the district level. Harvard Educational Review, 54, 132.

Cusick, P. A. (1992). The educational system: Its nature and logic. New York: McGrallHill, Inc.

Datnow, A. (2005). The sustainability of school reform models in changing district and state contexts. Educational Administration Quarterly, 41, 121-153.

Davis, J., \& Wilson, S. M. (2000). Principal's efforts to empower teachers: Effects on teacher motivation and job satisfaction and stress. Clearing House, 73, 349-353. 
Day, C. (2000). Effective leadership and effective practice. Reflective Practice, 1(1), 113-127.

DeBevoise, W. (1982, Winter). The principal's role: How do we reconcile expectations with reality? $R \& D$ Perspectives.

Din, F. S. (1997, September). The operations of Kentucky's rural school councils. Paper presented at the Annual Meeting of the National Rural Education Association, Tuscon, AZ.

Erlandson, D. (1994). Building a career: Fulfilling the lifetime professional needs of principals. Fairfax, VA: National Policy Board for Educational Administration.

Ferry, N. M., \& Ross-Gordon, J. M. (1998). An inquiry into Schön's epistemology of practice: Exploring links between experience and reflective practice. Adult Education Quarterly, 48 (2), 98-113.

Friedkin, N.E., \& Slater, M. R. (1994). School leadership and performance: A social network approach. Sociology of Education 67, 139-157.

Friedman, V., \& Lipshitz, R. (1992). Teaching people to shift cognitive gears: Overcoming resistance on the road to Model II. Journal of Applied Behavioral Science, 28, 118-136.

Gardner, W. E. (1984). A Nation at risk: Some critical comments. Journal of Teacher Education,35(1), 13-15.

Glaser, B. G., \& Strauss, A. (1967). Discovery of grounded theory: Strategies for qualitative research. Mill Valley, CA: Sociology Press.

Gordon, D. T., Ed. (2003). A nation reformed? American education 20 years after A Nation at Risk. Cambridge, MA: Harvard Education Press.

Guthrie, J. W., \& Springer, M. G. (2004). A nation at risk revisited: Did wrong reasoning result in right results? At what cost?. Peabody Journal of Education, 79, 7-35.

Hallinger, P., \& Heck, R. H. (1998). Exploring the principal's contribution to school effectiveness: 1980 - 1995. School Effectiveness and School Improvement, 9, $157-191$.

Hallinger, P., Bickman, L., \& Davis, K. (1996). School context, principal leadership, and student reading achievement. Elementary School Journal, 96, 527-549.

Hargreaves, A., \& Goodson, I. (2006). Educational change over time? The sustainability and nonsustainability of three decades of secondary school change and continuity. Educational Administration Quarterly, 42, 3-41. 
Heck, R. (1993). School context, principal leadership, and achievement: The case of secondary schools in Singapore. Urban Review, 25, 151-166.

Heck, R. H., Larsen, T. J., \& Marcoulides, G. A. (1990, May). Instructional leadership and school achievement: Validation of a causal model. Educational Administration Quarterly, 26(2), 94-125.

Heck, R. H., \& Marcoulides, G. A. (1990). Examining contextual differences in the development of instructional leadership and school achievement. Urban Review, $22,247-265$.

Holton, G. (2003). An insider's view of A Nation at Risk and why it still matters. Chronicle of Higher Education, 49(33), 1-6.

Howard, J. (2003). "Still at Risk." In Gordon, D.T. (Ed). A nation reformed? American education 20 years after A Nation at Risk. Cambridge, MA: Harvard Education Press.

Hunter, F. (1953). Community power structure. Chapel Hill: University of North Carolina Press.

Keedy, J. L. (2005). "Reconciling the theory and practice schism in education administration through practitioner-developed theories in practice." Journal of Educational Administration,43, 134-153.

Keedy, J. L., \& Achilles, C. M. (1997). The need for school-constructed theories in practice in U.S. school restructuring. Journal of Educational Administration, 35, 102-121.

Keedy, J. L., \& Finch, A. M. (1994). Examining teacher-principal empowerment: An analysis of power. Journal of Research and Development in Education, 27, 154166.

Keedy, J. L., \& Grandy, J. (1999). Trends in GRE scores in education administration: Implications for principal preparation programs. Paper presented at the annual meeting of the American Educational Research Association, Montreal, Quebec. Retrieved May 25, 2006, from EBSCO database.

Keedy, J. L., \& Simpson, D. S. (2001). Principal priorities, school norms, and teacher influence: A study of sociocultural leadership in the high school. Journal of Educational Administration and Foundations, 16, 10-41.

Kentucky Department of Education. (2005). 2005 NCLB interpretive guide: Detailed information about your score reports. Retrieved July 15, 2006, from http://www.education.ky.gov/NR/rdonlyres/ 
ejtpnn12ogdyjom63ciy7bdv3jlyeq7stpljt7tvvbs3xnrqadxhvmkquqpbtebhtvcc3pt5v bsxau43gefr36mb2h/2004NCLBInterpretiveGuideV2\%2e03.doc

Kentucky Department of Education. (n.d.) Public education in Kentucky. Retrieved July 14, 2006, from http://www.education.ky.gov/NR/rdonlyres/ ee2rg5v7trsiexnzfdsmw6f5vki6b737krzhuhvxbe7xp4u6juvkrna3r4u7qh5be4vfssx ilkgwrym5cnkojgrnv2c/publiceducationinkentucky.pdf

Kirby, P. C., \& Paradise, L.V. (1992). Reflective practice and effectiveness of teachers. Psychological Reports, 70, 1057-1058.

Kirby, P.C., \& Teddlie, C. (1989). Development of the Reflective Teaching Instrument. Journal of Research and Development in Education, 22(4), 45-51.

Klecker, B. M., Austin, J. L., \& Burns, L. T. (1999, April). An in-depth analysis of decisions made by Kentucky's school based decision-making councils. Paper presented at the Annual Meeting of the American Educational Research Association, Montreal, Quebec, Canada.

Krug, S. E. (1992). Instructional leadership, school instructional climate, and student outcomes (Project Report). Washington, DC. United States Department of Education. (ERIC Document Reproduction Service No. ED359668)

Leithwood, K., \& Menzies, T. (1998). A review of research concerning the implementation of site-based management. School Effectiveness and Improvement, 9, 233-285.

Leithwood, K., \& Jantzi, D. (1999). Transformational school leadership effects: A replication. School Effectiveness and Improvement, 10, 451-479.

Leithwood, K., Steinbach, R., \& Jantzi, D. (2002). School leadership and teachers' motivation to implement accountability practices. Educational Administration Quarterly, 38, 94-119.

Leithwood, K. A., Steinbach, R., \& Raun, T. (1993). Superintendents' group problemsolving processes. Educational Administration Quarterly, 29, 364-391.

Levine, A. (2005). Educating school leaders. The Education Schools Project. Retrieved April 19, 2008, from www.edschools.org.

Lincoln, Y. S., \& Guba, E. G. (1985). Naturalistic inquiry. Thousand Oaks, CA: Sage.

Marzano, R. J., Pickering, D. J., \& Pollock, J. E. (2001). Classroom instruction that works. Alexandra, VA: ASCD.

Marzano, R. J., Waters, J. T., \& McNulty, B. A. (2005). School leadership that works: 
From research to results. Alexandria, VA: ASCD.

Merriam, S. B. (1997). Qualitative research and case study: Applications in education. San Francisco: Jossey-Bass.

Miles, M. B., \& Huberman, A. M. (1994). Qualitative data analysis: An expanded sourcebook. Thousand Oaks, CA: Sage.

National Center for Educational Statistics (2006, November). NAEP long-term trend data. Retrieved November 19, 2006, from http://nces.ed.gov/nationsreportcard/ltt/.

The National Commission on Excellence in Education. (1983). A nation at risk: The imperative for education reform (GPO Publication No. 065-000-00177-2). Washington, DC: U. S. Government Printing Office.

No Child Left Behind Act of 2001, Public Law 107-110, 115 Stat. 1425

Owens, R. G. (2004). Organizational behavior in education: Adaptive leadership and school reform. Boston: Pearson Publishing.

Pankratz, R. S., \& Petrosko, J. M. (2000). All children can learn: Lessons from the Kentucky reform experience. San Francisco: Jossey-Bass.

Payne, R. K. (2003). A framework for understanding poverty. Highlands, TX: Aha Process.

Petrosko, J. M. (1993, April). The plan for assessing the impact of the Kentucky Education Reform Act (KERA). Paper presented at the annual meeting of the American Educational Research Association. Atlanta, GA.

Polite, V. (2000, February). An effective professional development policy for urban principals: Reflective thinking into practice. Paper presented at the Annual Meeting of the National Association of Secondary School Principals. San Antonio, TX.

Pounder, D. G., Ogawa, R. T., \& Adams, E. A. (1995). Leadership as an organization-wide phenomenon: Its impact on school performance. Educational Administration Quarterly, 31, 564-588.

Rogers, T. (2004). Managing in the interprofessional environment: A theory of action perspective. Journal of Interprofessional Care 18, 239-249.

Rose v. Council for Better Education, 790 S.W.2d186, 60 Ed. Law Rep. 1289 (1989)

Rossman, G. B., \& Rallis, S. F. (1998). Learning in the field: An introduction to 
qualitative research. Thousand Oaks, CA: Sage.

Ruff, W. G., \& Shoho, A. R. (2005). Understanding instructional leadership through the mental models of three elementary principals. Educational Administration Quarterly, 41, 554-577.

Schön, D. A. (1983). The reflective practitioner: How professionals think in action. New York: Basic Books.

Schön, D. (1987). Educating the reflective practitioner. San Francisco: Jossey-Bass.

Senge, P. (1990). The fifth discipline: The art and practice of the learning organization. London: Random House.

Short, P. M. (1994). School empowerment through self-managing teams: Leader behavior in developing self-managing work. Education, 114, 493-593.

Sirotnik, K. A. (1989). The school as a center for change. In T. J. Sergiovanni and J. H. Moore (Eds.). Schooling for tomorrow, pp. 89-113. Allyn and Bacon: Boston, MA.

Snyder, J., \& Ebmeier, H. (1993). Empirical linkages among principal behaviors and intermediate outcomes: Implications for principal evaluation. Peabody Journal of Evaluation, 68, 75-107.

Spillane, J.P., \& Thompson, C. L. (1997). Reconstructing conceptions of local capacity: The local educational agency's capacity for ambitious instructional reform." Educational Evaluation and Policy Analysis, 19, 185-203.

Timperley, H. S. (2005). Distributed leadership: Developing theory from practice. Journal of Curriculum Studies, 37, 395-420.

Tsangaridou, N., \& O'Sullivan, M. (2003). Physical education teachers' theories of action and theories-in-use. Journal of Teaching in Physical Education, 22(2), $132-153$.

Voskuil, S. J. (1999). A Nation at Risk: Commission member's perceptions and appraisals fifteen years later. Unpublished doctoral dissertation. Marquette University.

Wildy, H., \& Dimmock, C. (1993). Instructional leadership in primary and secondary schools in Western Australia. Journal of Educational Administration, 31, 43-31.

Witziers, B., Bosker, R. J., \& Kruger, M. L. (2003). Educational leadership and student achievement: The illusive search for an association. Educational Administration Quarterly, 39, 398-425. 


\title{
GARY W. HOUCHENS
}

\author{
CURRICULUM VITAE
}

\section{EDUCATION}

University of Louisville

Doctor of Philosophy in Educational Leadership, August 2008

- Dissertation: Principal theories of practice: Mapping the cognitive structure and effects of instructional leadership

- Level II Principal certification; Superintendent certification

Oakland City University

Master of Arts in Teaching, June 2003

- Area of Specialization: Education Administration

- Action Research: It's a matter of time: Block scheduling and instructional strategies.

Western Kentucky University

Master of Arts in History, May 2000

- Thesis: A life of paradox: Thomas Merton's Asian trajectory.

Bachelor of Arts in Philosophy and Religion, May 1993

- Minor: History

- Graduated magna cum laude, 3.79 grade point average

\section{PROFESSIONAL EXPERIENCE}

Learning Opportunities Center, Franklin, KY (July 2003 to June 2004; July 2005 to present)

Principal; Juvenile Court Liaison

Lead all aspects of instructional leadership and management of the alternative high school for at-risk students in the Simpson County Schools. Supervise and evaluate teaching and support staff. Supervise finances, scheduling, and professional development. Coordinate implementation of district and school improvement goals. Serve as district liaison to the juvenile court system. Named "Principal of the Year 2007" for work with at-risk youth by the Trabue Group/Family Works Therapy, Bowling Green, KY. 


\section{Interim Principal}

Lead all aspects of management in 850-student high school. Supervised and evaluated more than 90 teachers, support staff and administrators. Supervised curriculum development, personnel, finances and budgeting, scheduling, special education, counseling programs, and all aspects of student supervision. Implemented school improvement plan and coordinated all aspects of strategic planning and professional development.

Mater Dei High School, Evansville, IN (Aug. 2001 to July 2003)

Acting Principal (Oct.2002 to July 2003)

Lead all aspects of management in 600-student high school. Supervised and evaluated more than 60 teachers, support staff, and administrators. Supervised curriculum development, personnel, finances and budgeting, scheduling, special education, counseling programs, and all aspects of student supervision. Served as school liaison to board of education, diocese and all constituency groups, including alumni organizations and area civic groups. Implemented school improvement plan and coordinated all aspects of strategic planning and professional development.

Assistant Principal, Curriculum and Instruction (Aug. 2001 to Oct.2002) Served as instructional supervisor for more than 40 faculty members. Chaired school improvement committee and coordinated all aspects of developing school improvement plan. Coordinated hiring and training of all new staff members. Served as personnel director for all staff, responsible for payroll, benefits management, and staff development. Supervised substitute teachers. Coordinated student scheduling and supervised counseling services. Assisted principal in all aspects of school management.

Christ the King School, Lexington, KY (Aug. 2000 to May 2001) Sixth and Eighth Grade Social Studies and Religion Teacher Served on diocesan social studies curriculum alignment committee, school textbook adoption committee, and all-school liturgy committee. Planned and directed liturgy and worship services for middle school and school-wide celebrations. Volunteer coach with CKS academic team.

Bourbon County Middle School, Paris, KY (Aug. 1998 to May 2000) Sixth Grade Social Studies Teacher and Social Studies Department Chair Served as sixth grade team leader. Trained staff in use of multiple intelligences and learning style theory. Served on school discipline committee and curriculum committee. Sponsored Beta Club and advised peer-mediation program. Collaborated with special education faculty to meet needs of students with learning and behavior disorders. Assistant coach, BCMS football team.

Drakes Creek Middle School, Bowling Green, KY (Aug. 1997 to May 1998) 


\section{Seventh Grade Social Studies Teacher}

Worked with special education teachers to meet the needs of children with learning and behavior disorders. Trained staff in reading comprehension techniques in content areas. Served on district social studies curriculum alignment committee. Coached DCMS's first speech and drama team, including two contestants who competed in the 1998 state tournament.

\section{ACTION RESEARCH}

- Central Office Staffing Structures: A Comparison of High Performing Kentucky Districts. Simpson County Board of Education, 2008.

- It's a Matter of Time: Block Scheduling and Instructional Strategies. Oakland City University, 2003.

\section{CONFERENCE PRESENTATIONS}

- "Mapping the Cognitive Structure and Effects of Instructional Leadership: A Case Study on Theories of Practice," University Council for Educational Administration, Alexandria, VA: November 2007.

- "A Framework for Inquiry: School Change, Student Control, and Theories of Practice," University Council for Education Administration, San Antonio, TX: November 2006.

- "School Change, Student Control, and Theories of Practice: A Conceptual Framework," Spring Student Research Conference, University of Louisville: April 2006.

- "Thomas Merton and the Social Studies Classroom," Kentucky Council for the Social Studies, Lexington, KY: September 2001.

- "Liberal, Conservative, and Beyond: Helping Students Understand the Political Spectrum," Kentucky Council for the Social Studies, Louisville, KY: September 1999.

- "Learning Styles and the Classroom," Kentucky Council for the Social Studies, Lexington, KY: September 1998.

\section{AWARDS \& HONORS}

- Named "2007 Principal of the Year" for work with At-Risk Youth, FamilyWorks Therapy, Bowling Green, KY.

- Named 1998 Best Mannered Teacher, Bowling Green Junior Cotillions.

- Nominated for Sallie Mae First-of-the-Class First Year Teacher Award, 1998.

- Eagle Scout, Boy Scouts of America, December 1986. 
- Simpson County Schools Instructional Leadership Team, ongoing, monthly professional learning community meetings oriented toward continuous professional development and collaborative problem solving.

- "School Law Institute," GRREC, April 2008, Bowling Green, KY.

- University Council for Education Administration conference, November 2007, Alexandria, VA.

- “Thoughtful Classroom” Training, based on research of Silver, Strong, \& Marzano, GRREC, 2004-2007, Bowling Green, KY.

- GGREC Summer Leadership Conference, June 2007, Lexington, KY.

- University Council for Education Administration conference, November 2006, San Antonio, TX.

- CEO Superintendents Network training, "Mike Schmoker: Results Now," October 2006.

- Behavior Institute, Louisville, KY, June 2006.

- University Council for Education Administration conference, November 2005, Nashville, TN.

- Dropout Prevention Seminar, Louisville, KY, May 2004.

- Alternative Schools and Programs Conference, Bowling Green, KY, June 2004.

- Gates Phase II Walkthrough Training, Bowling Green, KY, September 2004.

- College Board, "Administrators: Growing an AP Program," workshop, Lexington, KY, October 2004.

- Notre Dame University “Catholic School Administrator” program, July 2002.

\section{PROFESSIONAL MEMBERSHIPS, ACTIVITIES, \& SERVICE}

- Guest presenter, ELFH 780, Problems in Education Leadership class, Western Kentucky University, February 2008.

- Southern Association of Colleges and Schools (SACS), Accreditation Review Team member, 2007.

- Coordinator of Simpson County Schools Aspiring Administrator Training Program, 2005-2006.

- North Central Association of Colleges and Schools-Commission on Accreditation and School Improvement (NCA-CASI), Accreditation Review Team Member, 2004.

- Member, National Association of Secondary School Principals.

- Member, Kentucky Association of School Administrators.

- Member, Association of Supervision and Curriculum Development.

- Former State Secretary and Executive Committee Member, Kentucky Council for the Social Studies.

- Published poet and author, including publications in Back Home in Kentucky, Frogpond, Modern Haiku and bottle rockets. 